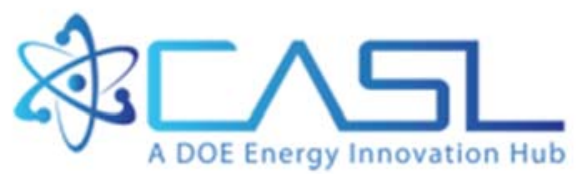

CASL-U-2016-1113-000

\title{
CTF Validation and Verification
}

May 25, 2016
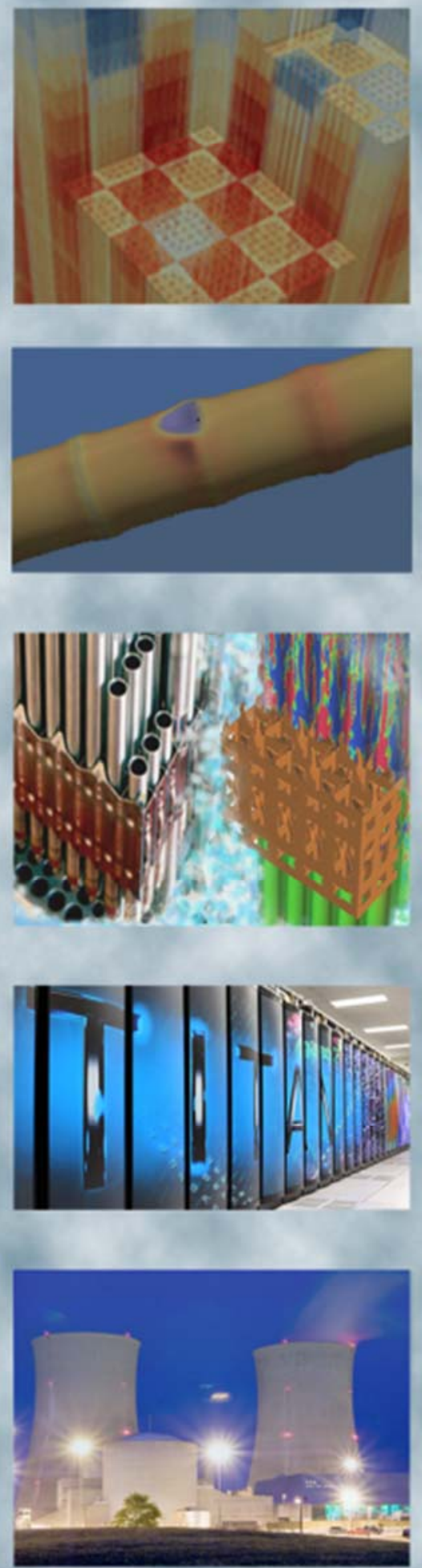


\section{REVISION LOG}

\begin{tabular}{|c|c|c|l|}
\hline Revision & Date & Affected Pages & \multicolumn{1}{c|}{ Revision Description } \\
\hline 0 & & All & Initial Version \\
\hline & & & \\
\hline & & & \\
\hline & & & \\
\hline
\end{tabular}

Document pages that are:

Export Controlled ____ None

IP/Proprietary/NDA Controlled None

Sensitive Controlled____ None

\section{Requested Distribution:}

To: Unlimited distribution

Copy: 


\title{
DOCUMENT AVAILABILITY
}

Reports produced after January 1, 1996, are generally available free via US Department of Energy (DOE) SciTech Connect.

Website http://www.osti.gov/scitech/

Reports produced before January 1, 1996, may be purchased by members of the public from the following source:

\author{
National Technical Information Service \\ 5285 Port Royal Road \\ Springfield, VA 22161 \\ Telephone 703-605-6000 (1-800-553-6847) \\ TDD 703-487-4639 \\ Fax 703-605-6900 \\ E-mail info@ntis.gov \\ Website http://www.ntis.gov/help/ordermethods.aspx
}

Reports are available to DOE employees, DOE contractors, Energy Technology Data Exchange representatives, and International Nuclear Information System representatives from the following source:

Office of Scientific and Technical Information

PO Box 62

Oak Ridge, TN 37831

Telephone 865-576-8401

Fax 865-576-5728

E-mail reports@osti.gov

Website http://www.osti.gov/contact.html

This report was prepared as an account of work sponsored by an agency of the United States Government. Neither the United States Government nor any agency thereof, nor any of their employees, makes any warranty, express or implied, or assumes any legal liability or responsibility for the accuracy, completeness, or usefulness of any information, apparatus, product, or process disclosed, or represents that its use would not infringe privately owned rights. Reference herein to any specific commercial product, process, or service by trade name, trademark, manufacturer, or otherwise, does not necessarily constitute or imply its endorsement, recommendation, or favoring by the United States Government or any agency thereof. The views and opinions of authors expressed herein do not necessarily state or reflect those of the United States Government or any agency thereof. 
The Pennsylvania State University

Department of MechanicAl AND Nuclear EngineERING

Reactor Dynamics and Fuel Management Group

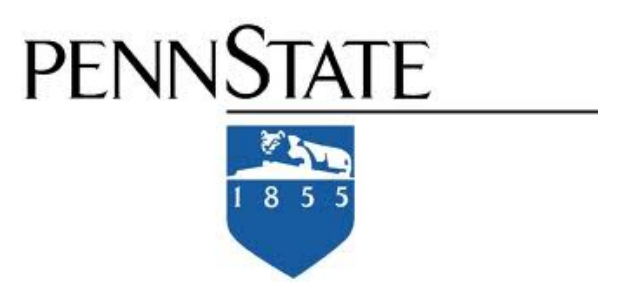

\section{CTF Validation and Verification}

May 25, 2016 


\section{Authors:}

Robert K. Salko ${ }^{1}$

Taylor S. Blyth ${ }^{2}$

Chris A. Dances ${ }^{3}$

Jeffrey W. Magedanz ${ }^{4}$

Caleb Jernigan ${ }^{5}$

Joeseph Kelly ${ }^{6}$

Aysenur Toptan ${ }^{7}$

Marcus Gergar ${ }^{8}$

Chris Gosdin ${ }^{9}$

Maria N. Avramova ${ }^{10}$

Scott Palmtag ${ }^{11}$

Jess C. Gehin ${ }^{12}$

\footnotetext{
${ }^{1}$ Oak Ridge National Laboratory T: (856) 576-5339 E: salkork@ornl.gov

${ }^{2}$ Pennsylvania State University

${ }^{3}$ Pennsylvania State University

${ }^{4}$ Pennsylvania State University

${ }^{5}$ Holtec International

${ }^{6}$ United State Nuclear Regulatory Commission

${ }^{7}$ North Carolina State University

${ }^{8}$ Pennsylvania State University

${ }^{9}$ Pennsylvania State University

${ }^{10}$ Pennsylvania State University

${ }^{11}$ Core Physics

${ }^{12}$ Oak Ridge National Laboratory
} 


\begin{abstract}
Coolant-Boiling in Rod Arrays- Two Fluids (COBRA-TF) is a Thermal/Hydraulic $(\mathrm{T} / \mathrm{H})$ simulation code designed for Light Water Reactor (LWR) analysis. It uses a two-fluid, threefield (i.e. fluid film, fluid drops, and vapor) modeling approach. Both sub-channel and 3D Cartesian forms of nine conservation equations are available for LWR modeling. The code was originally developed by Pacific Northwest Laboratory in 1980 and has been used and modified by several institutions over the last several decades. COBRA-TF is also used at the Pennsylvania State University (PSU) by the Reactor Dynamics and Fuel Management Group (RDFMG) and has been improved, updated, and subsequently became the PSU RDFMG version of COBRATF (CTF). One part of the improvement process includes validating the methods in CTF.

This document seeks to provide a certain level of certainty and confidence in the predictive capabilities of the code for the scenarios it was designed to model - rod bundle geometries with operating conditions that are representative of prototypical Pressurized Water Reactor (PWR)s and Boiling Water Reactor (BWR)s in both normal and accident conditions. This is done by modeling a variety of experiments that simulate these scenarios and then presenting a qualitative and quantitative analysis of the results that demonstrates the accuracy to which CTF is capable of capturing specific quantities of interest.
\end{abstract}


1 Introduction 2

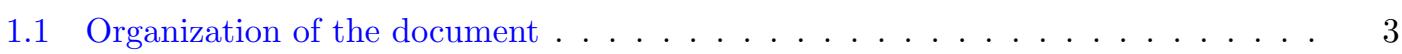

1.2 Comparison Metrics . . . . . . . . . . . . . . . . . . . . 3

2 Validation Plan 5

$\begin{array}{llr}3 & \text { Test Descriptions } & 9\end{array}$

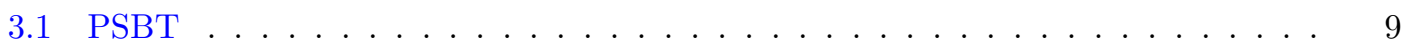

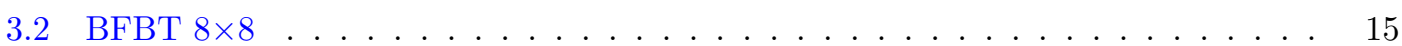

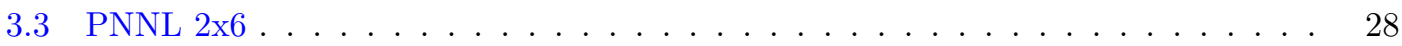

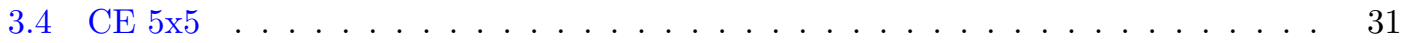

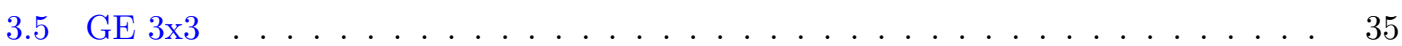

3.6 Harwell High Pressure Loop . . . . . . . . . . . . . . . . . . . . . . . . . 39

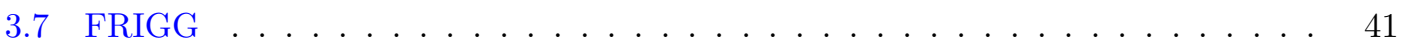

3.7.1 CTF Model of Facility . . . . . . . . . . . . . . . . . . . . . . . . . . 41

3.7.1.1 Radial Meshing . . . . . . . . . . . . . . . . . . 41

3.7.1.2 Axial Meshing . . . . . . . . . . . . . . . 46

3.7.1.3 Boundary Conditions . . . . . . . . . . . . . . . . . 46 
3.7.1.4 Modeling Choices ................... 46

3.8 RPI $2 \times 2 \ldots \ldots \ldots \ldots \ldots \ldots \ldots$

3.9 Kumamoto Univeristy $2 \times 3$. . . . . . . . . . . . . . . . . 52

4 Heat Transfer $\quad 56$

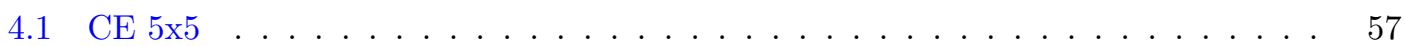

5 Pressure loss $\quad 63$

5.1 Pressure drop in $\mathrm{CTF} \ldots \ldots \ldots \ldots \ldots$. . . . . . . . . . . . . . . . 63

5.2 Single-phase pressure drop . . . . . . . . . . . . . . . . . . . 64

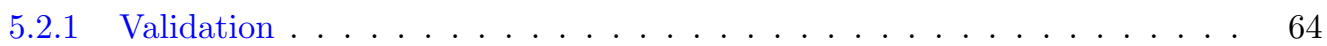

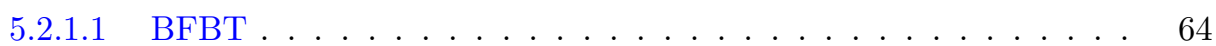

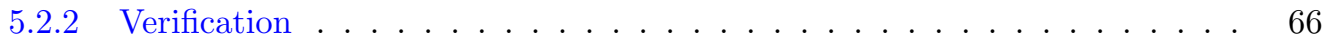

5.2.2.1 Single-Phase Friction Flow Split . . . . . . . . . . . . 66

5.2 .2 .2 Friction Model Verification . . . . . . . . . . . . 70

5.3 Two-phase pressure drop . . . . . . . . . . . . . . . . . 78

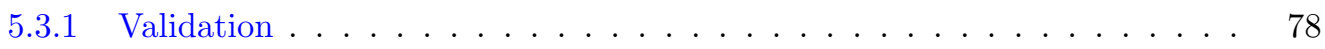

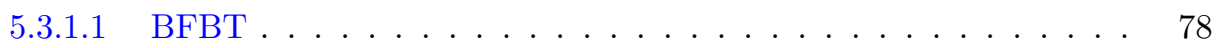

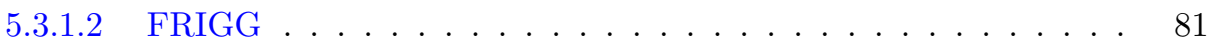

6 Single-Phase Turbulent Mixing $\quad 83$

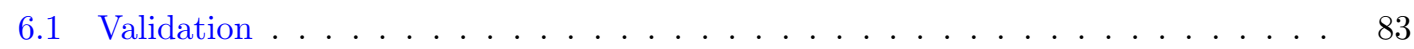

$6.1 .1 \mathrm{CE} 5 \times 5 \ldots \ldots \ldots \ldots$

6.1 .2 Kumamoto University $2 \times 3 \ldots \ldots$. . . . . . . . . . . 88

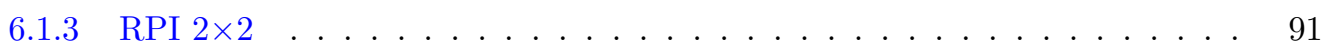

6.1 .4 GE $3 \times 3 \ldots \ldots \ldots \ldots 7$

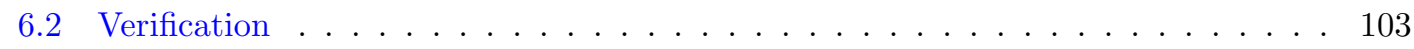

6.2.1 Single-Phase Two-Channel .................... 103 
7 Void Content $r$

$7.1 \mathrm{PSBT} \ldots \ldots \ldots \ldots \ldots \ldots \ldots$

7.2 FRIGG Facility . . . . . . . . . . . . . . . . . . . 113

8 Two-Phase Turbulent Mixing and Void Drift $\quad 115$

8.1 Validation . . . . . . . . . . . . . . . . . . . 115

$8.1 .1 \mathrm{GE} 3 \mathrm{x} 3 \ldots \ldots \ldots \ldots \ldots \ldots$

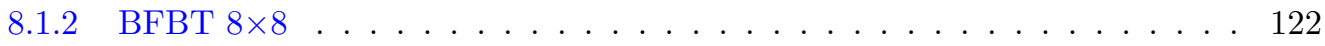

9 Dryout $\quad 150$

9.1 Validation ............................ 150

9.1 .1 Harwell Facility . . . . . . . . . . . . . . . . . . . . 150

9.1 .2 Takahama Tests . . . . . . . . . . . . . . . . 154

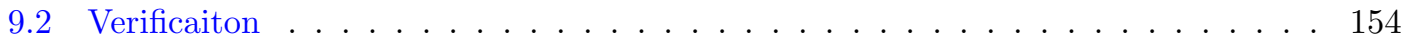

9.2.1 Bowring Correlation ..................... 154

9.2.2 Groeneveld Look-up Table . . . . . . . . . . . . . . . . 155

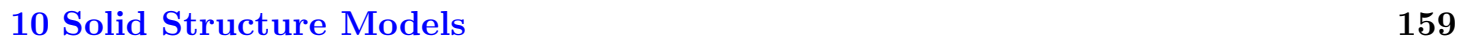

10.1 Inside Tube Flow . . . . . . . . . . . . . . . . . . . . . . . . . 159

10.1 .1 Direct Heat Input . . . . . . . . . . . . . . . . . . 160

10.1.2 Radial Conduction Test . . . . . . . . . . . . . . . . . 160

10.1 .3 Multiple Inside Channels . . . . . . . . . . . . . . . . . . . 162

11 Natural Circulation $\quad 164$

11.1 PNNL $2 \mathrm{x} 6$ Tests $\ldots \ldots \ldots \ldots \ldots \ldots$

12 Conclusion $\quad 175$

$\begin{array}{lr}\text { Bibliography } & 175\end{array}$

$\begin{array}{lr}\text { A Nomenclature } & 178\end{array}$ 
3.1 Summary of PSBT rod-bundle configurations for CTF validation activities . . . . 10

3.2 Assembly $0-1,0-2$, and $0-3$ description $\ldots \ldots \ldots \ldots$. . . . . . . . . 17

3.3 Assembly 0-1, 0-2, and 0-3 description (continued) . . . . . . . . . . . 18

3.4 Assembly 1, 2, and 3 description . . . . . . . . . . . . . . . . . 19

3.5 Assembly 1 power . . . . . . . . . . . . . . . . . . . . . . 19

3.6 Assembly 4 power . . . . . . . . . . . . . . . . . . . 20

3.7 Assembly 4 description . . . . . . . . . . . . . . . . . . . . 20

3.8 BFBT bundle pressure tap locations . . . . . . . . . . . . . . . . 21

3.9 BFBT void measurements . . . . . . . . . . . . . . . . . . 23

3.10 Assembly $0-1,0-2,0-3$, and 1 flow areas . . . . . . . . . . . 25

3.11 Assembly 4 flow areas . . . . . . . . . . . . . . . . . . . 25

$3.12 \mathrm{C} 2 \mathrm{~A}$ ferrule grid geometry . . . . . . . . . . . . . . . . 26

3.13 BFBT loss coefficients . . . . . . . . . . . . . . . . . . 27

3.14 Cross-sectional geometry of the PNNL 2 x6 test section . . . . . . . . . . . . 29

3.15 Axial schematic of the PNNL 2x6 facility and CTF meshing (units shown in inches) 29

3.16 Cross-section diagram of CE $5 \times 5$ Test $74 \ldots \ldots \ldots$. . . . . . . . . . 32 
3.17 Cross-section diagram of CE $5 \times 5$ Test $75 \ldots \ldots \ldots \ldots$

3.18 Axial schematic of the CE $5 \times 5$ bundle . . . . . . . . . . . . . . . . . . 34

3.19 GE $3 \times 3$ Bundle Geometry . . . . . . . . . . . . . . . . . . . . 36

3.20 GE $3 \times 3$ Bundle Pin Spacer Geometry . . . . . . . . . . . . . . . . . 38

3.21 CTF model of the Harwell High-Pressure Two-Phase Test Facility . . . . . . . 40

3.22 Cross-section of the FRIGG facility (including labels of CTF model rods and

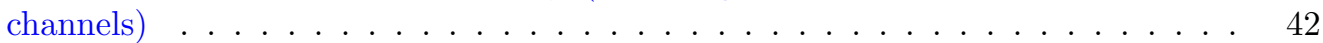

3.23 Definition of functions for the circle that defines the fuel rod and the circle it sits on 43

3.24 Schematic of FRIGG facility in axial direction . . . . . . . . . . . . . . . . 47

3.25 Low Assembly Test Section with Sinter Section Shown [14] . . . . . . . . . . . . 49

3.26 Air/Water Mixing Tee $[14] \ldots \ldots \ldots \ldots \ldots$

3.27 NUREG/CR-3373 Experimental Cross Section [14] . . . . . . . . . . . . 51

$3.282 \times 3$ facility cross section . . . . . . . . . . . . . . . . . 53

$3.292 \times 3$ facility side view . . . . . . . . . . . . . . . . . . . . . 54

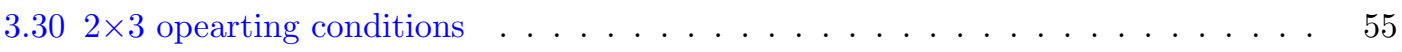

4.1 Rod surface measurements and predictions with respect to increasing test heat flux for Rod 25 (central rod) at 77.63 inch axial location . . . . . . . . . . . . 58

4.2 Summary of mean difference between predicted and measured rod surface temperatures for each thermocouple in Rod 25 over all test cases . . . . . . . . . . 59

4.3 CE 5x5 Test 74 Mixing Vane Grid . . . . . . . . . . . . . . . . . . 61

4.4 Summary of mean difference between predicted and measured rod surface temperatures for each thermocouple in Rod 24 over all test cases . . . . . . . . . . .

5.1 BFBT single-phase pressure drop predictions (Series 7) . . . . . . . . . . 65

5.2 Diagram of the two-channel flow split problem. . . . . . . . . . . . . . . 69

5.3 Two-channel flow-split results . . . . . . . . . . . . . . . . . . . 70

$5.43 \times 3$ rod bundle geometry with (a) rod-centered and (b) channel-centered sub-

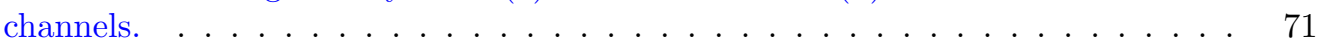

5.5 Ratio of measured-to-predicted pressure drop compared to bundle-average exit quality for BFBT two-phase tests (Series P6) . . . . . . . . . . . 
5.6 Comparison of measured and predicted bundle-averaged exit quality for BFBT P6

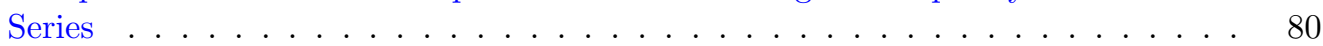

5.7 Comparison of experimental and predicted components of pressure drop . . . . .

6.1 Average difference between CTF predicted channel exit temperatures and experimental values for all tests in CE $5 \times 5$ Series $74 \ldots \ldots$. . . . . . . . . . . .

6.2 Average difference between CTF predicted channel exit temperatures and experimental values with measurement error and mean discrepancy

6.3 Predicted and measured outlet temperatures for Channel 36 in all CE $5 \times 5$ Series 74 cases ............................... 87

$6.42 \times 3$ results using Rogers and Rosehart . . . . . . . . . . . . . . . . . . 89

$6.52 \times 3$ results using $\beta=0.004 \ldots \ldots \ldots \ldots \ldots$

$6.62 \times 3$ results using $\beta=0.007 \ldots \ldots \ldots \ldots \ldots$

$6.72 \times 3$ results using CTF friction model f . . . . . . . . . . . . . . . 90

6.8 Watts Bar simulation sensitivity to $\beta \ldots \ldots \ldots \ldots 1$

$6.92 \times 2$ Case 1 results with no mixing . . . . . . . . . . . . . . 93

$6.102 \times 2$ Case 2 results with no mixing . . . . . . . . . . . . . . 93

$6.112 \times 2$ Case 1 results with mixing . . . . . . . . . . . . . . . 95

$6.122 \times 2$ Case 2 results with mixing . . . . . . . . . . . . . . . 95

$6.132 \times 2$ Case 1 results with $\beta$ perturbation . . . . . . . . . . . . . 96

$6.142 \times 2$ Case 2 results with $\beta$ perturbation $\ldots \ldots \ldots . \ldots \ldots$

6.15 GE $3 \times 3$ Case 1 B results with no mixing . . . . . . . . . . . . . . . 98

6.16 GE $3 \times 3$ Case $1 \mathrm{C}$ results with no mixing . . . . . . . . . . . . . . 98

6.17 GE $3 \times 3$ Case 1 D results with no mixing . . . . . . . . . . . . . . . 99

6.18 GE $3 \times 3$ Case 1 E results with no mixing . . . . . . . . . . . . . . . 99

6.19 GE $3 \times 3$ Case 1 B results with mixing . . . . . . . . . . . . . . . . 100

6.20 GE $3 \times 3$ Case $1 \mathrm{C}$ results with mixing . . . . . . . . . . . . . . . . 100

6.21 GE $3 \times 3$ Case $1 \mathrm{D}$ results with mixing . . . . . . . . . . . . . . . . . 101

6.22 GE $3 \times 3$ Case 1 E results with mixing . . . . . . . . . . . . . . . . . 101 
6.23 GE $3 \times 3$ single-phase test summary . . . . . . . . . . . . . . . . . . 102

6.24 GE $3 \times 3$ single-phase test summary using Rogers and Rosehart . . . . . . . . . . 102

6.25 Model of problem for testing single-phase turbulent mixing of enthalpy. . . . . . 104

6.26 Turbulent-mixing problem results . . . . . . . . . . . . . . . . . 107

6.27 Mixing problem mass flow rates . . . . . . . . . . . . . . 107

6.28 Mixing problem mass flow rates with no mixing . . . . . . . . . . . . 108

7.1 PSBT Series 5 predicted versus measured void around central rod in bundle . . . 110

7.2 PSBT Series 6 predicted versus measured void around central rod in bundle . . . 111

7.3 PSBT Series 7 predicted versus measured void around central rod in bundle . . . 112

7.4 Predicted and measured axial bundle-averaged void profile in FRIGG facility . . 114

8.1 GE $3 \times 3$ quality summary . . . . . . . . . . . . . . . . 117

8.2 GE $3 \times 3$ quality summary without void drift . . . . . . . . . . . . . 117

8.3 GE $3 \times 3$ mass flux summary . . . . . . . . . . . . . . . . . . . 118

8.4 GE $3 \times 3$ mass flux summary without void drift . . . . . . . . . . . . . 118

8.5 GE $3 \times 3$ mass flux summary using Rogers and Rosehart . . . . . . . . . . . . . . 119

8.6 GE $3 \times 3$ quality summary using Rogers and Rosehart . . . . . . . . . . . . . . . 119

8.7 GE $3 \times 3$ Case $2 \mathrm{C} 1$ flow split . . . . . . . . . . . . . . . . . 120

8.8 GE $3 \times 3$ Case $2 \mathrm{C} 2$ flow split . . . . . . . . . . . . . . . . . . 121

8.9 GE $3 \times 3$ Case $2 \mathrm{G} 1$ flow split . . . . . . . . . . . . . . . . . . . . . . . 121

8.10 GE $3 \times 3$ Case 2 D3 flow split . . . . . . . . . . . . . . . . . 122

8.11 Comparison of measured and predicted bundle-averaged outlet void. . . . . . . . 123

8.12 Comparison of measured and predicted bundle-averaged outlet thermal equilibrium quality. . . . . . . . . . . . . . . . . . . . 124

8.13 Assembly $0-1$ void . . . . . . . . . . . . . . . . . . 125

8.14 Assembly $0-2$ void . . . . . . . . . . . . . . . . . . . 126

8.15 Assembly $0-3$ void . . . . . . . . . . . . . . . . . . . . 127 
8.16 Assembly 1 void . . . . . . . . . . . . . . . . . . . . . . 127

8.17 Assembly 4 void . . . . . . . . . . . . . . . . . 128

8.18 BFBT void summary . . . . . . . . . . . . . . . . . . . . . 129

8.19 BFBT void summary with droplets . . . . . . . . . . . . . . . . . . . . . 129

8.20 BFBT corner channel void . . . . . . . . . . . . . . . . . . . . 130

8.21 BFBT corner channel void with droplets . . . . . . . . . . . . . . . . 130

8.22 BFBT side channel void . . . . . . . . . . . . . . . . . . . . . . . . . 131

8.23 BFBT side channel void with droplets . . . . . . . . . . . . . . . 131

8.24 BFBT inner channel void . . . . . . . . . . . . . . . . . . . 132

8.25 BFBT inner channel void with droplets . . . . . . . . . . . . . . . 132

8.26 BFBT unheated channel void . . . . . . . . . . . . . . . . 133

8.27 BFBT unheated channel void with droplets . . . . . . . . . . . . . 133

8.28 BFBT void with no void drift . . . . . . . . . . . . . . . . 134

8.29 BFBT corner void with no void drift . . . . . . . . . . . . . . . . 134

8.30 BFBT side void with no void drift $\ldots \ldots \ldots \ldots \ldots \ldots \ldots$

8.31 BFBT inner void with no void drift . . . . . . . . . . . . . 135

8.32 BFBT unheated void with no void drift $\ldots \ldots \ldots \ldots \ldots$

8.33 Example of diagonal lines from where subchannel void data is extracted. . . . . . 137

8.34 Measured and predicted void along diagonals of Test 0011-55 . . . . . . . . . 138

8.35 Measured and predicted void along diagonals of Test 0011-55 with void drift disabled.138

8.36 Measured and predicted void along diagonals of Test 0011-61 . . . . . . . . . . 140

8.37 Measured and predicted void along diagonals of Test 0011-61 with void drift disabled.140 8.38 Measured and predicted void along diagonals of Test 0021-16 . . . . . . . . . . 141

8.39 Measured and predicted void along diagonals of Test 0021-16 with void drift disabled.141

8.40 Measured and predicted void along diagonals of Test 0021-21 . . . . . . . . . . . 142

8.41 Measured and predicted void along diagonals of Test 0021-21 with void drift disabled.142

8.42 Measured and predicted void along diagonals of Test 0031-16 . . . . . . . . . . 143 
8.43 Measured and predicted void along diagonals of Test 0031-16 with void drift disabled.143

8.44 Measured and predicted void along diagonals of Test 0031-21. . . . . . . . . . 144

8.45 Measured and predicted void along diagonals of Test 0031-21 with void drift disabled.144

8.46 Measured and predicted void along diagonals of Test 1071-55. . . . . . . . . . . . 146

8.47 Measured and predicted void along diagonals of Test 1071-55 with void drift disabled.146

8.48 Measured and predicted void along diagonals of Test 1071-61. . . . . . . . . . . . 147

8.49 Measured and predicted void along diagonals of Test 1071-61 with void drift disabled.147

8.50 Measured and predicted void along diagonals of Test 4101-53. . . . . . . . . . . . 148

8.51 Measured and predicted void along diagonals of Test 4101-53 with void drift disabled.148

8.52 Measured and predicted void along diagonals of Test 4101-61. . . . . . . . . . . . 149

8.53 Measured and predicted void along diagonals of Test 4101-61 with void drift disabled.149

9.1 Comparison of predicted and experimental axial temperature profiles for Harwell Test $5358 \ldots \ldots \ldots \ldots \ldots \ldots \ldots \ldots \ldots$

9.2 Summary of predicted and experimental dryout locations for Harwell test cases modeled by $\mathrm{CTF} \ldots \ldots \ldots \ldots \ldots$. . . . . . . . . . . . . . . . . . . . . . . . . .

9.3 Groeneveld Hand Interpolation Scheme . . . . . . . . . . . . . . . . . 157

10.1 CTF predicted wall temperature drop compared to analytical solution for channel on outside versus channel on inside . . . . . . . . . . . . . . . . 162

11.1 Predicted and measured subchannel velocities for Window 1 at Rake Location $\mathrm{Y}=-0.581$ in in PNNL $2 \mathrm{x} 6 \ldots \ldots \ldots \ldots$

11.2 Predicted and measured subchannel velocities for Window 3 at Rake Location $\mathrm{Y}=-0.581$ in in PNNL $2 \mathrm{x} 6 \ldots \ldots \ldots \ldots 6$

11.3 Predicted and measured subchannel velocities for Window 5 at Rake Location $\mathrm{Y}=-0.581$ in in PNNL $2 \mathrm{x} 6 \ldots \ldots \ldots$. . . . . . . . . . . . . . . . . . . . . . . .

11.4 Predicted and measured subchannel velocities for Window 1 at Rake Location $\mathrm{Y}=0.0$ in in PNNL $2 \mathrm{x} 6 \ldots \ldots \ldots \ldots \ldots . \ldots$

11.5 Predicted and measured subchannel velocities for Window 3 at Rake Location $\mathrm{Y}=0.0$ in in PNNL $2 \mathrm{x} 6 \ldots \ldots \ldots \ldots \ldots$

11.6 Predicted and measured subchannel velocities for Window 5 at Rake Location $\mathrm{Y}=0.0$ in in PNNL $2 \mathrm{x} 6 \ldots \ldots \ldots \ldots$. . . . . . . . . . . . . . . . . . . . . 
11.7 Predicted and measured subchannel velocities for Window 7 at Rake Location $\mathrm{Y}=0.0$ in in PNNL $2 \mathrm{x} 6 \ldots \ldots \ldots \ldots$

11.8 Predicted and measured subchannel velocities for Window 1 at Rake Location $\mathrm{Y}=0.581$ in in PNNL $2 \mathrm{x} 6 \ldots \ldots \ldots \ldots$. . . . . . . . . . . . . . . . . . .

11.9 Predicted and measured subchannel velocities for Window 3 at Rake Location $\mathrm{Y}=0.581$ in in PNNL $2 \mathrm{x} 6 \ldots \ldots \ldots \ldots$

11.10Predicted and measured subchannel velocities for Window 5 at Rake Location $\mathrm{Y}=0.581$ in in PNNL $2 \mathrm{x} 6 \ldots \ldots \ldots$. . . . . . . . . . . . . . 170

11.11Predicted and measured subchannel velocities for Window 7 at Rake Location $\mathrm{Y}=0.581$ in in PNNL $2 \mathrm{x} 6 \ldots \ldots \ldots \ldots \ldots$

11.12Predicted and measured subchannel-center temperatures for Window 1 in PNNL $2 \mathrm{x} 6 \ldots \ldots \ldots \ldots \ldots \ldots \ldots \ldots$

11.13Predicted and measured subchannel-center temperatures for Window 3 in PNNL

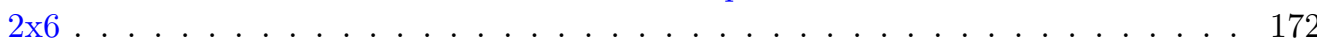

11.14Predicted and measured subchannel-center temperatures for Window 5 in PNNL $2 \times 6 \ldots \ldots \ldots \ldots \ldots \ldots$

11.15Predicted and measured subchannel-center temperatures for Window 7 in PNNL

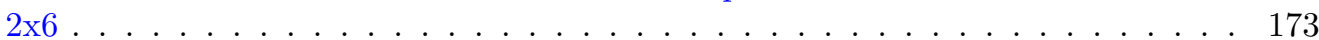

11.16Predicted and measured subchannel-center temperatures for Window 9 in PNNL $2 \times 6 \ldots \ldots \ldots \ldots \ldots \ldots \ldots$ 


\title{
CHAPTER 1
}

INTRODUCTION

\begin{abstract}
Modeling and simulating a physical system or phenomena is a complex process involving several steps. We must formulate a mathematical model that suitably describes the physics we look to model, we need to come up with a way to solve that mathematical model, and finally, we need to then translate all these steps into machine language in the form of a computer program to make the method useful for solving real problems. Additionally, each of these steps - the model selection, assumptions, and coding - must be thoroughly tested and documented so as to instill confidence in the user that the resulting product is useful for its intended purpose. Ideally, good code documentation should inform the user:
\end{abstract}

1. what the code does,

2. how the code works,

3. how to use the code, and

4. that the code works as advertised.

The CTF documentation suite includes both a User Manual[1] and a Theory Manual[2], which address Item 3 (how to use the code) and Item 2 (how the code works). This document addresses Item 1 (what the code does) and Item 4 (that the code works as advertised). Note that Item 4 covers code testing, which is a broad topic that includes both verification and validation tasks. Whereas verification tasks demonstrate that the mathematical equations are being solved correctly and were implemented into the code in a bug-free way, validation tasks demonstrate that the correct equations are being solved, resulting in good agreement with experimental data. Comparing to experimental data is a necessary part of validation work. This document covers both validation and verification testing that is performed for CTF.

An important aspect of this Validation \& Verification $(V \& V)$ suite is its tight integration in the CASL version of CTF. All of the tests created as part of this study have been included 
directly in the CTF code repository. Additionally, many of the tests are used as code regression tests that are run on a regular basis in an automated fashion. Because the testing process is automated, it is possible for the user to re-run the validation suite at the "push of a button". This instills a higher level of confidence in the modeling and simulation capabilities of CTF and helps to keep this validation study relevant, despite ongoing development activities in the code.

Note that while many of these tests have been incorporated as regression tests (meaning tests that are run on a continual basis to demonstrate code results are not changing), this document does not serve as a single, comprehensive reference for all regression testing performed on CTF. Many additional regression tests are included in the CTF automated test matrix that do not have experimental results or an analytical solution to compare against; rather, they simply exercise some feature or combination of features that have an effect on the output file, which is checked against a gold version. Documentation for such tests can be found directly in the "CMakeLists.txt" file that drives the automated test matrix (i.e., COBRA-TF/cobra_tf/test_matrix/CMakeLists.txt) or in the corresponding test input files found in COBRA-TF/cobra_tf/test_matrix.

\subsection{Organization of the document}

Chapter 2 gives the plan that is used to drive CTF V\&V efforts. This document does not seek to "validate CTF"; rather, it attempts to "validate CTF for specific applications". This chapter will outline the intended applications of CTF, discuss the physical phenomena important to these applications, the CTF models used to capture these phenomena, and present the tests used to ensure the correct functioning of these models.

Chapter 3 gives an overview of the experiments that were used for the validation study. Emphasis is placed on a brief overview of the design of the tests and their purpose as well as how CTF was used to model the tests.

The remaining chapters cover the $\mathrm{V} \& \mathrm{~V}$ efforts for each of the intended code applications discussed in Chapter 2.

\subsection{Comparison Metrics}

In the following analysis, it is necessary to choose some metrics for comparing the accuracy of $\mathrm{CTF}$ in predicting the numerous Quantity of Interest (QOI)s. Most of this analysis is rather standard and simple, but for the sake of being clear, some definitions are provided here.

We employ simple averaging to calculate average predicted and measured absolute values and, at times, also the average difference between measured and predicted values. This will be referred to as the "mean" or referenced symbolically as $\mu$ and is calculated as in Equation 1.1. In the equation, $N$ is the number of values in the sample and $x_{i}$ is the sampled value.

$$
\mu=\Sigma_{i=1}^{N}\left(\frac{x_{i}}{N}\right)
$$


The standard deviation, is in reference to the spread of the data that was averaged using Equation 1.1. It is calculated as shown in Equation 1.2.

$$
\sigma=\sqrt{\sum_{i=1}^{N}\left(\frac{\left(x_{i}-\mu\right)^{2}}{N}\right)}
$$

In order to make direct comparisons between the measured and predicted values, the Rootmean-square error (RMSE) is used, which is shown in Equation 1.3. It is a common metric for comparing predicted and measured values and is similar to the standard deviation, except that it compares the distances of the predictions from measured values. In the equation, $x_{\mathrm{ctf}}$ is the predicted value and $x_{\mathrm{m}}$ is the corresponding measured value. A note about this formulation is that it will exaggerate large differences between predictions and measured data due to the squaring of the difference. Outliers are removed where appropriate, but this is clearly noted in the text when done.

$$
\mathrm{RMSE}=\sqrt{\frac{1}{N} \sum_{i=1}^{N}\left(x_{\mathrm{ctf}, i}-x_{\mathrm{m}, i}\right)^{2}}
$$

Both the standard deviation and RMSE will retain the units of the sampled value. This can be problematic when we want to compare data sets that were sampled from different operating conditions; in such a case, we expect the magnitude of the differences between measured and predicted results to change, so what we really want is to quantify the average of the relative differences. This is accomplished by taking the relative root-mean-square (rRMS) of the relative errors between measured and computed data. The relative error is defined as shown in Equation 1.4. The rRMS of the relative errors is defined as shown in Equation 1.5.

$$
\begin{gathered}
E_{\text {rel }}=\frac{x_{\text {measured }}-x_{\text {predicted }}}{x_{\text {measured }}} \\
\operatorname{rRMS}=\sqrt{\frac{1}{N} \sum_{i=1}^{N} E_{\text {rel }, i}^{2}}
\end{gathered}
$$




\section{CHAPTER 2}

\section{VALIDATION PLAN}

CTF is being developed in Consortium for Advanced Simulation of Light Water Reactors (CASL) for modeling specific applications. The validation and verification work is meant to demonstrate that the code behaves as expected and produces physically correct and accurate results for these intended applications. Therefore, the intended applications should drive the validation and verification work. This section specifies the intended modeling applications of CTF. Each application has a set of physical phenomena upon which it will be dependent. CTF must be capable of accurately modeling each of these physical phenomena. A series of tables follow that summarize the list of intended applications, the important physical phenomena in these applications, the models that were implemented into CTF to capture these important physical phenomena, and a link to the section that documents the tests that were developed to show how accurately these models capture the phenomena. There are two columns for tests; one provides a link to validation tests and the other provides links to verification tests. A separate table is provided for each intended application of the code. CTF is being developed for the following modeling applications:

1. Normal PWR operating behavior (Table 2.1)

2. Normal BWR operating behavior (Table 2.2)

3. Departure from Nucleate Boiling (DNB) analysis of PWRs (Table 2.4)

4. Crud-induced power shift (CIPS) analysis of PWRs (Table 2.3)

5. Reactivity-Insertion Accident (RIA) analysis in PWRs (Table 2.5)

A blank spot in the "Validation" or "Verification" column of these tables means that that particular model has not yet been tested. The reader should be aware that this is a living document and that these tables are meant to define a roadmap that will be used to develop and test CTF for its intended applications. Blank spots in the test matrix will be addressed in future revisions. 
Table 2.1: Validation requirements and activities for modeling of steady-state, normal PWR operations

\begin{tabular}{|c|c|c|c|}
\hline Phenomena & Model & $\begin{array}{r}\text { Validation } \\
\text { Tests }\end{array}$ & $\begin{array}{r}\text { Verification } \\
\text { Tests }\end{array}$ \\
\hline Single-phase convection & Dittus-Boelter & 4 & - \\
\hline $\begin{array}{r}\text { Subcooled-boiling heat } \\
\text { transfer }\end{array}$ & Thom & 4 & - \\
\hline $\begin{array}{r}\text { Single-phase grid spacer } \\
\text { pressure loss }\end{array}$ & Form loss & 5.2 .1 & - \\
\hline Single-phase wall shear & Darcy-Weisbach & 5.2 .1 & 5.2 .2 \\
\hline $\begin{array}{r}\text { Grid heat transfer } \\
\text { enhancement }\end{array}$ & Yao-Hochreiter-Leech & - & - \\
\hline $\begin{array}{r}\text { Single-phase Turbulent } \\
\text { mixing }\end{array}$ & Mixing-length theory & 6.1 & 6.2 \\
\hline Pressure-directed cross flow & Transverse momentum equation & - & - \\
\hline
\end{tabular}

Table 2.2: Validation requirements and activities for modeling of steady-state, normal BWR operations

\begin{tabular}{|c|c|c|c|}
\hline Phenomena & Model & $\begin{array}{r}\text { Validation } \\
\text { Tests }\end{array}$ & $\begin{array}{r}\text { Verification } \\
\text { Tests }\end{array}$ \\
\hline $\begin{array}{r}\text { Nucleate-boiling heat } \\
\text { transfer }\end{array}$ & Thom & 4 & - \\
\hline $\begin{array}{r}\text { Vapor generation (near-wall } \\
\text { condensation) }\end{array}$ & Thom with Hancox-Nicoll & - & - \\
\hline Void content & Integral effect & 7 & - \\
\hline Void drift & Lahey-Moody & 8.1 & - \\
\hline $\begin{array}{r}\text { Subcooled-boiling heat } \\
\text { transfer }\end{array}$ & \multicolumn{3}{|c|}{ (see PWR steady state) } \\
\hline Single-phase convection & \multicolumn{3}{|c|}{ (see PWR steady state) } \\
\hline $\begin{array}{r}\text { Single-phase grid spacer } \\
\text { pressure loss }\end{array}$ & \multicolumn{3}{|c|}{ (see PWR steady state) } \\
\hline $\begin{array}{r}\text { Two-phase grid spacer } \\
\text { pressure loss }\end{array}$ & Form loss & 5.3 .1 & - \\
\hline Single-phase wall shear & \multicolumn{3}{|c|}{ (see PWR steady state) } \\
\hline Two-phase wall shear & Multiplier by Wallis & 5.3 .1 & - \\
\hline $\begin{array}{r}\text { Grid heat transfer } \\
\text { enhancement }\end{array}$ & \\
\hline $\begin{array}{r}\text { Single-phase turbulent } \\
\text { mixing }\end{array}$ & \multicolumn{3}{|c|}{ (see PWR steady state) } \\
\hline Two-phase turbulent mixing & Mixing-length theory & 8.1 & - \\
\hline Pressure-directed cross flow & \multicolumn{3}{|c|}{$\begin{array}{l}0.1 \\
\text { (see PWR steady state) }\end{array}$} \\
\hline
\end{tabular}


Table 2.3: Validation requirements and activities for modeling of CIPS

\begin{tabular}{|c|c|c|c|}
\hline Phenomena & Model & $\begin{array}{r}\text { Validation } \\
\text { Tests }\end{array}$ & $\begin{array}{r}\text { Verification } \\
\text { Tests }\end{array}$ \\
\hline Grid TKE enhancement & - & - & 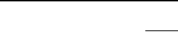 \\
\hline $\begin{array}{r}\text { Axial and azimuthal rod } \\
\text { heat transfer }\end{array}$ & Conduction equation & - & - \\
\hline $\begin{array}{r}\text { Subcooled-boiling heat } \\
\text { transfer }\end{array}$ & \multicolumn{3}{|c|}{ (see PWR steady state) } \\
\hline Single-phase wall shear & \multicolumn{3}{|c|}{ (see PWR steady state) } \\
\hline Single-phase convection & \multicolumn{3}{|c|}{ (see PWR steady state) } \\
\hline $\begin{array}{r}\text { Grid heat transfer } \\
\text { enhancement }\end{array}$ & \multicolumn{3}{|c|}{ (see PWR steady state) } \\
\hline $\begin{array}{r}\text { Single-phase turbulent } \\
\text { mixing }\end{array}$ & \multicolumn{3}{|c|}{ (see PWR steady state) } \\
\hline
\end{tabular}

Table 2.4: Validation requirements and activities for modeling of DNBR margin

\begin{tabular}{|c|c|c|c|}
\hline Phenomena & Model & $\begin{array}{r}\text { Validation } \\
\text { Tests }\end{array}$ & $\begin{array}{r}\text { Verification } \\
\text { Tests }\end{array}$ \\
\hline \multirow{4}{*}{$\mathrm{CHF}$} & $\mathrm{W}-3$ & 9.1 & 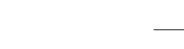 \\
\hline & Biasi & - & - \\
\hline & Bowring & - & 9.2 .1 \\
\hline & Groenveld & - & 9.2 .2 \\
\hline Natural circulation & Integral effect & 11 & - \\
\hline Single-phase convection & \multicolumn{3}{|c|}{ (see PWR steady state) } \\
\hline $\begin{array}{r}\text { Subcooled-boiling heat } \\
\text { transfer }\end{array}$ & \multicolumn{3}{|c|}{ (see PWR steady state) } \\
\hline $\begin{array}{r}\text { Nucleate-boiling heat } \\
\text { transfer }\end{array}$ & \multicolumn{3}{|c|}{ (see BWR steady state) } \\
\hline $\begin{array}{r}\text { Vapor generation (near-wall } \\
\text { condensation) }\end{array}$ & \multicolumn{3}{|c|}{ (see BWR steady state) } \\
\hline Void drift & \multicolumn{3}{|c|}{ (see BWR steady state) } \\
\hline Pressure-directed cross flow & \multicolumn{3}{|c|}{ (see PWR steady state) } \\
\hline
\end{tabular}


Table 2.5: Validation requirements and activities for modeling of RIA

\begin{tabular}{|c|c|c|c|}
\hline Phenomena & Model & $\begin{array}{r}\text { Validation } \\
\text { Tests }\end{array}$ & $\begin{array}{r}\text { Verification } \\
\text { Tests }\end{array}$ \\
\hline $\mathrm{CHF}$ & \multicolumn{3}{|c|}{ (see DNBR margin analysis) } \\
\hline Transition boiling & Bjornard and Griffith & - & - \\
\hline Radiative heat transfer & Sun, Gonzalez, and Tien & - & - \\
\hline Single-phase convection & \multicolumn{3}{|c|}{ (see PWR steady state) } \\
\hline $\begin{array}{r}\text { Subcooled-boiling heat } \\
\text { transfer }\end{array}$ & \multicolumn{3}{|c|}{ (see PWR steady state) } \\
\hline $\begin{array}{r}\text { Nucleate-boiling heat } \\
\text { transfer }\end{array}$ & \multicolumn{3}{|c|}{ (see BWR steady state) } \\
\hline Fast transient & Integral effect & 9.1 .2 & - \\
\hline
\end{tabular}




\section{CHAPTER 3}

\section{TEST DESCRIPTIONS}

This chapter presents an overview of all experiments that were used for CTF validation work.

\subsection{PSBT}

The PWR Sub-channel and Bundle Tests (PSBT) experiments include both single-channel and rod-bundle geometries as well as steady state and transient operating conditions. The PSBT Specifications[3] organizes the experiments into two phases with different subcategories.

- Phase I: Void Distribution Benchmark

- Exercise 1: Steady-state single subchannel benchmark

- Exercise 2: Steady-state bundle benchmark

- Exercise 3: Transient bundle benchmark

- Exercise 4: Pressure drop benchmark

- Phase II: DNB Benchmark

- Exercise 1: Steady-state fluid temperature benchmark

- Exercise 2: Steady-state DNB benchmark

- Exercise 3: Transient DNB benchmark

Tests from Phase I, Exercises 2 were utilized for these validation cases. Specifically, Test Series 5, 6, and 7 were used for CTF validation activities in this document. All of these test cases were rod-bundle geometry, with slightly different configurations. These configurations are detailed in Figure 3.1. Main differences included the power shapes and the placement of a central 


\begin{tabular}{|c|c|c|c|}
\hline Item & \multicolumn{3}{|c|}{ Data } \\
\hline Assembly & 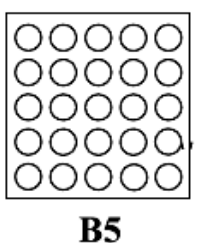 & 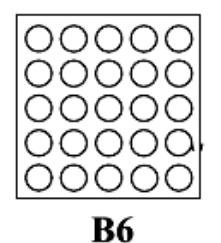 & \begin{tabular}{|c|}
00000 \\
00000 \\
00 雪00 \\
0000 \\
00000 \\
B7
\end{tabular} \\
\hline Rods array & $5 \times 5$ & $5 \times 5$ & $5 \times 5$ \\
\hline Number of heated rods & 25 & 25 & 24 \\
\hline Number of thimble rods & 0 & 0 & 1 \\
\hline Heated rod outer diameter $(\mathrm{mm})$ & 9.50 & 9.50 & 9.50 \\
\hline Thimble rod outer diameter $(\mathrm{mm})$ & - & - & 12.24 \\
\hline Heated rods pitch $(\mathrm{mm})$ & 12.60 & 12.60 & 12.60 \\
\hline Axial heated length (mm) & 3658 & 3658 & 3658 \\
\hline Flow channel inner width $(\mathrm{mm})$ & 64.9 & 64.9 & 64.9 \\
\hline Radial power shape & A & A & B \\
\hline Axial power shape & Uniform & Cosine & Cosine \\
\hline Number of MV spacers & 7 & 7 & 7 \\
\hline Number of NMV spacers & 2 & 2 & 2 \\
\hline Number of simple spacers & 8 & 8 & 8 \\
\hline MV spacer location $(\mathrm{mm})$ & \multicolumn{3}{|c|}{$471,925,1378,1832,2285,2739,3247$} \\
\hline NMV spacer location (mm) & \multicolumn{3}{|c|}{$2.5,3755$} \\
\hline Simple spacer location $(\mathrm{mm})$ & \multicolumn{3}{|c|}{$237,698,1151,1605,2059,2512,2993,3501$} \\
\hline
\end{tabular}

Figure 3.1: Summary of PSBT rod-bundle configurations for CTF validation activities 
Table 3.1: PSBT radial peaking factors: Layout A

\begin{tabular}{|l|l|l|l|l|}
\hline 0.9403 & 0.9403 & 0.9403 & 0.9403 & 0.9403 \\
\hline 0.9403 & 1.1062 & 1.1062 & 1.1062 & 0.9403 \\
\hline 0.9403 & 1.1062 & 1.1062 & 1.1062 & 0.9403 \\
\hline 0.9403 & 1.1062 & 1.1062 & 1.1062 & 0.9403 \\
\hline 0.9403 & 0.9403 & 0.9403 & 0.9403 & 0.9403 \\
\hline
\end{tabular}

unheated guide tube in Assembly B7. Test Series 5 used the B5 configuration, Test Series 6 used the B6 configuration, and Test Series 7 used the B7 configuration.

The two radial power profile shapes given in Figure 3.1 are shown in Tables 3.1 and 3.2. The specification[3] provides an axial power profile table to be used to represent the cosine power shape, which is given in Table 3.3.

There are three types of spacer grids contained within the PSBT B5 bundle. There are 7 Mixing Vane (MV) spacers, 2 Non-Mixing Vane (NMV) spacers, and 8 simple spacers. Spacer grid locations are shown in Figure 3.1. The grids were modeled using the loss-coefficients provided in the PSBT specification[3]; the simple spacers had a loss coefficient of 0.4 , the NMVs had a loss coefficient of 0.7 , and the MVs had a loss coefficient of 1.0.

Between the three test series selected for validation studies, over 200 experiments were run as part of the experiments. For this validation work, only the cases with open, publicly available data were used, which results in 35 tests being used. The test names are summarized in Table 3.4 .

Operating conditions for the three tests series are given in Tables 3.5, 3.6, and 3.7.

The average void in the four channels surrounding the central rod was the measured quantity of interest in the steady-state tests (Test Series 5, 6, and 7). These measurements were made via X-ray densitometer measurements at three different axial locations; $2.216 \mathrm{~m}, 2.269 \mathrm{~m}$, and $3.177 \mathrm{~m}$ from Beginning of Heated Length (BOHL). The estimated accuracy of the void fraction measurements was $4 \%$ void. Additional information on bundle manufacturing tolerances as well as operating condition uncertainties are given in the test specifications. 
Table 3.2: PSBT radial peaking factors: Layout B

\begin{tabular}{|l|l|l|l|l|}
\hline 0.9838 & 0.9838 & 0.9838 & 0.9838 & 0.9838 \\
\hline 0.9838 & 1.1574 & 1.1574 & 1.1574 & 0.9838 \\
\hline 0.9838 & 1.1574 & 0.0000 & 1.1574 & 0.9838 \\
\hline 0.9838 & 1.1574 & 1.1574 & 1.1574 & 0.9838 \\
\hline 0.9838 & 0.9838 & 0.9838 & 0.9838 & 0.9838 \\
\hline
\end{tabular}

Table 3.3: PSBT Cosine Axial Power Profile

\begin{tabular}{rrr}
\hline & node & ratio \\
\hline (Bottom) & 1 & 0.42 \\
2 & 0.47 \\
3 & 0.56 \\
4 & 0.67 \\
5 & 0.80 \\
6 & 0.94 \\
7 & 1.08 \\
8 & 1.22 \\
9 & 1.34 \\
& 10 & 1.44 \\
11 & 1.51 \\
12 & 1.55 \\
13 & 1.55 \\
14 & 1.51 \\
15 & 1.44 \\
16 & 1.34 \\
& 17 & 1.22 \\
18 & 1.08 \\
& 19 & 0.94 \\
20 & 0.80 \\
21 & 0.67 \\
22 & 0.56 \\
23 & 0.47 \\
& 24 & 0.42 \\
\hline Top) & &
\end{tabular}


Table 3.4: PSBT tests modeled for the CTF validation study

\begin{tabular}{ccc}
\hline Series 5 & Series 6 & Series 7 \\
\hline 51221 & 61121 & 71121 \\
51222 & 61122 & 71122 \\
52111 & 61451 & 71341 \\
52112 & 61452 & 71342 \\
52332 & 62441 & 72221 \\
52442 & 62442 & 73121 \\
53441 & 63452 & 73451 \\
53442 & 64561 & 73452 \\
54562 & 64562 & 74561 \\
56321 & 66561 & 74562 \\
56322 & 66562 & 76321 \\
56552 & & 76322 \\
\hline
\end{tabular}

Table 3.5: PSBT Test Series 5 Boundary Conditions

\begin{tabular}{rrrrr}
\hline $\begin{array}{r}\text { Run } \\
-\end{array}$ & $\begin{array}{r}\text { Pressure } \\
\text { MPa }\end{array}$ & $\begin{array}{r}\text { Mass Flux } /\left(\mathrm{m}^{2}-\mathrm{hr}\right) \times 10^{-6} \\
\mathrm{~kW}\end{array}$ & $\begin{array}{r}\text { Power } \\
\text { Inlet Temperature } \\
{ }^{\circ} \mathrm{C}\end{array}$ \\
\hline 51221 & 16.50 & 11.00 & 3000 & 292.3 \\
51222 & 16.50 & 10.98 & 2998 & 297.3 \\
52111 & 14.53 & 15.08 & 3296 & 291.9 \\
52112 & 14.52 & 14.98 & 3294 & 296.8 \\
52332 & 14.69 & 7.94 & 2523 & 287.8 \\
52442 & 14.71 & 4.99 & 2000 & 263.0 \\
53441 & 12.28 & 5.00 & 2014 & 247.9 \\
53442 & 12.27 & 5.00 & 2013 & 257.7 \\
54562 & 9.83 & 2.02 & 1016 & 214.3 \\
56321 & 4.82 & 7.87 & 3000 & 173.5 \\
56322 & 4.81 & 7.86 & 3000 & 183.6 \\
56552 & 4.92 & 2.00 & 1028 & 159.1 \\
\hline
\end{tabular}


Table 3.6: PSBT Test Series 6 Boundary Conditions

\begin{tabular}{rrrrr}
\hline $\begin{array}{r}\text { Run } \\
-\end{array}$ & $\begin{array}{r}\text { Pressure } \\
\mathrm{MPa}\end{array}$ & $\begin{array}{r}\text { Mass Flux } \\
\mathrm{kg}\left(\mathrm{m}^{2}-\mathrm{hr}\right) \times 10^{-6}\end{array}$ & $\begin{array}{r}\text { Power } \\
\mathrm{kW}\end{array}$ & $\begin{array}{r}\text { Inlet Temperature } \\
{ }^{\circ} \mathrm{C}\end{array}$ \\
\hline \hline 61121 & 16.43 & 15.16 & 3372 & 301.5 \\
61122 & 16.43 & 15.17 & 3376 & 306.7 \\
61451 & 16.58 & 5.20 & 1914 & 267.4 \\
61452 & 16.58 & 5.20 & 1915 & 272.5 \\
62441 & 14.71 & 5.16 & 2415 & 223.5 \\
62442 & 14.71 & 5.12 & 2412 & 228.4 \\
63452 & 12.28 & 5.00 & 1920 & 262.5 \\
64561 & 9.85 & 2.05 & 973 & 192.6 \\
64562 & 9.85 & 2.05 & 972 & 213.0 \\
66561 & 4.94 & 2.02 & 979 & 144.0 \\
66562 & 4.94 & 2.00 & 979 & 158.5 \\
\hline
\end{tabular}

Table 3.7: PSBT Test Series 7 Boundary Conditions

\begin{tabular}{rrrrr}
\hline Run & $\begin{array}{r}\text { Pressure } \\
\text { MPa }\end{array}$ & $\begin{array}{r}\text { Mass Flux } /\left(\mathrm{m}^{2}-\mathrm{hr}\right) \times 10^{-6} \\
\mathrm{~kW}\end{array}$ & $\begin{array}{r}\text { Power } \\
\text { Inlet Temperature } \\
{ }^{\circ} \mathrm{C}\end{array}$ \\
\hline 71121 & 16.42 & 15.07 & 3385 & 301.8 \\
71122 & 16.42 & 15.07 & 3384 & 306.8 \\
71341 & 16.55 & 7.92 & 2391 & 289.4 \\
71342 & 16.55 & 7.94 & 2391 & 295.3 \\
72221 & 14.64 & 11.01 & 3503 & 272.1 \\
73121 & 12.13 & 15.20 & 3502 & 276.1 \\
73451 & 12.27 & 5.03 & 2023 & 242.8 \\
73452 & 12.27 & 5.03 & 2021 & 260.1 \\
74561 & 9.83 & 2.16 & 1023 & 196.8 \\
74562 & 9.84 & 2.16 & 1023 & 214.9 \\
76321 & 4.89 & 8.10 & 3541 & 153.5 \\
76322 & 4.87 & 8.06 & 3536 & 168.6 \\
\hline
\end{tabular}




\section{$3.2 \quad$ BFBT $8 \times 8$}

Facility Description The BWR Full-size Fine-mesh Bundle Tests (BFBT) are organized into phases and exercises as described in the BFBT specification [4].

- Phase I: Void Distribution Benchmark

- Exercise 1: Steady-state subchannel grade benchmark

- Exercise 2: Steady-state microscopic grade benchmark

- Exercise 3: Transient microscopic grade benchmark

- Exercise 4: Uncertainty analysis of the void distribution benchmark

- Phase II: Critical Power Benchmark

- Exercise 0: Steady-state pressure drop benchmark

- Exercise 1: Steady-state critical power benchmark

- Exercise 2: Transient benchmark

Tests from Phase II, Exercise 0 and Phase I, Exercise 1 are used for validation work. These tests cover single- and two-phase pressure drop as well as void content and distribution. For this validation study, 18 two-phase pressure drop (P6 tests) experiments and 10 single-phase pressure drop (P7 tests) experiments were modeled. Tests are summarized in Tables 3.8 and 3.9. Additionally, 16 of the void distribution tests are modeled. Table 3.10 shows their operating conditions.

The assembly type refers to the placement of heater rods, guide tubes, the water rod, and power configurations. Figures 3.2 and 3.3 show the geometry and rod configuration for Assembly Types $0-1,0-2$, and $0-3$. All of these tests have a uniform axial and radial heating. The only difference between the assembly types is that some heater rods are shut off in Assembly Types 0-2 and 0-3. Figure 3.4 shows the geometry and power configuration for Assembly Types 1, 2, and 3. Only tests of Assembly Type 1 are modeled in this study. Assembly Type 1 has a cosine power shape, which is shown in Table 3.11. All other assembly types modeled in this study have a uniform axial power shape. Assembly Types 1 and 4 have non-uniform radial power distributions. The Assembly Type 1 radial power distribution is shown in Figure 3.5, and the Assembly Type 4 radial power distribution is shown in Figure 3.6.

Finally, Figure 3.7 shows the rod configuration and power distribution for Assembly Types 4, C2A, C2B, and C3. Tests using Assembly type 4 are used in the void distribution tests. Assembly Type 4 replaces the four central rods with a large water rod. Assembly C2A is used in the pressure drop tests.

Table 3.13 contains the radial power profile for Assembly C2A while Table 3.12 contains the axial power profile for this bundle. Pressure tap locations are shown in Figure 3.8.

Void measurements were made in the facility using two approaches: 1) an x-ray densitometer was used at several axial locations, and 2) a CT scanner was used to obtain a fine-mesh void distribution measurement $50 \mathrm{~mm}$ above the end of heated length. The CT scanner measurements 
Table 3.8: BFBT Series P6 Boundary Conditions

\begin{tabular}{rrrrrr}
\hline Test & $\begin{array}{r}\text { Pressure } \\
\mathrm{MPa}\end{array}$ & $\begin{array}{r}\text { Inlet Temperature } \\
{ }^{\circ} \mathrm{C}\end{array}$ & $\begin{array}{r}\text { Inlet Subcooling } \\
\mathrm{kJ} / \mathrm{kg}\end{array}$ & $\begin{array}{r}\text { Flow Rate } \\
\text { ton/hr }\end{array}$ & $\begin{array}{r}\text { Power } \\
\mathrm{MW}\end{array}$ \\
\hline $\mathrm{P} 60001$ & 7.16 & 277.3 & 53.3 & 20.2 & 0.863 \\
$\mathrm{P} 60003$ & 7.16 & 277.8 & 50.8 & 20.1 & 1.521 \\
P60005 & 7.16 & 277.7 & 51.1 & 20.0 & 2.357 \\
P60007 & 7.17 & 277.8 & 51.1 & 55.0 & 2.375 \\
P60009 & 7.17 & 277.8 & 51.1 & 55.0 & 4.197 \\
P60013 & 7.16 & 278.4 & 47.2 & 69.9 & 3.022 \\
P60015 & 7.17 & 278.2 & 49.5 & 70.0 & 5.340 \\
P60017 & 7.16 & 277.8 & 51.0 & 45.1 & 1.919 \\
P60019 & 7.17 & 278.2 & 49.4 & 45.0 & 3.437 \\
P60022 & 8.64 & 291.3 & 50.7 & 20.2 & 0.837 \\
P60023 & 8.63 & 291.0 & 52.3 & 20.2 & 1.464 \\
P60024 & 8.63 & 290.9 & 52.9 & 20.2 & 2.252 \\
P60025 & 8.64 & 291.3 & 51.3 & 55.0 & 2.271 \\
P60026 & 8.64 & 291.0 & 53.0 & 55.1 & 3.975 \\
P60029 & 8.64 & 291.3 & 51.5 & 70.1 & 2.888 \\
P60030 & 8.64 & 291.2 & 51.4 & 70.2 & 5.076 \\
P60031 & 8.64 & 290.9 & 53.0 & 45.1 & 1.869 \\
P60032 & 8.63 & 291.2 & 51.3 & 45.2 & 3.262 \\
\hline
\end{tabular}

Table 3.9: BFBT Series P7 Boundary Conditions

\begin{tabular}{rrrrr}
\hline Test & $\begin{array}{r}\text { Pressure } \\
\mathrm{MPa}\end{array}$ & $\begin{array}{r}\text { Inlet Temperature } \\
{ }^{\circ} \mathrm{C}\end{array}$ & $\begin{array}{r}\text { Flow Rate } \\
\text { ton/hr }\end{array}$ & $\begin{array}{r}\text { Reynolds } \\
\times 10^{-4}\end{array}$ \\
\hline P70027 & 7.15 & 284.9 & 20.30 & 8.07 \\
P70028 & 7.16 & 285.1 & 24.90 & 9.91 \\
P70029 & 7.16 & 285.1 & 29.80 & 11.86 \\
P70030 & 7.16 & 285.7 & 34.70 & 13.82 \\
P70031 & 7.16 & 285.6 & 39.70 & 15.81 \\
P70032 & 7.16 & 285.3 & 44.60 & 17.75 \\
P70033 & 7.15 & 284.7 & 55.00 & 21.86 \\
P70034 & 7.15 & 284.8 & 59.70 & 23.74 \\
P70035 & 7.16 & 284.6 & 64.80 & 25.76 \\
P70036 & 7.15 & 284.8 & 69.90 & 27.79 \\
\hline
\end{tabular}


Table 3.10: BFBT void distribution test conditions

\begin{tabular}{rrrrrr}
\hline Test & $\begin{array}{r}\text { Assembly Type } \\
\end{array}$ & $\begin{array}{r}\text { Pressure } \\
{[\mathrm{MPa}]}\end{array}$ & $\begin{array}{r}\text { Inlet Subcooling } \\
{[\mathrm{kJ} / \mathrm{kg}]}\end{array}$ & $\begin{array}{r}\text { Flow Rate } \\
{[\text { ton } / \mathrm{h}]}\end{array}$ & $\begin{array}{r}\text { Power } \\
\text { MW }\end{array}$ \\
\hline $0011-55$ & $0-1$ & 7.180 & 52.60 & 54.03 & 1.90 \\
$0011-58$ & $0-1$ & 7.172 & 51.00 & 54.90 & 3.51 \\
$0011-61$ & $0-1$ & 7.210 & 50.90 & 54.79 & 6.44 \\
$0021-16$ & $0-2$ & 7.190 & 54.00 & 54.85 & 1.91 \\
$0021-18$ & $0-2$ & 7.171 & 49.80 & 54.90 & 3.51 \\
$0021-21$ & $0-2$ & 7.179 & 51.40 & 54.90 & 6.45 \\
$0031-16$ & $0-3$ & 7.180 & 52.40 & 54.96 & 1.92 \\
$0031-18$ & $0-3$ & 7.179 & 50.00 & 54.79 & 3.52 \\
$0031-21$ & $0-3$ & 7.171 & 49.40 & 54.90 & 6.45 \\
$1071-55$ & 1 & 7.191 & 52.80 & 54.61 & 1.92 \\
$1071-58$ & 1 & 7.158 & 50.30 & 55.07 & 3.52 \\
$1071-61$ & 1 & 7.200 & 51.80 & 54.65 & 6.48 \\
$4101-53$ & 4 & 7.181 & 52.80 & 54.65 & 1.24 \\
$4101-55$ & 4 & 7.195 & 52.90 & 54.59 & 1.92 \\
$4101-58$ & 4 & 7.152 & 50.60 & 54.58 & 3.52 \\
$4101-61$ & 4 & 7.180 & 52.50 & 54.65 & 6.48 \\
\hline
\end{tabular}

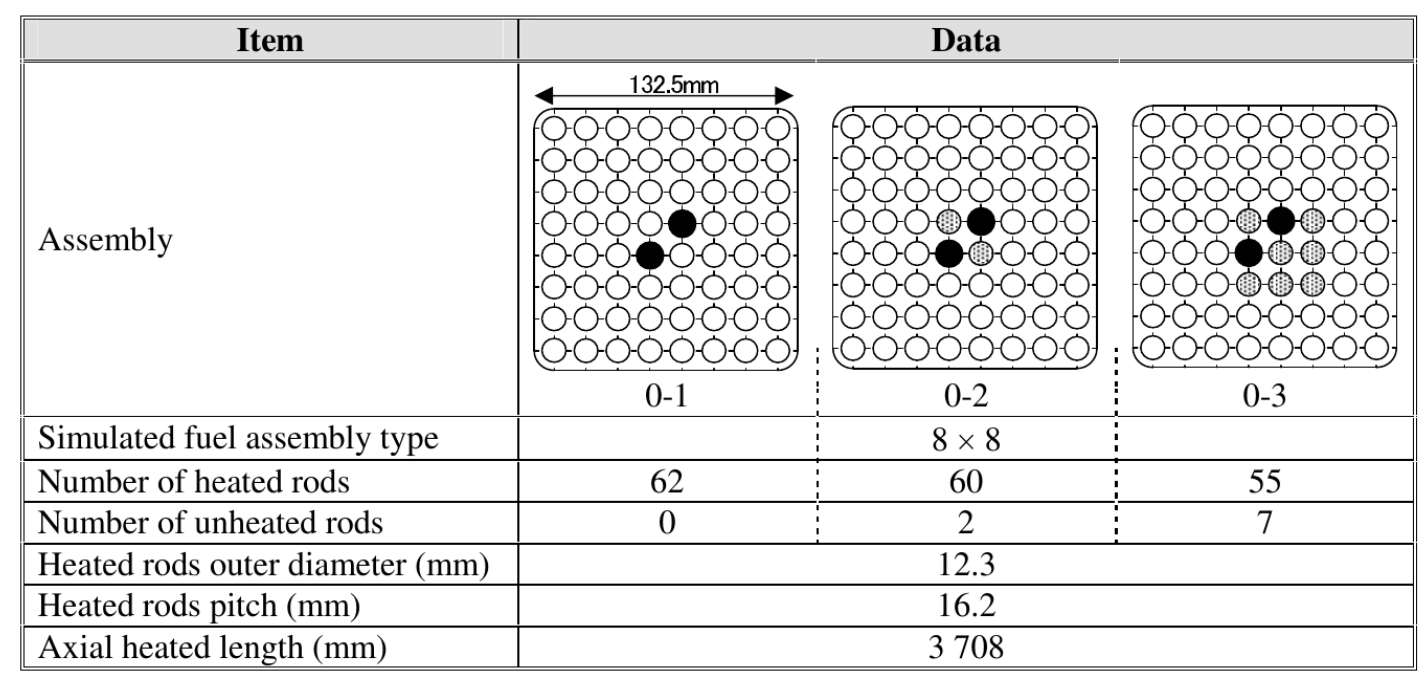

O Heated rod, $\rightarrow$ Unheated rod, $\bullet$ Water rod - no flow in water rods

Figure 3.2: Description of BFBT Assembly Types 0-1, 0-2, and 0-3 (reprinted from B. Neykov et al. NUPEC BWR Full-size Fine-mesh Bundle Test (BFBT) Benchmark. Tech. rep. NUCLEAR ENERGY AGENCY, 2006) 


\begin{tabular}{||l|c||}
\hline \multicolumn{1}{|c|}{ Item } & Data \\
\hline \hline Number of water rods & 2 \\
\hline Water rods outer diameter $(\mathrm{mm})$ & 15.0 \\
\hline Channel box inner width $(\mathrm{mm})$ & 132.5 \\
\hline Channel box corner radius $(\mathrm{mm})$ & 8.0 \\
\hline In channel flow area $\left(\mathrm{mm}^{2}\right)$ & 9781 \\
\hline Spacer type & Grid \\
\hline Number of spacers & 7 \\
\hline Spacer pressure loss coefficients & 1.2 \\
\hline Spacer location (mm) & 455, 967, 1 479, 1 991, 2 503, 3 015, 3 527 \\
& (distance from bottom of heated length to spacer bottom face) \\
\hline Radial power shape & Uniform \\
\hline Axial power shape & Uniform \\
\hline
\end{tabular}

Figure 3.3: Description of BFBT Assembly Types 0-1, 0-2, and 0-3 (continued) (reprinted from B. Neykov et al. NUPEC BWR Full-size Fine-mesh Bundle Test (BFBT) Benchmark. Tech. rep. NUCLEAR ENERGY AGENCY, 2006).

Table 3.11: BFBT Assembly Type 1 axial power distribution.

\begin{tabular}{rr}
\hline Axial level & Power factor \\
\hline 1 & 0.46 \\
2 & 0.58 \\
3 & 0.69 \\
4 & 0.79 \\
5 & 0.88 \\
6 & 0.99 \\
7 & 1.09 \\
8 & 1.22 \\
9 & 1.22 \\
10 & 1.34 \\
11 & 1.34 \\
12 & 1.40 \\
13 & 1.40 \\
14 & 1.34 \\
15 & 1.34 \\
16 & 1.22 \\
17 & 1.22 \\
18 & 1.09 \\
19 & 0.99 \\
20 & 0.88 \\
21 & 0.79 \\
22 & 0.69 \\
23 & 0.58 \\
24 & 0.46 \\
\hline
\end{tabular}




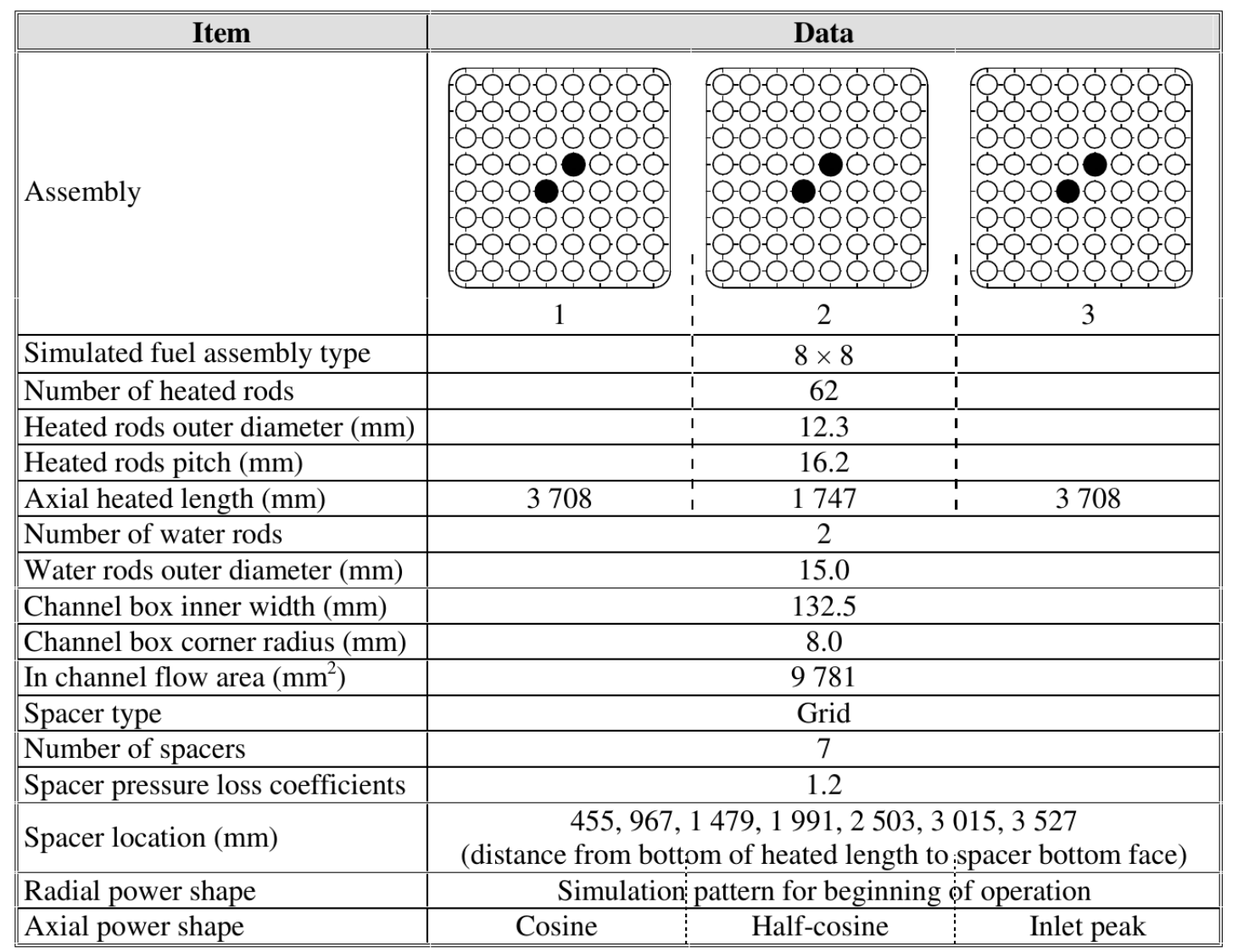

O Heated rod, $\rightarrow$ Unheated rod, $\bullet$ Water rod - no flow in water rods

Figure 3.4: Description of BFBT Assembly Types 1, 2, and 3 (reprinted from B. Neykov et al. NUPEC BWR Full-size Fine-mesh Bundle Test (BFBT) Benchmark. Tech. rep. NUCLEAR ENERGY AGENCY, 2006).

\begin{tabular}{|l:l:l:l:l:l:l:l:l|}
\hline 1.15 & 1.30 & 1.15 & 1.30 & 1.30 & 1.15 & 1.30 & 1.15 \\
\hline 1.30 & 0.45 & 0.89 & 0.89 & 0.89 & 0.45 & 1.15 & 1.30 \\
\hline 1.15 & 0.89 & 0.89 & 0.89 & 0.89 & 0.89 & 0.45 & 1.15 \\
\hline 1.30 & 0.89 & 0.89 & 0.89 & & 0.89 & 0.89 & 1.15 \\
\hline 1.30 & 0.89 & 0.89 & & 0.89 & 0.89 & 0.89 & 1.15 \\
\hline 1.15 & 0.45 & 0.89 & 0.89 & 0.89 & 0.89 & 0.45 & 1.15 \\
\hline 1.30 & 1.15 & 0.45 & 0.89 & 0.89 & 0.45 & 1.15 & 1.30 \\
\hline 1.15 & 1.30 & 1.15 & 1.15 & 1.15 & 1.15 & 1.30 & 1.15 \\
\hline
\end{tabular}

Figure 3.5: Radial power distribution applied to Assembly Type 1 (reprinted from B. Neykov et al. NUPEC BWR Full-size Fine-mesh Bundle Test (BFBT) Benchmark. Tech. rep. NUCLEAR ENERGY AGENCY, 2006). 


\begin{tabular}{|l|l|l|l|l|l|l|l|}
\hline 1.15 & 1.30 & 1.15 & 1.30 & 1.30 & 1.15 & 1.30 & 1.15 \\
\hline 1.30 & 0.45 & 0.89 & 0.89 & 0.89 & 0.45 & 1.15 & 1.30 \\
\hline 1.15 & 0.89 & 0.89 & 0.89 & 0.89 & 0.89 & 0.45 & 1.15 \\
\hline 1.30 & 0.89 & 0.89 & & & 0.89 & 0.89 & 1.15 \\
\hline 1.30 & 0.89 & 0.89 & & & 0.89 & 0.89 & 1.15 \\
\hline 1.15 & 0.45 & 0.89 & 0.89 & 0.89 & 0.89 & 0.45 & 1.15 \\
\hline 1.30 & 1.15 & 0.45 & 0.89 & 0.89 & 0.45 & 1.15 & 1.30 \\
\hline 1.15 & 1.30 & 1.15 & 1.15 & 1.15 & 1.15 & 1.30 & 1.15 \\
\hline
\end{tabular}

Figure 3.6: Radial power distribution applied to Assembly Type 4 (reprinted from B. Neykov et al. NUPEC BWR Full-size Fine-mesh Bundle Test (BFBT) Benchmark. Tech. rep. NUCLEAR ENERGY AGENCY, 2006).

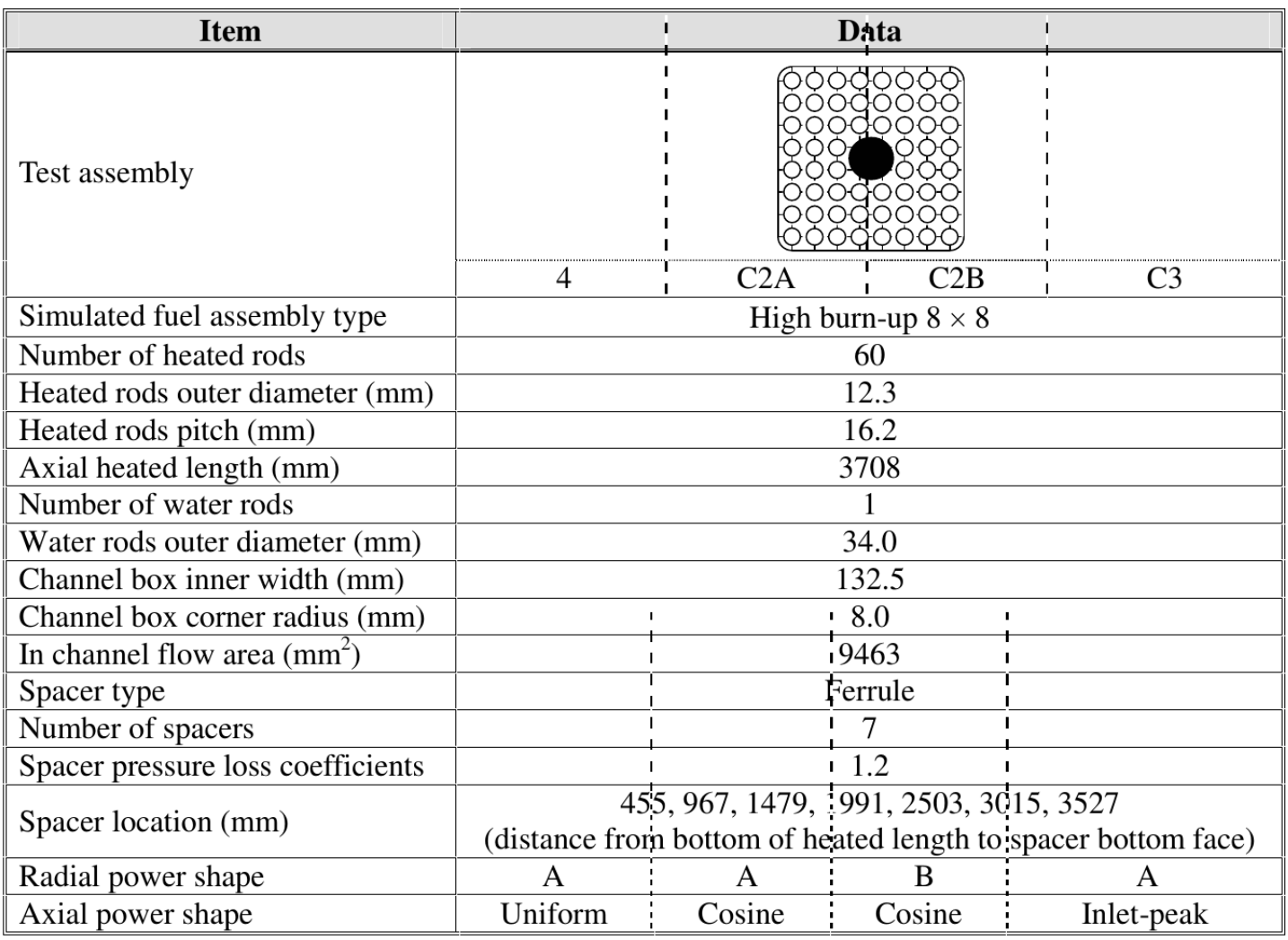

O Heated rod, $\bullet$ Water rod - no flow in water rods

A: Simulation pattern for beginning of operation.

B: Simulation pattern for middle of operation.

Figure 3.7: Description of BFBT Assembly Type 4 (reprinted from B. Neykov et al. NUPEC BWR Full-size Fine-mesh Bundle Test (BFBT) Benchmark. Tech. rep. NUCLEAR ENERGY AGENCY, 2006). 


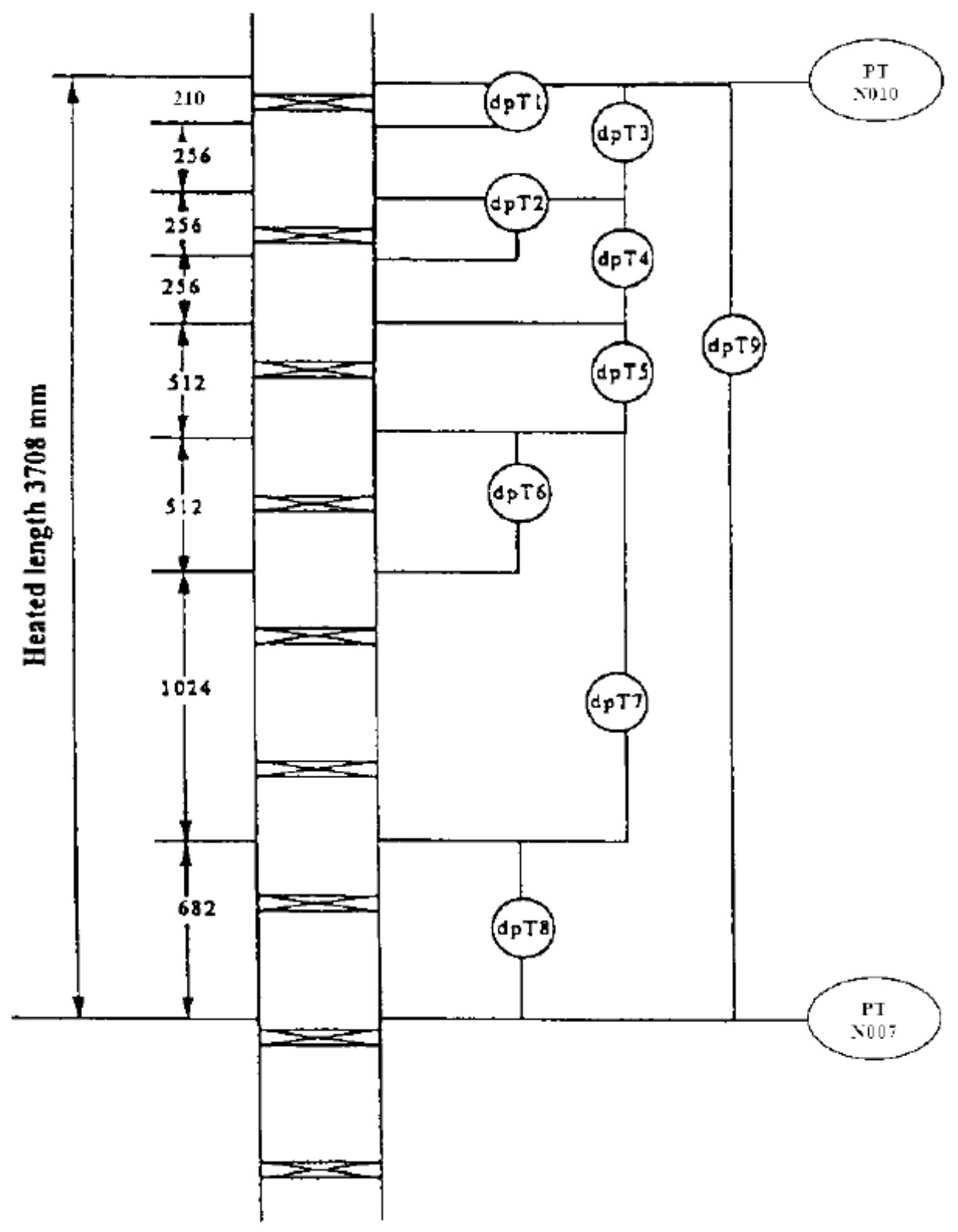

Figure 3.8: BFBT bundle pressure tap locations 
Table 3.12: BFBT C2A Bundle Axial Power Distribution

\begin{tabular}{ccc}
\hline & node & ratio \\
\hline (bottom) & 1 & 0.46 \\
& 2 & 0.58 \\
& 3 & 0.69 \\
& 4 & 0.79 \\
& 5 & 0.88 \\
& 6 & 0.99 \\
& 7 & 1.09 \\
& 8 & 1.22 \\
& 9 & 1.22 \\
& 10 & 1.34 \\
& 11 & 1.34 \\
& 12 & 1.40 \\
& 13 & 1.40 \\
& 14 & 1.34 \\
& 15 & 1.34 \\
& 16 & 1.22 \\
& 17 & 1.22 \\
& 18 & 1.09 \\
& 19 & 0.99 \\
& 20 & 0.88 \\
& 21 & 0.79 \\
& 22 & 0.69 \\
& 23 & 0.58 \\
(top) & 24 & 0.46 \\
\hline & &
\end{tabular}


Table 3.13: BFBT C2A Bundle Radial Power Distribution

\begin{tabular}{|l|l|l|l|l|l|l|l|}
\hline 1.15 & 1.30 & 1.15 & 1.30 & 1.30 & 1.15 & 1.30 & 1.15 \\
\hline 1.30 & 0.45 & 0.89 & 0.89 & 0.89 & 0.45 & 1.15 & 1.30 \\
\hline 1.15 & 0.89 & 0.89 & 0.89 & 0.89 & 0.89 & 0.45 & 1.15 \\
\hline 1.30 & 0.89 & 0.89 & - & - & 0.89 & 0.89 & 1.15 \\
\hline 1.30 & 0.89 & 0.89 & - & - & 0.89 & 0.89 & 1.15 \\
\hline 1.15 & 0.45 & 0.89 & 0.89 & 0.89 & 0.89 & 0.45 & 1.15 \\
\hline 1.30 & 1.15 & 0.45 & 0.89 & 0.89 & 0.45 & 1.15 & 1.30 \\
\hline 1.15 & 1.30 & 1.15 & 1.15 & 1.15 & 1.15 & 1.30 & 1.15 \\
\hline
\end{tabular}

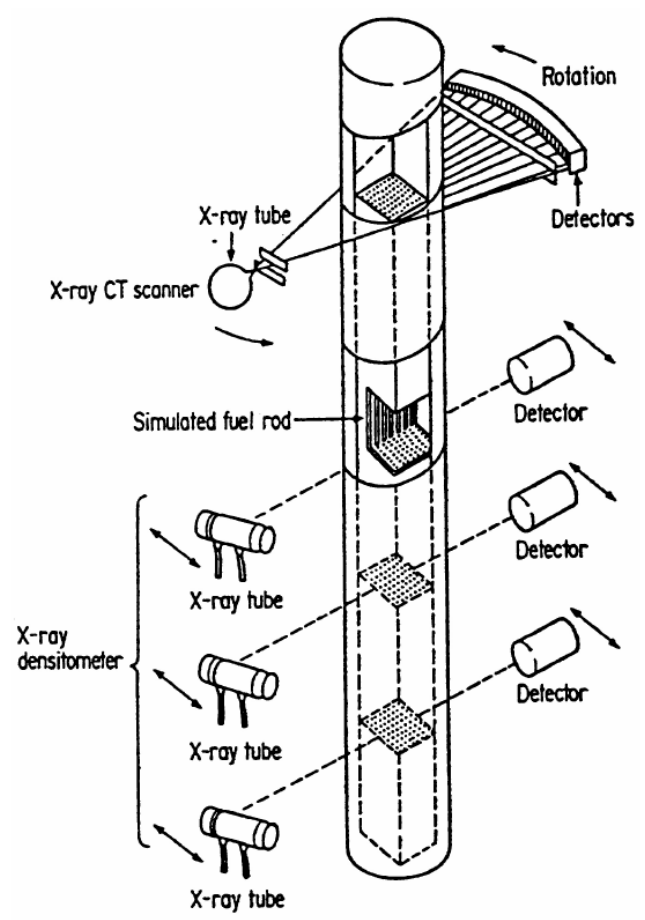

Figure 3.9: Depiction of void measurement locations and techniques in BFBT facility (reprinted from B. Neykov et al. NUPEC BWR Full-size Fine-mesh Bundle Test (BFBT) Benchmark. Tech. rep. NUCLEAR ENERGY AGENCY, 2006. 
are used for comparison with CTF in this study. Figure 3.9 shows a schematic of where different void measurements were made in the facility.

The CT scanner worked by sweeping over the bundle at a fixed axial plane to obtain void measurements in small "pixels" as small as $0.3 \times 0.3 \mathrm{~mm}$. The refined measurements have been used for CFD validation work, but the measurements were also post-processed to create a set of subchannel-averaged void measurements for comparison with subchannel codes. The measurements were made several times for the same operating conditions and were time-averaged to avoid the effects of two-phase flow oscillations.

The BFBT specification [4] states that the subchannel accuracy is estimated to be $3 \%$ and the bundle average void is estimated to be $2 \%$ (as will be shown when analyzing symmetry of the results, the measurement error is likely much higher than this).

CTF Model Figure 3.10 shows the flow area and wetted perimeter for the channels in the CTF model for Assembly Types 0-1, 0-2, 0-3, and 1. It also shows the channel indexing scheme employed in the model. Note that the center channel is indexed as Channel 81. For the Assembly Type 4, Channel 81 is simply removed from the model along with the four surrounding rods. A large water rod is positioned in their place and the flow areas are modified as shown in Figure 3.11 .

Spacer grid locations are provided in Figures 3.2, 3.3, 3.4, and 3.7. These are ferrule-type spacers with circular tubes (ferrules) to guide each heated rod as well as the central water rod. An example of this type of grid is shown in Figure 3.12.

The loss coefficients for this type of spacer grid are calculated using the approach of Shiralkar and Radcliffe [5]. Details of the calculation are shown in [6]. Figure 3.13 shows the radial loss coefficient distribution in the bundle.

The CTF friction correlation is used [2] along with a single-phase mixing coefficient of 0.007 and a Beus two-phase multiplier, $\Theta_{M}$, of 5.0. The cases are run with and without the droplet field enabled to test the effect on void content and distribution. Results of this study are shown in Section 8.1.2. When the droplet field is disabled, it means that there is no entrainment or deentrainment and the interfacial drag and heat transfer between droplets and vapor is eliminated in the solution. The sensitivity study shows that results are improved when the droplet field is disabled, and so the remainder of the void-distribution validation work is performed with the droplet field disabled.

The axial mesh is set up so that the height of a spacer grid occupies one full level. The average axial mesh cell height is $3.635 \mathrm{~cm}$. Only heater rods are explicitly modeled; unheated objects, such as guide tubes and the fuel canister, are not modeled, as they have no impact on the solution in a steady-state solve. Furthermore, the power is set as a heat flux boundary condition, which disables the internal rod temperature solution. 


\begin{tabular}{|c|c|c|c|c|c|c|c|c|}
\hline 1 & 2 & 3 & 4 & 5 & 6 & 7 & 8 & 9 \\
\hline 10 & 11 & 12 & 13 & 14 & 15 & 16 & 17 & 18 \\
\hline 19 & 20 & 21 & 22 & 23 & 24 & 25 & 26 & 27 \\
\hline 28 & 29 & 30 & 31 & 32 & 33 & 34 & 35 & 36 \\
\hline 37 & 38 & 39 & 40 & 81 & 41 & 42 & 43 & 44 \\
\hline 45 & 46 & 47 & 48 & 49 & 50 & 51 & 52 & 53 \\
\hline 54 & 55 & 56 & 57 & 58 & 59 & 60 & 61 & 62 \\
\hline 63 & 64 & 65 & 66 & 67 & 68 & 69 & 70 & 71 \\
\hline 72 & 73 & 74 & 75 & 76 & 77 & 78 & 79 & 80 \\
\hline
\end{tabular}

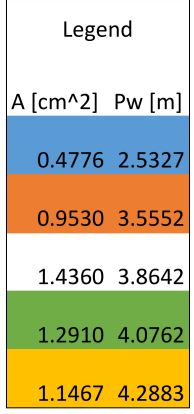

Figure 3.10: Map of the channel flow areas and wetted perimeters for Assembly Types 0-1, 0-2, $0-3$, and 1 .

\begin{tabular}{|c|c|c|c|c|c|c|c|c|}
\hline 1 & 2 & 3 & 4 & 5 & 6 & 7 & 8 & 9 \\
\hline 10 & 11 & 12 & 13 & 14 & 15 & 16 & 17 & 18 \\
\hline 19 & 20 & 21 & 22 & 23 & 24 & 25 & 26 & 27 \\
\hline 28 & 29 & 30 & 31 & 32 & 33 & 34 & 35 & 36 \\
\hline 37 & 38 & 39 & 40 & & 41 & 42 & 43 & 44 \\
\hline 45 & 46 & 47 & 48 & 49 & 50 & 51 & 52 & 53 \\
\hline 54 & 55 & 56 & 57 & 58 & 59 & 60 & 61 & 62 \\
\hline 63 & 64 & 65 & 66 & 67 & 68 & 69 & 70 & 71 \\
\hline 72 & 73 & 74 & 75 & 76 & 77 & 78 & 79 & 80 \\
\hline
\end{tabular}

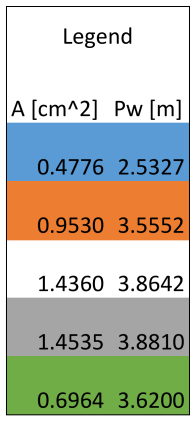

Figure 3.11: Map of the channel flow areas and wetted perimeters for Assembly Type 4. 

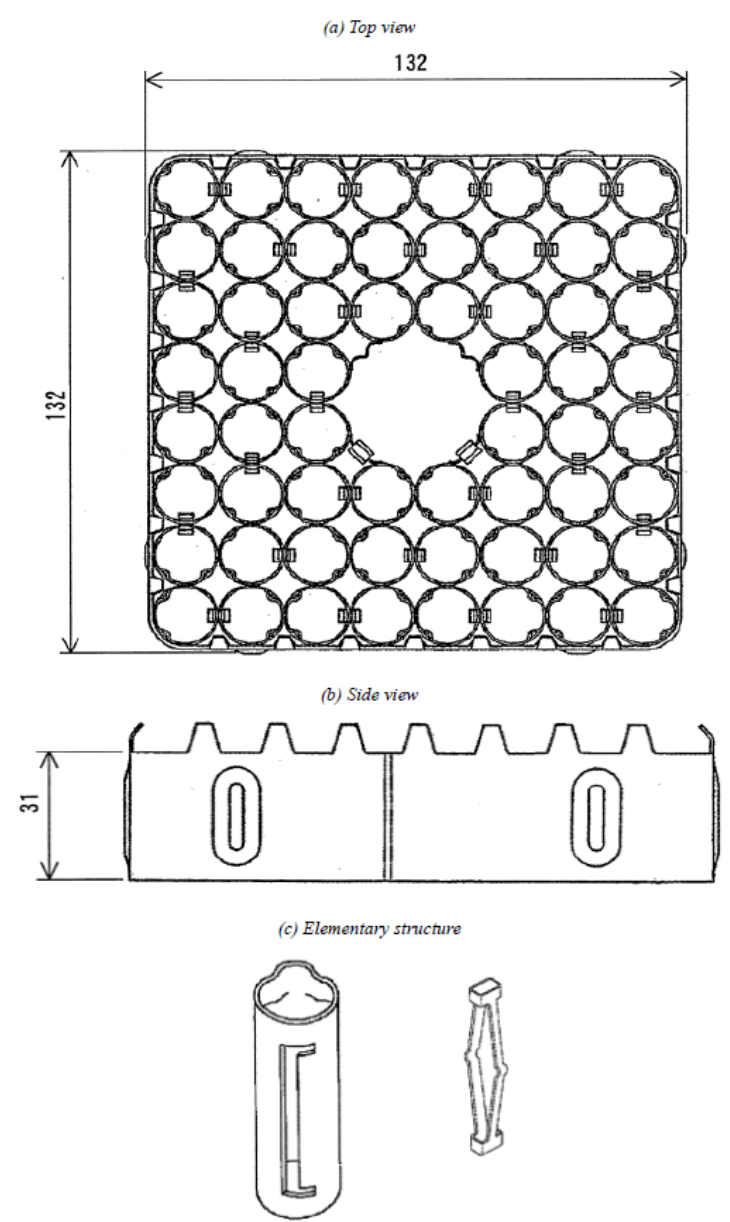

Figure 3.12: $\quad$ BFBT $\mathrm{C} 2 \mathrm{~A}$ ferrule grid geometry (reprinted from B. Neykov et al. NUPEC BWR Full-size Fine-mesh Bundle Test (BFBT) Benchmark. Tech. rep. NUCLEAR ENERGY AGENCY, 2006). 


\begin{tabular}{|c|c|c|c|c|c|c|c|c|}
\hline 72 & 73 & 74 & 75 & 76 & 77 & 78 & 79 & 80 \\
\hline 63 & 64 & 65 & 66 & 67 & 68 & 69 & 70 & 71 \\
\hline 54 & 55 & 56 & 57 & 58 & 59 & 60 & 61 & 62 \\
\hline 45 & 46 & 47 & 48 & 49 & 50 & 51 & 52 & 53 \\
\hline 37 & 38 & 39 & 40 & & 41 & 42 & 43 & 44 \\
\hline 28 & 29 & 30 & 31 & 32 & 33 & 34 & 35 & 36 \\
\hline 19 & 20 & 21 & 22 & 23 & 24 & 25 & 26 & 27 \\
\hline 10 & 11 & 12 & 13 & 14 & 15 & 16 & 17 & 18 \\
\hline 1 & 2 & 3 & 4 & 5 & 6 & 7 & 8 & 9 \\
\hline \multicolumn{9}{|c|}{ Legend (loss coefficients of color): } \\
\hline \multicolumn{2}{|c|}{$\mathrm{K}=1.348$} & \multicolumn{2}{|c|}{$K=1.222$} & $K=1.304$ & & 0.778 & \multicolumn{2}{|c|}{$K=0.856$} \\
\hline \multicolumn{2}{|c|}{$K=1.278$} & \multicolumn{2}{|c|}{$\mathrm{K}=1.606$} & $K=0.748$ & \multicolumn{2}{|c|}{$\mathrm{K}=0.926$} & \multicolumn{2}{|c|}{$K=1.475$} \\
\hline
\end{tabular}

Figure 3.13: Map of channels in BFBT cases with loss coefficient for each channel as calculated by Shiralkar and Radcliffe (B.S. Shiralkar and D.W. Radcliffe. An experimental and analytical study of the synthesis of grid spacer loss coefficients. Tech. rep. NEDE-13181. General Electric, 1971). 


\section{$3.3 \quad$ PNNL $2 \times 6$}

These two experiments, both made in a 2-by-6 apparatus with the same geometry, were meant to provide benchmark data to evaluate the ability of codes to account for the effects of buoyancy on flow patterns. They contained fixed inlet flow, which was then re-distributed across the bundle by temperature gradients due to differing powers on the two sides. Specifically, for both problems, one half of the assembly (6 rods) was given power while the other half remained unheated. The apparatus contained 9 windows at 6 -inch intervals along its 4 -ft heated length. At these elevations, a Laser Doppler Anemometer (LDA) was used to measure velocities along lines at 3 locations ( $\mathrm{Y}=-0.581,0.0,0.581)$, which correspond to the three subchannel rows along the 2-rod dimension. Additionally, thermocouple measurements were made in several subchannel centers at these same elevations.

The CTF model contained 12 rods and 21 subchannels arranged as shown in Figure 3.14. The numbering from the steady-state test reference[7] was used, in contrast to that in the transient test reference[8], where the subchannel numbering began in the middle row. The axial meshing of the test section is shown in Figure 3.15. Pertinent geometric information can be found in Table 3.14.

Along with the $4 \mathrm{ft}$ heated length of the rods, 6 inches of unheated length above and below were included. There were a total of 30 axial nodes, each 2 inches long. The second friction option was used (irfc=2), as well as the Rogers and Roseheart single-phase mixing coefficient (imix =2). As these are both single phase cases, options for liquid entrainment, two-phase mixing, and void drift were turned off or set as the defaults.

The steady-state problem was modeled using the boundary conditions found in Table 3.15. The transient problem had the boundary conditions shown in Table 3.16. However, note that convergence problems were experienced for the low pressure case, so the pressure was increased to 60 psi for the transient case. Since the test contained single-phase liquid and low temperature, it is anticipated this change to pressure will have an insignificant impact on results. Additionally, to ensure the case started at steady state, the transient flow ramp was not started until 300 seconds into the CTF simulation.

Table 3.14: Subchannel geometry data for the PNNL 2x6 facility

\begin{tabular}{rr}
\hline Parameter & Value \\
\hline Rod diameter & 0.475 in \\
Rod pitch & 0.575 in \\
Rod-wall spacing & 0.350 in \\
\hline
\end{tabular}




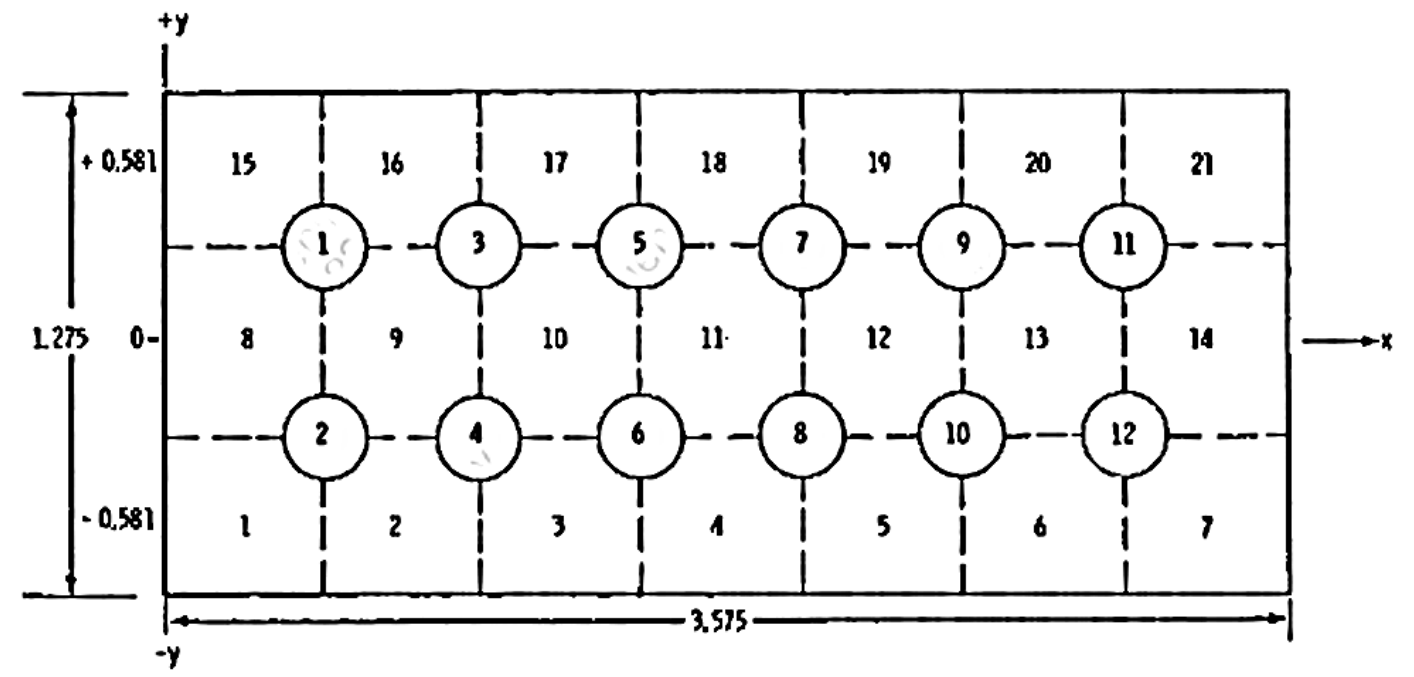

Figure 3.14: Cross-sectional geometry of the PNNL 2x6 test section

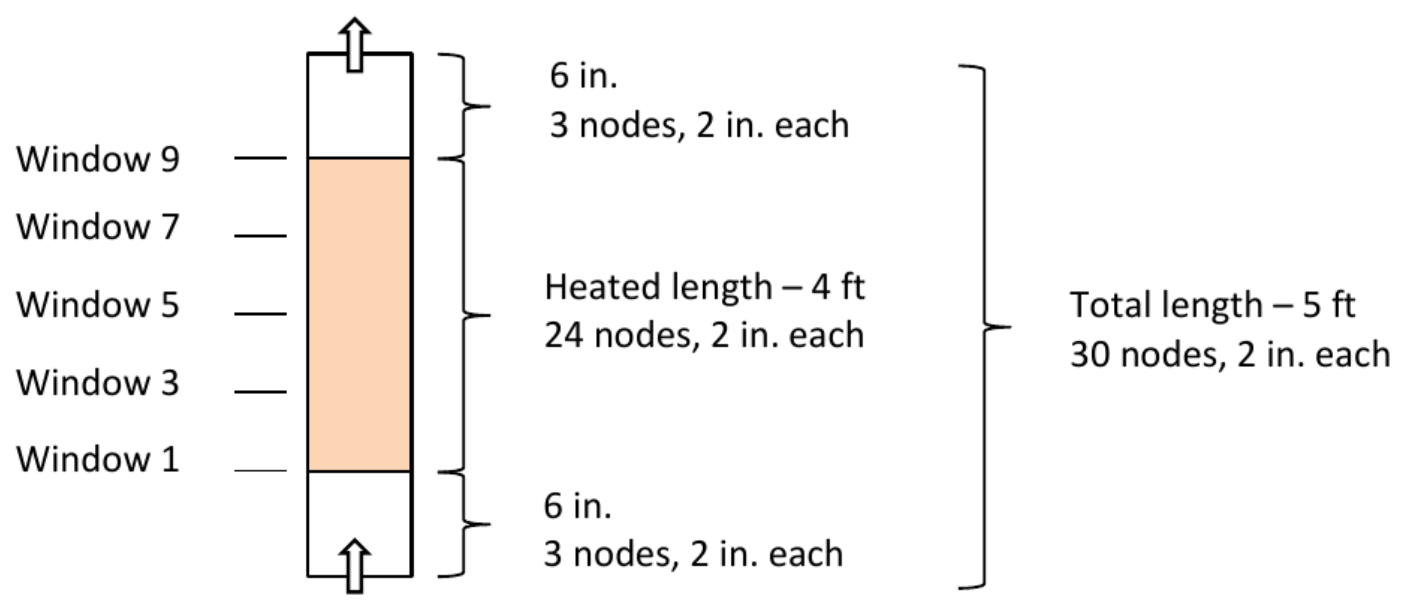

Figure 3.15: Axial schematic of the PNNL 2x6 facility and CTF meshing (units shown in inches)

Table 3.15: Boundary conditions for PNNL 2x6 steady-state test

\begin{tabular}{rr}
\hline Boundary Condition & Value \\
\hline Pressure $(\mathrm{psi})$ & 60 \\
Inlet temperature (F) & 60.6 \\
Outlet temperature (F) & 93.1 \\
Flow rate (gpm) & 1.25 \\
Power per rod [kW] & 0.91 \\
\hline
\end{tabular}


Table 3.16: Boundary conditions for PNNL 2x6 transient test (Case 22)

\begin{tabular}{rr}
\hline Boundary Condition & Value \\
\hline Pressure $(\mathrm{psi})$ & 20 \\
Inlet temperature (C) & 22.9 \\
Outlet temperature (C) & 67.8 \\
Initial flow rate (gpm) & 1.63 \\
Final flow rate (gpm) & 0.55 \\
Flow ramp start (s) & 0.0 \\
Flow ramp end (s) & 45.0 \\
Power per rod (kW) & 0.91 \\
\hline
\end{tabular}




\section{$3.4 \quad$ CE $5 \times 5$}

In the early 1980s, Combustion Engineering (CE) teamed up with Columbia University to perform rod-bundle experiments to provide insight on the efficacy of single- and two-phase heat transfer models that are typically used for predicting steaming rate and crud buildup[9]. Two $5 \times 5$ rod-bundle tests (named Test 74 and Test 75) were performed at the Columbia University Heat Transfer Research Facility, which were designed to simulate the peripheral region of adjacent CE 14x14 fuel bundles. These tests were prompted after heavy crud deposits and fuel failures were observed on peripheral rods for bundles in Maine Yankee cycle 4.

The experiments were run at prototypical PWR pressure, temperature, and heat flux. Target operating conditions are given in Table 3.17. The rods were 7 feet in length and uniformly electrically heated in the axial direction; rods were non-uniformly heated in the radial direction, as shown in the cross-section diagrams of the facility in Figures 3.16 and 3.17. The grids contained no mixing vanes. Thermocouples were placed at four axial locations in both the central heater tube and one adjacent heater tube. Additionally, pressure measurements were made via four pressure taps. Figure 3.18 shows a simple axial diagram of the assembly.

Pressure drop measurements were made at cold conditions (80 F and $130 \mathrm{~F}$ ) at a pressure of 1000 psia. The inlet mass velocity was varied such that Reynolds-dependant grid-loss correlations could be developed. The Moody friction factor correlation was used to characterize the frictional component of pressure drop. The grid loss coefficients were calculated by the authors on a persubchannel basis considering the blockage ratio and wetted perimeter of each subchannel (see Table 5.1 of specification[9]). These loss coefficients were used in the CTF model.

Heated tests were run at a wide range of heat fluxes in order to cause single-phase, subcooled boiling, and saturated boiling regions of the boiling curve in the upper assembly. Transition to boiling was detected by observing a "levelling-off" of the rod surface temperature with increasing heat flux. Additionally, several DNB tests were performed by ramping up the power in the facility until a sharp temperature increase was detected at the thermocouple locations, indicating a postCHF heat transfer regime has been entered. CHF results are presented in Table 3.18. Two of the tests are shown as experiencing $\mathrm{CHF}$ in two locations.

Table 3.17: Target operating conditions for CE 5x5 Tests 74 and 75

\begin{tabular}{rrr}
\hline Inlet Temperature (F) & Pressure (psia) & Mass Velocity $\left(\mathrm{Mlbm} / \mathrm{hr}^{-\mathrm{ft}^{2}}\right)$ \\
\hline 564 & 2250 & 2.25 \\
583 & 2250 & 2.25 \\
543 & 2250 & 2.97 \\
512 & 1750 & 2.25 \\
\hline
\end{tabular}




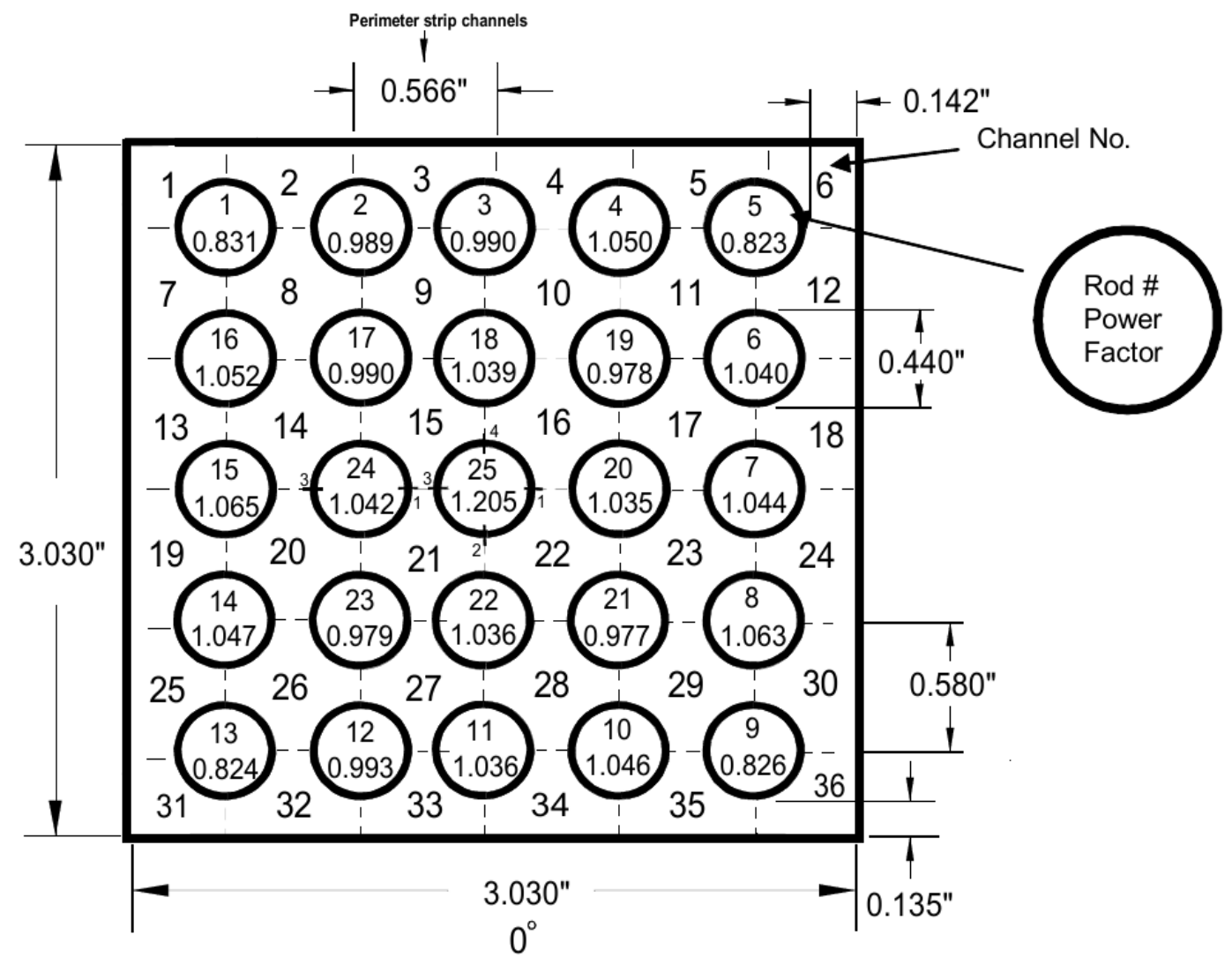

Figure 3.16: Cross-section diagram of CE $5 \times 5$ Test 74

Table 3.18: CHF results for CE 5x5 Tests 74 and 75

\begin{tabular}{|c|c|c|c|c|c|}
\hline Test & $\begin{array}{r}\text { Inlet Temperature } \\
(\mathrm{F})\end{array}$ & $\begin{array}{r}\text { Pressure } \\
(\text { psia })\end{array}$ & $\begin{array}{l}\text { Mass Velocity } \\
\left(\text { Mlbm/hr-ft }{ }^{2}\right)\end{array}$ & $\begin{array}{r}\text { Rod CHF } \\
\left(\mathrm{MBTU} / \mathrm{hr}-\mathrm{ft}^{2}\right)\end{array}$ & $\begin{array}{r}\mathrm{T} / \mathrm{C} \mathrm{CHF} \\
\text { Location (in) }\end{array}$ \\
\hline 74 & 562.5 & 2254.7 & 2.259 & 0.652 & 25.43 \\
\hline 74 & 545.7 & 2254.7 & 2.236 & 0.697 & 25.43 \\
\hline 74 & 514.0 & 1749.7 & 2.203 & 0.695 & $\begin{array}{l}25.43 \\
25.41\end{array}$ \\
\hline 74 & 582.0 & 2254.7 & 2.964 & 0.718 & 25.43 \\
\hline 75 & 563.5 & 2249.7 & 2.281 & 0.679 & 25.43 \\
\hline 75 & 547.5 & 2249.7 & 2.211 & 0.708 & 25.43 \\
\hline 75 & 516.0 & 1749.7 & 2.224 & 0.715 & $\begin{array}{l}25.43 \\
25.41\end{array}$ \\
\hline 75 & 584.0 & 2249.7 & 2.927 & 0.743 & 25.43 \\
\hline
\end{tabular}




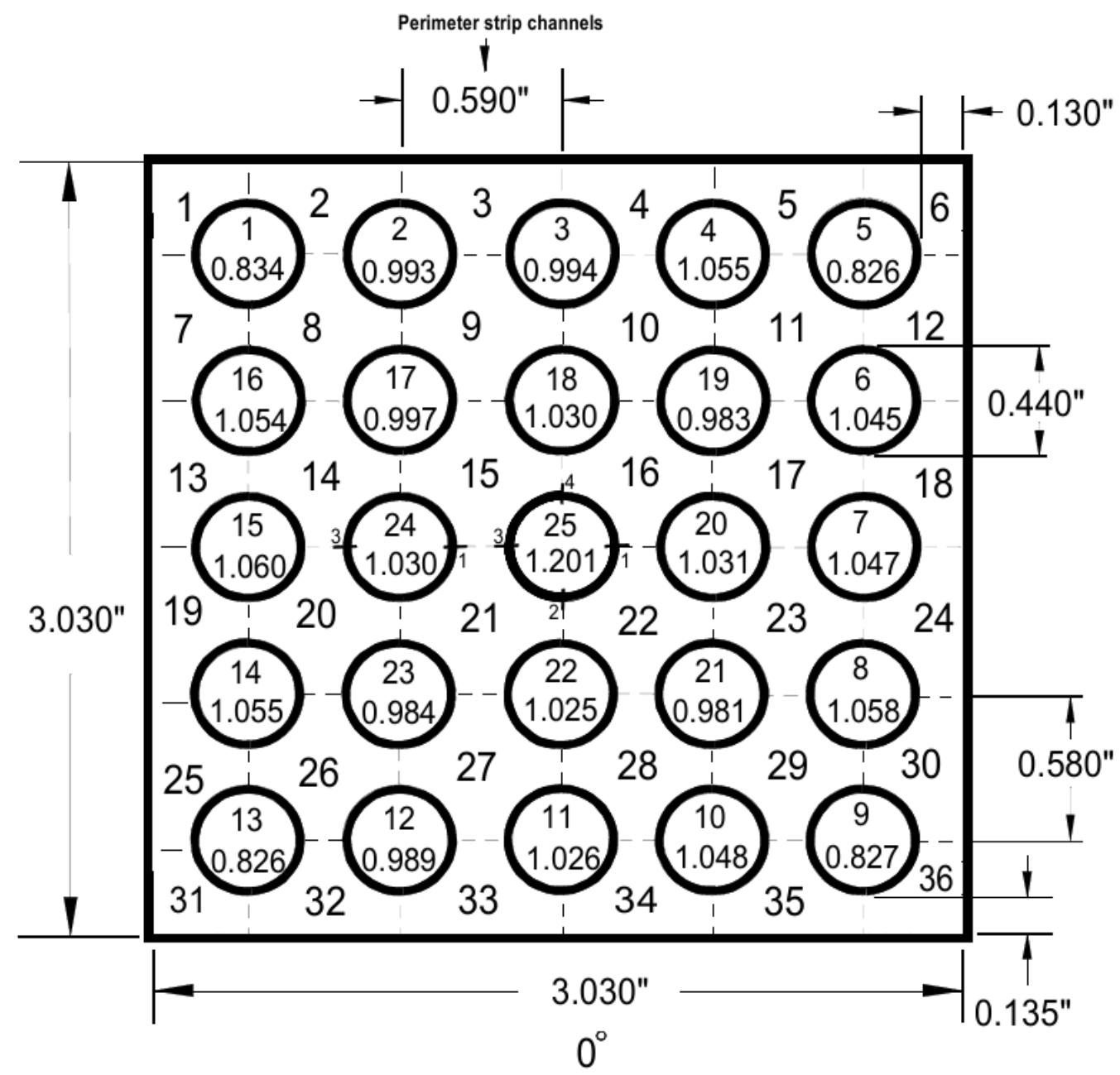

Figure 3.17: Cross-section diagram of CE $5 \times 5$ Test 75 


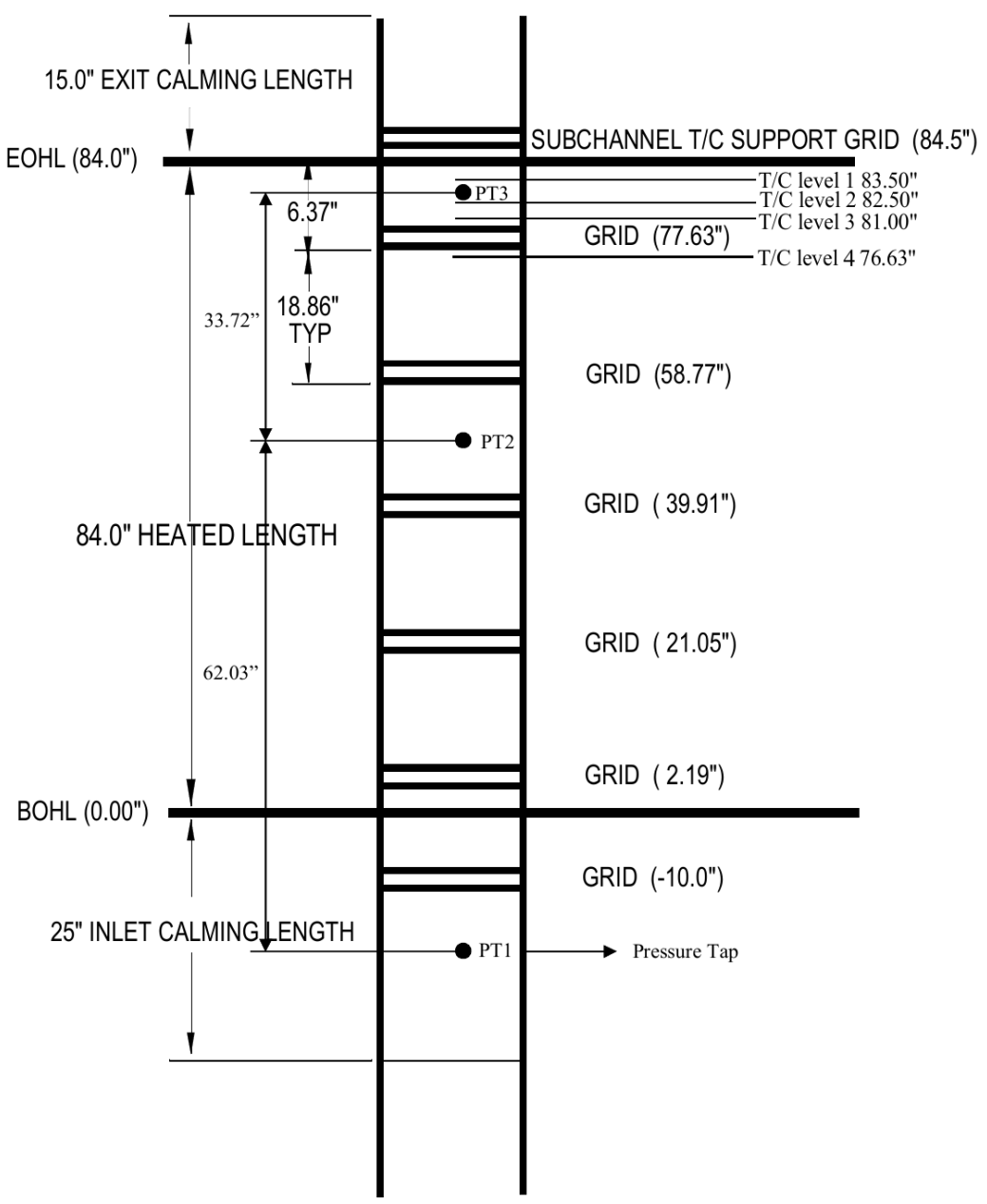

Figure 3.18: Axial schematic of the CE 5x5 bundle 


\subsection{GE $3 \times 3$}

The GE 3x3 facility is a classic test for assessing inter-subchanenl mixing[10] due to the fact that mass flux and quality measurements could be made for individual subchannel types. A $3 \times 3$ heated tube geometry was used in a BWR-like simulation with General Electric (GE) rods[11]. The geometry of the bundle is shown in Figure 3.19. The details of this bundle are provided in Table 3.19. A summary of the geometry of the 16 subchannels is provided in Table 3.20.

The axial and radial power profiles were uniform for all of these test cases. The same bundle was used for all the tests. Bundle power, flow rate, and inlet subcooling was varied between different experimental cases.

Subchannel measurements were taken to determine the specifics of coolant flow through different types of subchannels. The target parameters were the flow rate and the enthalpy as compared to the bundle-averaged values. The $2 \mathrm{~B}$ and $2 \mathrm{D}$ series test cases all utilize a uniform radial and axial heat flux distribution. The main difference between the two series is the power level. The subcooling is specified as well, as is shown in Table 3.21.

There are pins holding the rods in place which act as spacers. These pins are $3.18 \mathrm{~mm}$ in diameter and either $3.43 \mathrm{~mm}$ long or $4.27 \mathrm{~mm}$ long, for either rod-rod or rod-wall connections, respectively. There are four pins connected to each rod spaced evenly azimuthally and perpendicular to the shroud surface where they connect. These locations are along the subchannel boundaries which are indicated in Figure 3.20. Pins labeled with either 'A' or 'B' are $3.43 \mathrm{~mm}$ in length, while pins labeled with either ' $C$ ' or ' $\mathrm{D}$ ' are $4.27 \mathrm{~mm}$ in length. All pins have the same diameter: $3.18 \mathrm{~mm}$. These pins were fusion-welded to the outside of each pin and to the walls.

There are six sets of 24 pins spaced every $304.8 \mathrm{~mm}$ starting $50.8 \mathrm{~mm}$ from the bottom of the heated length. The locations of these six pin spacers are 50.8, 355.6, 660.4, 965.2, 1270.0, and $1574.8 \mathrm{~mm}$ from the bottom of the heated length.

Measured values from the experiments include:

- Normalized subchannel mass flux versus bundle-averaged quality

\begin{tabular}{lr}
\hline Item & Description \\
\hline Number of heater rods & 9 \\
Heater rod OD (mm) & 14.5 \\
Heated length $(\mathrm{mm})$ & 1828.8 \\
Rod pitch (mm) & 18.7 \\
Channel box inner width $(\mathrm{mm})$ & 58.83 \\
Channel box corner radius $(\mathrm{mm})$ & 10.2 \\
Flow area $\left(\mathrm{mm}^{2}\right)$ & 1890.3 \\
Number of spacers & 6 \\
Spacer type & Pin \\
\hline
\end{tabular}

Table 3.19: GE $3 \times 3$ Bundle Description 


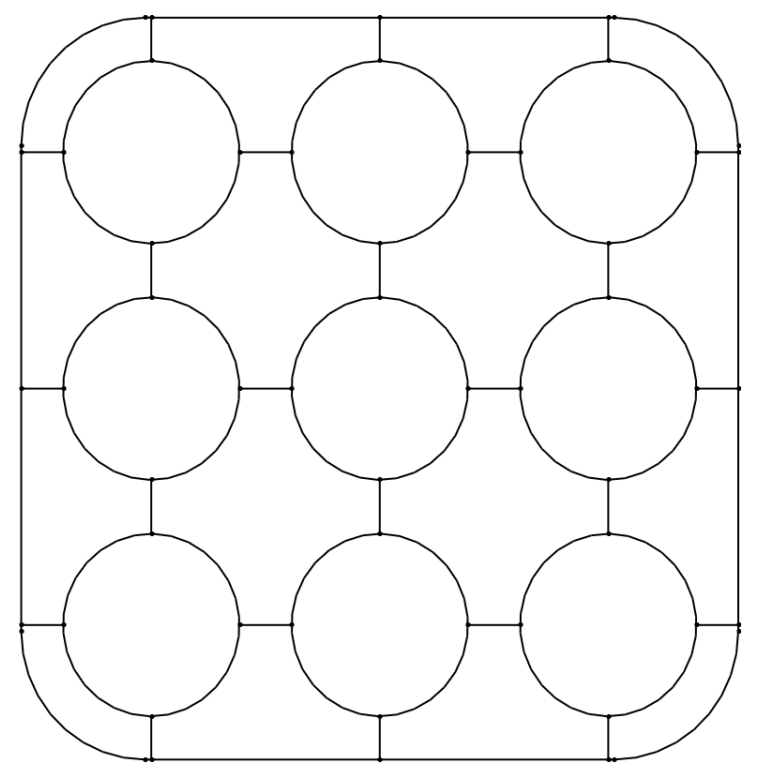

Figure 3.19: GE $3 \times 3$ Bundle Geometry

Table 3.20: GE $3 \times 3$ Bundle Subchannel Geometry

\begin{tabular}{rrr}
\hline Subchannel & $\begin{array}{r}\text { Area } \\
\mathrm{m}^{2}\end{array}$ & $\begin{array}{r}\text { Wetted Perimeter } \\
\mathrm{m}\end{array}$ \\
\hline Corner & $5.050 \mathrm{E}-05$ & $2.835 \mathrm{E}-02$ \\
Side & $1.177 \mathrm{E}-04$ & $4.149 \mathrm{E}-02$ \\
Center & $1.868 \mathrm{E}-04$ & $4.548 \mathrm{E}-02$ \\
\hline
\end{tabular}


Table 3.21: GE $3 \times 3$ Bundle Boundary Conditions by Test Series

\begin{tabular}{rrrr}
\hline Test Series & $\begin{array}{r}\text { Power } \\
{[\mathrm{kW}]}\end{array}$ & $\begin{array}{r}\dot{m}_{\text {in }} \\
{[\mathrm{kg} / \mathrm{s}]}\end{array}$ & $\begin{array}{r}\text { Inlet Subcooling } \\
{[\mathrm{kJ} / \mathrm{kg}]}\end{array}$ \\
\hline $1 \mathrm{~B}$ & 0 & 1.231 & 504.6 \\
$1 \mathrm{C}$ & 0 & 2.538 & 504.6 \\
$1 \mathrm{D}$ & 0 & 3.871 & 504.6 \\
$1 \mathrm{E}$ & 0 & 5.054 & 504.6 \\
$2 \mathrm{~B} 2$ & 532 & 1.360 & 348.4 \\
$2 \mathrm{~B} 3$ & 532 & 1.373 & 252.6 \\
2B4 & 532 & 1.373 & 122.7 \\
$2 \mathrm{C} 1$ & 532 & 2.720 & 132.9 \\
$2 \mathrm{C} 2$ & 532 & 2.740 & 81.57 \\
$2 \mathrm{D} 1$ & 1064 & 1.386 & 602.4 \\
$2 \mathrm{D} 3$ & 1064 & 1.386 & 289.1 \\
2E1 & 1064 & 2.771 & 332.1 \\
$2 \mathrm{E} 2$ & 1064 & 2.771 & 224.7 \\
$2 \mathrm{E} 3$ & 1064 & 2.712 & 67.63 \\
$2 \mathrm{E} 1$ & 1064 & 2.771 & 332.1 \\
$2 \mathrm{E} 2$ & 1064 & 2.771 & 224.7 \\
$2 \mathrm{E} 3$ & 1064 & 2.712 & 67.63 \\
$2 \mathrm{G} 1$ & 1596 & 2.746 & 525.0 \\
$2 \mathrm{G} 2$ & 1596 & 2.771 & 441.1 \\
$2 \mathrm{G} 3$ & 1596 & 2.746 & 340.9 \\
\hline & & &
\end{tabular}




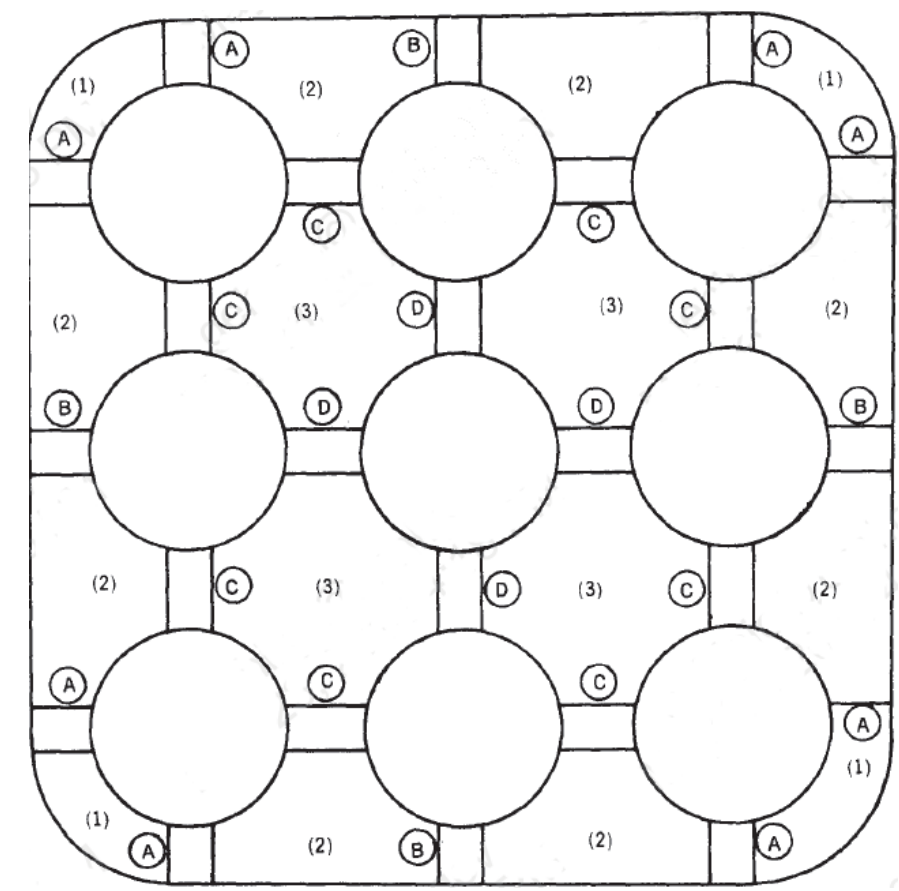

Figure 3.20: GE $3 \times 3$ Bundle Pin Spacer Geometry

- Normalized subchahnel enthalpy versus bundle-averaged quality

The measurements taken during the experiment were mass flows and enthalpies of specific subchannels. These were grouped by subchannel type: corner, side, or center. The subchannels were isolated using special ducting in order to obtain measurements at only the subchannel type of interest. Once isolated using sheets of metal, the flow rate and enthalpy of the fluid passing through the subchannel are measured using a turbine flow meter (for the flow) and by a heat balance on a calorimeter (for the enthalpy). Pressure loss values were also measured using pressure transducers across the entire bundle [11].

The normalized subchannel mass flux and enthalpy is calculated for each of the three types of subchannels in this bundle. They are normalized in order to gain a comparison of that particular subchannel's flow or enthalpy to that of the entire bundle as a whole. The flow normalization is performed by Equation (3.1). Similarly, the enthalpy normalization is performed as shown in Equation (3.2).

$$
\begin{gathered}
\frac{G_{c h}-\bar{G}}{\bar{G}} \\
\frac{H_{c h}-\bar{H}}{\bar{H}}
\end{gathered}
$$

In these equations, $\bar{G}$ and $\bar{H}$ correspond to the bundle-averaged values of flow rate and 
enthalpy, respectively, and $\mathrm{G}_{c h}$ and $\mathrm{H}_{c h}$ are the flow rate and enthalpy of the subchannel type.

\subsection{Harwell High Pressure Loop}

Post-dryout (post-CHF) experiments were performed in the Harwell High Pressure Two-Phase Heat Transfer Loop facility[12]. The facility consisted of an electrically heated tube with water flowing in the inside. The tube had a 0.496 inch inner diameter, was $19 \mathrm{ft}$ long, and was vertically aligned. Thermocouples were affixed to the outside of the tube, the spacing being as large as 12 inches and as small as 3 inches near the top of the test section. A series of tests were performed where the flow conditions were such that dryout occurred at some location in the tube. This was achieved by adjusting inlet flow rate and test section power; the outlet pressure was fixed at 1000 psi for all tests.

The testing procedure involved increasing the power until a sharp temperature increase was detected, indicating that dryout had occurred. Measured temperatures were used to calculate the tube inside surface temperatures, which were summarized for all tests in the report by Bennett, et al.[12].

Two varieties of tests were performed; one with a 144 inch active length and another with a 219 inch active length. Only the 219 inch active length tests were modeled in CTF. Therefore, the geometry and problem mesh were the same for all tests modeled. Only the flow conditions were varied from test-to-test. A schematic of the CTF axial mesh is shown in Figure 3.21. The CTF model consisted of one channel and one heater tube. The model was setup as if the flow was on the exterior of the heater tube. The outer diameter of this tube was set equal to the inner tube diameter so that the tube surface area of the rod/fluid interface was consistent with the experiments. The channel flow area and wetted perimeter in the CTF subchannel was set equal to the flow area of the inside of the tube. Only the active region of the experiment was modeled. The axial meshing was done such that the center of the CTF mesh cells were within 4 $\mathrm{mm}$ of the actual measurement location in the experiment.

The axial mesh is laid out in groups of cells all having the same height. In the figure, the green block on the bottom denotes a group of cells having 4 inch heights. From 112 inches and up, the mesh is gradually refined. The blue block represents a region where the mesh size is 0.5 inches. This was done because this region (112 inches and up) is where dryout occurs, which requires a finer mesh. With the way the mesh was setup, the axial locations of test facility thermocouples always line up with a CTF cell center.

Due to the inclusion of the droplet field entrainment/de-entrainment and the complex physics being modeled, it was difficult to converge these tests to a level of tolerance commensurate with that of simpler, single-phase tests. Therefore, the minimum and maximum allowable timestep sizes were set to $1 \mathrm{E}-7 \mathrm{~s}$ and $1 \mathrm{E}-4 \mathrm{~s}$, respectively and the ratio between the conduction and fluid solution time scales was set to 1.0 (a value we normally increase to 10,000). Even with these extra measures, it was typically only possible to drop the mass and energy storage terms to about $0.1-1 \%$. Mass and energy balance terms were generally more well behaved, dropping to $0.001-0.01 \%$.

Boundary conditions were set to match experimental values; a pressure boundary condition was set at the test outlet and a mass flow rate and enthalpy boundary condition was set at 


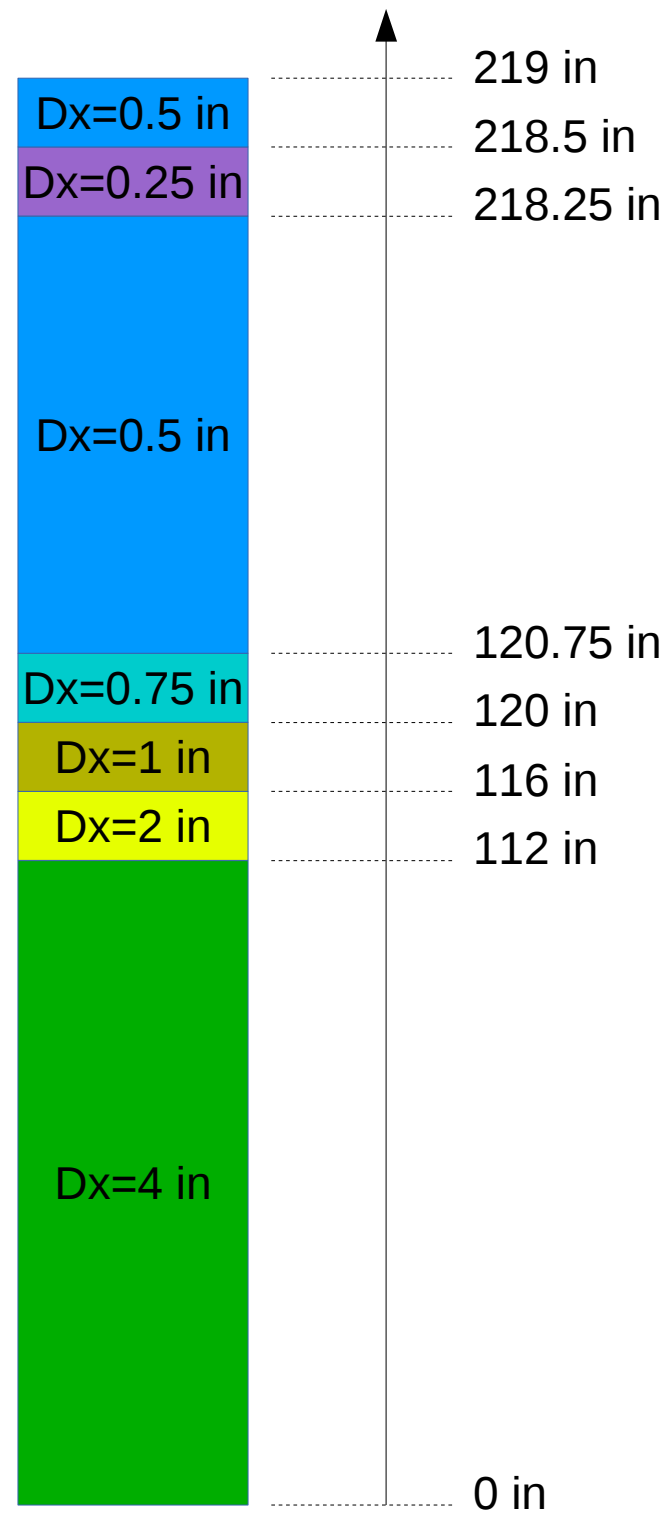

Figure 3.21: CTF model of the Harwell High-Pressure Two-Phase Test Facility 
the inlet. The author provided inlet subcooling as a boundary condition, so the tests were run in an iterative fashion to match the inlet subcooling to experimental values. The tube material properties were set to that of Zircaloy, though this has no impact on the solution value of interest, the tube surface temperature, because we run the case to steady-state, where all heat generated in the model is being deposited into the fluid.

\subsection{FRIGG}

The FRIGG test facility consists of an electrically heated rod bundle, modeled after the Marviken reactor assemblies[13]. Experiments were run in the facility to verify that the Marviken reactor could be run at the rated power with proper safety margins against burnout. Investigations included single- and two-phase pressure drop measurements, axial and radial void distribution measurements, and burnout in natural and forced circulation. In this study, we model a single uniformly heated, natural convection, two-phase flow test case from the study.

\subsubsection{CTF Model of Facility}

\subsubsection{Radial Meshing}

The Marviken reactor uses fuel assemblies having lattices much different than that of a typical U.S. PWR. The 36 fuel rods are situated so that their centers lie on rings of increasing size going outwards from the center of the circular shaped bundle. An unheated guide tube lies at the center of the bundle. The first ring of rods occurs at $21.6 \mathrm{~mm}$ from the center and contains 6 fuel rods. The second ring of rods occurs at $41.7 \mathrm{~mm}$ from center and contains 12 fuel rods. The third and final ring occurs at $62.2 \mathrm{~mm}$ from center and contains 18 rods. This leads to a total of 36 fuel rods and one central guide tube. The canister for the fuel assembly has a diameter of $159.5 \mathrm{~mm}$. The gap between rods on the same ring is $7.8 \mathrm{~mm}$.

Figure 3.22 shows the cross-section of the Marviken assembly (and the FRIGG test facility). The CTF modeling approach for this facility is also shown in the figure. To simplify the modeling of the facility, a lumped-subchannel approach was utilized. The space between the central guide tube and the first ring of rods formed the first subchannel in the CTF model. Likewise, the space between the first and second ring of rods formed the second subchannel, and so on. In addition to lumping the subchannels together, it also made sense to lump fuel rods and gaps, as well. The first ring of rods were lumped into Rod 2, the second ring of rods were lumped into Rod 3 , and the third ring of rods were lumped into Rod 4. In this way, there were no gaps modeled in the azimuthal $(\theta)$ direction of the circle; rather, gaps only exist in the radial $(r)$ direction. Since the rod spacing is always $7.8 \mathrm{~mm}$ in the $\theta$ direction, it was possible to lump all gaps on a given ring together.

To create the CTF model, it was necessary to calculate the cross-flow area of the rings as well as their wetted perimeter. Any given fuel rod resides in exactly two rings. In order to obtain the channel area and wetted perimeter, it was necessary to determine how much of the fuel rod is in each of the two rings it occupies. To do this, we first zoom in on a single fuel rod and define an equation for the circle whose circumference passes through the fuel rod center. This is shown 


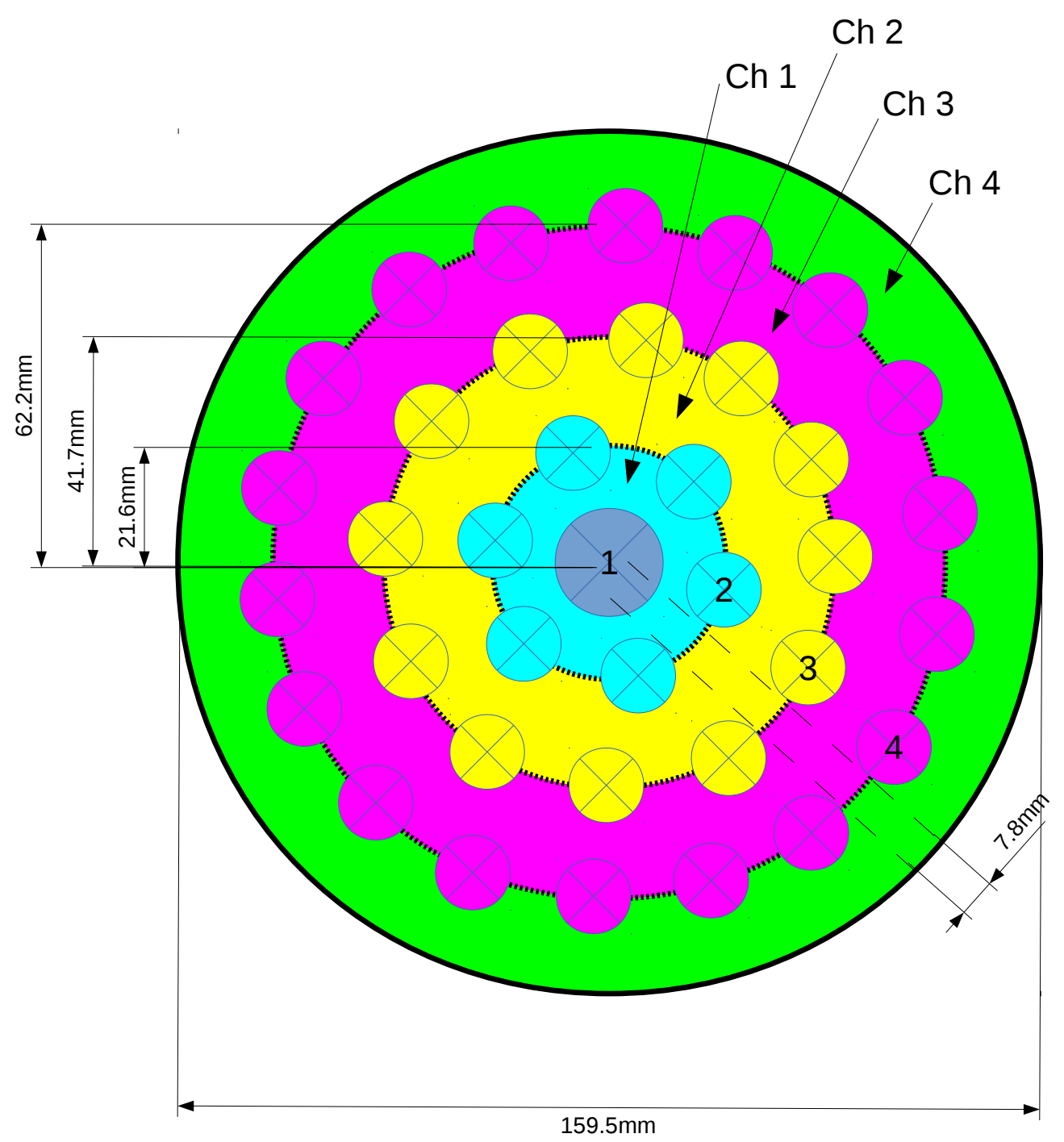

Figure 3.22: Cross-section of the FRIGG facility (including labels of CTF model rods and channels) 


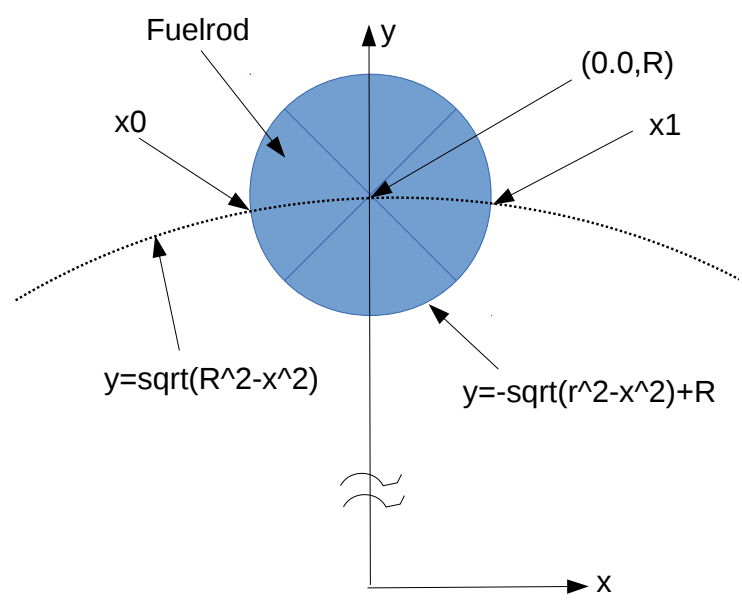

Figure 3.23: Definition of functions for the circle that defines the fuel rod and the circle it sits on

in Figure 3.23, where the blue circle represents the fuel rod. If we assume that the large circle's center is at the origin, we can define functions for both circles.

To obtain the area of the fuel rod in the smaller of the two rings, we can simply integrate the difference of the two functions that define the larger and smaller circles between their intersections. In equation form:

$$
A_{\text {small }}=\int_{x_{0}}^{x_{1}} f_{\text {large circle }}(x)-f_{\text {small circle }}(x)
$$

Here, $A_{\text {small }}$ is the cross sectional area of the rod residing in the smaller channel. Substituting the actual equations of the circles leads to the following:

$$
A_{\text {small }}=\int_{x_{0}}^{x_{1}} \sqrt{R^{2}-x^{2}}-\left(-\sqrt{r^{2}-x^{2}}+R\right)
$$

Here, $R$ is the radius of the large circle and $r$ is the radius of the small circle that represents the fuel rod. It was assumed that the fuel-rod circle lay directly above the center of the large circle, so that its equation is simply shifted up the $y$ axis and requires no shift in the $x$ direction. Since the fuel-rod circle center is situated on the larger circle circumference, the fuel-rod circle equation is shifted up the y-axis by distance $R$. The intersection points of the two circles can be found by setting the equations equal to one another and solving for $x$. Since the fuel-rod circle is directly above the large circle center, the two intersection points have the same magnitude, but opposite sign: 
Table 3.22: Channel area displaced by each rod bank

\begin{tabular}{rrrrrr}
\hline \multicolumn{6}{c}{ Displacement of Channel Area $\left(\mathrm{mm}^{2}\right)$} \\
Rod Bank & Ch 1 & Ch 2 & Ch 3 & Ch 4 & Num Rods \\
\hline 1 & 314.16 & 0.00 & 0.00 & 0.00 & 1 \\
2 & 69.70 & 79.61 & 0.00 & 0.00 & 6 \\
3 & 0.00 & 72.22 & 77.38 & 0.00 & 12 \\
4 & 0.00 & 0.00 & 73.03 & 76.54 & 18 \\
\hline
\end{tabular}

$$
\begin{gathered}
x_{0}=-\sqrt{r^{2}-\frac{r^{2}}{4 R^{2}}} \\
x_{1}=\sqrt{r^{2}-\frac{r^{2}}{4 R^{2}}}
\end{gathered}
$$

Doing the integration of Equation 3.4 from the bounds given in Equations 3.5 and 3.6 yields the following equation for cross-sectional area of a rod residing in the smaller channel:

$$
A_{\text {small }}=\left[\frac{x}{2} \sqrt{R^{2}-x^{2}}+\frac{R^{2}}{2} \sin ^{-1} \frac{x}{R}+\frac{x}{2} \sqrt{r^{2}-x^{2}}+\frac{r^{2}}{2} \sin ^{-1} \frac{x}{r}-R x\right]_{x_{0}}^{x_{1}}
$$

The area of the rod residing in the larger channel is then simply calculated as the total rod cross-sectional area minus the smaller area given by Equation 3.7.

$$
A_{\text {large }}=\pi r^{2}-A_{\text {small }}
$$

The channel cross-sectional area that is displaced by the rods are given in Table 3.22. Note that this table gives displacement for a single rod in the bank, so the total displacement area of the entire bank must be calculated by multiplying the number of rods in the bank by the displacement of the single rod.

As a sanity check, it is prudent to compare the sum of the values in Table 3.22 times number of rods in the bank to a simple calculation of the total rod cross-sectional area in the test section. The rod cross-section area can be calculated simply as follows:

$$
A=\frac{\pi}{4} 20^{2}+36 \frac{\pi}{4} 13.8^{2}=5698.7 \mathrm{~mm}^{2}
$$

Doing the math on Table 3.22 results in a total displacement area of $5697.48 \mathrm{~mm}^{2}$, which agrees with our sanity check to within $0.021 \%$, which can easily be accounted for by roundoff.

To figure out the wetted perimeter, it's necessary to find the angle between the two intersection points, $x_{0}$ and $x_{1}$, and the center of the fuel-rod circle. The trigonometric relation for determining this angle is presented below: 
Table 3.23: Wetted perimeter of each rod in each channel of the model

\begin{tabular}{rrrrrr}
\hline \multicolumn{6}{c}{ Rod Wetted Perimeter $(\mathrm{mm})$} \\
Rod Bank & Ch 1 & Ch 2 & Ch 3 & Ch 4 & Num Rods \\
\hline 1 & 62.83 & 0.00 & 0.00 & 0.00 & 1 \\
2 & 19.46 & 23.89 & 0.00 & 0.00 & 6 \\
3 & 0.00 & 20.53 & 22.82 & 0.00 & 12 \\
4 & 0.00 & 0.00 & 20.91 & 22.44 & 18 \\
\hline
\end{tabular}

Table 3.24: Azimuthal fraction of each rod in each channel of the model

\begin{tabular}{rrrrr}
\hline & \multicolumn{4}{c}{ Azimuthal fraction } \\
Rod Bank & Ch 1 & Ch 2 & Ch 3 & Ch 4 \\
\hline 1 & 1.0000 & 0.0000 & 0.0000 & 0.0000 \\
2 & 0.4489 & 0.5511 & 0.0000 & 0.0000 \\
3 & 0.0000 & 0.4736 & 0.5264 & 0.0000 \\
4 & 0.0000 & 0.0000 & 0.4823 & 0.5177 \\
\hline
\end{tabular}

$$
\theta=\pi-2 \cos ^{-1} \frac{x_{0}}{r}
$$

It's then possible to calculate the arc length between the two points with the relationship $\pi \theta$. The wetted perimeter of each rod in each ring is shown in Table 3.23. Similar to Table 3.22, the table gives wetted perimeter for a single rod in the bank (not the entire bank).

As a sanity check on the wetted perimeters, the total wetted perimeter of all rods is compared to the sum of the values given in Table 3.23 multiplied by the number of rods in each bank:

$$
P_{w}=\pi 20+36 \pi 13.8=1623.6 \mathrm{~mm}
$$

This compares favorable with the total displacement calculated from Table 3.23, which is $1623.43 \mathrm{~mm}$ (a $0.010 \%$ difference). Note that in the CTF model, the wetted perimeter of the assembly casing is also added to Channel 4 .

It is necessary to determine the azimuthal fraction of each rod bank in the channel so that rod heat is properly distributed into the channels. This can be obtained by taking the ratio of rod perimeter in the channel (given in Table 3.23) to total rod perimeter (43.4 mm). Azimuthal fractions of rods in given channels are shown in Table 3.24.

Finally, the gap length and width must be determined to create the 3 gaps that exist between the 4 channels. The width is simply $7.8 \mathrm{~mm}$, which was given in the facility schematic. The length is calculated as the distance between ring centers:

$$
L=\frac{R_{i}-R_{i-1}}{2}+\frac{R_{i+1}-R_{i}}{2}
$$




\subsubsection{Axial Meshing}

A schematic of the assembly in the axial direction is given in Figure 3.24. The figure shows the location of pressure taps with a "P" (e.g. P10, P11), thermocouples with a "T", and gamma densitometers (void measurements) with a "G". Spacer grid locations are labeled "Spacer" in the figure. The CTF model was made to cover the test section from the beginning of heated length (labeled as 0 in the figure) to the location of the P20 pressure tap, located at 4781 $\mathrm{mm}$. As indicated in the drawing, the heated length is $4378 \mathrm{~mm}$. The mesh cell sizing was meticulously set so that scalar cell centers lined up perfectly with the location of a pressure tap, thermocouple measurement, or gamma densitometer measurement. The scalar cell boundaries lined up perfectly with the location of spacer grids (which are placed at the center of momentum mesh cells in CTF). This ensured we could do direct CTF-to-experimental comparisons after running the simulation. A total of 123 mesh cells were used, leading to an average mesh cell height of $38.9 \mathrm{~mm}$.

\subsubsection{Boundary Conditions}

Results were to be compared to those presented in Figure 26 of the test report[13], so the boundary conditions of the case presented in that figure were used. The boundary conditions were given in the table in Appendix 1 of the report. The inlet mass flux was $1026 \mathrm{~kg} / \mathrm{m}^{2} \mathrm{~s}$, the outlet pressure was set to $50 \mathrm{bar}$, and the inlet enthalpy was set to $1122.5 \mathrm{~kJ} / \mathrm{kg}$ in order to achieve the specified inlet subcooling of $4.4{ }^{\circ} \mathrm{C}$. The total test section power was $3000 \mathrm{~kW}$, distributed uniformly throughout the test section. This test was a natural convection case.

\subsubsection{Modeling Choices}

Other important modeling choices included the selection of the grid loss coefficients and turbulentmixing/void-drift parameters. Page 26 of the report specifies that the mean spacer grid loss coefficient was 0.58 for a Reynolds number of $2 \times 10^{5}$, so this value was used in the CTF model. The report also mentioned that the friction factor correlation for the test section was $f=0.2 R e^{-0.2}$. The friction correlation used in CTF is $f=0.204 R e^{-0.2}$, so it matches the one specified in the experimental documentation well. The mixing model was set to use Rogers\&Rosehart to determine the single-phase mixing coefficient and Beus to determine the two-phase multiplier. The equilibrium void distribution weighting factor was set to the suggested value of 1.4 and the $\theta_{M}$ value was set to 5.0 . 


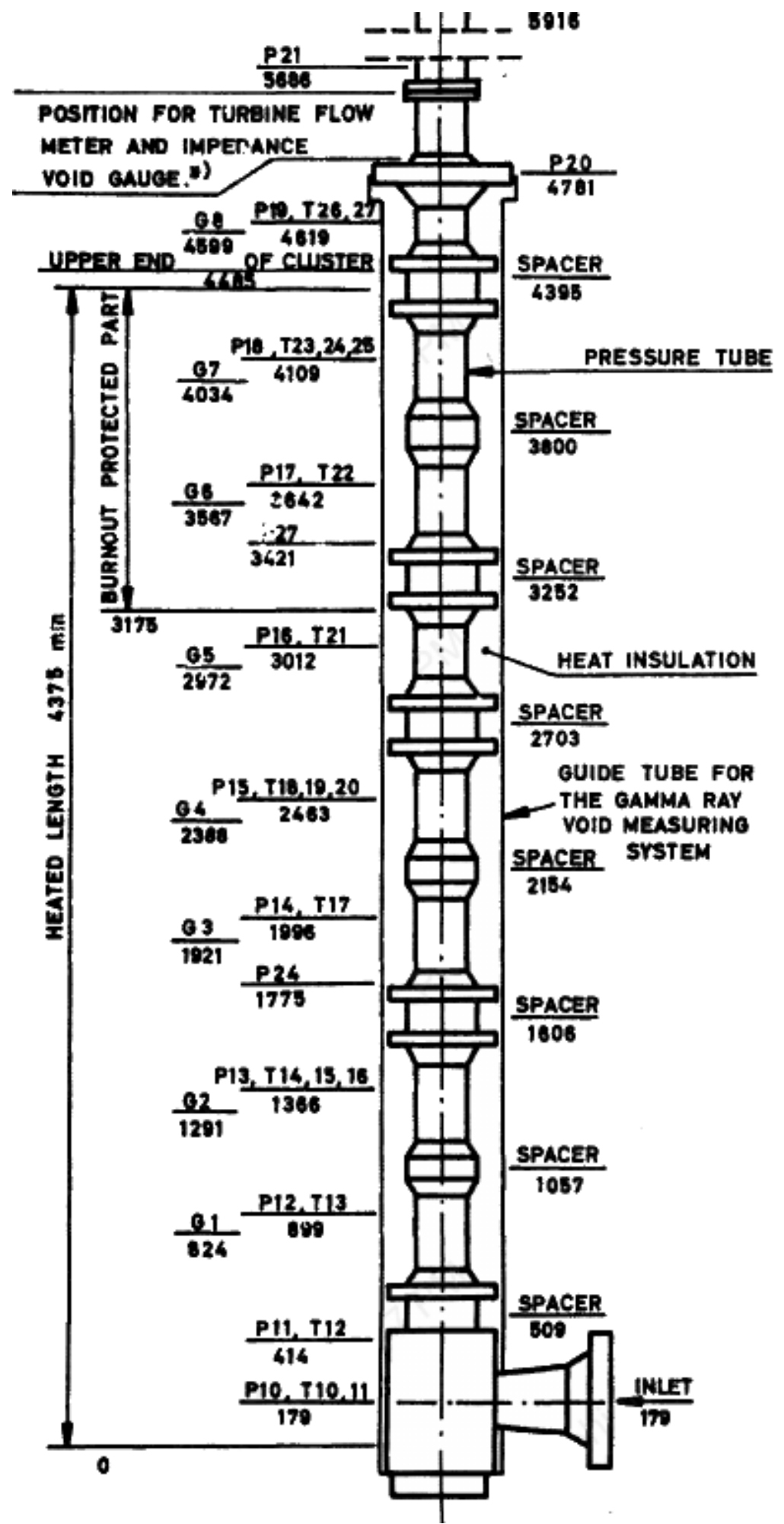

Figure 3.24: Schematic of FRIGG facility in axial direction 


\subsection{RPI $2 \times 2$}

The intended purpose of this experiment was to investigate the fully developed two-phase flow distribution in a $2 \times 2$ rod array test section. The test facility includes a 36 inch long unheated $2 \times 2$ rod bundle with an air-water mixture as the working fluid. With a bundle hydraulic diameter of 0.914 inches, an L/D factor of 39 was calculated, leading to an expected fully-developed flow condition at the bundle outlet. Four 0.055 inch thick 314 stainless steel tubes with 1.0 inch OD were used to simulate the fuel rods. The wall thickness insured a vibration-free environment during the experiment, and a lower tie plate provided support for the rods. No spacer grids were used in this experiment. Two different techniques were used to distribute the air into the bundle inlet, a sinter sections technique and a mixing tee technique [14].

Uncertainties within this experiment were measured with the theory of propagation of errors, which is based on the assumption of independent errors. The three independent variables in the error analysis were the subchannel liquid flow rate $\left(Q_{l i}\right)$, air flow rate $\left(Q_{g i}\right)$, and inlet pressure $\left(P_{i n}\right)$. Uncertainty intervals for each of these three variables were evaluated and used to derive the void fraction error interval, which was calculated for each data point using Equation 3.13. Using this expression, the errors in the subchannel void fractions were found to be no greater than $\pm 6.0 \%$.

$$
\Delta \alpha_{i}= \pm \frac{\left[2.1 \times 10^{-6} Q_{g i}^{2}+\left(1.16 \times 10^{-4} Q_{l i}+0.075 A_{i}\right)^{2}\right]^{\frac{1}{2}}}{\left\{0.343 Q_{g i}+0.046 Q_{l i}+29.02 A_{i}\right\}^{2}}
$$

The sinter section technique involved feeding the air at the upper tie plate, which was located at the bundle outlet, through the interior of the rods to sinter sections at the base of each rod. Each rod had its own separately controlled sinter section so that any number of the four rods could be supplied with air to simulate boiling. The cross sectional schematic of the sinter section can be found in Figure 3.25.

The mixing tee technique involved soldering thin slabs of sintered metal over cutouts in the water supply line for the test section. An air annulus was constructed to surround the porous section of the pipe, which allowed the air to be bubbled into the mainline. The cross sectional schematic of the mixing tee setup can be found in Figure 3.26.

In order to measure the void in each subchannel at the bundle outlet, one of each type of subchannel (corner, side, and center) were physically separated by means of 0.020 inch thick splitters located two inches from the test section outlet. These isolated subchannels were then simultaneously isokinetically sampled to determine the subchannel flow qualities according to Equation 3.14, where $G_{g}$ and $G_{l}$ refer to the water and air mass fluxes, respectively.

$$
\langle\bar{x}\rangle=\frac{G_{g}}{G_{l}+G_{g}}
$$

The subchannel void fraction and quality are related by the Zuber-Findlay equation as found in Equation 3.15, where $\rho_{g}$ and $\rho_{l}, V_{g j}$, and $C_{0}$ refer to the air and water densities, drift velocity, and void concentration parameter, respectively. 


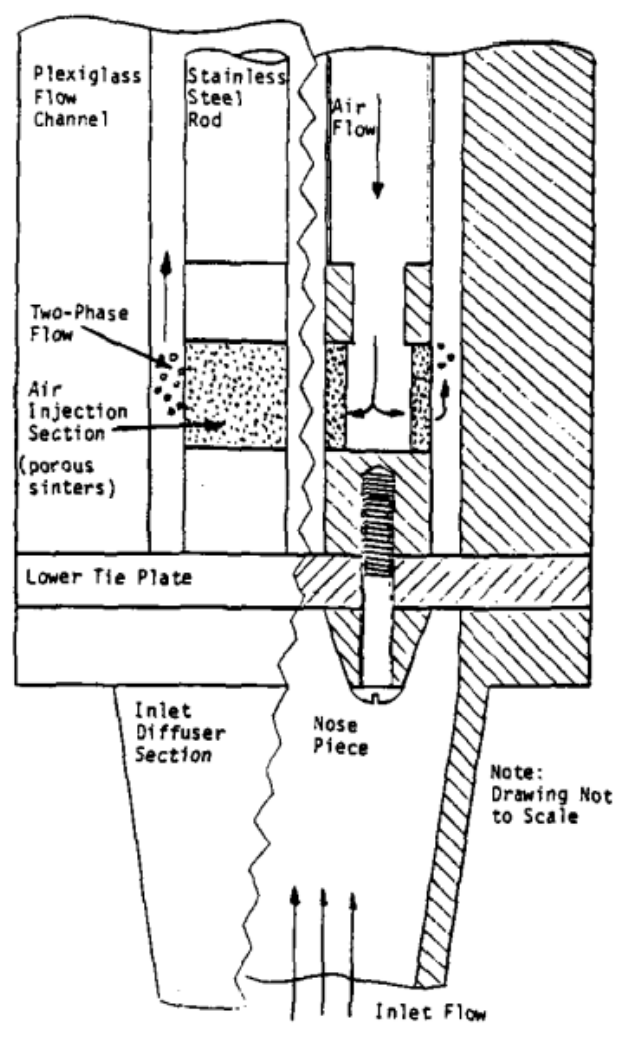

Figure 3.25: Low Assembly Test Section with Sinter Section Shown [14]

$$
\langle\alpha\rangle=\frac{\langle x\rangle}{\left\{C_{0}\left[\langle x\rangle+\frac{\rho_{g}}{\rho_{l}}(1-\langle x\rangle)\right]+\frac{\rho_{g} V_{g j}}{G}\right\}}
$$

The to-scale cross-sectional drawing of the test bundle can be found in Figure 3.27. The dimensions of this test section are twice that of a typical square pitched BWR fuel rod bundle in order to partially accommodate for the large bubble sizes present with the low pressure air-water experiment. The geometric dimensions used for each subchannel in the experiment and CTF simulations are found in Table 3.25. The different flow area values for these three subchannel types suggest that a below average void fraction would be measured in the corner and an above average void fraction would be measured in the center in the presence of a void drift mechanism. Subchannels 1, 2, and 3 were chosen to be isokinetically sampled to obtain void distribution data for the corner, side, and center subchannel types, respectively.

The operating conditions for these experiments can be found in Table 3.26, which include the water mass flux $(\mathrm{G})$, bundle averaged void fraction $(\langle\bar{\alpha}\rangle)$, and inlet pressure $\left(P_{\text {in }}\right)$. The fluid for all of the experiments was at ambient temperature. Besides the aforementioned four rod experiments, there were also a set of single phase experiments and two-phase single rod experiments described in the report. The two-phase single rod experiments were conducted to 


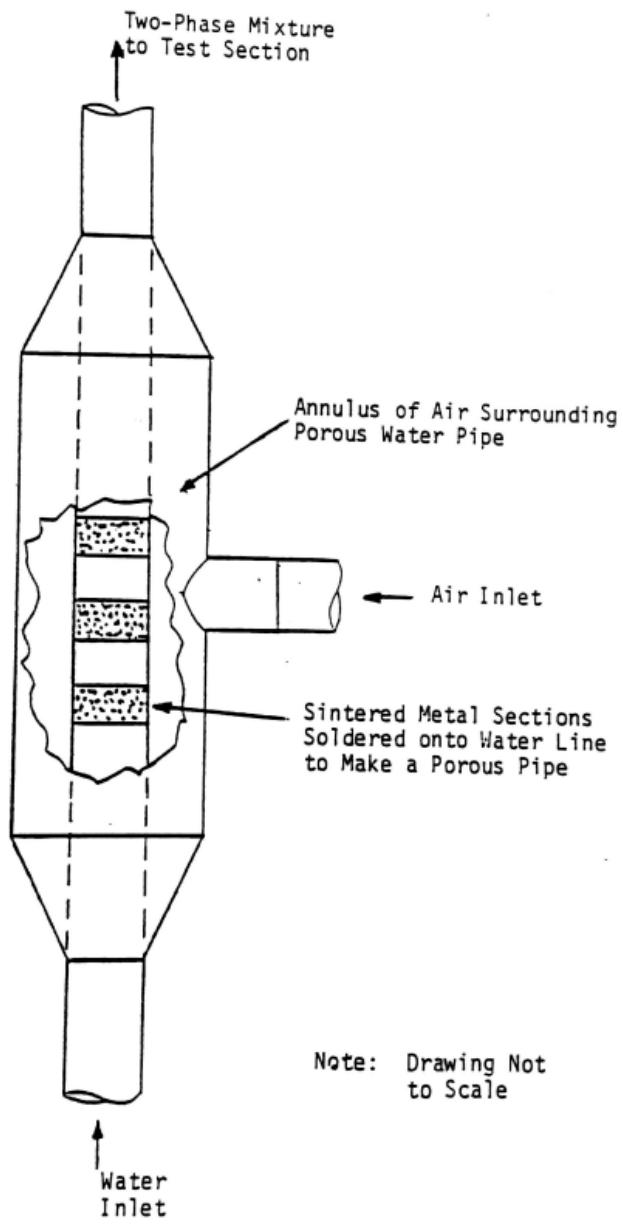

Figure 3.26: Air/Water Mixing Tee [14]

Table 3.25: NUREG Subchannel Flow Dimensions [14]

\begin{tabular}{rrr}
\hline Type & Flow Area $\left(\mathrm{in}^{2}\right)$ & Hydraulic Diameter (in) \\
\hline Corner & 2.08 & 1.59 \\
Side & 4.68 & 2.50 \\
Center & 7.13 & 3.57 \\
Total & 34.14 & 2.32 \\
\hline
\end{tabular}

observe the void drift phenomenon while only using the sinter section of one rod. 


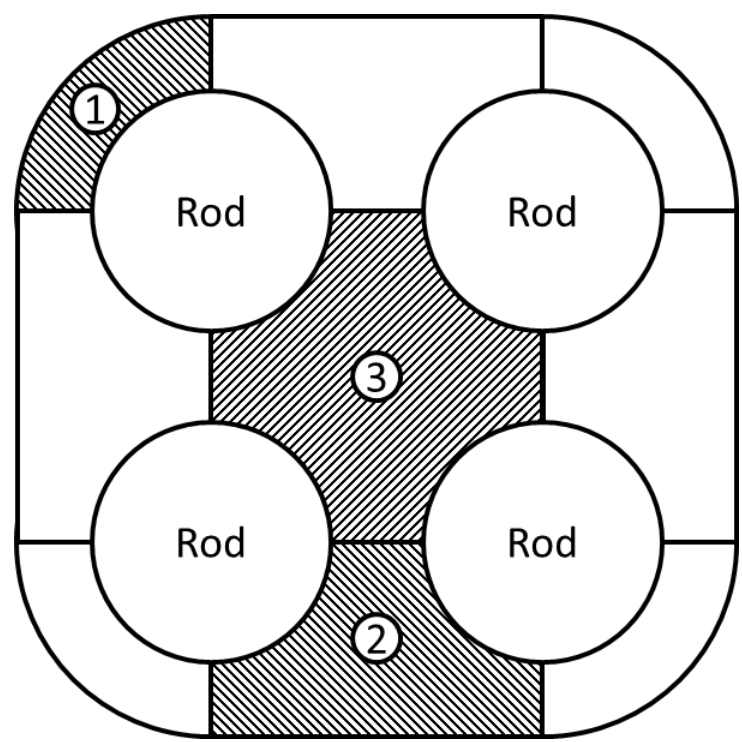

Figure 3.27: NUREG/CR-3373 Experimental Cross Section [14]

Table 3.26: Experimental Operating Conditions [14]

\begin{tabular}{rrrrr}
\hline Case & $G \times 10^{-6}\left[\frac{l b m}{f t^{2} h r}\right]$ & $\langle\bar{\alpha}\rangle[\%]$ & $P_{\text {in }}[$ psig $]$ & Mixing Technique \\
\hline 1 & 0.333 & 0.0 & 6.0 & \\
2 & 0.666 & 0.0 & 15.0 & \\
3 & 0.333 & 20.4 & 6.0 & \\
4 & 0.333 & 32.8 & 6.5 & \\
5 & 0.333 & 46.1 & 8.0 & Mixing Tee \\
6 & 0.666 & 23.6 & 15.5 & \\
7 & 0.666 & 37.5 & 19.0 & \\
8 & 0.666 & 52.7 & 26.0 & \\
\hline 9 & 0.333 & 20.4 & 6.0 & \\
10 & 0.333 & 32.8 & 6.3 & \\
11 & 0.333 & 46.1 & 8.0 & \multirow{2}{*}{4 Sinter Sections } \\
12 & 0.666 & 23.6 & 15.5 & \\
13 & 0.666 & 37.5 & 19.0 & \\
14 & 0.666 & 52.7 & 29.5 & \\
\hline
\end{tabular}




\subsection{Kumamoto Univeristy $2 \times 3$}

Facility description The $2 \times 3$ facility is an air/water facility that was operated at Kumamoto University in the early 2000s in order to quantify effects of mixing and void drift [15]. The test section is uniquely designed so that there are only two subchannel types - side and inner-leaving out the additional complexity of the corner type subchannel. Figure 3.28 shows the cross-section of the facility and provides geometric information. The assembly geometry is larger than a typical BWR lattice due to the lower density (and thus larger bubble sizes) of air at atmospheric pressure.

Figure 3.29 shows an axial schematic of the facility. The test section has four axial sections: 1) an entry section, where side and inner channels are separated by a physical barrier; 2) a tracer injection section, where each individual channel is physically separated from one another; 3) a mixing section, where all channel are connected and lateral cross-flow is allowed to take place; and 4) a discharge section, where groups of channels are partitioned and flow is sent to measurement devices.

Because the mixing section is short $(2.25 \mathrm{~m})$, the inlet flow of individual subchannel types is adjusted in the experiment so that flow in the mixing section is in mechanical equilibrium throughout the entire length. In this way, the test eliminates the effects of flow redistribution due to geometry differences between side and inner channels. The facility is used for two different types of tests: single- and two-phase mixing tests [15] and void drift tests [16].

The mixing tests made use of tracer dyes in the facility. The tracer dyes include Acid orange II for the water phase and methane for the gas phase. They are injected into one of the inner subchannels in the tracer injection section and collected and measured at three axial elevations in the mixing section. Measurements are taken by gas chromatograph for the gas phase and spectrophotometer for the liquid phase.

The author derived a tracer-dye conservation equation for each subchannel and used this system of equations to derive the channel mixing term, $w_{i j}^{\prime}$, as a function of tracer dye concentration. Flow conditions for the mixing tests are shown in Figure 3.30 as liquid and vapor superficial velocity. Only the single-phase tests are modeled in this study, which includes four data points. The system is run at room temperature and atmospheric pressure.

CTF Model Description Flow area and wetted perimeter are directly taken from the values of Figure 3.28. An axial mesh size of $2.54 \mathrm{~cm}$ is employed, and only the $2.25 \mathrm{~m}$ mixing section is modeled. Because the experimenters set the inlet flow to equal the equilibrium distribution, a similar approach is used in setting the inlet flow rate in CTF. First, the total injection mass flow rate is determined using the CTF-predicted liquid density, facility flow area, and liquid velocity specified in the experiment. CTF is run, the outlet flow distribution is obtained, and this is used as the inlet distribution for the next simulation. This process is repeated until cross-flow ceases throughout the facility.

The friction correlation will drive the flow distribution, as shown later in Section 5.2.2.1. The author indicates that the Sadatomi friction factor correlation [17] leads to the best agreement with the measured flow distribution; however, its complexity makes it difficult to enter into CTF without significant code changes. The author also shows results of the Blasius equation, which 


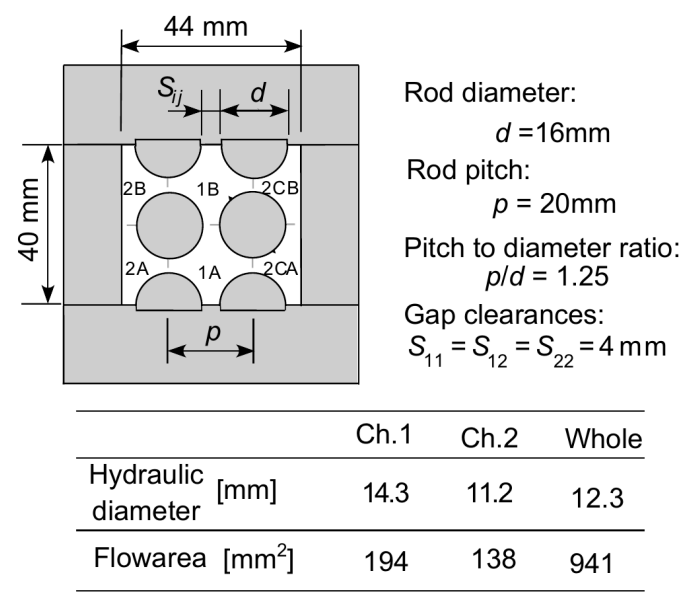

Figure 3.28: Cross-sectional diagram of the $2 \times 3$ facility and relevant geometric information (reprinted from M. Sadatomi et al. "Single- and Two-Phase Turbulent Mixing Rate between Adjacent Subchanels in a Vertical $2 \times 3$ Rod Array Channel". In: International Journal of Multiphase Flow 30 (2004), pp. 481-498.

seems to also perform well. Therefore, the Blasius correlation (Equation 3.16) is used as a first step in this study; however, the CTF friction correlation (Equation 3.17), which is a default model in CTF, is also tested.

$$
\begin{gathered}
f=0.316 R e^{-0.25} \\
f=0.204 R e^{-0.2}
\end{gathered}
$$

The single-phase turbulent mixing coefficient is varied during this study to investigate its impact on mixing results. The inlet temperature is set to $24^{\circ} \mathrm{C}$ and the outlet pressure is set to 1.01325 bar. 


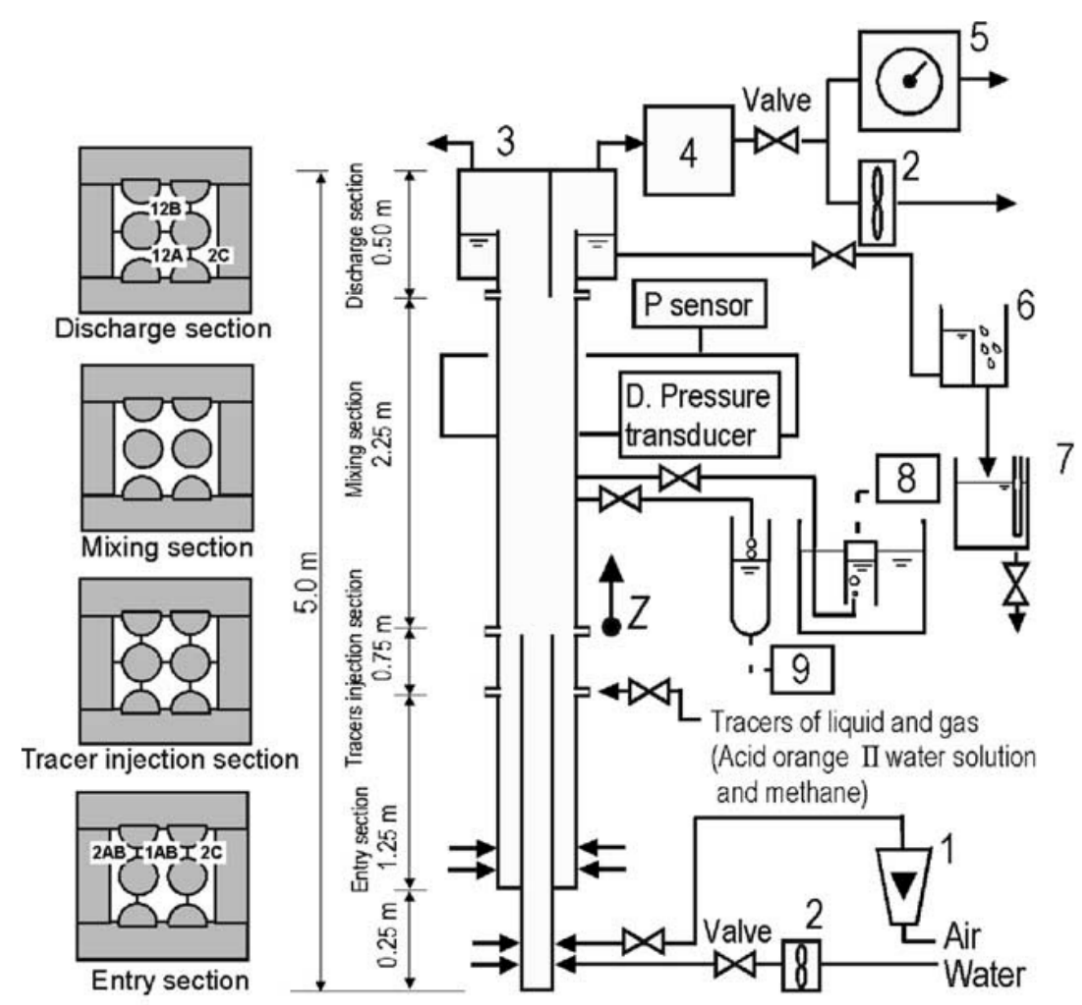

Figure 3.29: Side-view schematic of $2 \times 3$ facility with visualization of channel partitioning in different axial sections (reprinted from M. Sadatomi et al. "Single- and Two-Phase Turbulent Mixing Rate between Adjacent Subchanels in a Vertical $2 \times 3$ Rod Array Channel". In: International Journal of Multiphase Flow 30 (2004), pp. 481-498. 


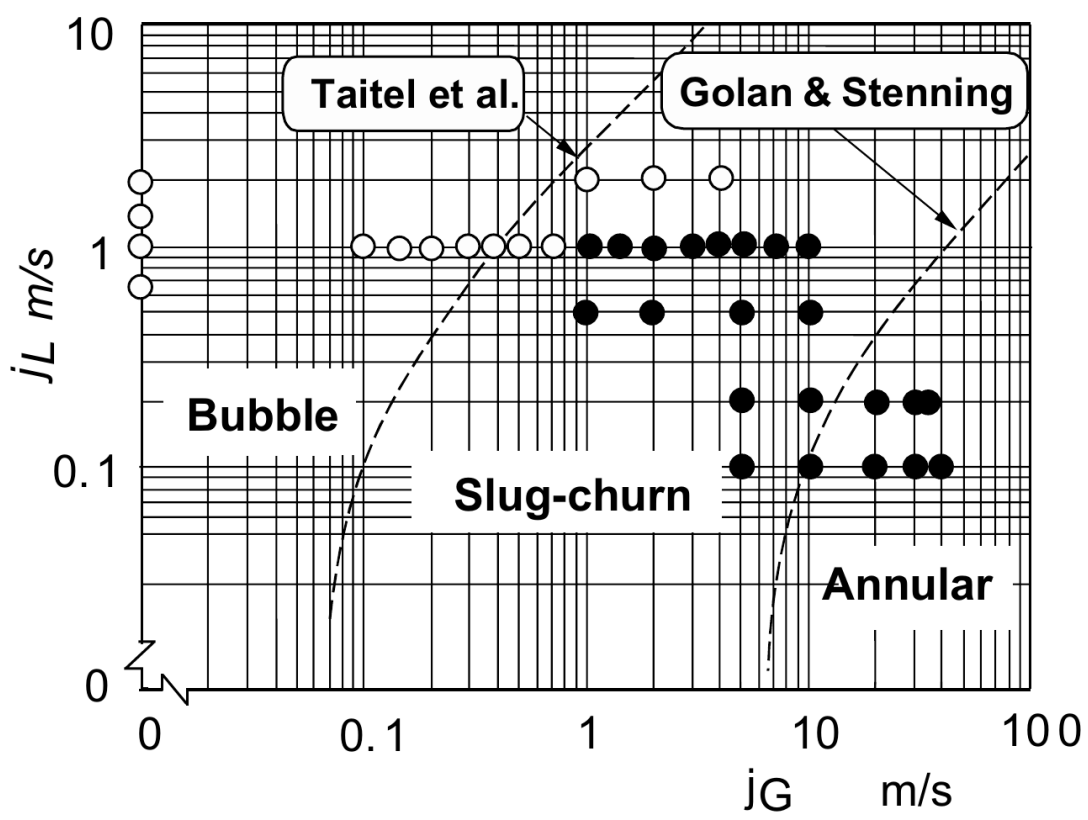

Figure 3.30: Operating conditions for the $2 \times 3$ facility (reprinted from M. Sadatomi et al. "Singleand Two-Phase Turbulent Mixing Rate between Adjacent Subchanels in a Vertical $2 \times 3$ Rod Array Channel". In: International Journal of Multiphase Flow 30 (2004), pp. 481-498. 


\section{CHAPTER 4}

HEAT TRANSFER

When discussing the heat transfer models in the code, what we really care about is the accuracy in the calculation of the Heat Transfer Coefficient (HTC), $h$, that is used in Newton's Law of cooling:

$$
q^{\prime \prime}=h\left(T_{w}-T_{b}\right)
$$

where $q^{\prime \prime}$ is the heat flux from the wall to the fluid, $T_{w}$ is the temperature of the wall surface, and $T_{b}$ is the temperature of the bulk fluid (in the case of a subchannel code, this is the control volume temperature of the fluid).

It is not possible to calculate $h$ directly in experiment but, rather, it must be back-calculated from the experimental measurements of $T_{w}, T_{b}$, and the known rod power of $q^{\prime \prime}$. Since there are difficulties in obtaining values of $T_{b}$ from experiment for the rod-bundle geometry that we are concerned with (associated with holding a thermocouple in a given position of the flow channel and also its impact on the fluid behavior), $T_{b}$ values are typically not available. The $T_{w}$ measurements are much more easily available as the experimenter can insert a thermocouple in the inside of the heater tube, where there is no flow, fix it to the wall, and then post-process the data by solving the 1-D conduction equation for the tube thickness in order to yield the outer tube surface temperature.

In order to actually obtain the experimental value of $h$, this then leaves us with using the subchannel code to estimate values of $T_{b}$ at the $T_{w}$ measurement location so that we can solve Equation 4.1. Of course, this then raises the question of what is the validity of the estimates of $T_{b}$. Rather than introduce this uncertainty into the analysis, we instead directly compare CTF estimates of $T_{w}$ to experimental results. Since the value of the measured wall temperature will be directly related to the magnitude of the convective and boiling heat transfer in the experiment, this is a suitable check on the effectiveness of the employed heat transfer models in CTF. 


\subsection{CE $5 \times 5$}

The CE 5x5 tests involved running over 70 tests of varying operating conditions on a $5 \times 5$ electrically heated rod bundle facility. The operating conditions were varied such that heat transfer mechanisms in the bundle ranged from single-phase convection to saturated boiling. Instrumentation included thermocouples fixed to the interior of two of the heater rods.

In Rod 25, the central heater rod, 14 thermocouples were placed at 4 different levels: 76.63 , $81.00,82.50$, and 83.50 inches from BOHL. One set of thermocouples sat below a mixing vane grid and the other three sets were above the grid. The set below the grid included two thermocouples placed at two different azimuthal locations. The three sets above the grid each included 4 thermocouples placed at 4 azimuthal locations. In Rod 24, the adjacent rod to the west of Rod 25, 4 thermocouples were placed. A single thermocouple was placed below the grid and two sets of thermocouples were placed at two levels above the grid.

Using the temperature for a given thermocouple over all the testing conditions clearly reveals the incipience of boiling at that position in the bundle with a leveling-off of the temperature profile with respect to bundle heat flux. Figure 4.1 shows this axial profile along with the difference between CTF rod surface temperature predictions and the experimental results. This figure also shows another important thing, which is the existence of outliers in the data. Like for the exit channel temperatures, outliers were eliminated from the data set before doing any data analysis. Again, a discrepancy of greater than $50 \mathrm{~F}$ resulted in the data set being removed.

After eliminating outliers, some data reduction was done to generate a more meaningful overview of the predictive capabilities of CTF. First, the merit of interest is the difference between predicted and measured rod surface temperatures. Second, the mean of this discrepancy was calculated for each thermocouple for all tests that were run, leaving us with one mean discrepancy per thermocouple (20 in total). These discrepancies are presented in two figures - one figure for each of the two instrumented rods. Rod 25 results are given in Figure 4.2 and Rod 24 results are given in Figure 4.4 .

In the figures, the x-axis shows the axial location of the thermocouple. The thermocouples, as placed in the figure, are not exactly accurate to their placement in experiment. The red thermocouple (Position 1) is placed at the actual location in the experiment. The following three thermocouples are shifted over to the right slightly to aid in viewing the data. In reality, all thermocouples for a given axial location are at exactly the same axial position in the bundle. The y-axis is the difference between predicted and experimental rod surface temperatures. The two vertical blue lines represent the bottom and the top of the spacer grid. Finally, the error bars do not represent the scatter in the discrepancies for each mean discrepancy. Rather, they represent the $2 \sigma$ scatter of the experimental measurements during the calibration phase of the experiment. For example, in Figure 4.2, the blue thermocouple before the grid has a scatter in measurements of over $5 \mathrm{~F}$. The mean discrepancy between predicted and experimental data is about $-5 \mathrm{~F}$. Considering the scatter in the thermocouple readings, the actual discrepancy may be anywhere between -10 and $1 \mathrm{~F}$.

From the results, we can see that Rod 25 rod temperature predictions are all lower than experimental results, which means that CTF is over-predicting the heat transfer from the rod. As a note, the grid enhancement model was not turned on for these experiments, which should cause an under-prediction in heat transfer downstream of the grid. Actually, we don't see any 


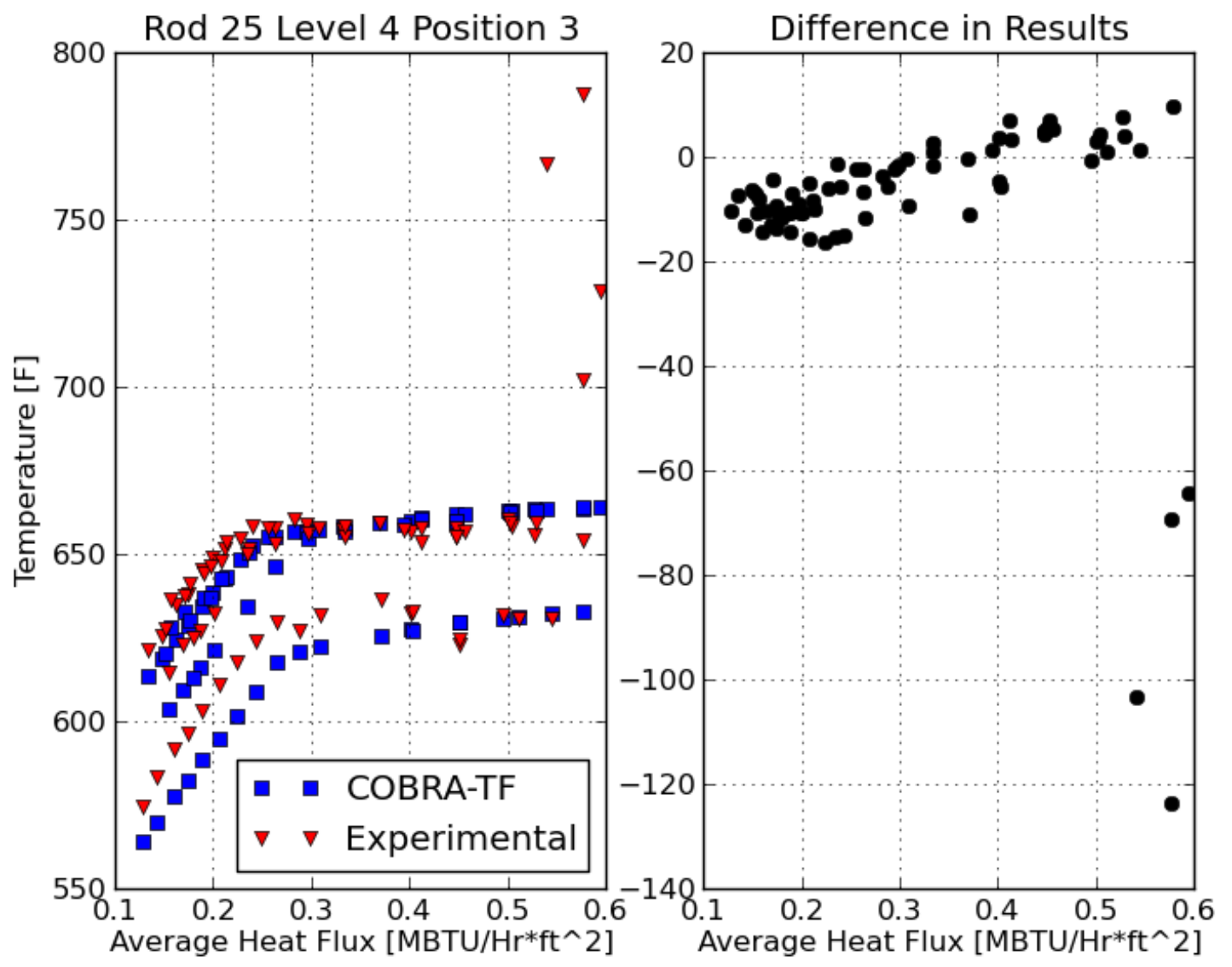

Figure 4.1: Rod surface measurements and predictions with respect to increasing test heat flux for Rod 25 (central rod) at 77.63 inch axial location 


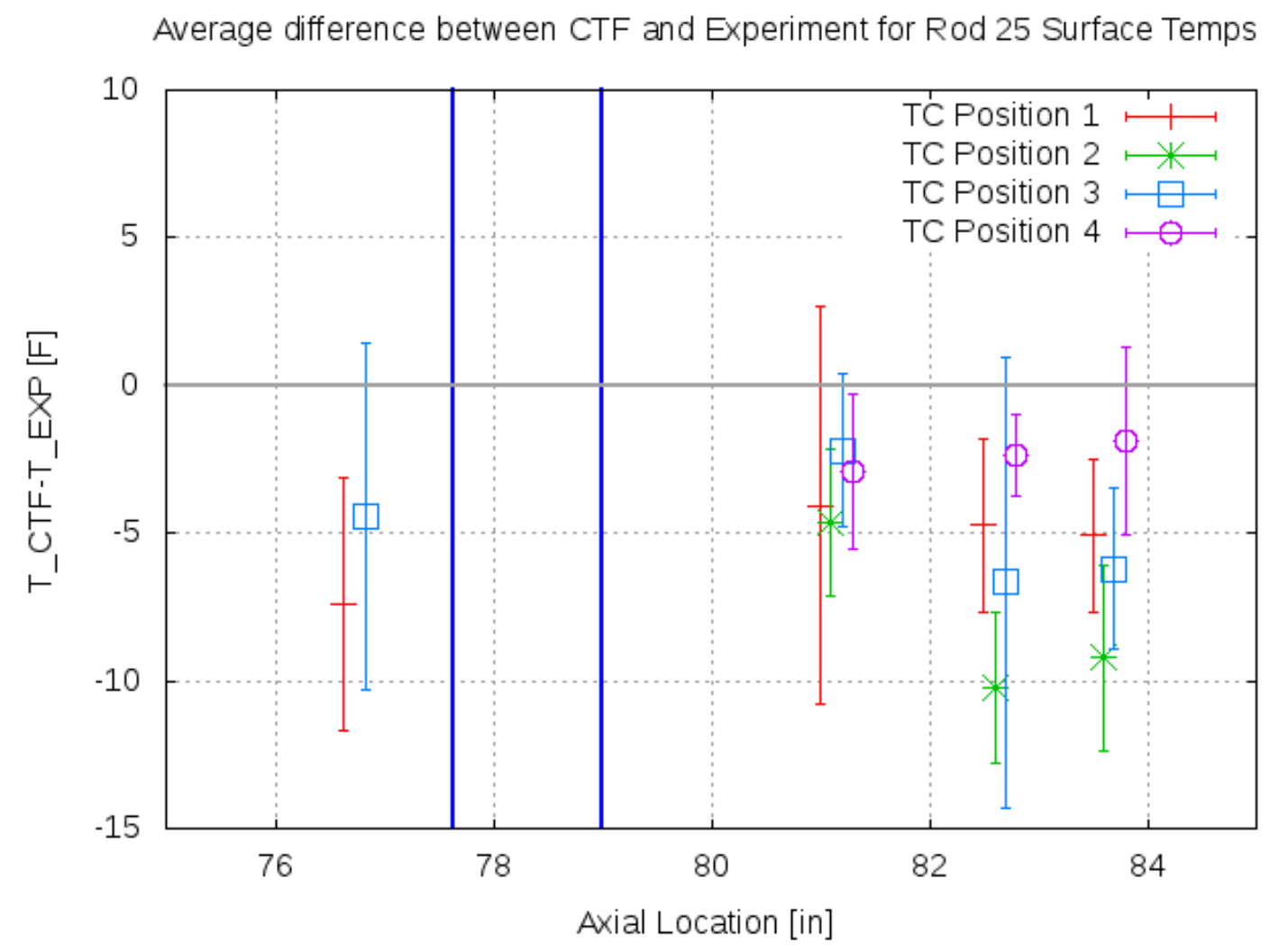

Figure 4.2: Summary of mean difference between predicted and measured rod surface temperatures for each thermocouple in Rod 25 over all test cases 
trend in the data before or after the grid in these results. We do see a significant degree of azimuthal variation in the rod surface temperatures (as high as $12 \mathrm{~F}$ at the 83.5 inch location), which we should expect given that the test section uses Mixing Vane Grid (MVG)s (see Figure 4.3). It is important to note that CTF has no mixing vane grid models to account for these types of effects. However, some of this scatter must also be related to uncertainty in the experimental measurements, as the level prior to the grid sees a $3 \mathrm{~F}$ azimuthal variation. Also, we see that, in many cases, the $2 \sigma$ scatter in experimental measurements is enough to account for this azimuthal variation.

Overall, for Rod 25, the mean difference between CTF predictions and measured wall temperatures is $-5.14 \mathrm{~F}$. For Rod 24, the under-prediction of wall temperature is less severe, averaging out to $-1.71 \mathrm{~F}$. 


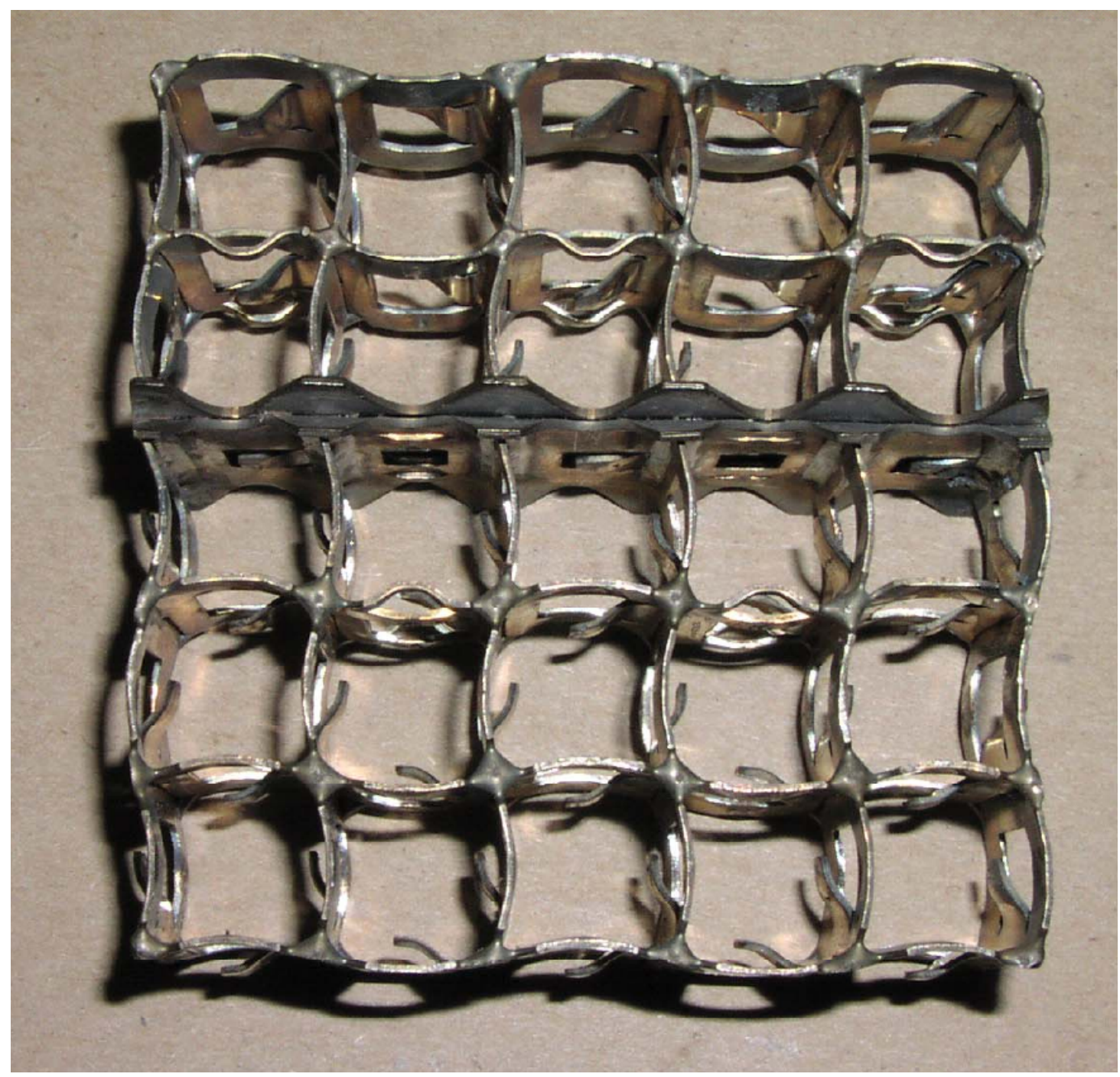

Figure 4.3: CE 5x5 Test 74 Mixing Vane Grid 


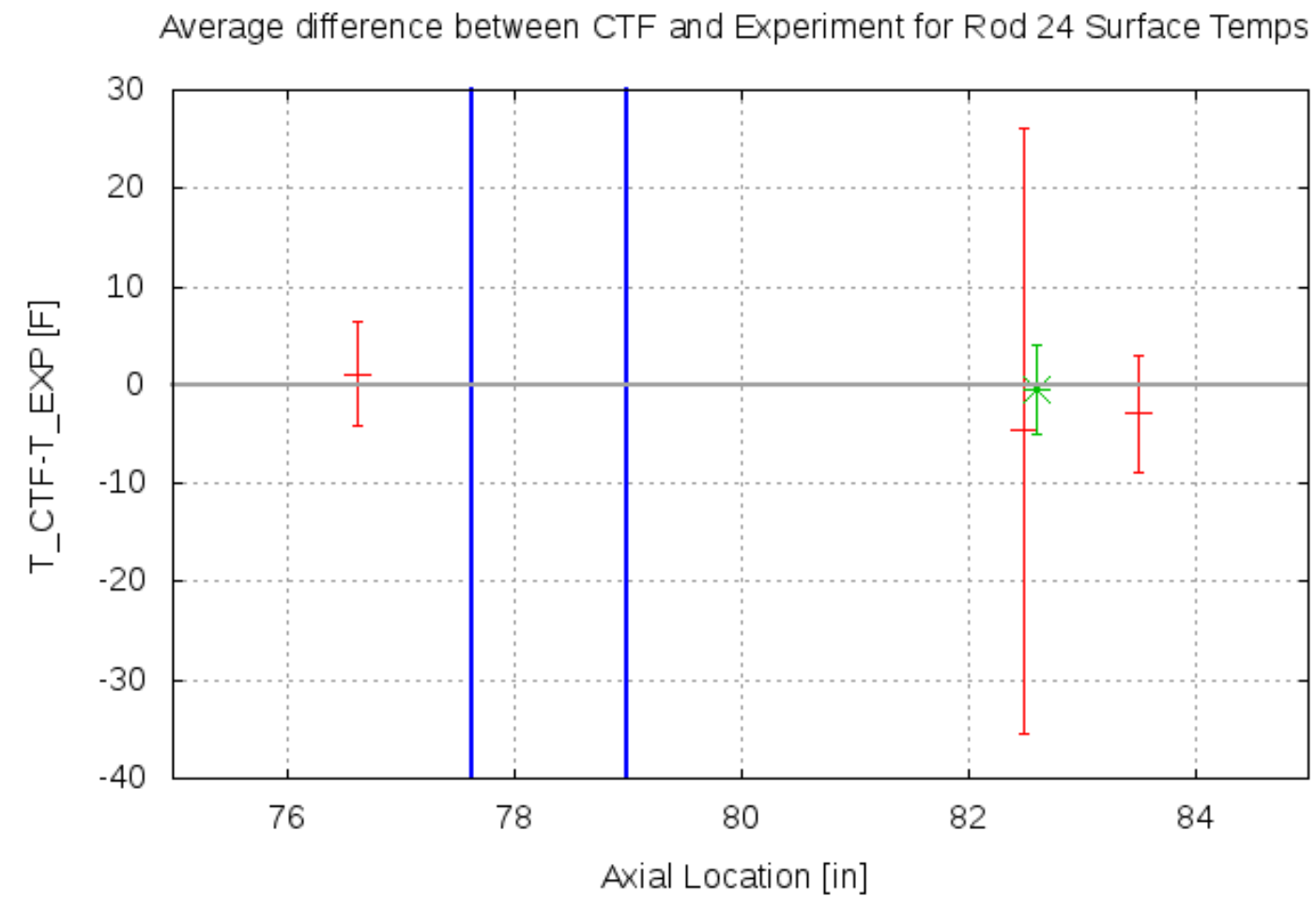

TC Position $1 \longmapsto$ TC Position $2 \longmapsto$

Figure 4.4: Summary of mean difference between predicted and measured rod surface temperatures for each thermocouple in Rod 24 over all test cases 


\section{CHAPTER 5}

PRESSURE LOSS

\subsection{Pressure drop in CTF}

Prior to discussing results, a discussion of CTF-calculated pressure drop is included. Some of the experimental results present only a component of pressure drop (e.g. frictional and acceleration pressure drop, having the gravitational component subtracted off of measured results during experimentation). CTF only solves for a single, total pressure in the model, but will print out the pressure drop components (gravitational, frictional, and acceleration) to an output file. The individual components of pressure drop are calculated upon completion of the simulation. Considering this, it is prudent to discuss the calculation of these terms prior to their use.

The acceleration pressure drop is calculated as the change in the momentum flux across a scalar mesh cell in the code.

$$
\Delta P_{\text {accel }}=\left(\frac{G_{m}^{2}}{\rho_{m}^{+}}\right)_{\text {out }}-\left(\frac{G_{m}^{2}}{\rho_{m}^{+}}\right)_{\text {in }}
$$

The momentum flux term is calculated as:

$$
\frac{G_{m}^{2}}{\rho_{m}^{+}}=\rho_{v} \alpha_{v} v_{v}^{2}+\rho_{l} \alpha_{l} v_{l}^{2}+\rho_{l} \alpha_{d} v_{d}^{2}
$$

Here, $\alpha$ is the phase volume fraction, $v$ is the phase velocity, and $\rho$ is the phase density. The subscripts, $v, l$, and $d$ stand for vapor, liquid, and droplets, respectively. In CTF, the momentum flux is calculated at the boundary of each mesh cell. Therefore, by taking the difference between momentum fluxes at two adjacent axial locations, we obtain the acceleration pressure drop over that corresponding scalar mesh cell. 
The gravitational pressure drop is calculated for the scalar mesh cell as follows:

$$
\Delta P_{\text {gravity }}=\rho_{\text {mix }} g \Delta X
$$

Here, $\Delta X$ is the axial length of the scalar cell, $g$ is the acceleration due to gravity, and $\rho_{\text {mix }}$ is the mixture density, which is calculated as follows:

$$
\rho_{\text {mix }}=\rho_{v} \alpha_{v}+\rho_{l}\left(\alpha_{l}+\alpha_{d}\right)
$$

Finally comes the frictional pressure drop. It is simply calculated by subtracting the gravitational and acceleration losses from the total pressure drop calculated by CTF.

$$
\Delta P_{\text {friction }}=\Delta P_{\text {total }}-\Delta P_{\text {accel }}-\Delta P_{\text {gravity }}
$$

A final note on the calculation of pressure drop terms; these results were averaged across the whole cross-section of the model for a given axial level using an area-weighting approach.

\section{$5.2 \quad$ Single-phase pressure drop}

\subsubsection{Validation}

\subsubsection{BFBT}

The ratio of measured-to-predicted pressure drop for all measurement locations are plotted against test Reynolds number in Figure 5.1. Note that the specification presented these pressure drop results as frictional loss only; the gravitational loss between pressure taps was subtracted off of the total loss before reporting data. Since CTF gives total pressure drop, it was necessary to subtract the gravitational component from the total loss between pressure tap locations.

From these results, we can see that the lower Reynolds test cases are generally under-predicted by CTF. As Reynolds increases past 100,000, the data seems to cluster better around the measurements. To more quantitatively capture the discrepancies between measured and predicted pressure drops, two statistics are shown in Table 5.1: the RMSE and the rRMS for each pressure tap. In addition to giving a discrepancy for each individual pressure tap, a total discrepancy for all test data is given in the last row of the table.

The RMSE metric is actually somewhat misleading because the collection of measurements taken at a given pressure tap were taken over a range of operating conditions. As the mass flow rate in the system increases, the pressure drop over a given tap increases as well, and with increasing pressure drop, we obtain increasing measurement error. Likewise, we expect more discrepancy between measured and predicted values as pressure drop increases. Therefore, it is not entirely fair to compare the RMSE of one pressure tap to that of another, as dp309, for example, which covers most of the test section, will have a much higher total pressure drop than 


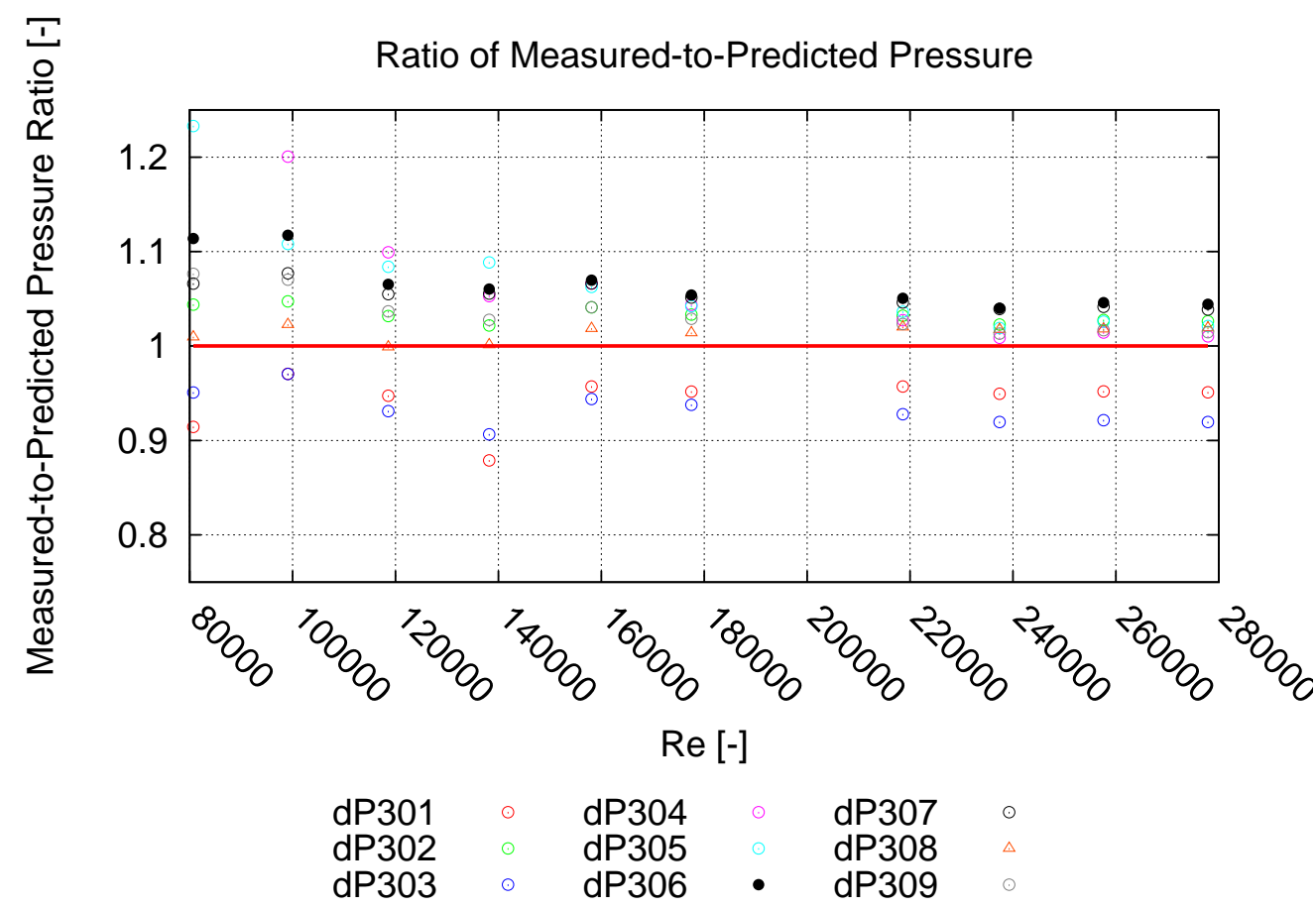

Figure 5.1: BFBT single-phase pressure drop predictions (Series 7)

dp301, which covers only 1 grid span. Inspection of Figure 5.1 clearly shows that the relative discrepancy between measured and predicted results for dp309 are slightly less than the relative discrepancy between measured and predicted results for dp301, actually. However, the RMSE in Table 5.1 would indicate otherwise, showing a discrepancy that is over 3 times higher for dp309.

This rRMS puts things into perspective by generating the average relative error for each pressure tap. We see that the average relative error of dp309 is only $3.9 \%$, which is better than dp301 discrepancy of $6.8 \%$ as we expect. We also see that the pressure taps with the largest sources of error are dp303 and dp306. From Figure 5.1, we see that, in the case of dp303, CTF is over-predicting results and, in the case of dp306, CTF is under-predicting results. It is peculiar that this is the case, because the spans covered by these two pressure taps are about the same size (dp303 span is $466 \mathrm{~mm}$ and dp306 span is $512 \mathrm{~mm}$ ). Additionally, both spans contain one grid. The experimental uncertainty for pressure drop given in the specification was $1 \%$; the total rRMS value of Table 5.1 of $5 \%$ is far outside of this range.

The BFBT tests contained both single-phase and two-phase pressure drop experiments. Single phase pressure drop results obtained from Series 7 are presented first, followed by two-phase pressure drop results from Series 6. 
Table 5.1: RMSE and rRMS for measured and predicted data of BFBT P7 single-phase pressure drops

\begin{tabular}{|l|l|c|}
\hline Pressure Tap & RMSE (kPa) & rRMS (\%) \\
\hline dp301 & 0.097 & 6.8 \\
dp302 & 0.056 & 3.3 \\
dp303 & 0.188 & 7.5 \\
dp304 & 0.078 & 10.0 \\
dp305 & 0.087 & 8.1 \\
dp306 & 0.121 & 6.6 \\
dp307 & 0.326 & 5.2 \\
dp308 & 0.050 & 1.6 \\
dp309 & 0.364 & 3.9 \\
\hline total & 0.188 & 6.4 \\
\hline
\end{tabular}

\subsubsection{Verification}

\subsubsection{Single-Phase Friction Flow Split}

In CTF, there are three mechanisms for lateral flow between channels: pressure differences, turbulent-mixing, and void-drift. The user has the option to shut off the turbulent-mixing and void-drift independently of the pressure-driven cross-flow effects. If the turbulent-mixing and void-drift models are disabled, flow redistribution can only occur due to lateral pressure differences in the model. Lateral pressure gradients can arise due to lateral density differences (due to non-uniform radial heating in the model), due to non-uniform inlet boundary conditions, or due to lateral boundary conditions. If we create an unheated model with uniform inlet boundary conditions, lateral pressure gradients will form due to differences in pressure drops in adjacent channels.

In CTF, pressure drop is a combination of gravitational, acceleration, form, and friction losses. This problem is built such that wall friction becomes the only driver for cross-flow and, thus, determines the flow distribution between channels. An analytical solution is formed to determine this ideal flow split and the CTF results are compared to the analytical solution.

Problem Description A model of two channels connected by a gap is created. One channel has a larger hydraulic diameter than the other. The inlet velocity is uniform in the two channels, the case is unheated, and the coolant is single-phase and highly subcooled. This creates a difference in Reynolds number at the inlet of the two channels, which creates different frictional pressure drops in the two channels, as the friction model is Reynolds dependent.

The different frictional pressure drops create a lateral pressure gradient that drives flow from the higher resistance channel to the lower resistance channel. Moving up the channels, velocity grows larger in the low-resistance channel, which increases frictional pressure drop in that channel. Simultaneously, velocity decreases in the high-resistance channel, which decreases frictional pressure drop. This continues until the frictional pressure drop is the same in both channels, at which point cross-flow ceases. At this point, the channels are said to be in mechanical 
equilibrium.

An analytical solution is derived for this point of mechanical equilibrium. We consider a control volume in each channel at this level where equilibrium has been reached. It is safe to neglect the lateral momentum equation because cross-flow has stopped. An axial momentum equation can be formed for each channel control volume. The general axial momentum equation is shown below.

$$
\rho\left[\frac{\partial \boldsymbol{V}}{\partial t}+u \frac{\partial \boldsymbol{V}}{\partial x}+v \frac{\partial \boldsymbol{V}}{\partial y}+w \frac{\partial \boldsymbol{V}}{\partial z}\right]=\rho g-\nabla p+\nabla \dot{\tau}_{i j}
$$

The density is removed from the left-hand side terms since it is assumed constant in the problem. The bracketed terms include: 1) time-change of momentum, 2) axial (x) advection of momentum, 3) lateral $(y)$ advection of momentum, and 4) lateral $(z)$ advection of momentum. The three right-hand side terms are the relevant force terms, including: 1) gravity, 2) pressure, and 3) shear.

This equation can be significantly reduced considering

1. the case is steady-state, eliminating the temporal term;

2. there is no cross-flow, eliminating lateral convection terms; and

3. the axial velocity distribution in this control volume is constant, as density is constant and there is no cross-flow, meaning the axial momentum convection term can be eliminated.

This eliminates the entire left-hand side of the equation and leaves the following equation for an individual subchannel, where $x$ is taken as the axial direction:

$$
\frac{d P}{d x}=\rho g+\frac{d \tau_{w}}{d x}
$$

Because the two channels are in mechanical equilibrium, the pressure drops in the channels are equal, allowing us to equate the right-hand side of each individual channel equation. Note that the gravity head is identical in the two channels, allowing the term to be cancelled. Finally, integrating over the control volume height, $d x$, allows us to obtain the final relation between the two channels.

$$
\tau_{w, 1}=\tau_{w, 2}
$$

The wall drag, $\tau_{w}$, is determined from the CTF friction model, which is substituted into Equation 5.8 to produce the following expansion:

$$
\frac{f_{1} u_{1}^{2}}{2 D_{h, 1}}=\frac{f_{2} u_{2}^{2}}{2 D_{h, 2}}
$$


The terms, $f, u$, and $D_{h}$, represent the Darcy friction factor, liquid velocity, and channel hydraulic diameter, respectively. The subscripts indicate which channel the term represents. The CTF friction factor model is used in the problem to calculate $f$ as a function of Reynolds number. It has the following form:

$$
f=C_{1} R e^{C_{2}}
$$

$C_{1}$ and $C_{2}$ are model coefficients. Expanding the Reynolds number and substituting this into Equation 5.9 yields the following relationship between channel velocities:

$$
\frac{C_{1}\left(\frac{\rho u_{1} D_{h, 1}}{\mu}\right)^{C_{2}} u_{1}^{2}}{D_{h, 1}}=\frac{C_{1}\left(\frac{\rho u_{2} D_{h, 2}}{\mu}\right)^{C_{2}} u_{2}^{2}}{D_{h, 2}}
$$

Canceling terms and reducing leads to the following form:

$$
\left(\frac{u_{1}}{u_{2}}\right)^{2+C_{2}}=\left(\frac{D_{h, 2}}{D_{h, 1}}\right)^{C_{2}-1}
$$

The hydraulic diameters of the two channels are defined by the model geometry. For CTF, $C_{2}$ is -0.2. If we also consider the mass conservation equation, which tells us that the sum of the outlet mass flow rates is equal to the inlet mass flow rate, we can obtain the expected solutions for the absolute outlet mass flow rate of each channel. First, Equation 5.12 is set in terms of mass flow rate to produce the following:

$$
\left(\frac{\dot{m}_{1}}{\dot{m}_{2}} \frac{A_{2}}{A_{1}}\right)^{2+C_{2}}=\left(\frac{D_{h, 2}}{D_{h, 1}}\right)^{C_{2}-1}
$$

Next, the mass conservation equation is used to relate the mass flow rates in the individual channels to the total mass flow rate in the system:

$$
\begin{aligned}
& \dot{m}_{\mathrm{in}}=\dot{m}_{1}+\dot{m}_{2} \\
& \dot{m}_{\mathrm{in}}=\dot{m}_{2}\left(1+\left(\frac{D_{h, 2}}{D_{h, 1}}\right)^{\frac{C_{2}-1}{2+C_{2}}} \frac{A_{1}}{A_{2}}\right)
\end{aligned}
$$

CTF Model Description Channel 2 has a hydraulic diameter that is twice the size of the Channel 1 hydraulic diameter. The area and wetted perimeter of Channel 1 are set to values close to those expected of typical PWR rod-lattice geometry. The outlet pressure is 155 bar and inlet mass flux is $3500 \mathrm{~kg} \mathrm{~m}^{-2} \mathrm{~s}^{-1}$. The inlet temperature is set to $200^{\circ} \mathrm{C}$ to keep the model sufficiently subcooled, and the case is unheated. Turbulent mixing and void drift is disabled so that pressure is the only driver for cross-flow. A diagram of this model is shown in Figure 5.2. 


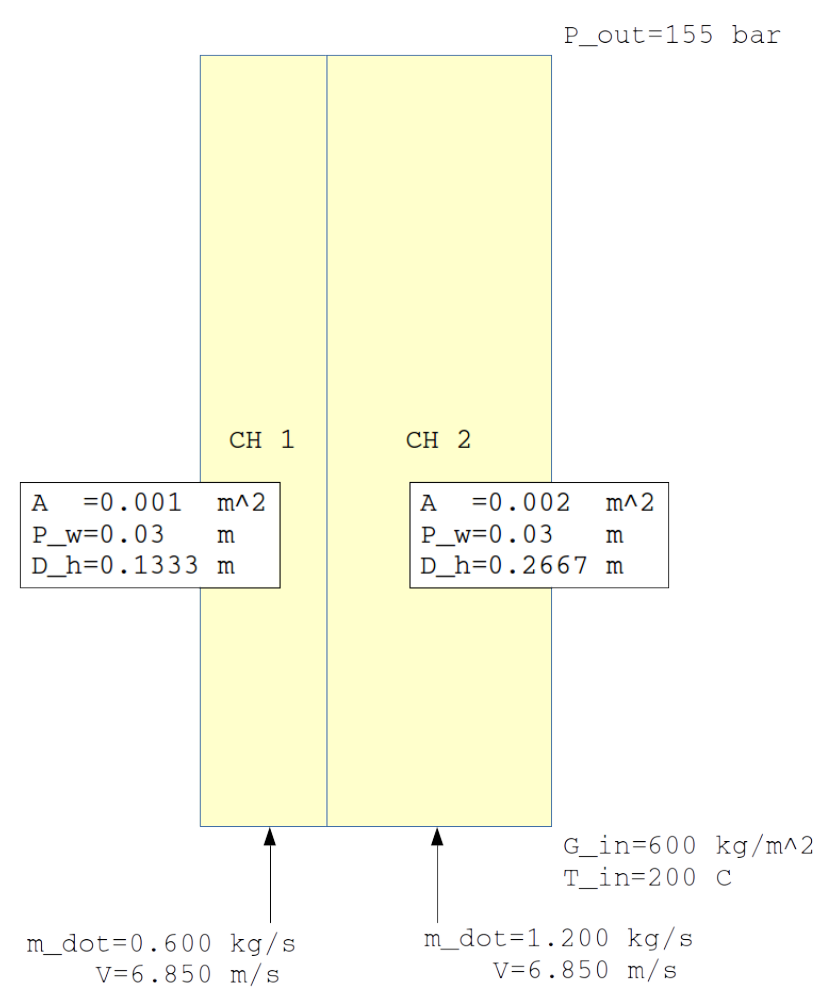

Figure 5.2: Diagram of the two-channel flow split problem.

The length of the model is set to $10 \mathrm{~m}$ to allow the flow to completely redistribute within the CTF solution space.

The axial mesh is set to different sizes, including $2.54 \mathrm{~cm}, 5.08 \mathrm{~cm}$, and $10.16 \mathrm{~cm}$; however, it is found that axial mesh size has no impact on the axial mass flow rate profiles. With the geometry defined, Equation 5.15 is used to calculate that the expected outlet mass flow rates in Channels 1 and 2 .

Discussion of Results The CTF solution includes the axial mass flow rate distribution in each channel. The analytical solution only gives us the expected flow rate distribution at the exit. Therefore, we cannot compare the CTF axial flow distribution to the analytical solution, but we can at least guarantee that CTF achieves the correct flow split when mechanical equilibrium is achieved. The mass flux in each channel is normalized before plotting using the following relationship:

$$
G_{i, \mathrm{norm}}=\frac{G_{i}-\bar{G}}{\bar{G}} \times 100
$$




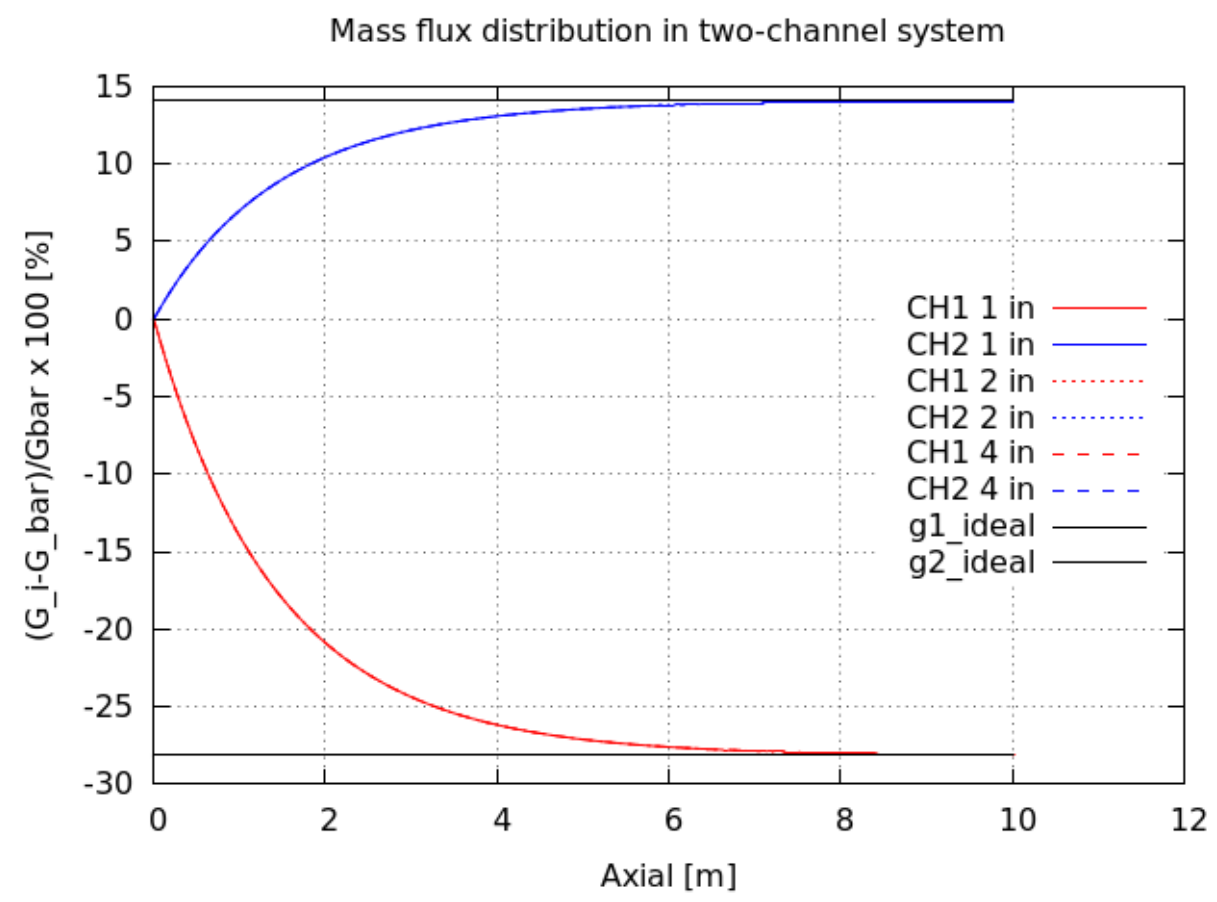

Figure 5.3: CTF-predicted axial mass flux distribution in two-channel system compared with analytical solution.

$\bar{G}$ is the average mass flux between channels, which is equal to the inlet mass flux. Therefore, the normalized mass flux in each channel is zero at the inlet and then re-distributes due to frictional resistance. Figure 5.3 shows the CTF-predicted flow distribution in the two-channel system. The correct analytical flow split is shown with the dashed lines.

These results demonstrate that CTF predicts the expected flow split between the two channels at about $7 \mathrm{~m}$ from the inlet.

\subsubsection{Friction Model Verification}

A suite of tests is defined in this section for verifying that the various friction factor models are correctly implemented in CTF. CTF has four pre-defined friction factor models; two assume all surfaces are smooth and the other two consider the effect of surface roughness. The code also includes a fifth option for the user to specify a custom friction correlation. This set of tests considers various permutations of a $3 \times 3$ rod bundle. Permutations include varying the size of the center guide tube, changing the roughness of the rods, and adding a wall around the bundle.

In considering the effects of surface roughness, CTF considers an area-weighted average of the surface roughness in each channel. Therefore, it should be expected that the meshing of the problem will lead to different results (i.e., a rod-centered subchannel model will experience slightly different frictional losses than a channel-centered subchannel model). Therefore, the $3 \times 3$ 

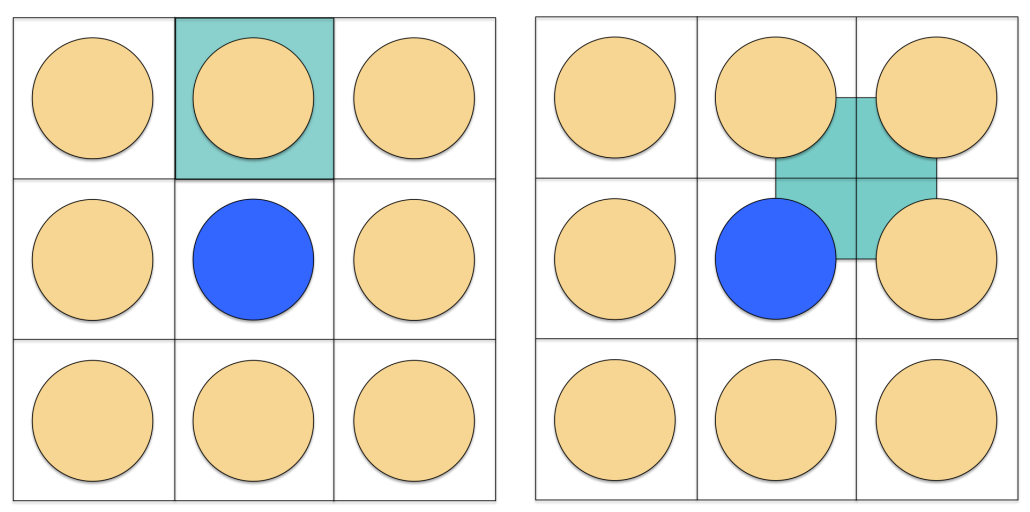

Figure 5.4: $3 \times 3$ rod bundle geometry with (a) rod-centered and (b) channel-centered subchannels.

bundle is modeled in two ways: 1) using a rod-centered approach, and 2) using a channel-centered approach. Each of these sub-series of tests activates each of the four pre-defined friction models in CTF and uses multiple permutations of bundle geometry. The custom friction model is tested by specifying the Blasius correlation as input.

Problem Description A suite of channel-centered and rod-centered models is defined to adequately test the friction models of CTF for several geometries of interest. Figure 5.4 shows the difference between the rod-centered and channel-centered modeling approaches for the $3 \times 3$ bundle. The blue shaded region shows the definition of a channel in the CTF model; as a result, the rod-centered model has 9 channels and the channel-centered model has 16 channels. The blue rod is the guide tube.

The channel-centered model characteristics are shown in Table 5.2. The rod-centered model characteristics are shown in Table 5.3. In each test name, the letter suffix (ranging from "a"-"e") represents the friction model being employed in the study. The number following "test" represents the test series; the geometry is varied slightly between test series. The main perturbations are to vary the guide tube size, the rod roughness, and the presence of a wall around the bundle.

Varying the guide tube size will cause flow area of the channels touching the guide tube to change, resulting in a flow redistribution. Likewise, increasing guide tube roughness causes greater flow resistance in the center of the bundle and causes flow to migrate to the boundary channels. The channel-centered models investigate the effect of including a wall around the bundle. Due to the implementation of the surface-roughness models in CTF, the wall is assumed smooth unless the wall is explicitly modeled as a heated or unheated conductor in the model. Including the wall in the verification tests is necessary to ensure that this intended behavior is correct.

The proper implementation of the friction models is determined by calculating the expected flow split and then comparing this to the CTF simulation results. It is difficult to directly calculate the expected pressure drop in each channel of the model because wall shear is a function of velocity, which changes along the axial length as the flow redistributes. The approach for determining the flow split is similar to Section 5.2.2.1. In these problems, the friction factor 
Table 5.2: Summary of characteristics of channel-centered friction verification tests

\begin{tabular}{lrrrrrr}
\hline Test & $\mathrm{d}_{\text {rod }}[\mathrm{mm}]$ & $\mathrm{d}_{\mathrm{gt}}[\mathrm{mm}]$ & $\epsilon_{\text {rod }}[\mu \mathrm{m}]$ & $\epsilon_{\mathrm{gt}}[\mu \mathrm{m}]$ & Wall & IRFC \\
\hline cc_test1a & 9.5 & 9.5 & 0.0 & 0.0 & No & 1 \\
cc_test1b & 9.5 & 9.5 & 0.0 & 0.0 & No & 2 \\
cc_test1c & 9.5 & 9.5 & 0.0 & 0.0 & No & 3 \\
cc_test1d & 9.5 & 9.5 & 0.0 & 0.0 & No & 4 \\
cc_test1e & 9.5 & 9.5 & 0.0 & 0.0 & No & 5 \\
\hline cc_test2a & 9.5 & 11.22 & 0.0 & 0.0 & No & 1 \\
cc_test2b & 9.5 & 11.22 & 0.0 & 0.0 & No & 2 \\
cc_test2c & 9.5 & 11.22 & 0.0 & 0.0 & No & 3 \\
cc_test2d & 9.5 & 11.22 & 0.0 & 0.0 & No & 4 \\
cc_test2e & 9.5 & 11.22 & 0.0 & 0.0 & No & 5 \\
\hline cc_test3a & 9.5 & 11.22 & 0.0 & 10.0 & No & 1 \\
cc_test3b & 9.5 & 11.22 & 0.0 & 10.0 & No & 2 \\
cc_test3c & 9.5 & 11.22 & 0.0 & 10.0 & No & 3 \\
cc_test3d & 9.5 & 11.22 & 0.0 & 10.0 & No & 4 \\
cc_test3e & 9.5 & 11.22 & 0.0 & 10.0 & No & 5 \\
\hline cc_test4a & 9.5 & 9.5 & 0.0 & 0.0 & Yes & 1 \\
cc_test4b & 9.5 & 9.5 & 0.0 & 0.0 & Yes & 2 \\
cc_test4c & 9.5 & 9.5 & 0.0 & 0.0 & Yes & 3 \\
cc_test4d & 9.5 & 9.5 & 0.0 & 0.0 & Yes & 4 \\
cc_test4e & 9.5 & 9.5 & 0.0 & 0.0 & Yes & 5 \\
\hline cc_test5a & 9.5 & 9.5 & 10.0 & 10.0 & Yes & 1 \\
cc_test5b & 9.5 & 9.5 & 10.0 & 10.0 & Yes & 2 \\
cc_test5c & 9.5 & 9.5 & 10.0 & 10.0 & Yes & 3 \\
cc_test5d & 9.5 & 9.5 & 10.0 & 10.0 & Yes & 4 \\
cc_test5e & 9.5 & 9.5 & 10.0 & 10.0 & Yes & 5 \\
\hline & & & & & \\
\hline
\end{tabular}

Table 5.3: Summary of characteristics of rod-centered friction verification tests

\begin{tabular}{crrrrrr}
\hline \multicolumn{1}{c}{ Test } & $\mathrm{d}_{\text {rod }}[\mathrm{mm}]$ & $\mathrm{d}_{\mathrm{gt}}[\mathrm{mm}]$ & $\epsilon_{\text {rod }}[\mu \mathrm{m}]$ & $\epsilon_{\mathrm{gt}}[\mu \mathrm{m}]$ & Wall & IRFC \\
\hline rc_test1a & 9.5 & 9.5 & 0.0 & 0.0 & No & 1 \\
rc_test1b & 9.5 & 9.5 & 0.0 & 0.0 & No & 2 \\
rc_test1c & 9.5 & 9.5 & 0.0 & 0.0 & No & 3 \\
rc_test1d & 9.5 & 9.5 & 0.0 & 0.0 & No & 4 \\
\hline rc_test2a & 9.5 & 11.22 & 0.0 & 0.0 & No & 1 \\
rc_test2b & 9.5 & 11.22 & 0.0 & 0.0 & No & 2 \\
rc_test2c & 9.5 & 11.22 & 0.0 & 0.0 & No & 3 \\
rc_test2d & 9.5 & 11.22 & 0.0 & 0.0 & No & 4 \\
\hline rc_test3a & 9.5 & 9.5 & 0.0 & 10.0 & No & 1 \\
rc_test3b & 9.5 & 9.5 & 0.0 & 10.0 & No & 2 \\
rc_test3c & 9.5 & 9.5 & 0.0 & 10.0 & No & 3 \\
rc_test3d & 9.5 & 9.5 & 0.0 & 10.0 & No & 4 \\
\hline
\end{tabular}


correlation for some of the models is much more complicated than the form given in Equation 5.10 , so it is not possible to directly solve for the expected velocity distribution in each channel type. Rather, an iterative solution is required to calculate the friction factor using a guessed velocity, then use the friction factor to obtain a more accurate guess of the flow distribution, and so on.

The approach is slightly different depending on whether the model is channel-centered or rod-centered. A rod-centered model has two unique channel types that must be considered: 1) the center channel and, 2) the boundary channels. The system of equations that must be solved for the rod-centered model are:

$$
\begin{aligned}
u_{b, k+1} & =\sqrt{\frac{f_{c}}{f_{b}} u_{c, k}^{2} \frac{D h_{b}}{D h_{c}}} \\
u_{c, k+1} & =\frac{\dot{m}-8 \rho u_{b, k+1} A_{b}}{\rho A_{c}}
\end{aligned}
$$

The subscripts $b$ and $c$ represent "boundary channel" and "center channel", respectively. The subscripts $k$ and $k+1$ represent the current and next iterations in the iterative solution scheme. The friction factor is denoted with $f$, the channel velocity with $u$, the channel flow areas with $A$, liquid density with $\rho$, the total bundle mass flow rate with $\dot{m}$, and the hydraulic diameters with $D h$. The fluid properties are taken directly from the CTF fluid property tables for consistency. The flow areas and hydraulic diameters are calculated from the bundle geometry, which is representative of PWR geometry. The new iteration boundary channel velocity, $u_{b, k+1}$, is relaxed using logarithmic interpolation as shown in Equation 5.19. This is done to smoothly converge on the correct flow distribution and prevent numerical oscillations. The relaxed $u_{b}$ term is used in the mass conservation equation to calculated $u_{c, k+1}$.

$$
u_{b, k+1}=u_{b, k+1}^{0.1} u_{b, k}^{0.9}
$$

The friction factor is calculated from one of the four pre-defined CTF friction factor correlations. They are shown in Equations 5.20-5.23. Note that all correlations return the Darcy friction factor except for the Churchill correlation (Equation 5.23). The Churchill correlation returns the Fanning friction factor, which is four times smaller than the Darcy friction factor. The friction factor correlations are dependent on fluid velocity, which is why an iterative approach is required. The Blasius correlation is used to test the custom input option. It is shown in Equation 5.24.

$$
\begin{gathered}
f=\max \left(1.691 R e^{-0.43}, 0.117 R e^{-0.14}\right) \\
f=0.204 R e^{-0.2} \\
f=\frac{1}{\sqrt{f}}=-2.0 \log _{10}\left(\frac{\epsilon}{3.7 D}+\frac{2.51}{R e}\left[1.14-2 \log _{10}\left(\frac{\epsilon}{D}+\frac{21.25}{R e^{0.9}}\right)\right]\right)
\end{gathered}
$$




$$
\begin{gathered}
f_{w}=2\left[\left(\frac{8}{R e}\right)^{12}+\frac{1}{(a+b)^{3 / 2}}\right]^{1 / 12}, \text { where: } \\
\left.a=\left(2.475 \ln \left[\frac{1}{\left(\frac{7}{R e}\right.}\right)^{0.9}+0.27\left(\frac{\epsilon}{D_{h}}\right)\right]\right)^{16} \\
b=\left(\frac{3.753 x 10^{4}}{R e}\right)^{16} \\
f=0.316 R e^{-0.25}
\end{gathered}
$$

In the channel-centered system, there are three unique channel types: 1) corner channel, 2) side channel, and 3) inner channel. The system of equations for the channel-centered model is as follows:

$$
\begin{aligned}
u_{c, k+1} & =\frac{\dot{m}}{4 \rho A_{c}+8 \sqrt{\frac{f_{c}}{f_{s}} \frac{D h_{s}}{D h_{c}}} \rho A_{s}+4 \sqrt{\frac{f_{c}}{f_{i}} \frac{D h_{i}}{D h_{c}}} \rho A_{i}} \\
u_{s, k+1} & =\sqrt{\frac{f_{c}}{f_{s}} u_{c, k+1}^{2} \frac{D h_{s}}{D h_{c}}} \\
u_{i, k+1} & =\sqrt{\frac{f_{s}}{f_{i}} u_{s, k+1}^{2} \frac{D h_{i}}{D h_{s}}}
\end{aligned}
$$

The subscripts $c, s$, and $i$ represent the "corner", "side", and "inner" type channels, respectively. All other nomenclature is the same as it was for the rod-centered approach. The $u_{c, k+1}$ velocity is also relaxed after each iteration, similar to what was shown in Equation 5.19. The relaxed term is used in the calculation of $u_{s, k+1}$ and $u_{i, k+1}$.

Python scripts are created for the purpose of performing the iterative solution for each of the tests outlined in Tables 5.2 and 5.3. Additionally, the scripts read CTF output files and extract the flow distribution for comparison with the analytical solutions. The scripts can be found in the test directory in the COBRA-TF repository.

CTF Input Model Description The test sections are made sufficiently long so that an equilibrium flow distribution is achieved by the exit of the bundle. Axial meshing is set to $0.2 \mathrm{~m}$, as it was shown in Section 5.2.2.1 that axial mesh size has no impact on the flow redistribution. Outlet pressure is set to 155.13 bar and inlet temperature is set to $292.78^{\circ} \mathrm{C}$. Inlet mass flow rate is set to about $3 \mathrm{~kg} \mathrm{~s}^{-1}$, which corresponds to around $3500 \mathrm{~kg} \mathrm{~m}^{-2} \mathrm{~s}^{-1}$. The test cases are unheated and turbulent mixing and void drift are disabled so that the ideal flow distribution may be achieved.

Discussion of Results Table 5.4 shows the comparison between CTF-predicted exit velocities and the corresponding expected results. Results are shown for each channel type along with the 
Table 5.4: Comparison of CTF-predicted and expected velocity for channel-centered model

\begin{tabular}{|c|c|c|c|c|c|c|c|c|c|}
\hline \multirow[b]{2}{*}{ Test } & \multicolumn{3}{|c|}{ Corner } & \multicolumn{3}{|c|}{ Side } & \multicolumn{3}{|c|}{ Inner } \\
\hline & $\begin{array}{r}\text { Expected } \\
{[\mathrm{m} / \mathrm{s}]}\end{array}$ & $\begin{array}{l}\mathrm{CTF} \\
{[\mathrm{m} / \mathrm{s}]}\end{array}$ & $\begin{array}{r}\text { Error } \\
{[\%]}\end{array}$ & $\begin{array}{r}\text { Expected } \\
{[\mathrm{m} / \mathrm{s}]}\end{array}$ & $\begin{array}{l}\mathrm{CTF} \\
{[\mathrm{m} / \mathrm{s}]}\end{array}$ & $\begin{array}{r}\text { Error } \\
{[\%]}\end{array}$ & $\begin{array}{r}\text { Expected } \\
{[\mathrm{m} / \mathrm{s}]}\end{array}$ & $\begin{array}{l}\mathrm{CTF} \\
{[\mathrm{m} / \mathrm{s}]}\end{array}$ & $\begin{array}{r}\text { Error } \\
{[\%]}\end{array}$ \\
\hline cc_test1a & 4.735 & 4.737 & 0.03 & 5.090 & 5.090 & 0.00 & 5.104 & 5.104 & -0.00 \\
\hline cc_test1b & 4.708 & 4.709 & 0.04 & 5.092 & 5.092 & 0.00 & 5.108 & 5.107 & -0.00 \\
\hline cc_test1c & 4.716 & 4.718 & 0.04 & 5.091 & 5.091 & 0.00 & 5.106 & 5.106 & -0.00 \\
\hline cc_test1d & 4.717 & 4.719 & 0.05 & 5.091 & 5.091 & 0.00 & 5.106 & 5.106 & -0.01 \\
\hline cc_test1e & 4.683 & 4.686 & 0.06 & 5.094 & 5.094 & 0.00 & 5.111 & 5.111 & -0.01 \\
\hline cc_test $2 \mathrm{a}$ & 5.071 & 5.072 & 0.02 & 5.450 & 5.450 & 0.00 & 5.063 & 5.063 & -0.00 \\
\hline cc_test $2 \mathrm{~b}$ & 5.055 & 5.057 & 0.03 & 5.468 & 5.468 & -0.01 & 5.047 & 5.047 & 0.01 \\
\hline cc_test2c & 5.060 & 5.062 & 0.04 & 5.462 & 5.462 & -0.01 & 5.052 & 5.053 & 0.01 \\
\hline cc_test $2 \mathrm{~d}$ & 5.061 & 5.063 & 0.04 & 5.462 & 5.461 & -0.01 & 5.053 & 5.053 & 0.01 \\
\hline cc_test2e & 5.042 & 5.045 & 0.06 & 5.484 & 5.483 & -0.03 & 5.033 & 5.034 & 0.03 \\
\hline cc_test3a & 5.071 & 5.072 & 0.02 & 5.450 & 5.450 & 0.00 & 5.063 & 5.063 & -0.00 \\
\hline cc_test $3 \mathrm{~b}$ & 5.055 & 5.057 & 0.03 & 5.468 & 5.468 & -0.01 & 5.047 & 5.047 & 0.01 \\
\hline cc_test3c & 5.264 & 5.266 & 0.05 & 5.682 & 5.681 & -0.02 & 4.766 & 4.767 & 0.02 \\
\hline cc_test3d & 5.277 & 5.279 & 0.05 & 5.694 & 5.693 & -0.02 & 4.750 & 4.751 & 0.02 \\
\hline cc_test3e & 5.042 & 5.045 & 0.06 & 5.484 & 5.483 & -0.03 & 5.033 & 5.034 & 0.03 \\
\hline cc_test $4 a$ & 3.164 & 3.166 & 0.05 & 4.285 & 4.285 & 0.01 & 6.253 & 6.252 & -0.01 \\
\hline cc_test $4 \mathrm{~b}$ & 3.029 & 3.031 & 0.06 & 4.213 & 4.214 & 0.03 & 6.355 & 6.353 & -0.02 \\
\hline cc_test $4 \mathrm{c}$ & 3.042 & 3.044 & 0.07 & 4.232 & 4.234 & 0.04 & 6.332 & 6.330 & -0.03 \\
\hline cc_test $4 \mathrm{~d}$ & 3.045 & 3.047 & 0.07 & 4.235 & 4.237 & 0.05 & 6.329 & 6.326 & -0.04 \\
\hline cc_test $4 \mathrm{e}$ & 2.913 & 2.917 & 0.11 & 4.148 & 4.152 & 0.09 & 6.444 & 6.440 & -0.07 \\
\hline cc_test5a & 3.164 & 3.166 & 0.05 & 4.285 & 4.285 & 0.01 & 6.253 & 6.252 & -0.01 \\
\hline cc_test5b & 3.029 & 3.031 & 0.06 & 4.213 & 4.214 & 0.03 & 6.355 & 6.353 & -0.02 \\
\hline cc_test5c & 3.303 & 3.304 & 0.04 & 4.399 & 4.399 & 0.01 & 6.109 & 6.108 & -0.01 \\
\hline cc_test5d & 3.300 & 3.302 & 0.04 & 4.396 & 4.397 & 0.01 & 6.112 & 6.111 & -0.01 \\
\hline cc_test5e & 2.913 & 2.917 & 0.11 & 4.148 & 4.152 & 0.09 & 6.444 & 6.440 & -0.07 \\
\hline
\end{tabular}

relative error between CTF-predicted results and expected results. Relative error is always less than $0.10 \%$. Table 5.5 shows a comparison between the linear pressure drop in the top level of the CTF model and the expected linear pressure drop using the expected velocity distribution and relevant friction correlation. At the exit of the CTF model, the flow is in mechanical equilibrium, and so the pressure drop is constant everywhere in the radial direction. Agreement between expected and CTF results is also good; with answers being identical to three decimal places and relative error staying below $0.15 \%$.

Results for the rod-centered models are shown in Table 5.6 for velocity and in Table 5.7 for linear pressure drop. Similar to the channel-centered results, comparison with expected results is good. 
Table 5.5: Comparison of CTF-predicted and expected linear pressure drop for channel-centered model

\begin{tabular}{lrrr}
\hline Test & $\begin{array}{r}\text { Expected } \\
{[\text { bar } / \mathrm{m}]}\end{array}$ & $\begin{array}{r}\text { CTF } \\
{[\text { bar } / \mathrm{m}]}\end{array}$ & $\begin{array}{r}\text { Error } \\
{[\%]}\end{array}$ \\
\hline cc_test1a & 0.147 & 0.147 & -0.09 \\
cc_test1b & 0.117 & 0.117 & -0.13 \\
cc_test1c & 0.104 & 0.104 & -0.11 \\
cc_test1d & 0.102 & 0.102 & -0.07 \\
cc_test1e & 0.094 & 0.094 & -0.10 \\
cc_test2a & 0.167 & 0.167 & -0.12 \\
cc_test2b & 0.133 & 0.133 & -0.08 \\
cc_test2c & 0.118 & 0.118 & -0.07 \\
cc_test2d & 0.116 & 0.116 & -0.10 \\
cc_test2e & 0.107 & 0.107 & -0.09 \\
cc_test3a & 0.167 & 0.167 & -0.12 \\
cc_test3b & 0.133 & 0.133 & -0.08 \\
cc_test3c & 0.127 & 0.127 & -0.06 \\
cc_test3d & 0.125 & 0.125 & -0.06 \\
cc_test3e & 0.107 & 0.107 & -0.09 \\
cc_test4a & 0.215 & 0.215 & -0.11 \\
cc_test4b & 0.173 & 0.173 & -0.10 \\
cc_test4c & 0.154 & 0.154 & -0.06 \\
cc_test4d & 0.151 & 0.151 & -0.05 \\
cc_test4e & 0.141 & 0.141 & -0.03 \\
cc_test5a & 0.215 & 0.215 & -0.11 \\
cc_test5b & 0.173 & 0.173 & -0.10 \\
cc_test5c & 0.220 & 0.220 & -0.09 \\
cc_test5d & 0.218 & 0.218 & -0.11 \\
cc_test5e & 0.141 & 0.141 & -0.03 \\
\hline
\end{tabular}


Table 5.6: Comparison of CTF-predicted and expected linear pressure drop for rod-centered model

\begin{tabular}{lrrrrrrrr}
\hline & \multicolumn{3}{c}{ Boundary } & & \multicolumn{3}{c}{ Center } \\
\cline { 2 - 3 } \cline { 7 - 9 } Test & $\begin{array}{r}\text { Expected } \\
{[\mathrm{m} / \mathrm{s}]}\end{array}$ & $\begin{array}{c}\text { CTF } \\
{[\mathrm{m} / \mathrm{s}]}\end{array}$ & $\begin{array}{r}\text { Error } \\
{[\%]}\end{array}$ & & $\begin{array}{r}\text { Expected } \\
{[\mathrm{m} / \mathrm{s}]}\end{array}$ & $\begin{array}{r}\text { CTF } \\
{[\mathrm{m} / \mathrm{s}]}\end{array}$ & $\begin{array}{r}\text { Error } \\
{[\%]}\end{array}$ \\
\hline rc_test1a & 4.987 & 4.987 & 0.00 & & 4.987 & 4.987 & 0.00 \\
rc_test1b & 4.987 & 4.987 & 0.00 & & 4.987 & 4.987 & 0.00 \\
rc_test1c & 4.987 & 4.987 & 0.00 & & 4.987 & 4.987 & 0.00 \\
rc_test1d & 4.987 & 4.987 & 0.00 & & 4.987 & 4.987 & 0.00 \\
rc_test2a & 5.281 & 5.281 & 0.00 & & 3.800 & 3.801 & 0.01 \\
rc_test2b & 5.290 & 5.290 & 0.01 & & 3.698 & 3.699 & 0.01 \\
rc_test2c & 5.288 & 5.288 & 0.01 & & 3.720 & 3.721 & 0.02 \\
rc_test2d & 5.288 & 5.288 & 0.01 & & 3.724 & 3.724 & 0.02 \\
rc_test3a & 4.987 & 4.987 & 0.00 & & 4.987 & 4.987 & 0.00 \\
rc_test3b & 4.987 & 4.987 & 0.00 & & 4.987 & 4.987 & 0.00 \\
rc_test3c & 5.089 & 5.090 & 0.01 & & 4.165 & 4.166 & 0.02 \\
rc_test3d & 5.092 & 5.093 & 0.01 & & 4.143 & 4.144 & 0.02 \\
\hline
\end{tabular}

Table 5.7: Comparison of CTF-predicted and expected linear pressure drop for rod-centered model

\begin{tabular}{lrrr}
\hline Test & $\begin{array}{r}\text { Expected } \\
{[\text { bar } / \mathrm{m}]}\end{array}$ & $\begin{array}{r}\text { CTF } \\
{[\text { bar } / \mathrm{m}]}\end{array}$ & $\begin{array}{r}\text { Error } \\
{[\%]}\end{array}$ \\
\hline rc_test1a & 0.141 & 0.141 & -0.11 \\
rc_test1b & 0.112 & 0.112 & -0.06 \\
rc_test1c & 0.100 & 0.100 & -0.12 \\
rc_test1d & 0.098 & 0.098 & -0.12 \\
rc_test2a & 0.157 & 0.157 & -0.06 \\
rc_test2b & 0.125 & 0.124 & -0.09 \\
rc_test2c & 0.111 & 0.111 & -0.08 \\
rc_test2d & 0.109 & 0.109 & -0.12 \\
rc_test3a & 0.141 & 0.141 & -0.11 \\
rc_test3b & 0.112 & 0.112 & -0.06 \\
rc_test3c & 0.104 & 0.103 & -0.08 \\
rc_test3d & 0.102 & 0.101 & -0.11 \\
\hline
\end{tabular}


Conclusions An extensive set of tests is applied to CTF in this study. The four pre-defined friction factor models and their implementation are verified. A $3 \times 3$ bundle with guide tube in the center is used as the base model. Several perturbations of this model are made by changing guide tube dimensions, rod roughness, and the presence of a rod bundle wall. All of these changes cause changes to the flow distribution due to frictional pressure drop. Because void drift and turbulent mixing are disabled, the flow distribution is solely defined by the frictional losses. The ideal flow split is analytically calculated for the test suite in this study and results are compared to CTF predictions. CTF-predicted velocity matches expected results within $0.1 \%$ relative error for both rod-centered and channel-centered models. CTF-predicted linear pressure drop matches expected results within $0.15 \%$ for all models. This study indicates proper implementation of the friction factor models in CTF.

\subsection{Two-phase pressure drop}

\subsubsection{Validation}

\subsubsection{BFBT}

The ratio of measured-to-predicted pressure drop is shown in Figure 5.5 with respect to bundleaveraged exit quality. The higher disagreement of Pressure taps 1, 2, and 3 become very evident in this figure and it seems there is very little correlation between the discrepancy and test quality.

Table 5.8 shows the RMSE and rRMS of the pressure drop differences grouped by pressure tap. The last row of the tables presents the statistics for the entire data set taken from all pressure taps and all test cases. From Table 5.8, we see that the first three pressure taps deviate most severely from the measured results. These particular spans are at the top of the bundle where void would be highest. For other spans, the rRMS is much more reasonable at around $5 \%$. The total bundle pressure drops match experimental results (dp309) to within an rRMS of $2.9 \%$, which is very close to the rRMS obtained for dp309 in the single phase tests. Taking all data points into consideration leads to an rRMS of $11.0 \%$. Like for the single-phase cases, experimental uncertainty was quoted to be $1 \%$. The total bundle pressure drop matches experimental results fairly closely; it's the top span locations that lead to the large deviations from experimental results.

The average bundle-exit quality was also measured in the experiments. This result was also compared to CTF predictions. A figure comparing the measured to predicted values for the modeled tests is shown in Figure 5.6. Dashed lines are provided in the figure to act as a guidepost of sorts, showing where $\pm 5 \%$ lies in the figure; the experimental uncertainty of the exit quality was not explicitly stated in the specification. The RMSE for the exit quality for all tests was $0.730 \%$ (note, this represents an average variation of 0.00730 in predicted exit quality from measured exit quality, indicating close agreement in predicted and measured results). 


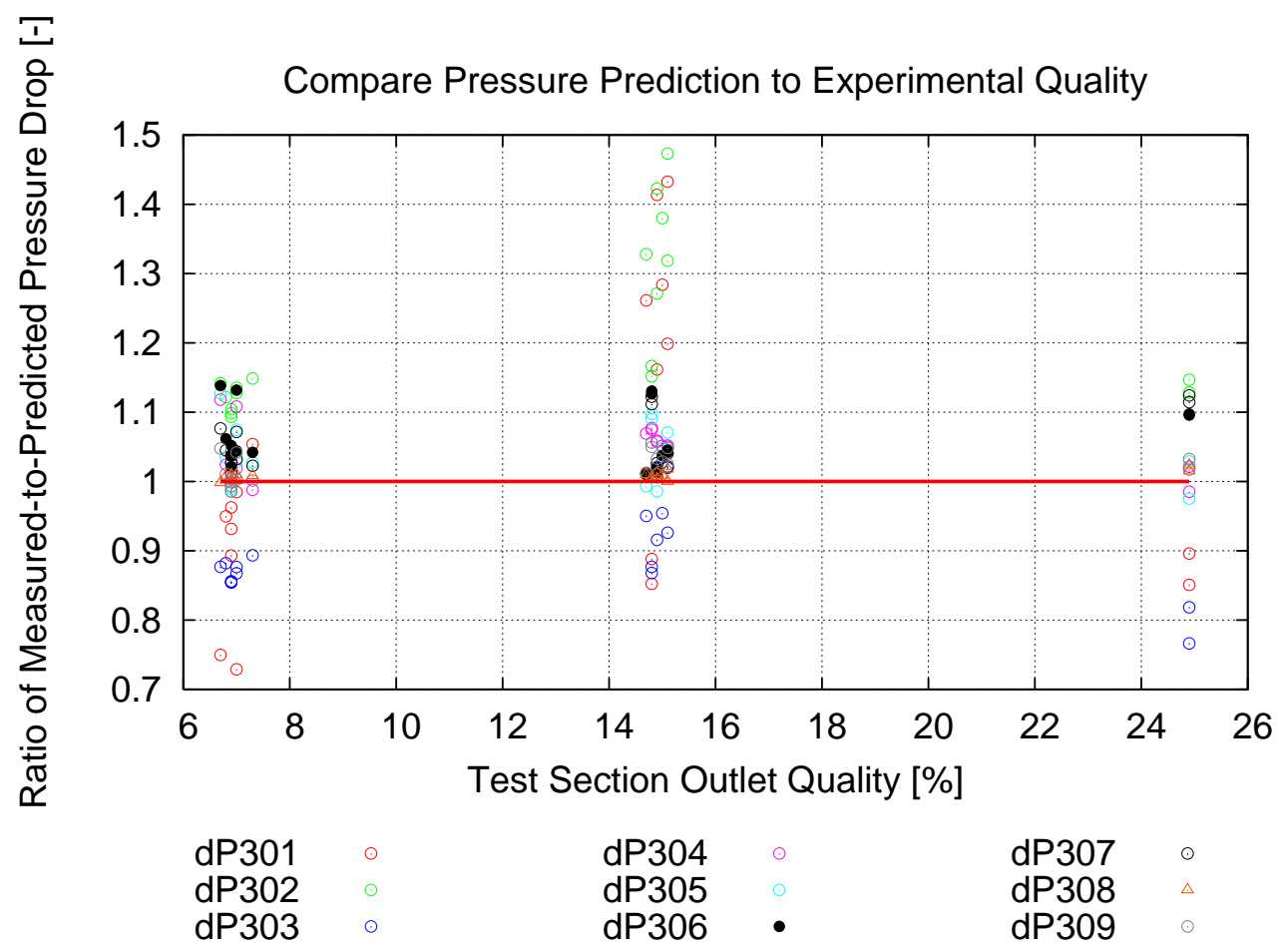

Figure 5.5: Ratio of measured-to-predicted pressure drop compared to bundle-average exit quality for BFBT two-phase tests (Series P6)

Table 5.8: RMSE and rRMS of predicted and measured data of BFBT P6 Series two-phase pressure-drop measurements

\begin{tabular}{|l|c|c|}
\hline Pressure Tap & RMSE (kPa) & rRMS $(\%)$ \\
\hline dp301 & 1.596 & 19.3 \\
dp302 & 8.550 & 18.4 \\
dp303 & 0.920 & 15.0 \\
dp304 & 0.295 & 3.5 \\
dp305 & 0.336 & 4.1 \\
dp306 & 0.365 & 6.4 \\
dp307 & 0.740 & 5.2 \\
dp308 & 0.465 & 6.4 \\
dp309 & 1.676 & 2.9 \\
\hline total & 1.123 & 11.0 \\
\hline
\end{tabular}




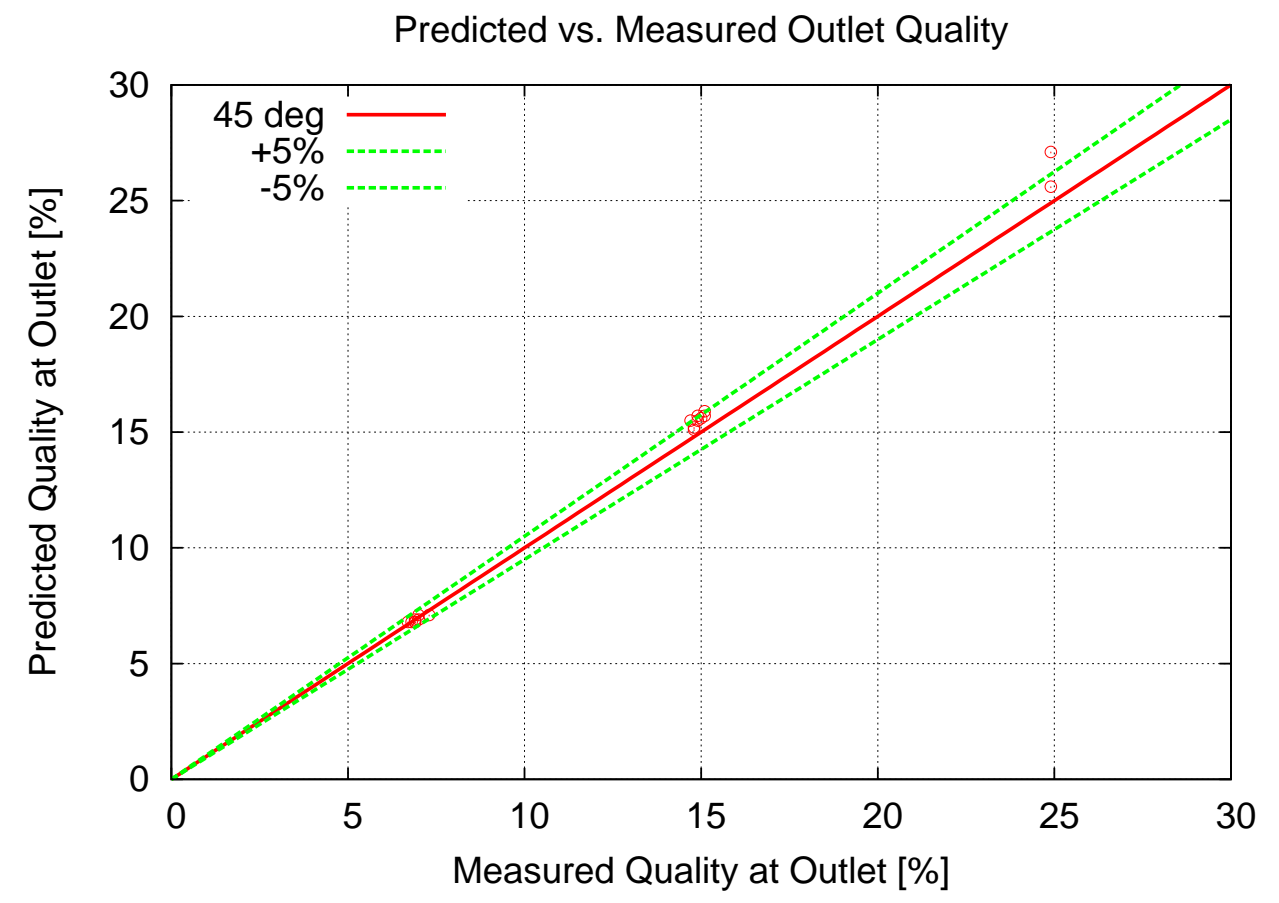

Figure 5.6: Comparison of measured and predicted bundle-averaged exit quality for BFBT P6 Series 


\subsubsection{FRIGG}

Cumulative pressure drop was broken up into three components in the experiment report: acceleration pressure drop, friction pressure drop, and gravitational pressure drop.

The comparison of the three pressure drop terms are shown in Figure 5.7. It's useful to note that pressure is shown in a cumulative fashion. In other words, the acceleration pressure drop profile includes both acceleration and gravitational pressure drop. Further, the friction pressure drop includes the effects of all three components. So, in reality, what is shown as the friction pressure drop is also the total pressure drop of the system. As this figure shows, CTF was able to match both the magnitude and behavior of each of the three components, in addition to the overall pressure drop of the system.

It should be noted, however, that the quantities used to plot experimental pressure drops were obtained from the original report using a digitizer which will contain a certain level of unknown error. Furthermore, the authors of the specification were not clear about how the components of the total pressure drop were calculated. Because of these factors, only the total pressure drop was considered in performing quantitative analysis. Taking the rRMS of the relative errors leads to an average difference of $6.3 \%$ per sampled data point. 


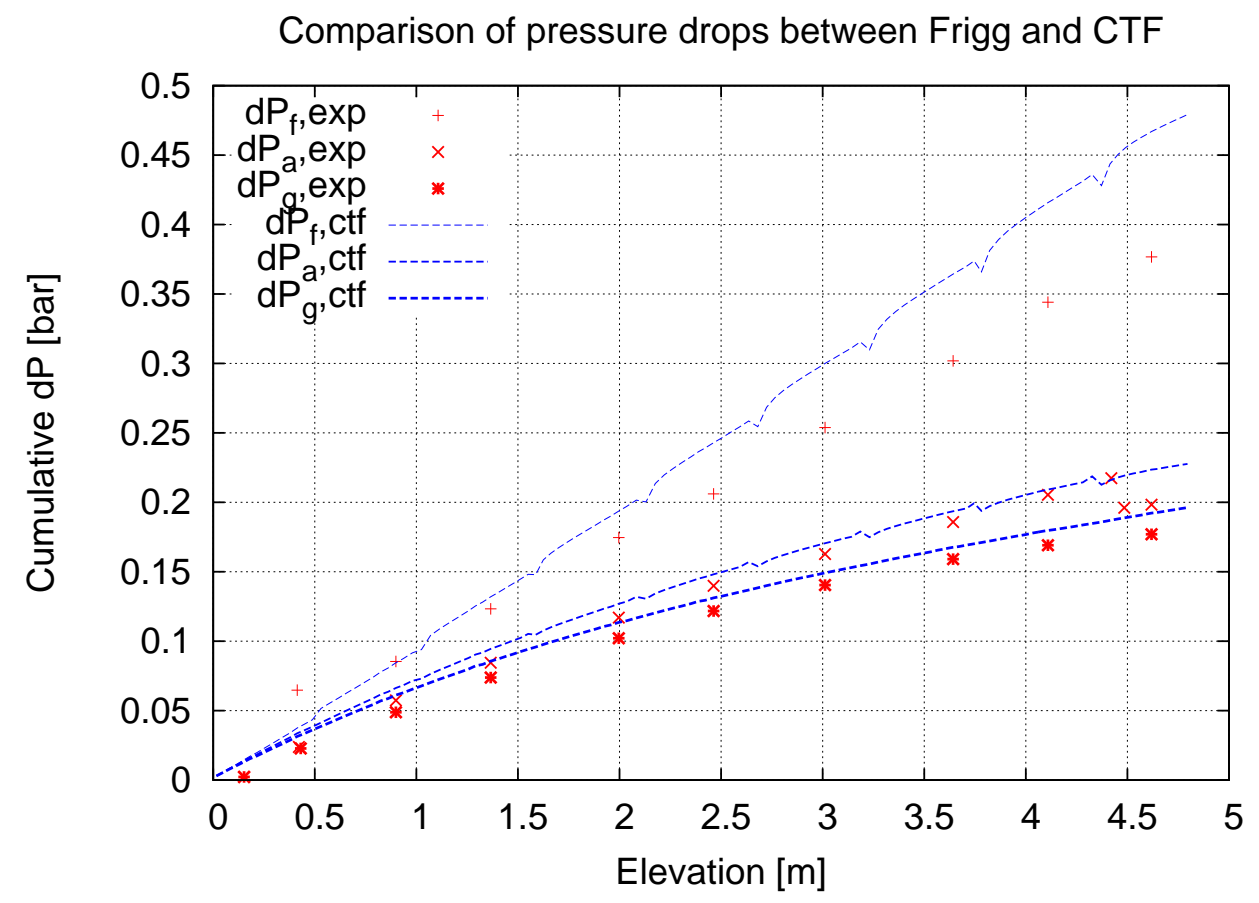

Figure 5.7: Comparison of experimental and predicted components of pressure drop 


\section{CHAPTER 6}

\section{SINGLE-PHASE TURBULENT MIXING}

\subsection{Validation}

\subsubsection{CE $5 \times 5$}

The CE $5 \times 5$ tests were performed for the purpose of assessing heat transfer models. Test conditions were run such that all regions of the boiling curve were experienced from single-phase convection up to Critical Heat Flux (CHF). Temperature measurements were made on the rod surfaces via thermocouples attached to the inside of the heater tubes. However, it is also possible to assess coolant mixing between channels because thermocouples were also placed at the outlet of the test section in the center of each of the 36 coolant channels.

Test 74 was simulated with CTF, which was actually a collection of over 70 separate experiments with different operating conditions that led to different heat transfer mechanisms. The primary operating condition that was modified was the test heat flux. Each individual test was simulated with CTF, then the predicted outlet temperature of each channel was compared to the measured outlet temperature for that channel in the bundle. This was done for all tests in the series and then a mean difference between predicted and measured values was obtained for each channel. A graphical representation of these mean differences are shown in Figure 6.1.

It is important to note that, prior to doing any data comparison on the outlet temperatures, outlier data points were removed. To demonstrate the existence of outliers, see Figure 6.3, which shows the measured and predicted outlet temperatures for Channel 36 of the test section over all test cases. You can see points for three low heat flux cases that are clearly outliers from the rest of the experimental data. To prevent such data from polluting the summary statistics, any data that led to a discrepancy of more than $50 \mathrm{~F}$ between predicted and measured results was eliminated from the analysis.

Returning to Figure 6.1, this plot is useful in demonstrating two things: first, the mean 
discrepancies fall between $\pm 10 \mathrm{~F}$ and, second, the largest errors seem to occur in the corner and side channels of the bundle. A more quantitative view of the data is presented in Figure 6.2. This figure shows a few important things. First, the data is organized by channel type: red dots represent the side-type channels, green dots represent the inner-type channels, and blue dots represent the corner type channels. Additionally, the data are plotted against channel index on the $\mathrm{x}$-axis. The indexing scheme is such that channel numbers start at the north-west corner of the bundle and increase going west-to-east and north-to-south. What we see in this figure is similar to what we saw in Figure 6.1; the corner and side channel discrepancies are generally further from the experimental results than the inner-type channels.

Additionally, this plot also shows the mean discrepancy with the horizontal pink line; it is calculated as an average of all the channel mean discrepancies after removing the outlier discrepancies. We can see that, in general, CTF is over-predicting the experimental results by 2 F. This is what we would expect because the experimental temperature measurements are made in the center of the subchannel, whereas the CTF predictions are the temperature for the entire fluid control volume. The experimental thermocouple measurements will not capture the higher temperatures of the fluid right next to the rod surface, but the CTF predictions will, which acts to raise the average temperature in the control volume.

Finally, this plot also shows the $2 \sigma$ variation in experimental temperature measurements for each thermocouple. Note, the error bars are not showing the standard deviation in the mean discrepancy that is shown. For example, for Channel 7, we see the discrepancy is about 0.5 $\mathrm{F}$, but the error bars show that the experimental measurements varied by about $0.5 \mathrm{~F}$ during successive measurements taken in the calibration phase of the tests. So the actual discrepancy can be anywhere between 1.0 and $0.0 \mathrm{~F}$ for this thermocouple because we can only rely on the temperature obtained from the thermocouple to within $0.5 \mathrm{~F}$. 


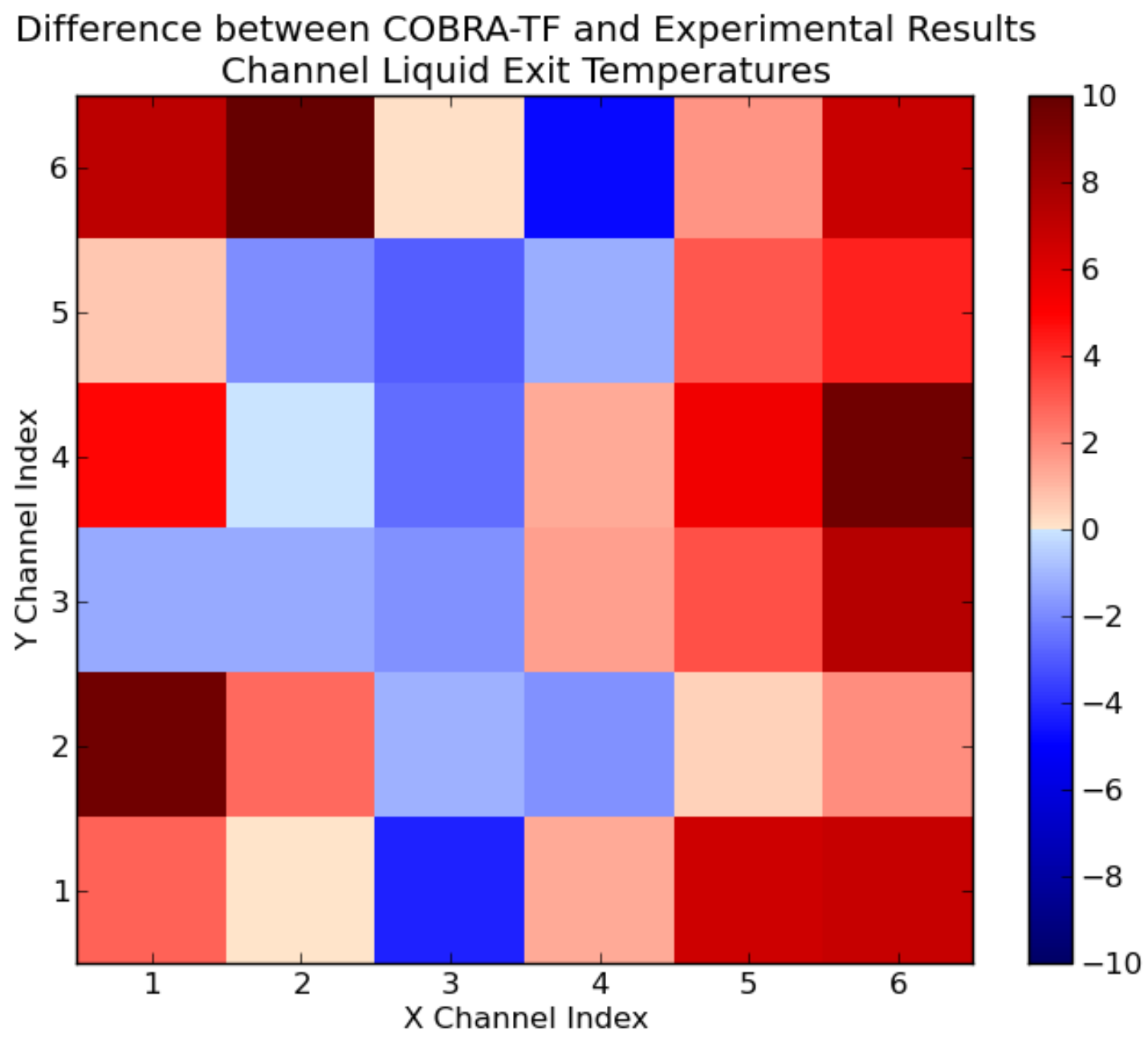

Figure 6.1: Average difference between CTF predicted channel exit temperatures and experimental values for all tests in CE 5x5 Series 74 


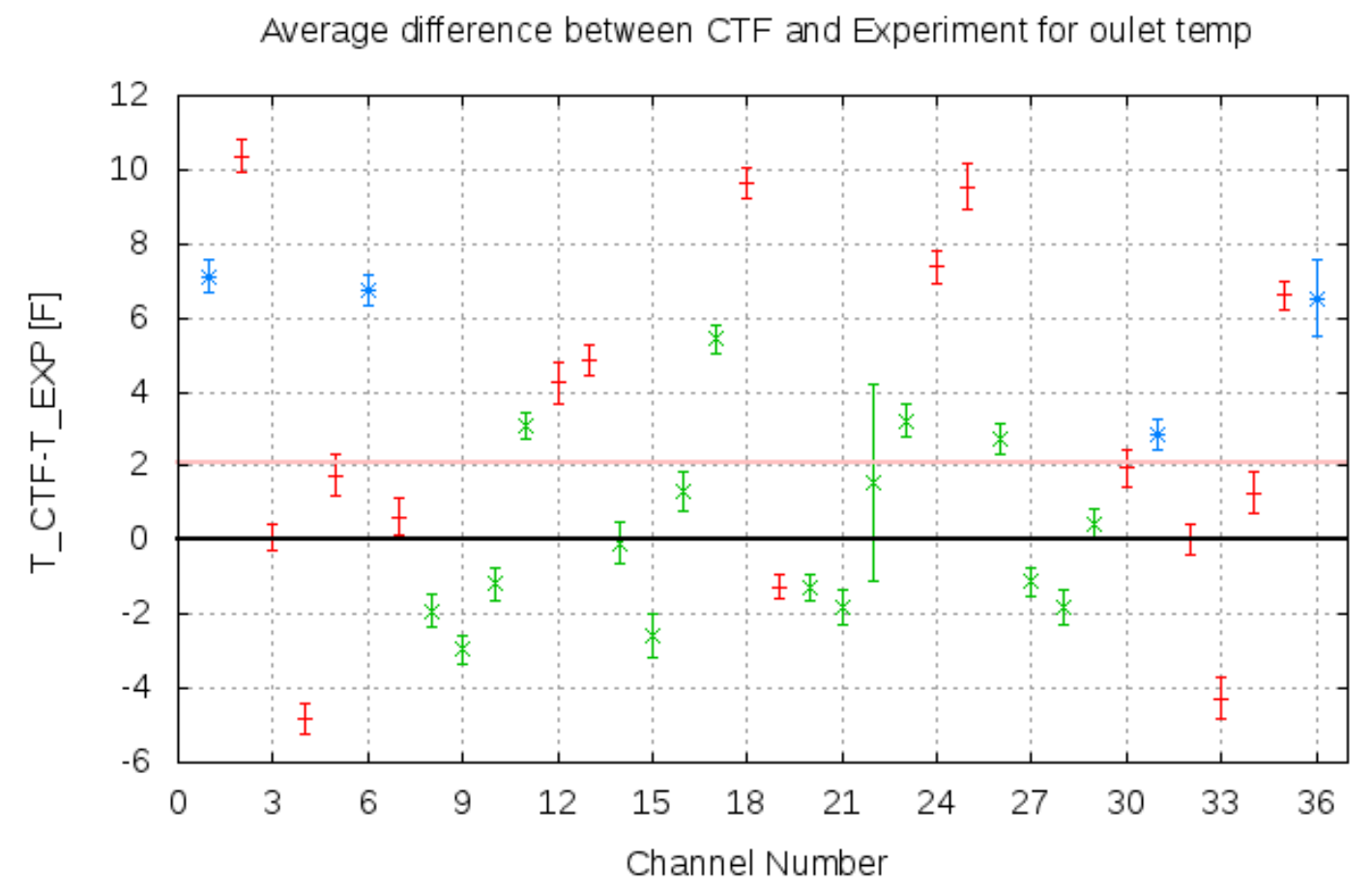

Side Channels

Corner Channels $\longmapsto$ Inner Channels Average Discrepancy

Figure 6.2: Average difference between CTF predicted channel exit temperatures and experimental values with measurement error and mean discrepancy 

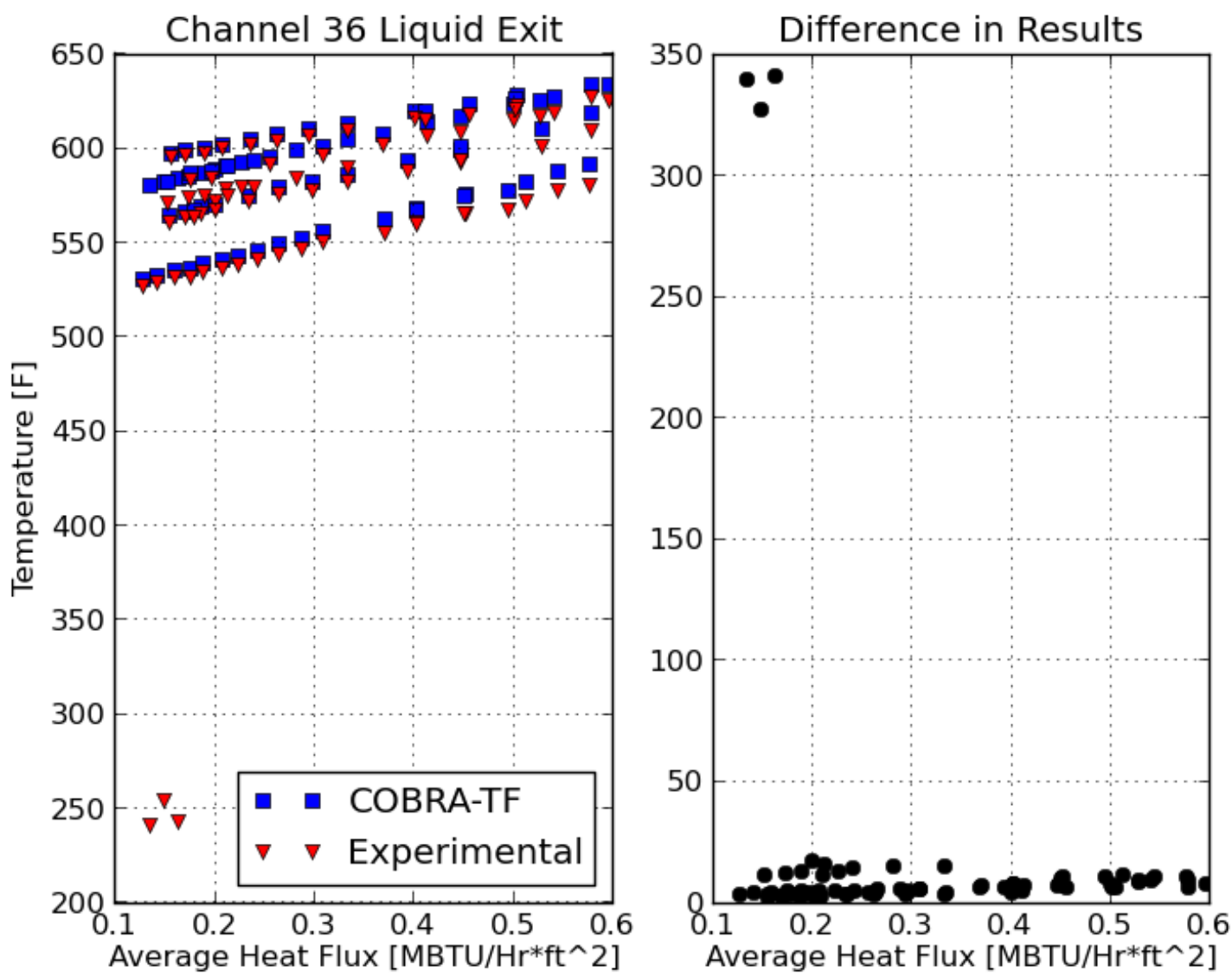

Figure 6.3: Predicted and measured outlet temperatures for Channel 36 in all CE 5x5 Series 74 cases 


\subsubsection{Kumamoto University $2 \times 3$}

Results are shown as the nondimensional mixing rate, $W_{i j}^{\prime} / \mu$, vs. the two-channel Reynolds number. The two-channel Reynolds number is calculated as follows:

$$
R e_{i j}=\frac{\rho u_{i j} D_{e, i j}}{\mu}
$$

Where the average two-channel velocity, $u_{i j}$, is an area weighted average of the velocities in the two adjacent channels, $i$ and $j$. The hydraulic diameter, $D_{e, i j}$, is the hydraulic diameter of the two-channel system, $\rho$ is density, and $\mu$ is dynamic viscosity. This is the way in which the author presents mixing results. A mixing rate is given for each unique channel connection, which includes: 1) inner-to-inner, 2) side-to-inner, and 3) side-to-side for each of the four single phase tests. Figure 6.4 shows the CTF results compared with the experimental results using the Rogers and Rosehart correlation [2] to predict single-phase mixing. The Blasius friction factor correlation is used in this case.

Two types of data points are shown in the plot: pluses represent the CTF predictions and circles represent the experimental measurements. There are three colors of the data points: red represents the inner-to-inner connection, green represents the side-to-side connection, and blue represents the side-to-inner connection. Ideally, a "plus" and a "circle" data point should sit in a vertical column; this would mean that the $i j$ Reynolds number of CTF matches the experimental value exactly. Looking at the figure, it is evident this is not the case. The CTFpredicted Reynolds number tends to be higher than its experimental counterpart in every case.

Likely, there are some differences in steam properties and inlet mass flow rate that lead to this discrepancy. However, it is evident that there is a near-linear trend for nondimensional mixing with respect to Reynolds number that we can use for comparison. The results of Figure 6.4 indicates that the Rogers and Rosehart correlation over-predicts the mixing rate observed in this facility substantially.

The study is re-run with a user-set, constant single-phase mixing coefficient of 0.004 . A mixing-coefficient optimization study done using the CE $5 \times 5$ facility found that a value of 0.0044 was optimum for that configuration [18], so 0.004 is considered to be a lower bounding value. Figure 6.5 shows the results of changing the mixing coefficient to 0.004 .

Results indicate that this mixing coefficient underpredicts the mixing in the facility. As it turns out, a mixing coefficient of about 0.007 tends to lead to the best agreement, as shown in Figure 6.6. The choice of friction factor correlation has little impact on the predicted mixing rates. Figure 6.7 shows the results using $\beta=0.004$ with the friction factor correlation set to the CTF correlation instead of the Blasius correlation.

It is important to note that the mixing coefficient is simply a tuning parameter that will be dependent on the actual geometry of the facility being modeled. This facility is a square lattice, but the geometry is much larger than typical PWR or BWR rod-lattice geometry. This study is useful for showing that $\mathrm{CTF}$ is capable of predicting the correct mixing rate if $\beta$ is tuned correctly to the facility. Furthermore, it offers a range of values from which to select the mixing coefficient. 


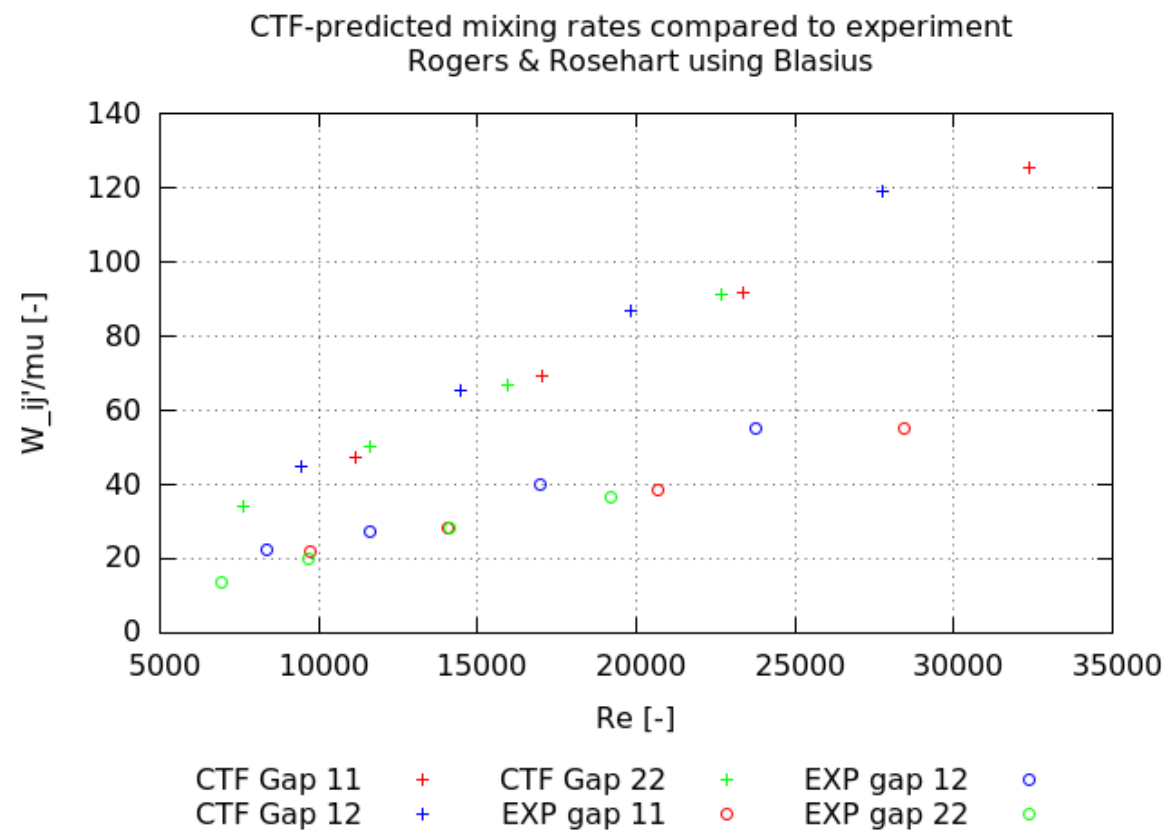

Figure 6.4: Comparison of CTF-predicted mixing rates and experimental measured rates from $2 \times 3$ facility using Rogers and Rosehart for $\beta$ and the Blasius friction correlation.

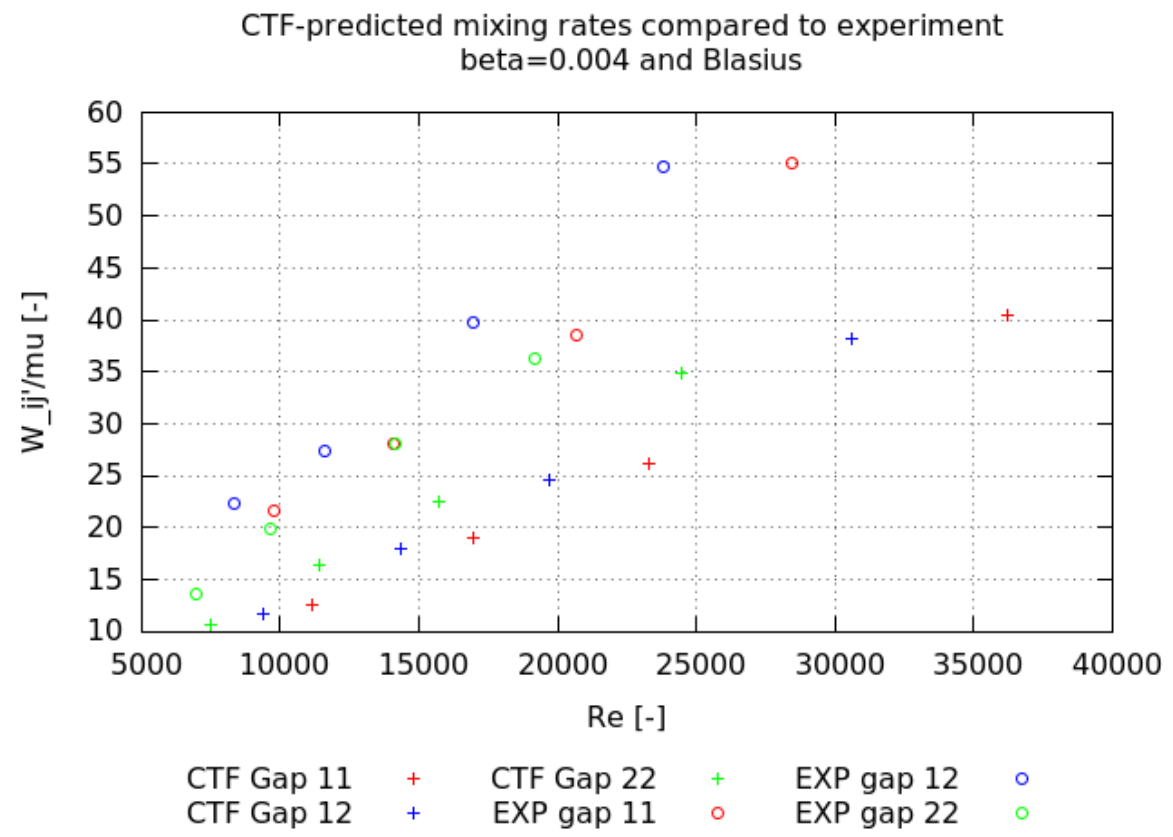

Figure 6.5: Comparison of CTF-predicted mixing rates and experimental measured rates from $2 \times 3$ facility using $\beta=0.004$ and the Blasius friction correlation. 


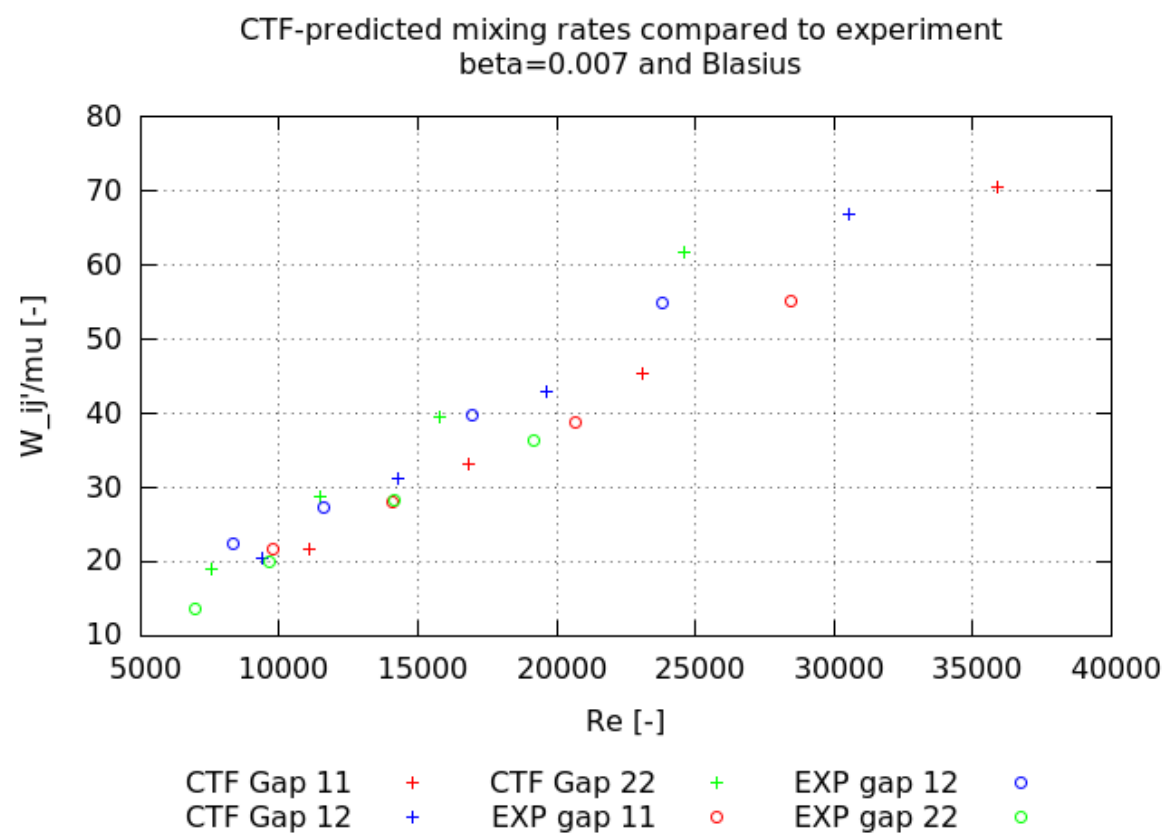

Figure 6.6: Comparison of CTF-predicted mixing rates and experimental measured rates from $2 \times 3$ facility using $\beta=0.007$ and the Blasius friction correlation.

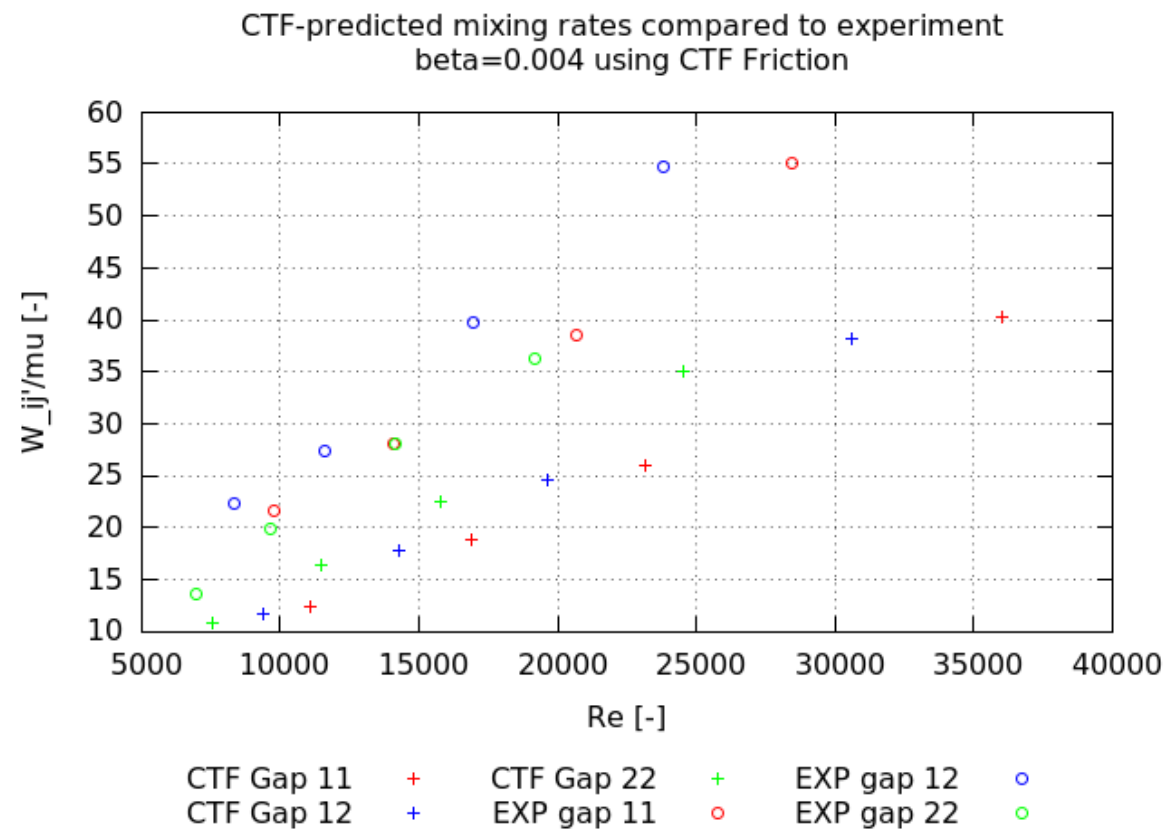

Figure 6.7: Comparison of CTF-predicted mixing rates and experimental measured rates from $2 \times 3$ facility using $\beta=0.007$ and the CTF friction correlation. 


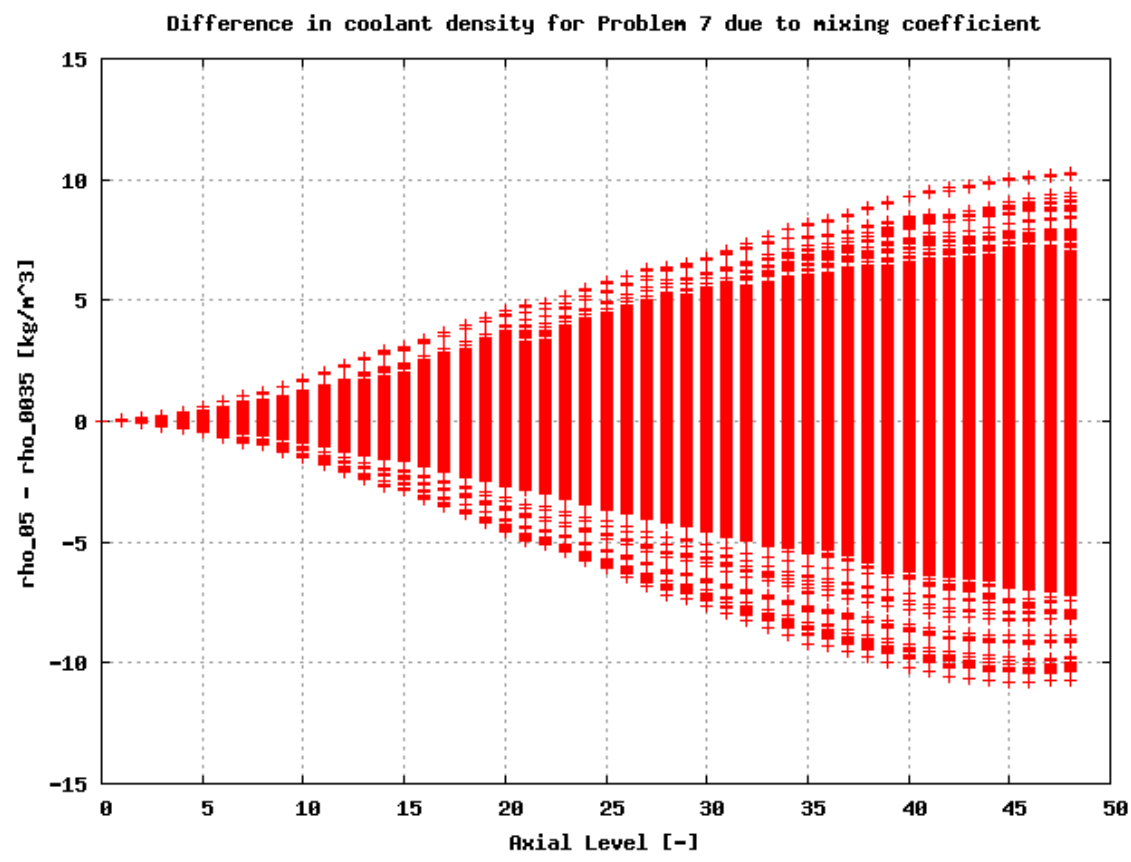

Figure 6.8: Variation of local liquid density in CTF simulation of Watts Bar Unit 1 when $\beta$ is changed from 0.0035 to 0.05 .

The physical relevance of the mixing rate is not immediately obvious. It is better to observe the impact of the term on simulation parameters that affect the solution. The CASL Problem 7 challenge problem (quarter symmetry model of Watts Bar Unit 1) is modeled using a power distribution from a coupled MPACT/CTF solution of the facility. The mixing coefficient is changed from 0.0035 to 0.05 , with 0.05 being much greater than the value predicted by Rogers and Rosehart. The impact of changing this parameter on local predicted liquid density is shown in Figure 6.8. The results are presented as density in a cell when $\beta$ is 0.05 minus density in the cell when $\beta$ is 0.0035 . This calculation is made in each of the roughly 500,000 computational cells of the model and presented in the figure. The results show that differences increase to a maximum at the outlet of the facility and reach as much as $0.01 \mathrm{~g} \mathrm{~cm}^{-3}$, which will have a small, but noticeable impact on reactivity in those locations.

\subsubsection{RPI $2 \times 2$}

In this step of the validation, we are concerned only with correctly predicting the single-phase flow distribution. Therefore, only the two single-phase tests are modeled (Tests 1 and 2). The ideal flow distribution is determined similar to how it is in Section 5.2.2.1; the momentum equation is used to relate velocity in two individual subchannels, and the mass conservation equation is used to link all channels together. In this case, we have two unique momentum equations and one mass equation with three unknown variables. The coefficients for the CTF friction correlation are used in the momentum equation, leading to an exponent of $2 / 3$ on the ratio of hydraulic diameters. 


$$
\begin{aligned}
\frac{u_{\text {corner }}}{u_{\text {side }}} & =\left(\frac{D h_{\text {corner }}}{D h_{\text {side }}}\right)^{2 / 3} \\
\frac{u_{\text {corner }}}{u_{\text {inner }}} & =\left(\frac{D h_{\text {corner }}}{D h_{\text {inner }}}\right)^{2 / 3} \\
\dot{m}_{\text {tot }} & =4 \rho u_{\text {corner }} A_{\text {corner }}+4 \rho u_{\text {side }} A_{\text {side }}+\rho u_{\text {inner }} A_{\text {inner }}
\end{aligned}
$$

Solving this system of equations gives an ideal flow split for each individual channel type:

$$
\begin{gathered}
\dot{m}_{\text {corner }}=\frac{\dot{m}_{\text {tot }}}{C}\left(\frac{D h_{\text {corner }}}{D h_{\text {inner }}}\right)^{2 / 3}\left(\frac{A_{\text {corner }}}{A_{\text {inner }}}\right) \\
\dot{m}_{\text {side }}=\frac{\dot{m}_{\text {tot }}}{C}\left(\frac{D h_{\text {side }}}{D h_{\text {inner }}}\right)^{2 / 3}\left(\frac{A_{\text {side }}}{A_{\text {inner }}}\right) \\
\dot{m}_{\text {inner }}=\frac{\dot{m}_{\text {tot }}}{C}, \text { where } \\
C=4\left(\frac{D h_{\text {corner }}}{D h_{\text {inner }}}\right)^{2 / 3} \frac{A_{\text {corner }}}{A_{\text {inner }}}+4\left(\frac{D h_{\text {side }}}{D h_{\text {inner }}}\right)^{2 / 3} \frac{A_{\text {side }}}{A_{\text {inner }}}+1 .
\end{gathered}
$$

Each single-phase case is first run with turbulent mixing disabled, so as to see that CTF predicts the correct single-phase flow distribution. Results show that CTF does predict the correct flow distribution; however, not within the axial length of the test section, which is $1 \mathrm{~m}$. The CTF model is extended to $7 \mathrm{~m}$ to show that the correct flow split is eventually achieved. Figures 6.9 and 6.10 show the results of running CTF with no turbulent mixing for Case 1 and 2 of the $2 \times 2$ facility, respectively.

The channel mass flux results are normalized as shown in Equation 5.16. The figures show four important pieces of information:

1. The CTF normalized channel mass fluxes are shown for corner, side, and inner type channels (red, blue, and green) using the solid lines,

2. The analytical solution for the flow split (obtained using Equations 6.5, 6.6, and 6.7) is shown with the three horizontal dashed lines using the same color scheme to denote channel types,

3. The experimental measurements are shown with the dot-dash lines using the same color scheme for denoting channel type, and

4. The shaded regions show the maximum experimental measurement uncertainty for channel mass flux (5\%), as quoted in the $2 \times 2$ technical report [14].

The figures shows that the trend for flow to migrate into the lower resistance inner channel and out of the higher resistance corner channel is correctly predicted. The CTF channel flows 


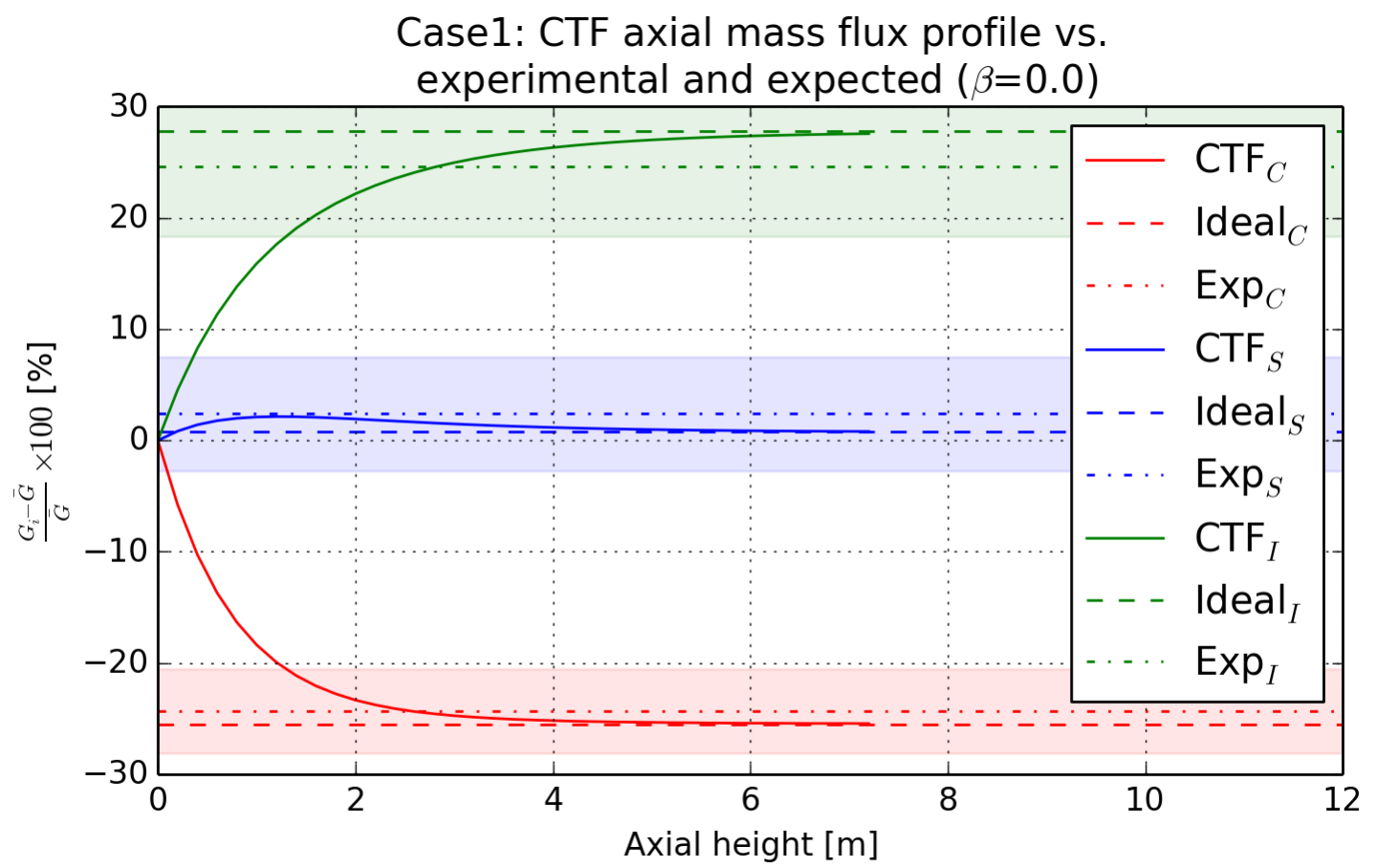

Figure 6.9: CTF-predicted flow split (no mixing) for $2 \times 2$ Case 1 single-phase test compared with experimental values and ideal flow split.

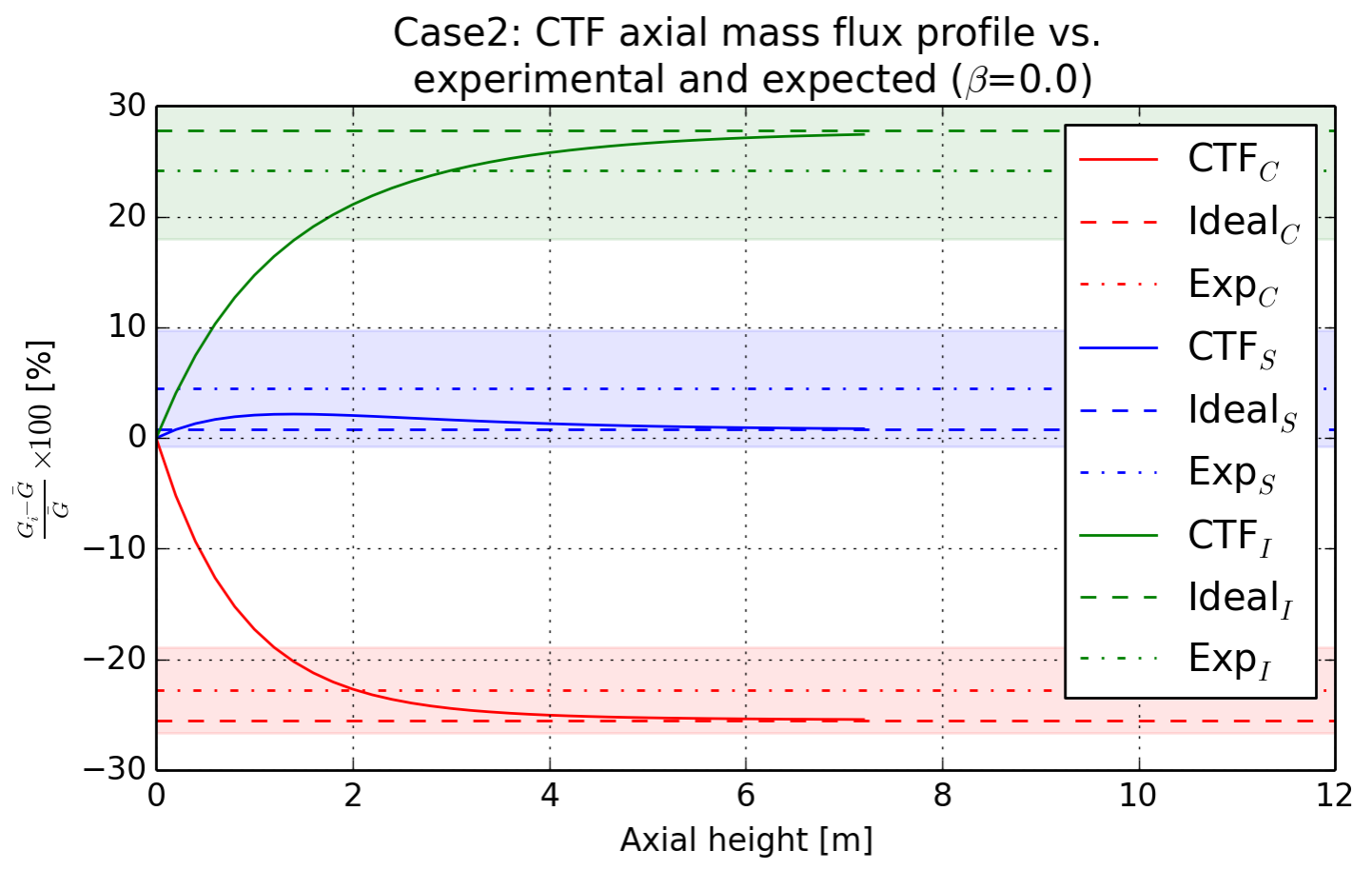

Figure 6.10: CTF-predicted flow split (no mixing) for $2 \times 2$ Case 2 single-phase test compared with experimental values and ideal flow split. 
hit the expected values at about $6 \mathrm{~m}$. The experimental results are not exactly the same as the theoretical values since turbulent mixing drives momentum from the higher velocity inner channel back to the corner and side channels. However, note that the experimental results are obtained within $1 \mathrm{~m}$ of test section length.

Figures 6.11 and 6.12 show the same results with turbulent mixing enabled in CTF and the single-phase turbulent mixing coefficient, $\beta$, set to 0.007 (the ideal value discovered in Section $6.1 .2)$.

Enabling turbulent mixing leads to a new mechanical equilibrium point in CTF. The inner channel flow does not go as high and corner channel flow does not go as low. The results seem to indicate that the CTF mixing coefficient may be slightly too high for this experimental facility. The CTF inner channel mass flux is lower than the experimental inner channel measurement and the CTF corner channel mass flux is higher the than the experimental corner measurement. The corner channel prediction is just outside of the measurement uncertainty bands.

Adjusting the mixing coefficient down to $\beta=0.0035$ leads to a more favorable prediction of the flow split, as shown in Figures 6.13 and 6.14 .

Similar to the cases without mixing, the flow takes about 5-6 $\mathrm{m}$ to reach equilibrium distribution in the CTF model. Since Case 2 has a mass flux that is twice that of Case 1, it appears that the magnitude of flow has no significant effect on the distance to reach equilibrium flow distribution. 


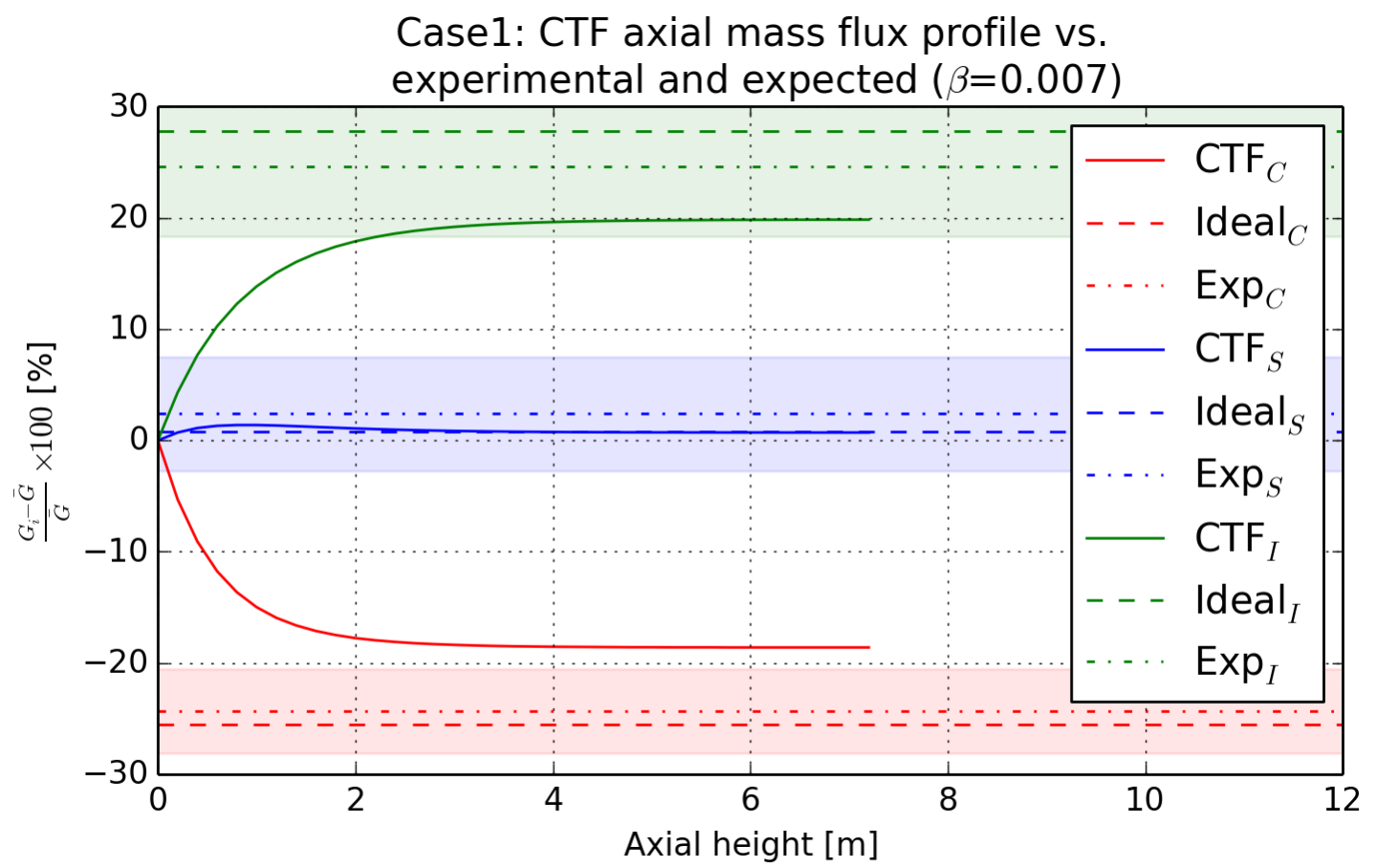

Figure 6.11: CTF-predicted flow split (with $\beta$ set to 0.007 ) for $2 \times 2$ Case 1 single-phase test compared with experimental values and ideal flow split.

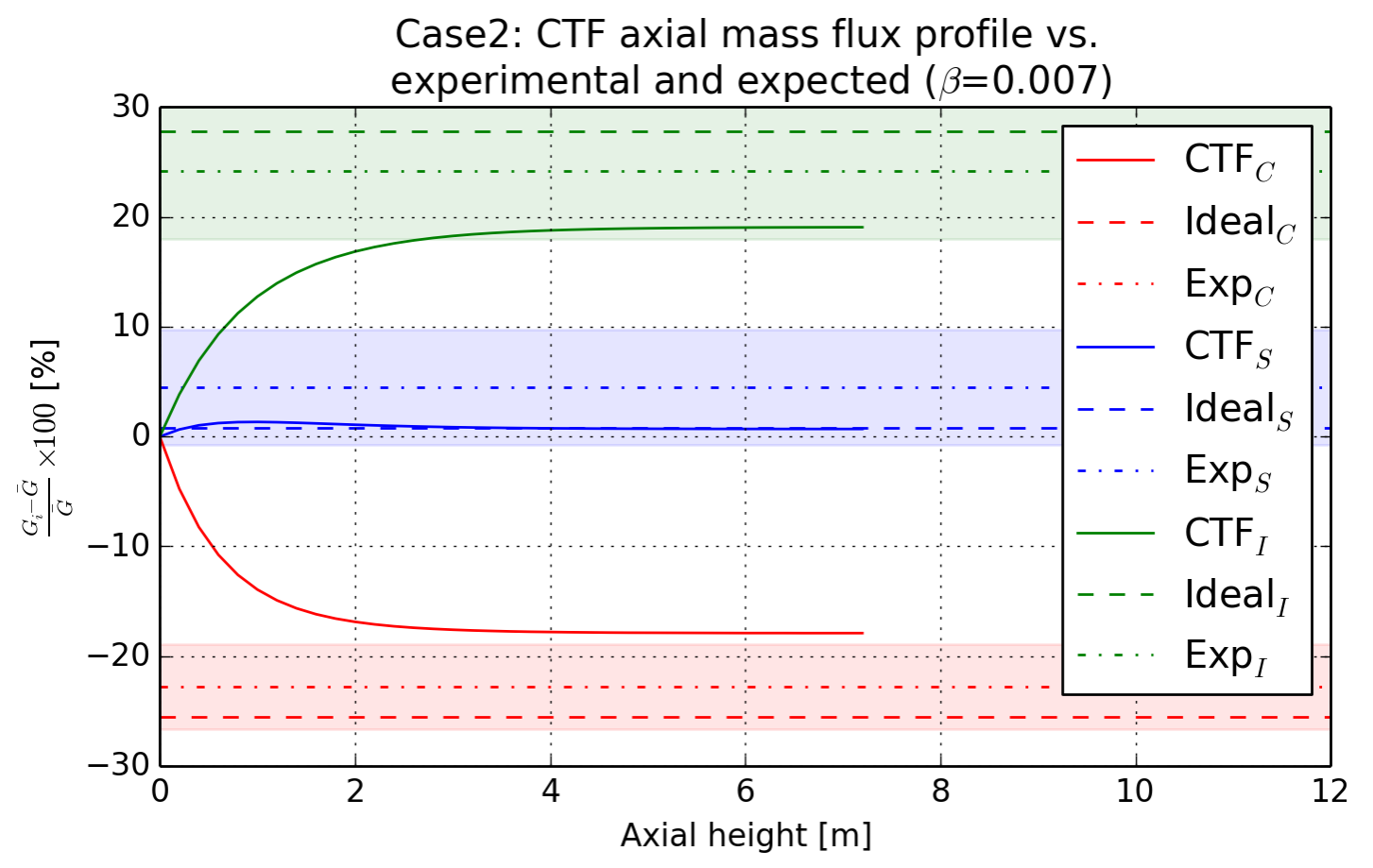

Figure 6.12: CTF-predicted flow split (with $\beta$ set to 0.007 ) for $2 \times 2$ Case 2 single-phase test compared with experimental values and ideal flow split. 


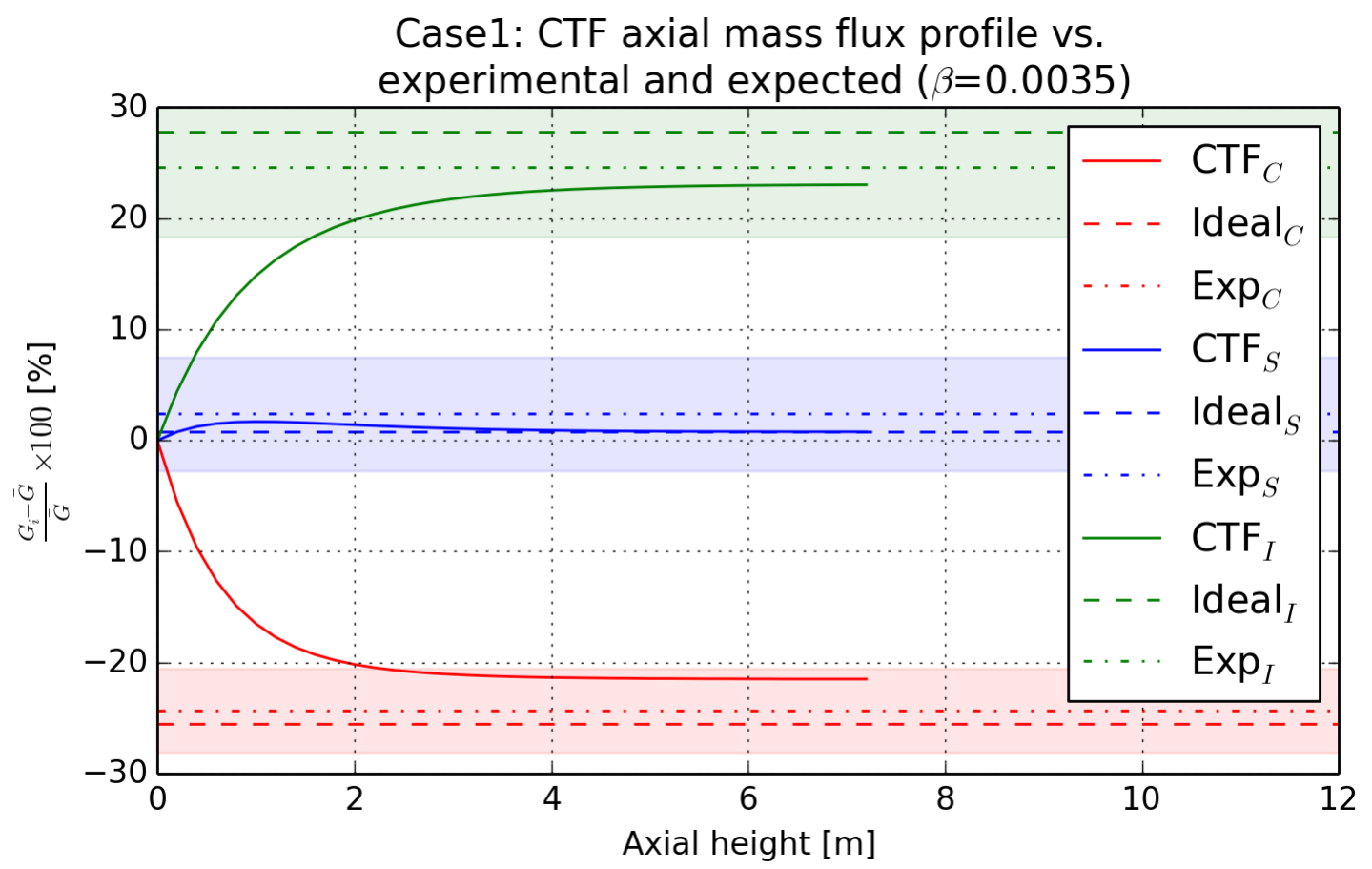

Figure 6.13: CTF-predicted flow split (with $\beta=0.0035$ ) for $2 \times 2$ Case 1 single-phase test compared with experimental values and ideal flow split.

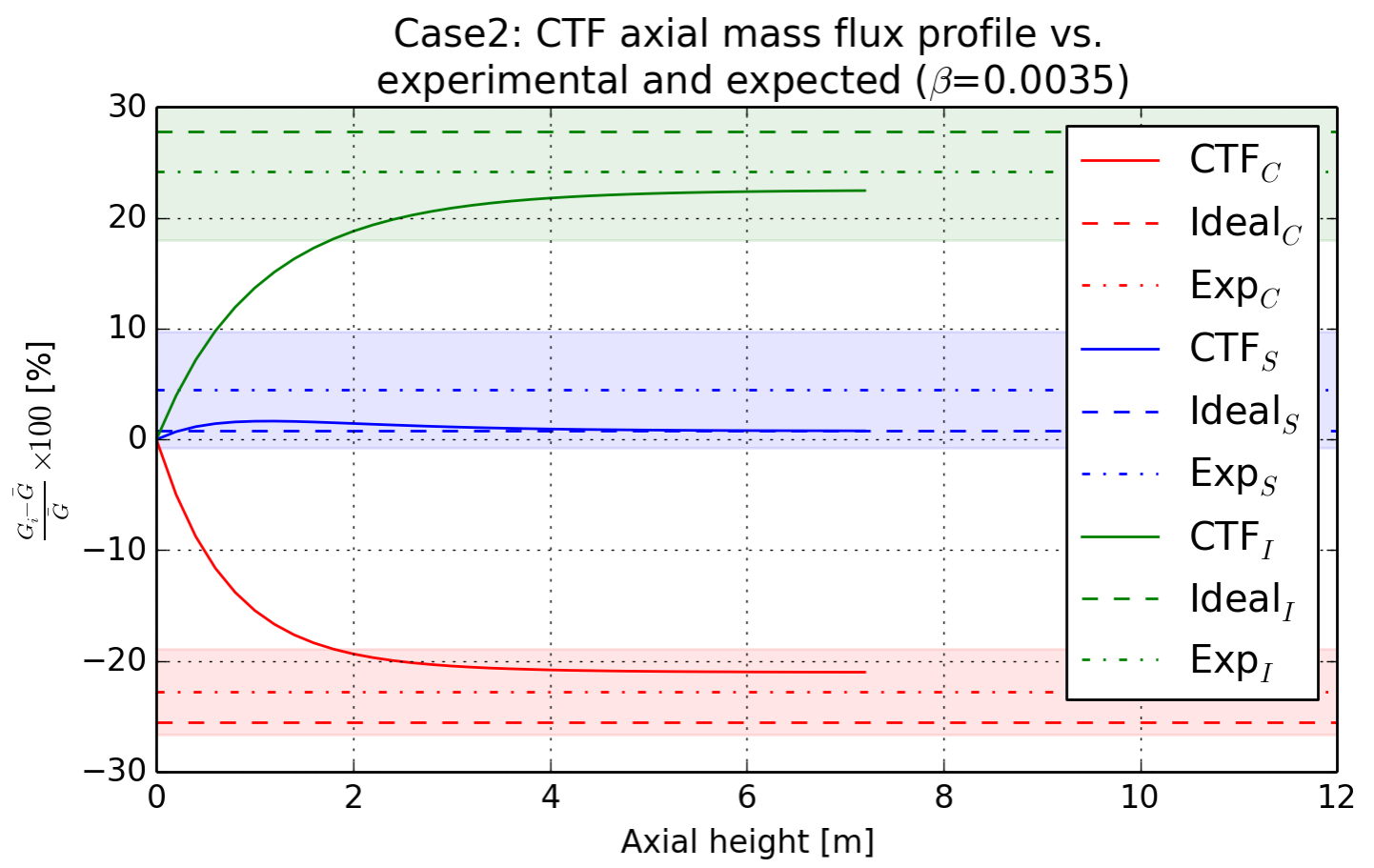

Figure 6.14: CTF-predicted flow split (with $\beta=0.0035$ ) for $2 \times 2$ Case 2 single-phase test compared with experimental values and ideal flow split. 


\subsubsection{GE $3 \times 3$}

Because this section of the study is reviewing the ability to predict correct single-phase flow distribution, only the four single-phase cases $(1 \mathrm{~B}, 1 \mathrm{C}, 1 \mathrm{D}$, and $1 \mathrm{E})$ are run. Considering the difficulty CTF exhibited in correctly predicting the flow split for the $2 \times 2$ facility, it is prudent to assess the flow-distribution prediction for the GE $3 \times 3$ case before analyzing the two-phase void drift cases.

The cases are first run without spacer grids and with turbulent mixing disabled. The ideal flow split is calculated using the same approach as used in Section 5.2.2.1. Again, flow results are displayed as normalized values, the ideal flow split is shown with dashed horizontal lines, and the measured results are shown with dot-dash lines. The measurement uncertainty is shown as a colored, shaded region around the measurement line. Figures 6.15-6.18 show the result of these predictions for the four single-phase cases with no turbulent mixing or form losses.

Similar to the $2 \times 2$ facility tests, CTF predicts the correct flow rate distribution; however, it is interesting to note that the equilibrium distribution is achieved in a much shorter length of the facility. The flow distribution is nearly in equilibrium at the $1.8 \mathrm{~m}$ location, which is the exit of the facility; whereas, the $2 \times 2$ facility took nearly $5 \mathrm{~m}$ to reach equilibrium.

Adding the grids and turbulent mixing produces the results shown in Figures 6.19-6.22. The channel mass fluxes are closer to the bundle-average value as a result of the turbulent mixing model being enabled. The addition of the grids results in a "choppy" appearance of the axial mass flux distributions. Because the grid form loss coefficient is the same for all channels, the grids have an "equalizing" effect, pushing inner channel flow back into the side and corner channels. The redistribution is driven by the fact that the higher-velocity inner channel flow experiences a greater pressure loss than the lower-velocity corner channel at the grid location. The imbalance in flow losses causes flow to migrate from the higher resistance inner channel to the lower resistance corner channel. The redistribution lasts for a short period downstream of the grid loss and soon reverses so that flow moves back into the inner channel.

All single-phase predicted outlet mass fluxes are compared with their measured counterparts in Figure 6.23. Each color represents a unique channel type: red for corner, blue for side, and green for inner. Inner- and side-predicted mas fluxes match experimental values closely, having rRMS (Equation 1.5) values that are close to experimental measurement uncertainty. Corner results vary from experimental values by a much larger degree.

The excellent agreement between CTF and the experiment found in this single-phase study adds more credibility to the two-phase void drift study performed in Section 8.1.1. However, it is noted that the turbulent mixing model in this study is different from the one used in the Validation Manual. The original GE $3 \times 3$ study was done using the Rogers \& Rosehart correlation to calculate the mixing coefficient. This choice is further investigated in this section and the two-phase void drift modeling section.

It has been shown in Section 6.1.2 that the Rogers \& Rosehart model tends to exaggerate the mixing in the system. The single-phase cases are re-run using the Rogers \& Rosehart model for $\beta$ and measured versus predicted exit mass fluxes are shown in Figure 6.24. Side and inner results are largely unaffected, but the corner channel experiences a more significant drop in accuracy. Overall rRMS increases by two percentage points. The average error (Equation 1.4) increases from $3.8 \%$ to $4.8 \%$. 


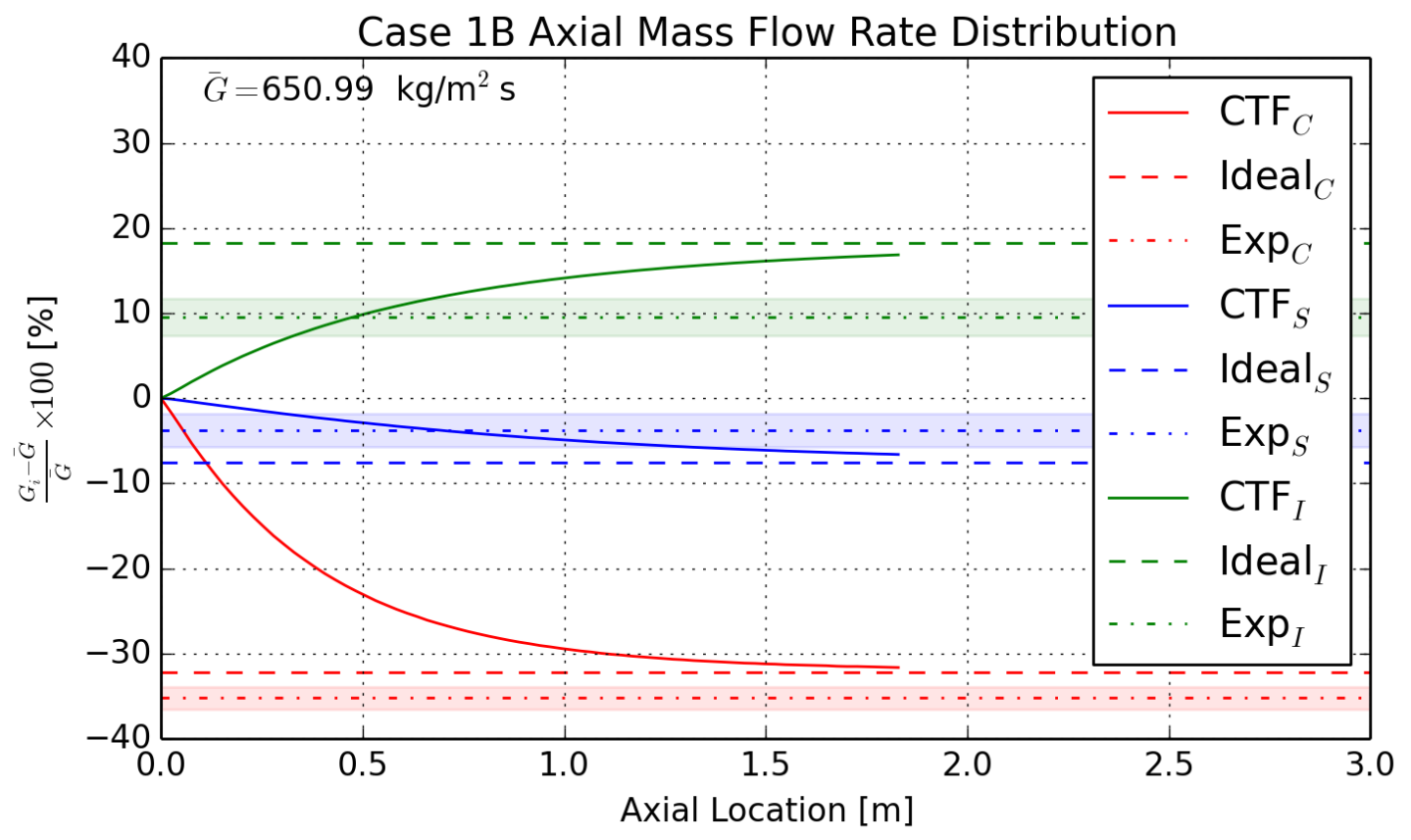

Figure 6.15: CTF-predicted flow split compared with ideal and measured results for GE $3 \times 3$ Case 1B (no mixing or spacer grids in model).

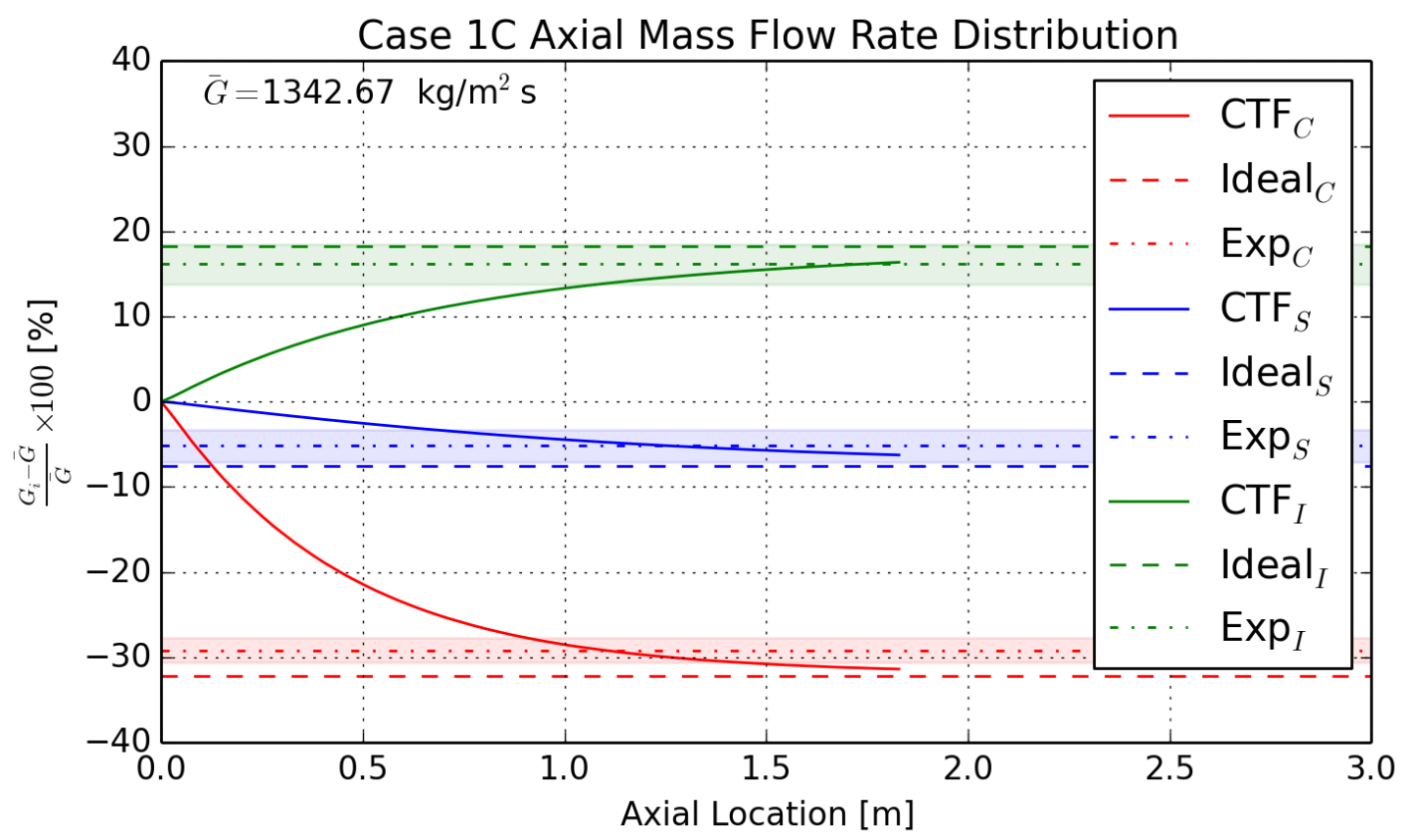

Figure 6.16: CTF-predicted flow split compared with ideal and measured results for GE $3 \times 3$ Case $1 \mathrm{C}$ (no mixing or spacer grids in model). 


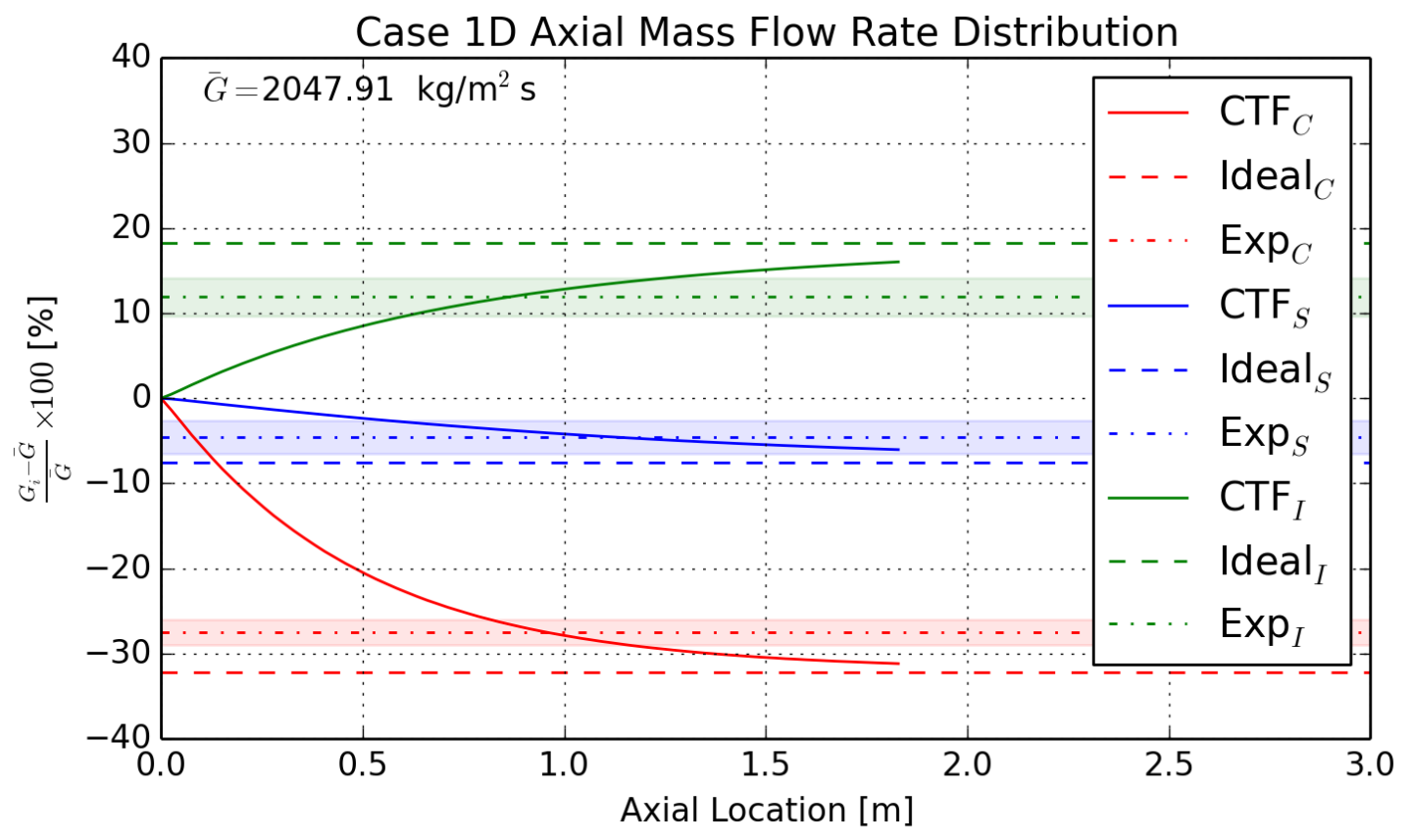

Figure 6.17: CTF-predicted flow split compared with ideal and measured results for GE $3 \times 3$ Case 1D (no mixing or spacer grids in model).

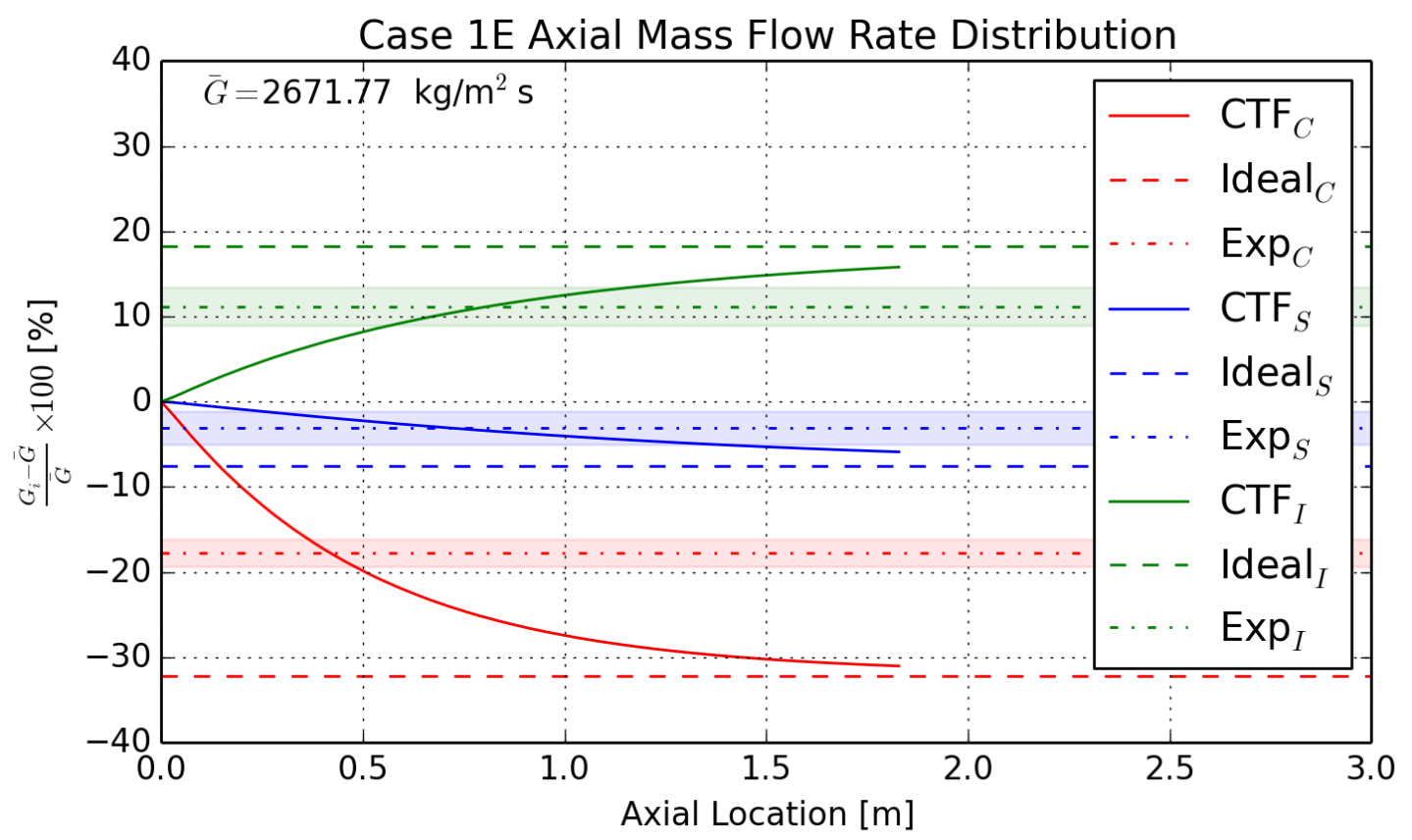

Figure 6.18: CTF-predicted flow split compared with ideal and measured results for GE $3 \times 3$ Case $1 \mathrm{E}$ (no mixing or spacer grids in model). 


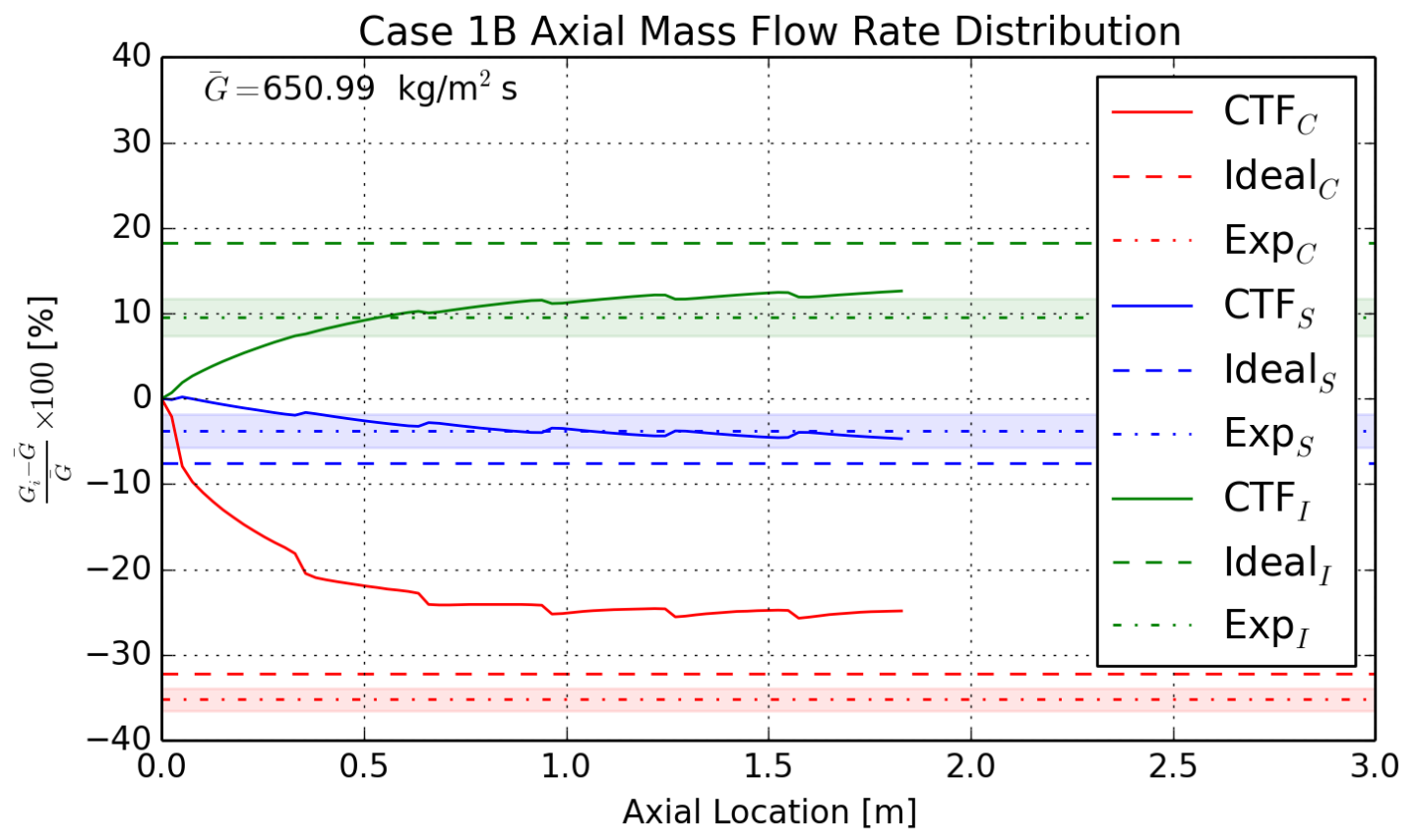

Figure 6.19: CTF-predicted flow split compared with ideal and measured results for GE $3 \times 3$ Case 1B (with $\beta=0.007$ ).

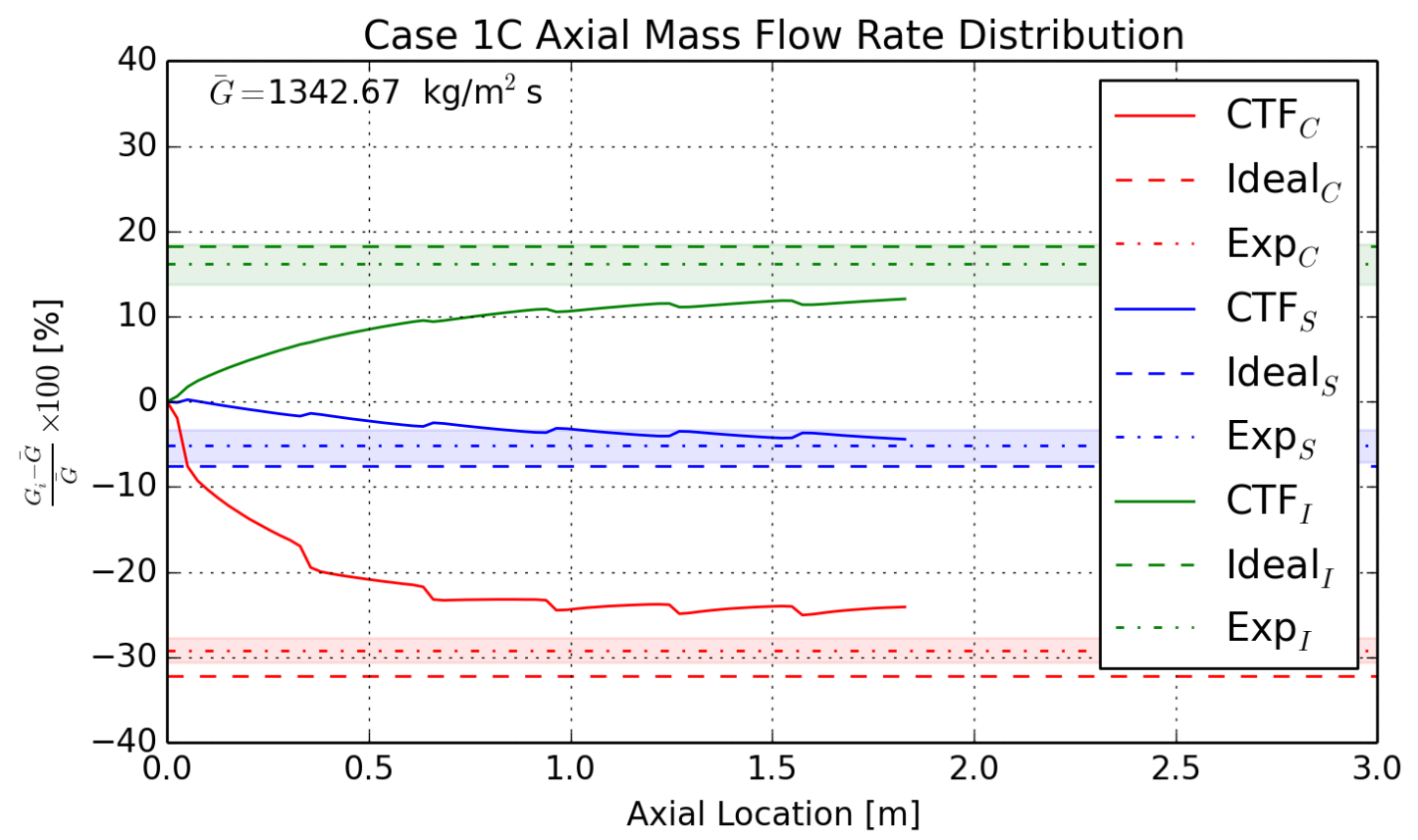

Figure 6.20: CTF-predicted flow split compared with ideal and measured results for GE $3 \times 3$ Case $1 \mathrm{C}$ (with $\beta=0.007$ ). 


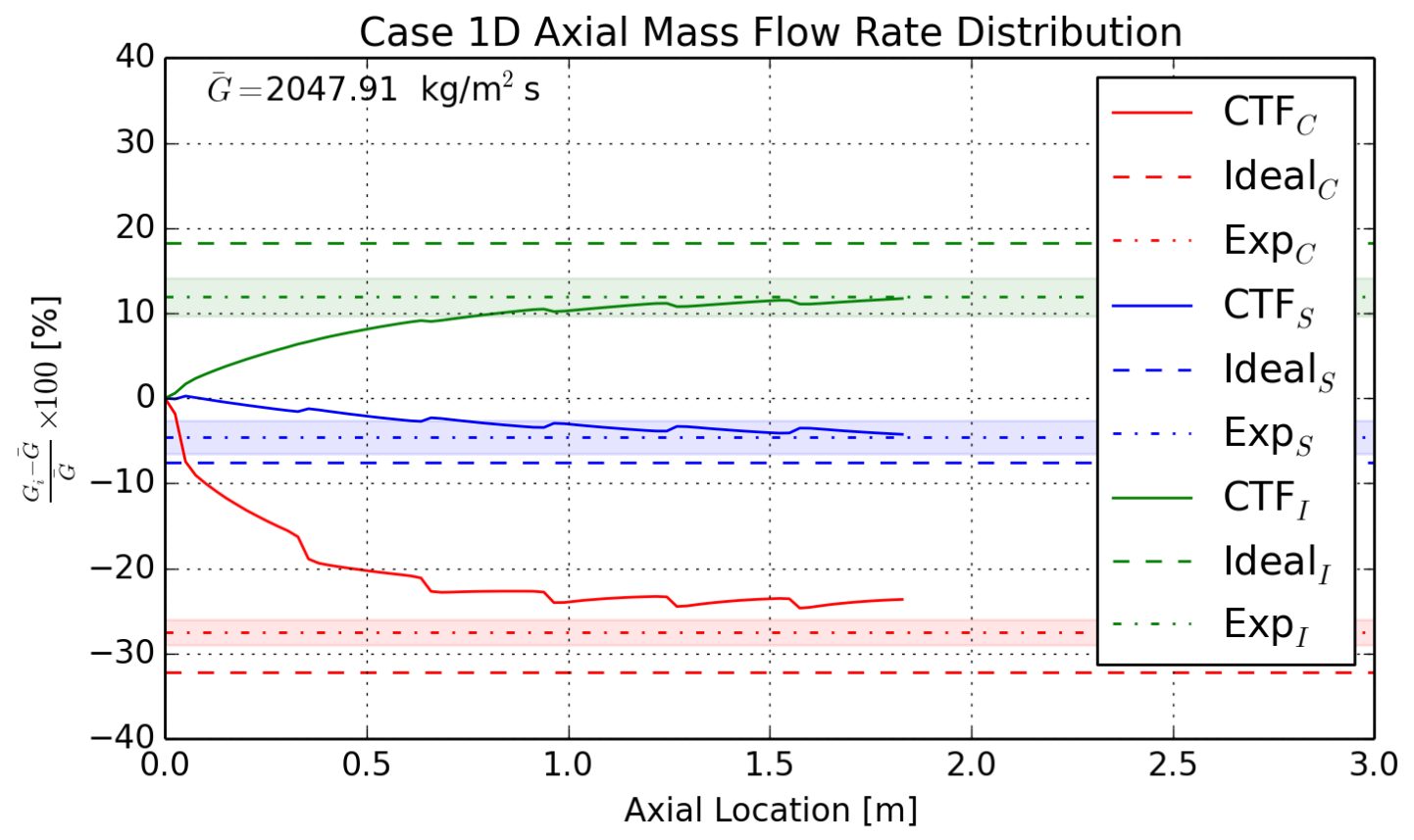

Figure 6.21: CTF-predicted flow split compared with ideal and measured results for GE $3 \times 3$ Case 1D (with $\beta=0.007$ ).

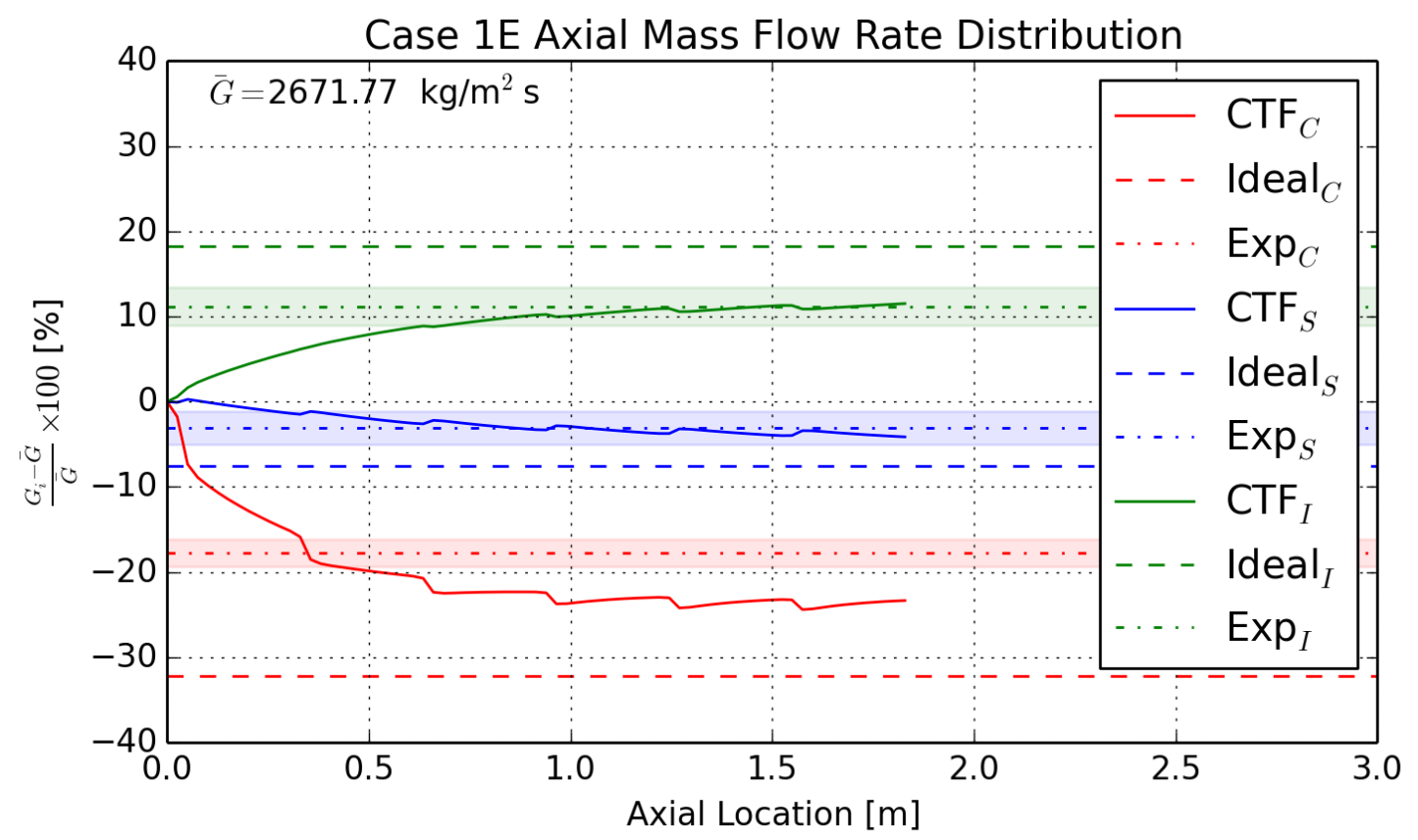

Figure 6.22: CTF-predicted flow split compared with ideal and measured results for GE $3 \times 3$ Case $1 \mathrm{E}$ (with $\beta=0.007$ ). 


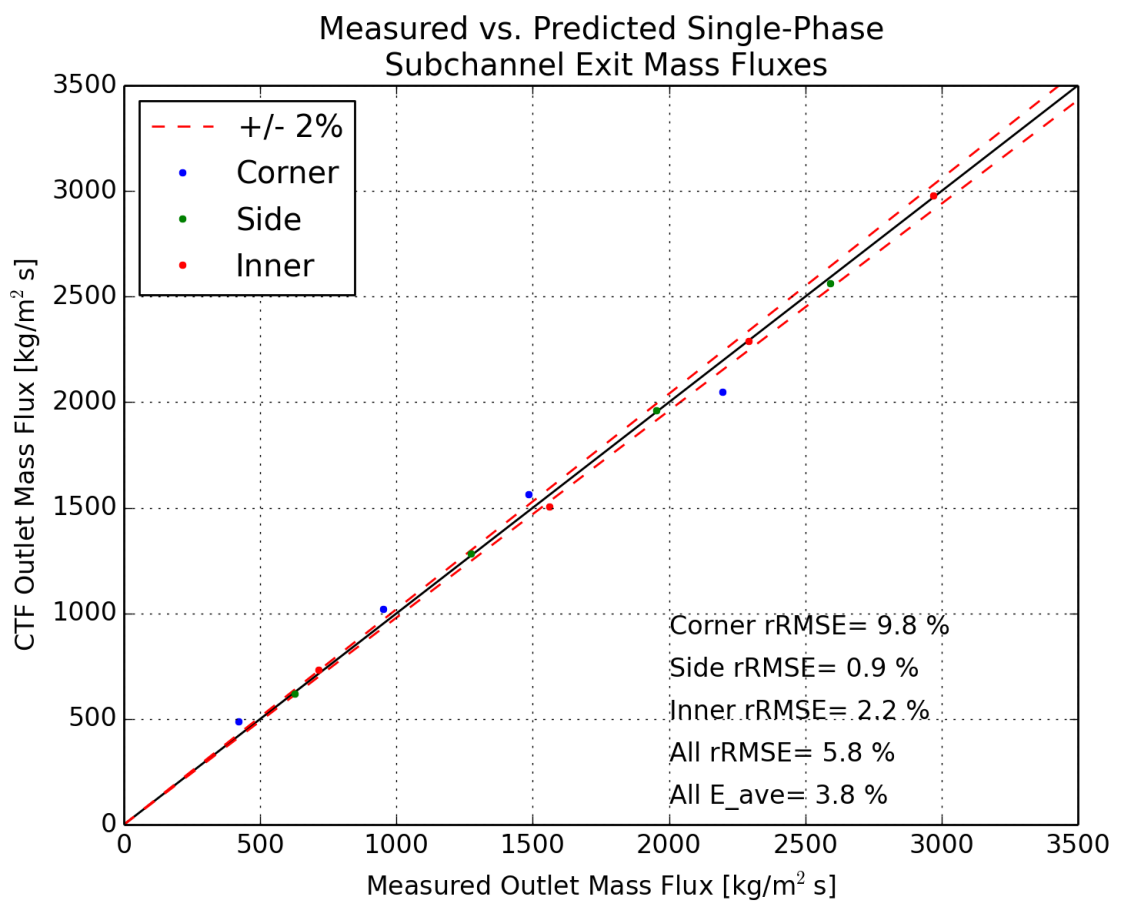

Figure 6.23: Comparison of predicted and measured exit mass flux for GE $3 \times 3$ single-phase cases using $\beta=0.007$.

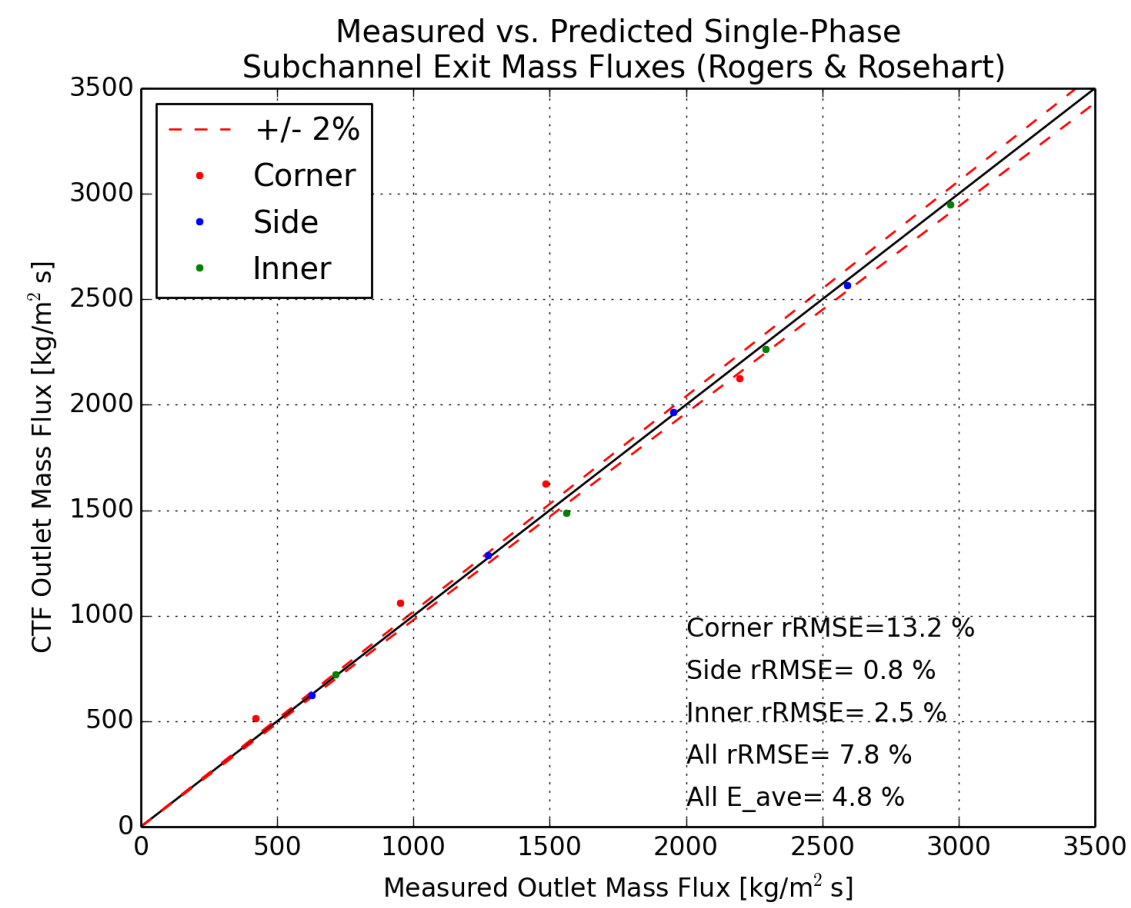

Figure 6.24: Comparison of predicted and measured exit mass flux for GE $3 \times 3$ single-phase cases using Rogers \& Rosehart to calculate $\beta$. 


\subsection{Verification}

\subsubsection{Single-Phase Two-Channel}

Problem Description The problem consists of two channels connected by a gap. Because the CTF model for turbulent mixing is gradient-driven, it is necessary to make a gradient in either energy or momentum. Because there is no net transfer of mass due to turbulent mixing in single phase, unheated flows, it is not possible to analyze mass transfer in this case. Forming an analytical solution requires us to form and solve the relevant governing equations for the system.

If we choose to look at turbulent mixing of momentum, we will need to set velocity of one channel higher than the other. The result will be migration of velocity due to pressure-driven directed cross-flow (due to higher frictional pressure drop in the high velocity channel) as well as turbulent mixing of the momentum. We wish to verify that the turbulent mixing model works as expected without interference from other effects. We can disable the friction model to stop the pressure-driven directed cross-flow. However, because the axial velocity profile will not be constant in the channel, the convective terms of the momentum equation cannot be eliminated, which requires a complicated solution of the equations.

The energy equation can be solved much more easily as long as we disable the temperaturedependent density in CTF. With this disabled, the velocity profile will be constant, as the turbulent mixing model for energy does not actually move mass from one channel to another; it captures the effect of mixing on the migration of energy from one channel to the other.

This problem is a modification of Example 6-1 in Todreas and Kazimi Volume II [19]. The problem in the textbook uses the concept of tracer dyes to demonstrate mixing. In place of looking at mixing of a dye, the mixing of enthalpy is observed in this problem.

The design of the system is shown in Figure 6.25. The geometry of the two channels is identical in this case, which should eliminate any pressure-driven directed cross-flow. Channel geometry is based on typical PWR rod-lattice geometry. To activate the turbulent-mixing model, the temperature of one channel is raised $10^{\circ} \mathrm{C}$ over the second channel. The "vuq_param.txt" file is used to set a constant liquid density in the system.

For this case, we can set up an energy equation for each channel. The CTF energy equation is as follows:

$$
\begin{gathered}
\frac{\partial}{\partial t}\left(\alpha_{k} \rho_{k} h_{k}\right)+\frac{\partial}{\partial x}\left(\alpha_{k} \rho_{k} h_{k} u_{k}\right)+\frac{\partial}{\partial y}\left(\alpha_{k} \rho_{k} h_{k} v_{k}\right)+\frac{\partial}{\partial z}\left(\alpha_{k} \rho_{k} h_{k} w_{k}\right)= \\
\Sigma_{\text {gap }=1}^{N G A P} q_{k, \text { gap }}^{T^{\prime \prime \prime}}+\Gamma^{\prime \prime \prime} h+q_{w k}^{\prime \prime \prime}+\alpha_{k} \frac{\partial P}{\partial t}
\end{gathered}
$$

The left-hand side terms include: 1) time-change of energy, 2) axial (x) advection of energy, 3 ) lateral ( $y$ ) advection of energy, and 4) lateral ( $z$ ) advection of energy. The terms, $\alpha, \rho, h$, and $u$, represent the volume fraction, density, enthalpy, and velocity, respectively. The subscript, $k$, indicates the field; liquid, vapor, or droplet. The right-hand side terms include: 1) turbulent mixing of energy (lateral direction only), 2) the implicit heat transfer, 3) energy entering the 


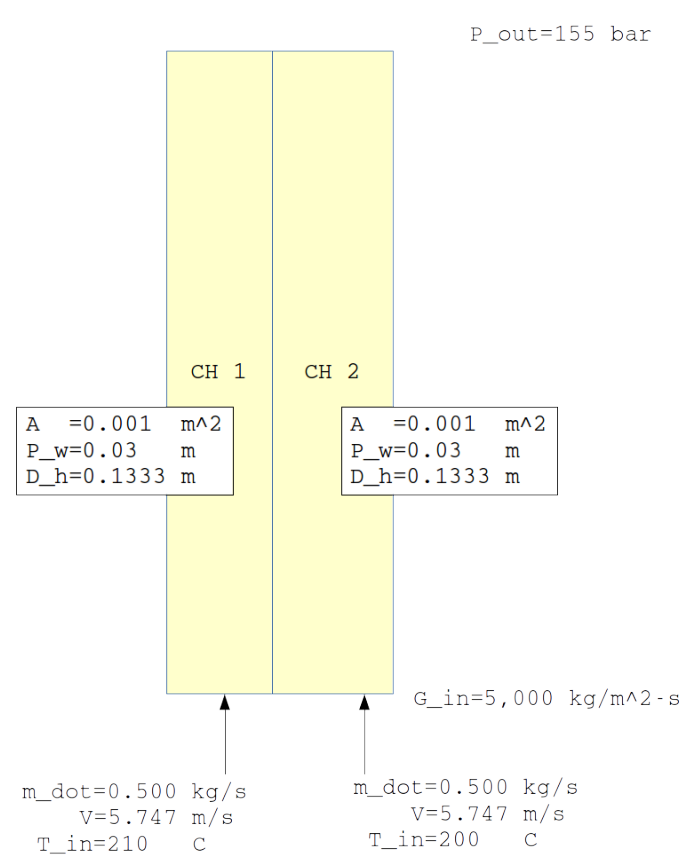

Figure 6.25: Model of problem for testing single-phase turbulent mixing of enthalpy. 
volume from the wall, and 4) the pressure-work on the volume over time. The term, $\Gamma^{\prime \prime \prime}$ represents the volumetric evaporation rate (transfer of mass from the liquid phase to the vapor phase).

We can make the following assumptions about this case:

1. The case is steady-state, eliminating the transient change in energy and the pressure work term.

2. The case is single-phase, eliminating all $k$ phase subscripts, void fractions, and the mass transfer term.

3. The case is unheated.

4. Because the case is set up so there is no lateral directed cross-flow, only the axial convective term remains.

The simplified equation, with $x$ being the axial direction, becomes

$$
\frac{\partial}{\partial x}(\rho h u)=q_{y}^{T^{\prime \prime \prime}}
$$

The density and velocity can be removed from the derivative because they are constants in the solution. This is applied directly to our problem of interest by formulating it for each channel in the model. Substituting the lateral transport of enthalpy due to turbulent mixing $\left(q_{y}^{T}\right)$ with the CTF form of the model yields the following set of equations:

$$
\begin{aligned}
& \dot{m}_{1} \frac{d}{d x} h_{1}+W_{1 \rightarrow 2}^{\prime}\left(h_{1}-h_{2}\right)=0 \\
& \dot{m}_{2} \frac{d}{d x} h_{2}-W_{1 \rightarrow 2}^{\prime}\left(h_{1}-h_{2}\right)=0
\end{aligned}
$$

Each equation is multiplied by the cross-sectional area to convert the velocity to mass flow rate and the volumetric mixing rate to a linear one. The $W_{1 \rightarrow 2}^{\prime}$ term represents the mixing rate of energy from Channel 1 to Channel 2. It has units of $\mathrm{kg} \mathrm{m}^{-1} \mathrm{~s}^{-1}$ and is defined as

$$
W_{1 \rightarrow 2}^{\prime}=\beta S_{12} \bar{G}
$$

The $\beta$ term is the turbulent mixing coefficient; it is the "tuning parameter" for the mixing model. Physically, it is a non-dimensional coefficient that represents the ratio of the lateral mass flux due to mixing to the axial mass flux. The other terms, $S_{12}$ and $\bar{G}$, are the gap width between Channels 1 and 2 (in $\mathrm{m}$ ) and the area-weighted average mass flux between the two channels (in $\left.\mathrm{kg} \mathrm{m}^{-2} \mathrm{~s}^{-1}\right)$. For this case, we set $\beta$ to a "typical" value of 0.0035 , the gap thickness based on problem geometry $(0.003 \mathrm{~m})$, and the mass flux based on problem operating conditions.

Returning to Equations 6.11 and 6.12 , we can solve the enthalpy distribution in a channel by relating the enthalpy in the two channels at any axial level, as follows: 


$$
\dot{m}_{1} h_{1}+\dot{m}_{2} h_{2}=\dot{m}_{1} h_{1, \text { in }}+\dot{m}_{2} h_{2, \text { in }}
$$

Because the mass flow rates in the channels are identical, this term cancels out and we are left with a relationship between the enthalpy in Channels 1 and 2 and the known inlet enthalpy boundary conditions. This is substituted into Equation 6.11 to develop a first-order, linear, ordinary differential equation that describes the enthalpy profile in Channel 2:

$$
\frac{d}{d x} h_{2}+\frac{2 W_{1 \rightarrow 2}^{\prime}}{\dot{m}} h_{2}-\frac{W_{1 \rightarrow 2}^{\prime}}{\dot{m}}\left(h_{1, \text { in }}+h_{2, \text { in }}\right)=0
$$

The solution of this equation is

$$
h_{2}=\frac{1}{2}\left(h_{1, \text { in }}+h_{2, \text { in }}\right)+C \exp \left(\frac{-2 W_{1 \rightarrow 2}^{\prime}}{\dot{m}} x\right) .
$$

Using the inlet enthalpy boundary conditions, the value of the constant, $C$ is determined. The same process is repeated for the first channel, leading to the following final solutions for enthalpy distribution in the system due to turbulent mixing.

$$
\begin{aligned}
& h_{1}=\frac{1}{2}\left(h_{1, \text { in }}+h_{2, \text { in }}\right)-\frac{1}{2}\left(h_{2, \text { in }}-h_{1, \text { in }}\right) \exp \left(\frac{-2 W_{1 \rightarrow 2}^{\prime}}{\dot{m}} x\right) \\
& h_{2}=\frac{1}{2}\left(h_{1, \text { in }}+h_{2, \text { in }}\right)+\frac{1}{2}\left(h_{2, \text { in }}-h_{1, \text { in }}\right) \exp \left(\frac{-2 W_{1 \rightarrow 2}^{\prime}}{\dot{m}} x\right)
\end{aligned}
$$

CTF Model Description The CTF input deck is set up from Figure 6.25. Axial meshing is set to $2.54 \mathrm{~cm}$. The gap thickness is set to $0.003 \mathrm{~m}$ and its length is set to $0.0126 \mathrm{~m}$, which would result from a PWR lattice with $12.6 \mathrm{~mm}$ pitch and $9.5 \mathrm{~mm}$ rod outside diameter. The turbulent mixing model is set so that a single-phase turbulent-mixing parameter, $\beta$, could be set equal to the value used in the analytical solution. The liquid density is set to a constant value of $700 \mathrm{~kg} \mathrm{~m}^{-3}$. Note, however, that density does not appear in the analytical solution and, thus, has no impact on the CTF results in terms of turbulent mixing of enthalpy.

Discussion of Results The case is run to steady-state in CTF and the axial enthalpy profile is extracted for each channel. Figure 6.26 shows results for the two channels compared with the analytical solution that is calculated above. The figure demonstrates excellent agreement between code and expected results.

As noted above, the turbulent mixing model is defined so that it predicts net transfer of the parameter of interest due to turbulent exchange between channels. This transfer may or may not include mass transfer. For a single-phase, unheated case, turbulent exchange leads to zero net mass transfer because each unit of liquid volume that moves from Channel 1 to 2 is replaced with an equal volume that moves from Channel 2 to 1 . The exchange does cause enthalpy to be 


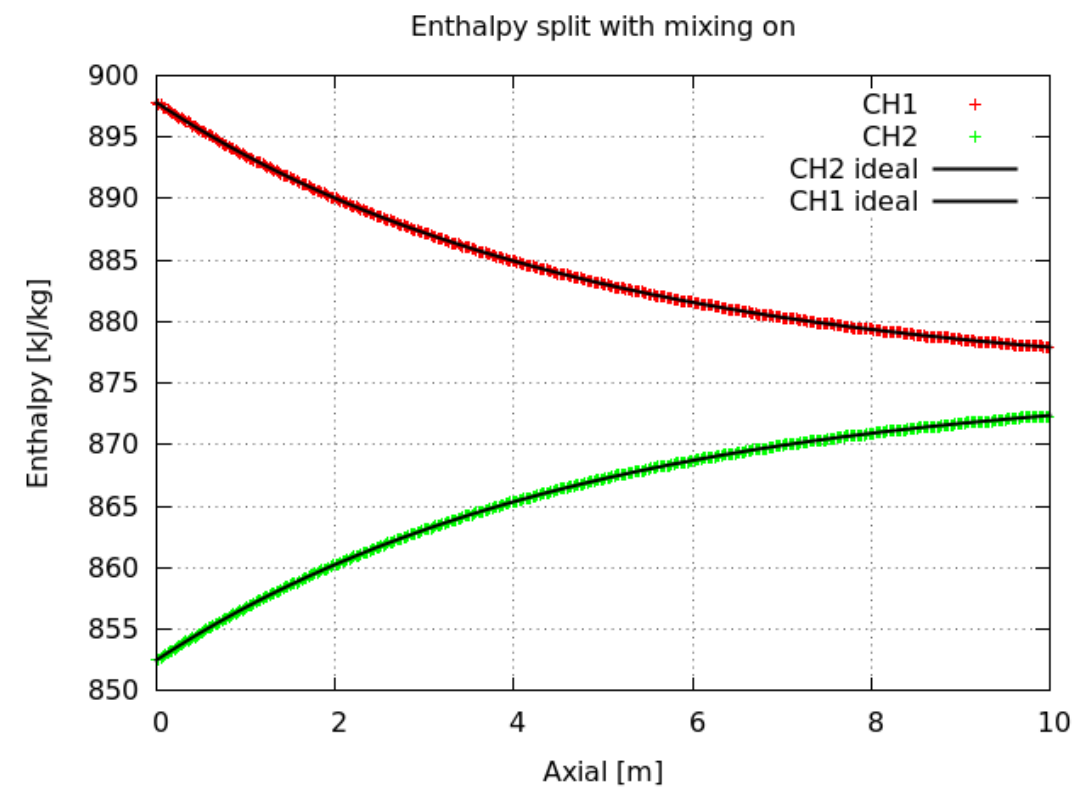

Figure 6.26: Comparison of CTF-predicted liquid enthalpy in channels and the analytical solution.

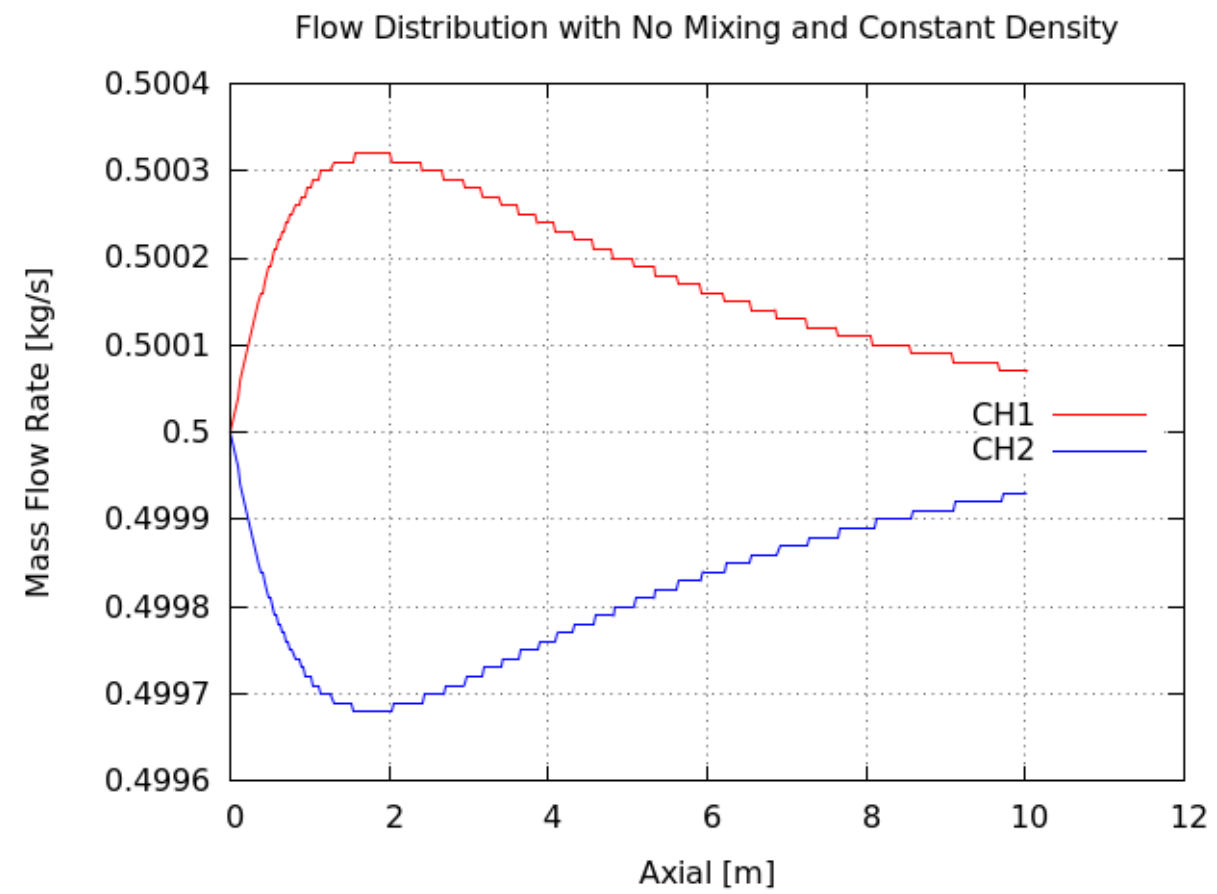

Figure 6.27: CTF-predicted axial mass flow rate profile in the channels. 


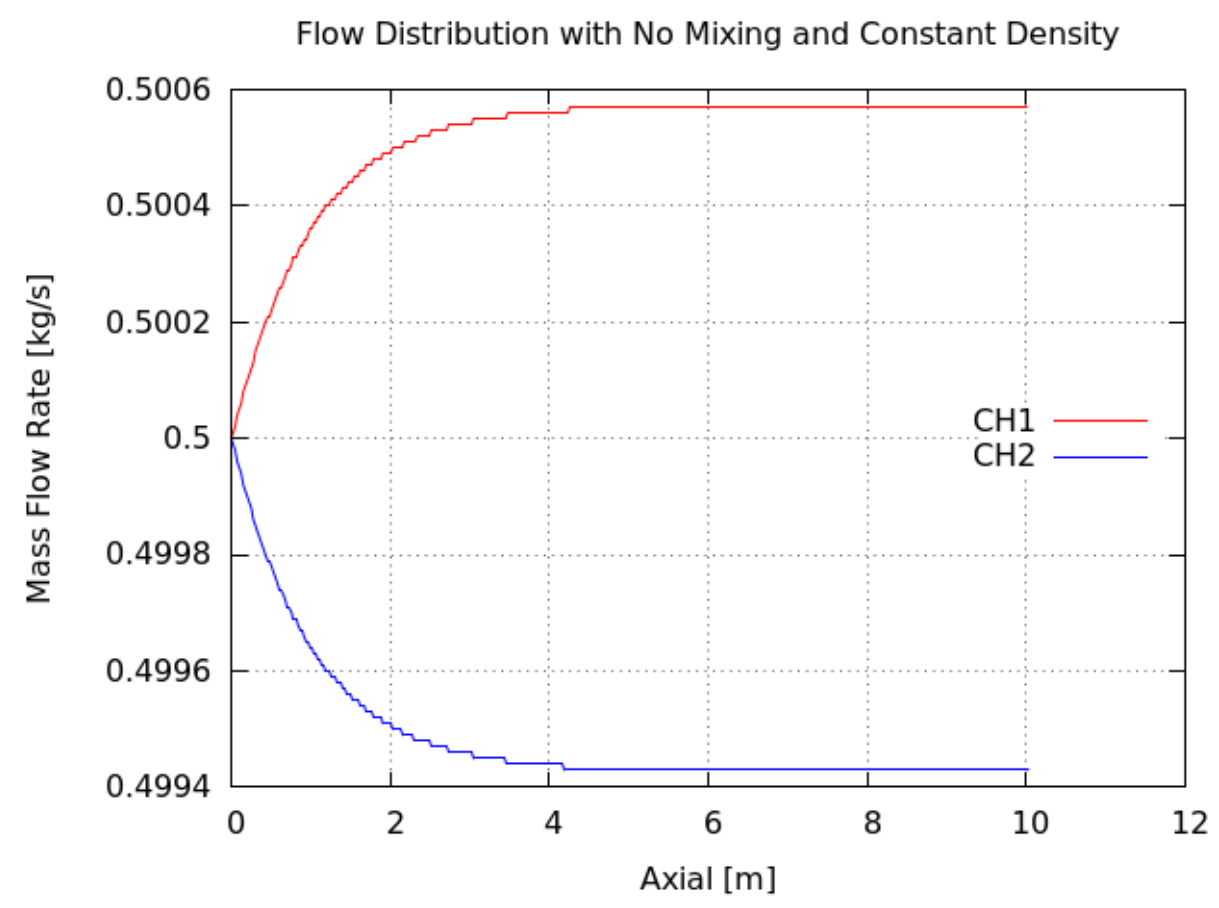

Figure 6.28: CTF-predicted axial mass flow rate profile in the channels with turbulent-mixing disabled.

moved, as is observed in Figure 6.26. A check is made on the axial mass flow rate profile in each of the channels in Figure 6.27.

It is noted that there is a slight discrepancy in the axial mass flow rate profile. Ideally, the normalized mass flux should be zero everywhere; however, the plot indicates that it varies by as much as $0.13 \%$ from the average mass flux. This discrepancy is further investigated by disabling the turbulent-mixing and void drift, leading to the flow redistribution shown in Figure 6.28.

The magnitude of the flow redistribution is small; however, results indicate that something in the solution is causing a slight drift in mass flux despite the channels being geometrically identical. 


\section{CHAPTER 7}

\section{VOID CONTENT}

\section{$7.1 \quad$ PSBT}

Void measurements were taken at three axial locations in the rod-bundle facility. The measurements represent the average void of the four subchannels surrounding the central rod. Table 2.4.1 of the specification[3] states that the rod-bundle void measurement uncertainty is $4 \%$. To demonstrate CTF's capability to predict the void content in the flow, the predicted void (y-axis) is compared to measured void (x-axis) for the three test series. Results are shown in Figures 7.1, 7.2, and 7.3. In these figures, data that lands on the solid red line is in perfect agreement with the experimental results. The dashed green lines depict the experimental $2 \sigma$ uncertainty of $4 \%$. Data that falls within these dashed green boundaries are in agreement with experimental results to within experimental uncertainty.

The RMSE is provided for the three experimental series in Table 7.1. The statistics are provided for each test and each measurement location. Also, a summary of the statistics for all data in the series are given in the last row of the table.

Looking at the results of Table 7.1, there is no noticeable trend in error with respect to either test series or measurement location. In fact, the total test errors for each series are all pretty similar. We find that the RMSE comes out to about 0.053 for the entire group of tests, which sits just outside the experimental uncertainty of $4 \%$. Considering that the RMSE calculation will magnify the larger errors, this is a very close agreement with experimental results.

The PSBT tests will exercise the inter-phase-mass-transfer (evaporation/condensation) and boiling models in CTF. Because the CTF predicted void around the center rod is being compared to measurements taken at the same location (as opposed to a bundle-average void), these tests will also exercise the void-drift model in CTF. 


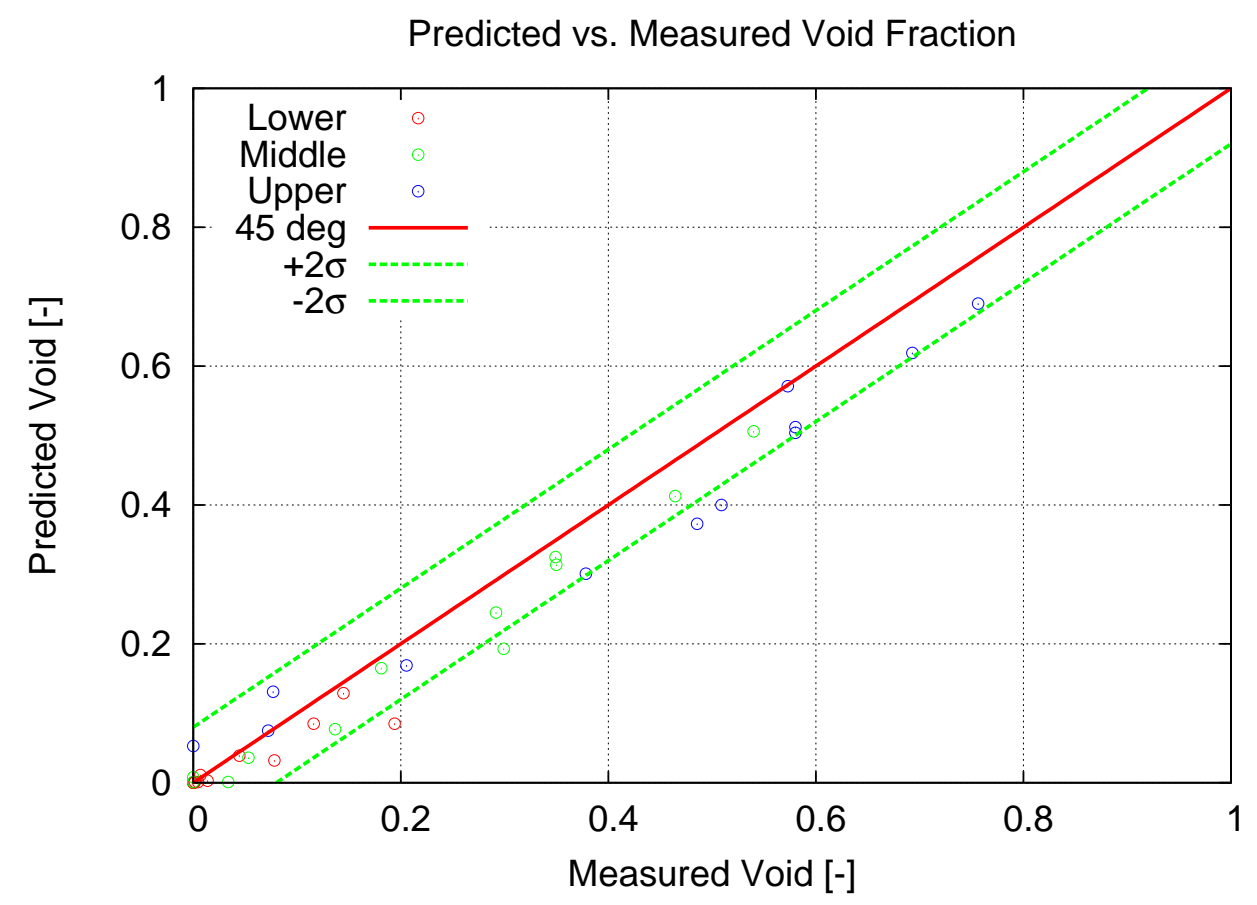

Figure 7.1: PSBT Series 5 predicted versus measured void around central rod in bundle

Table 7.1: RMSE of measured and predicted void for PSBT Series 5, 6, and 7

\begin{tabular}{|l|c|c|c|}
\hline Location & Series 5 & Series 6 & Series 7 \\
\hline lower & 0.036 & 0.065 & 0.032 \\
mid & 0.045 & 0.046 & 0.072 \\
upper & 0.069 & 0.057 & 0.041 \\
\hline total & 0.052 & 0.056 & 0.051 \\
\hline
\end{tabular}




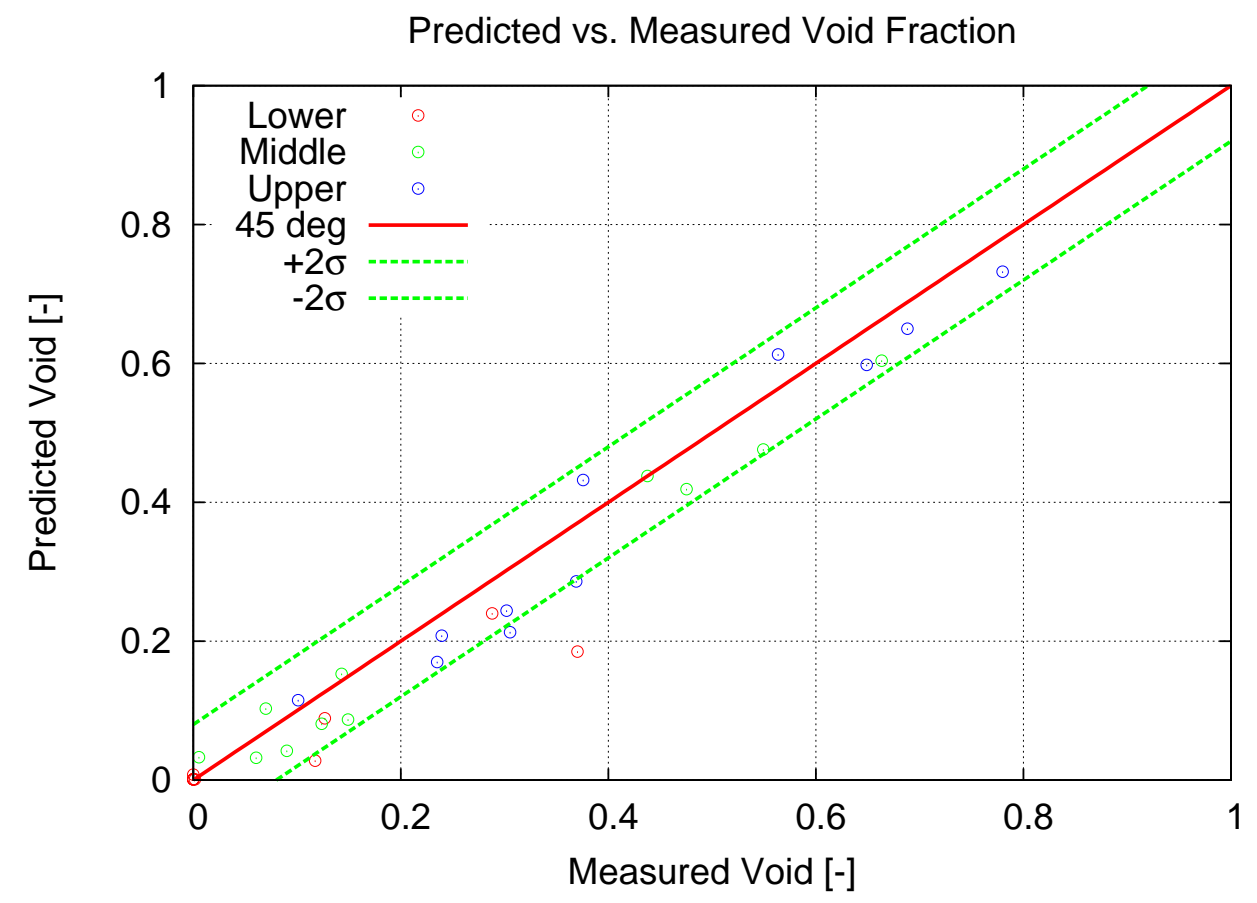

Figure 7.2: PSBT Series 6 predicted versus measured void around central rod in bundle 


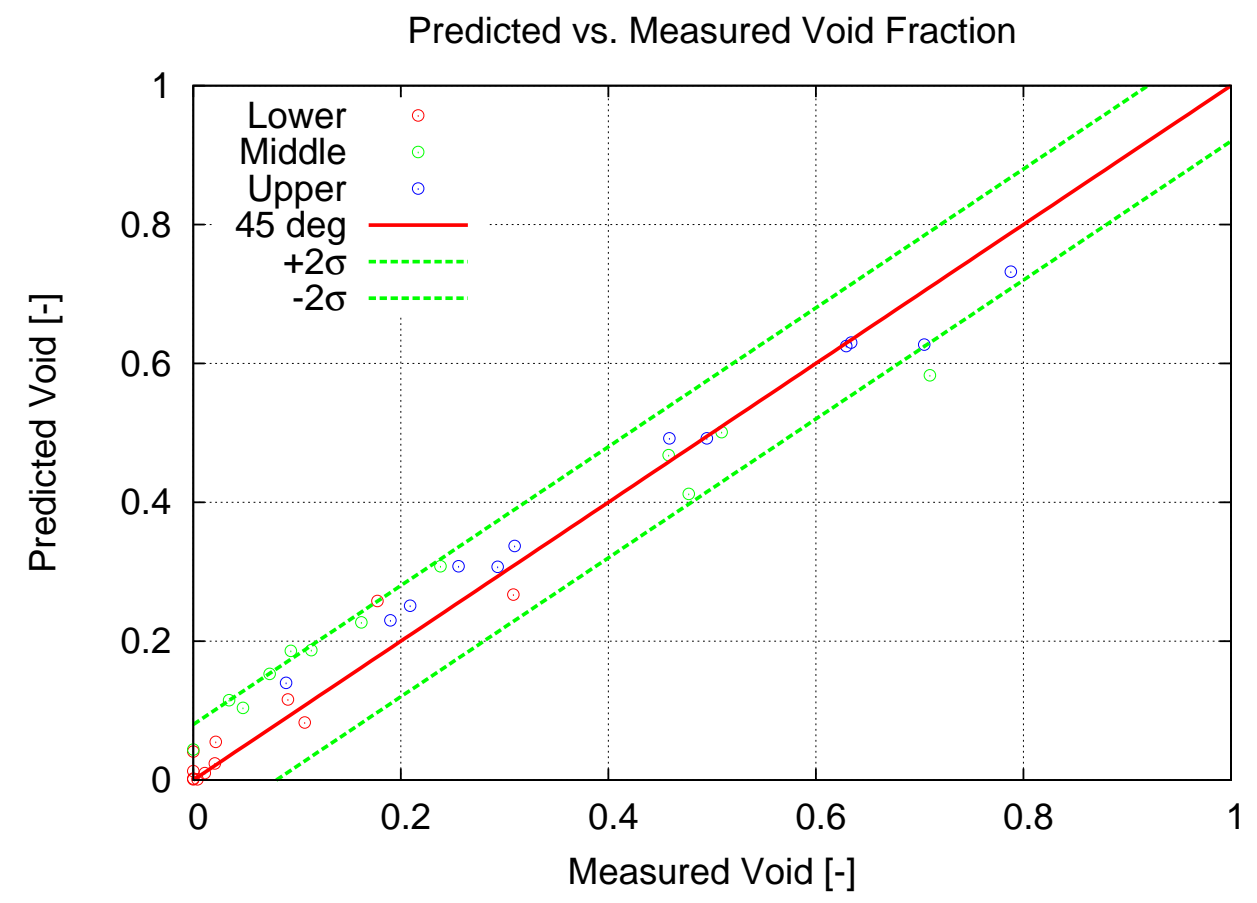

Figure 7.3: PSBT Series 7 predicted versus measured void around central rod in bundle 


\subsection{FRIGG Facility}

The CTF prediction of the bundle-averaged void profile in the FRIGG facility is shown in Figure 7.4. The RMSE was calculated from all of the points that were sampled from the experimental results (summarized in Table 7.2). The RMSE is 0.0336, showing close agreement with experimental results.

Table 7.2: Summary of void fractions sampled from FRIGG data report

\begin{tabular}{|c|c|}
\hline Axial Location $(\mathrm{m})$ & Measured Void $(-)$ \\
\hline 0.824 & 0.252 \\
1.291 & 0.304 \\
1.921 & 0.469 \\
2.388 & 0.618 \\
2.972 & 0.607 \\
3.567 & 0.676 \\
4.034 & 0.696 \\
\hline
\end{tabular}




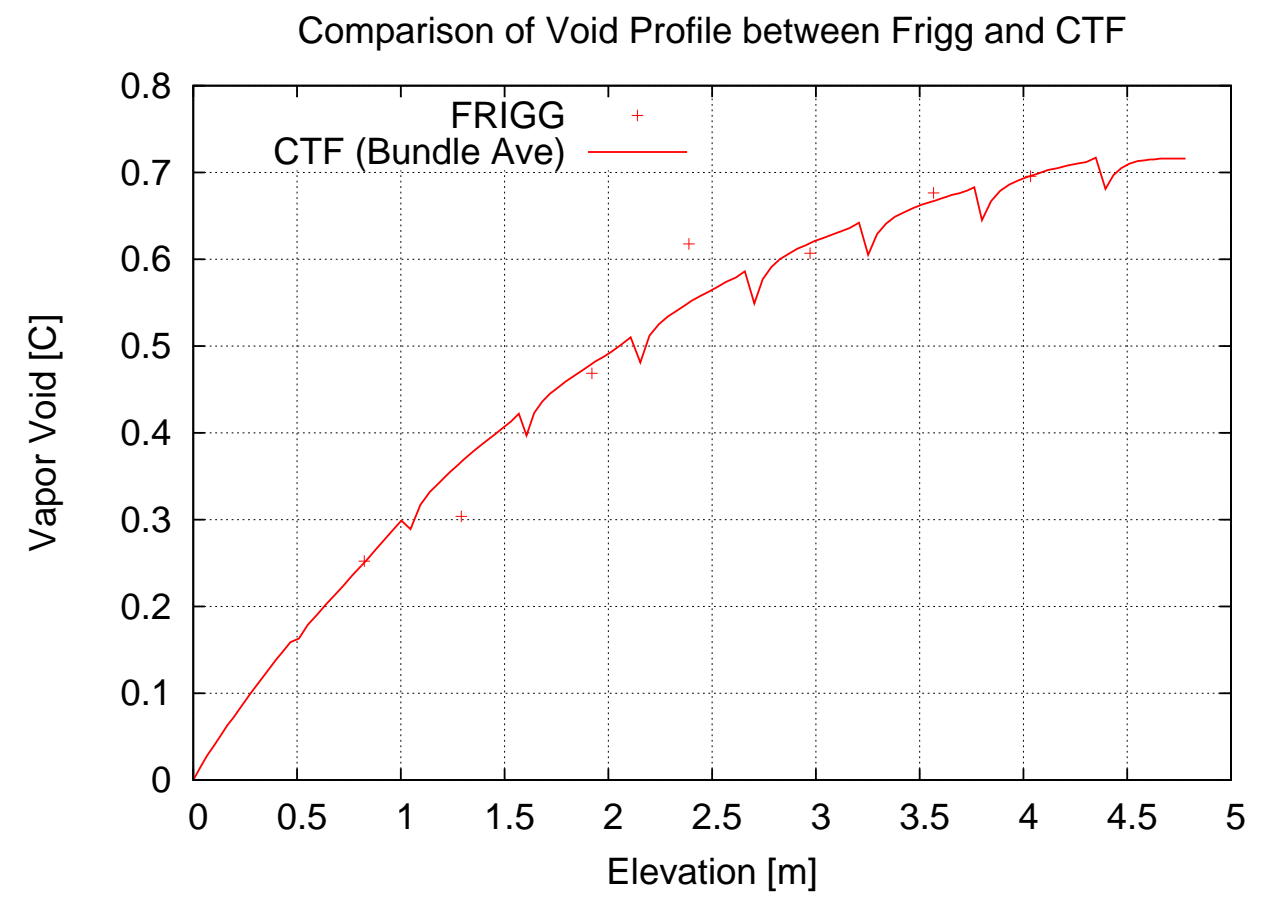

Figure 7.4: Predicted and measured axial bundle-averaged void profile in FRIGG facility 


\section{CHAPTER 8}

\section{TWO-PHASE TURBULENT MIXING AND VOID DRIFT}

\subsection{Validation}

\subsubsection{GE $3 \times 3$}

A comparison of the measured and predicted exit equilibrium qualities is provided in Figure 8.1 for the base case model, which uses the void drift model with $K_{a}$ set to 1.4, the turbulent mixing model with $\beta_{\mathrm{sp}}$ set to 0.007 , and $\Theta_{M}$ set to 5.0. Figure 8.1 shows that most predicted exit qualities fall within experimental uncertainty. However, the values that seem to vary furthest from measured results are qualities in the corner type subchannel. Root-mean-square of the error (RMSE) (Equation 1.3) values for the three subchannel types are shown directly in the figure.

We see that, on average, the corner channel quality prediction error is about double the inner and side type channel prediction error. Furthermore, quality in the corner channels is typically over-predicted by CTF. The side and inner type exit qualities, however, generally match the experimental values within the measurement uncertainty.

If the void drift model is disabled, comparisons with experimental data become much worse. Figure 8.2 shows the exit equilibrium quality compared with experimental measurements with the void drift model disabled. Corner channel RMSE increases from 0.044 to 0.121 and inner channel RMSE increases from 0.012 to 0.019 for exit quality.

In addition to the exit equilibrium quality, the authors also measured the exit mass flux of each individual subchannel. Results for the two-phase experiments are shown in Figure 8.3. The rRMS for the two-phase experiments are larger than for the single-phase results shown in Figure 6.23. The corner is still the most poorly predicted of all channel types. Figure 8.4 shows the exit mass flux for the two-phase cases when void drift is disabled. The accuracy of both corner and inner channel type predictions degrades sharply. Similarly, mass flux rRMS more than doubles for the corner channel type when the void drift model is disabled. 
The original GE $3 \times 3$ study used the Rogers \& Rosehart correlation to set the single-phase mixing coefficient. The results are repeated here for all experiments in Figures 8.5 and 8.6 for mass flux and quality. Prediction of both measured quantities improves when using Rogers \& Rosehart over the constant $\beta$ value of 0.007 . Results indicate there is almost no difference in accuracy of the results between using the Rogers \& Rosehart correlation to calculate single-phase mixing coefficient or the constant value of 0.007 .

The axial mass flux distribution of each channel type is also investigated. It is discovered that the distributions are complicated, so the spacer grids are removed for the sake of clarifying two-phase and flow-regime dependant trends. The turbulent-mixing and void drift models are enabled, with the single-phase mixing coefficient being set to 0.007 with the Beus two-phasemixing multiplier set to 5.0. The following plots show the normalized mass flux in each channel type, calculated as local mass flux minus bundle average mass flux and divided by bundle average mass flux. The measured normalized mass fluxes are shown with horizontal dashed lines. The measured value is only available at the outlet. Different colored lines are used to denote different channel types; red for corner, blue for side, and green for inner.

The plots also include the flow regime, denoted with colored regions in the plot; SP stands for single-phase, SB for small bubble, SL for slug, CH for churn, and AN for annular. The flow regimes often change at different axial locations in different channel types. The gray shaded regions show the length of this transition region. For example, the green "SB" region means that all channel types are in the small bubble flow regime. When the gray region begins, it means at least one of the channel types has transitioned to slug flow, but at least one channel type is still in small bubble. When the gray region ends, it means that all channels are in slug. The flow quality is shown for each channel type using dot-dashed lines, which corresponds to a secondary $y$-axis.

The high-flow, low-power cases, $2 \mathrm{C} 1$ and 2C2, are shown in Figures 8.7 and 8.8. The figures show that the corner mass flux tends to decrease and inner mass flux tends to increase until the flow quality starts to increase. At this point, the void drift effect begins to draw the normalized mass fluxes back toward zero. Test $2 \mathrm{C} 2$ has lower subcooling, so this effect starts sooner in the bundle. In Case 2C2, there is a "notch" in the axial mass flux profile for the corner channel. It is found that this is due to entrainment starting by running a case with the droplet field disabled.

The axial flow behavior is fairly smooth in the "C"-series cases compared with higher power cases and lower flow cases. Test $2 \mathrm{G} 1$ has the power doubled compared with the $2 \mathrm{C} 1$ and $2 \mathrm{C} 2$ cases. Its flow distribution is shown in Figure 8.9. Because of high subcooling, the exit flow quality is still only about $10 \%$, but the flow distributions look more chaotic starting at about the $0.8 \mathrm{~m}$ mark. The flow distribution appears to begin changing in the slug flow regime and transition to churn.

The "D"-series tests have the highest power-to-flow ratio. Test 2D3 makes it into the annular flow regime as a result. Its axial flow distribution is shown in Figure 8.10. Flow quality goes as high as almost $40 \%$. The flow distribution experiences a significant trend reversal at about $1.5 \mathrm{~m}$, as the channels transition into the annular flow regime. Again, there is a "notch" in the corner channel mass flux distribution due to the start of entrainment.

The overall trend seems for flow to migrate from the corner channel to the side and inner channels when flow is low void. At the start of bulk boiling, this trend reverses, causing channel flow to stay the same or move back to the average. The transition to the annular flow regime 


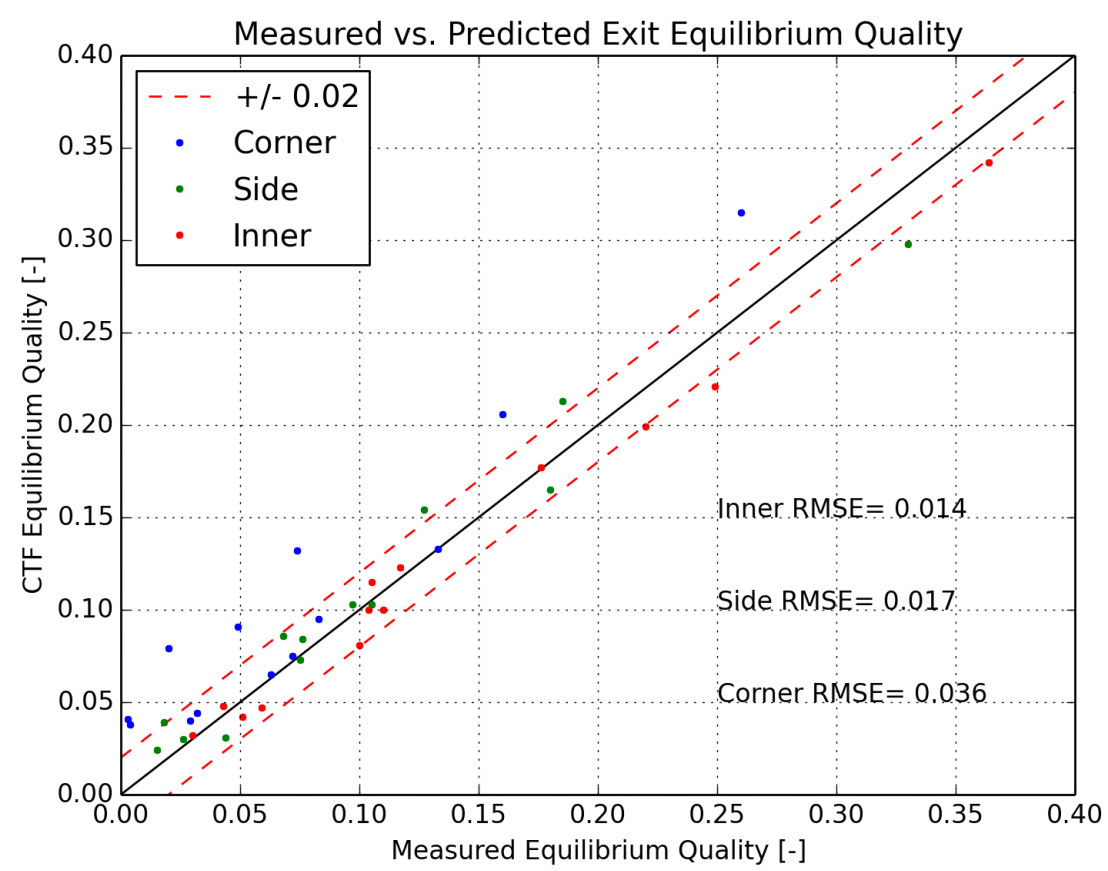

Figure 8.1: Comparison of predicted and measured exit equilibrium quality for GE $3 \times 3$ two-phase cases.

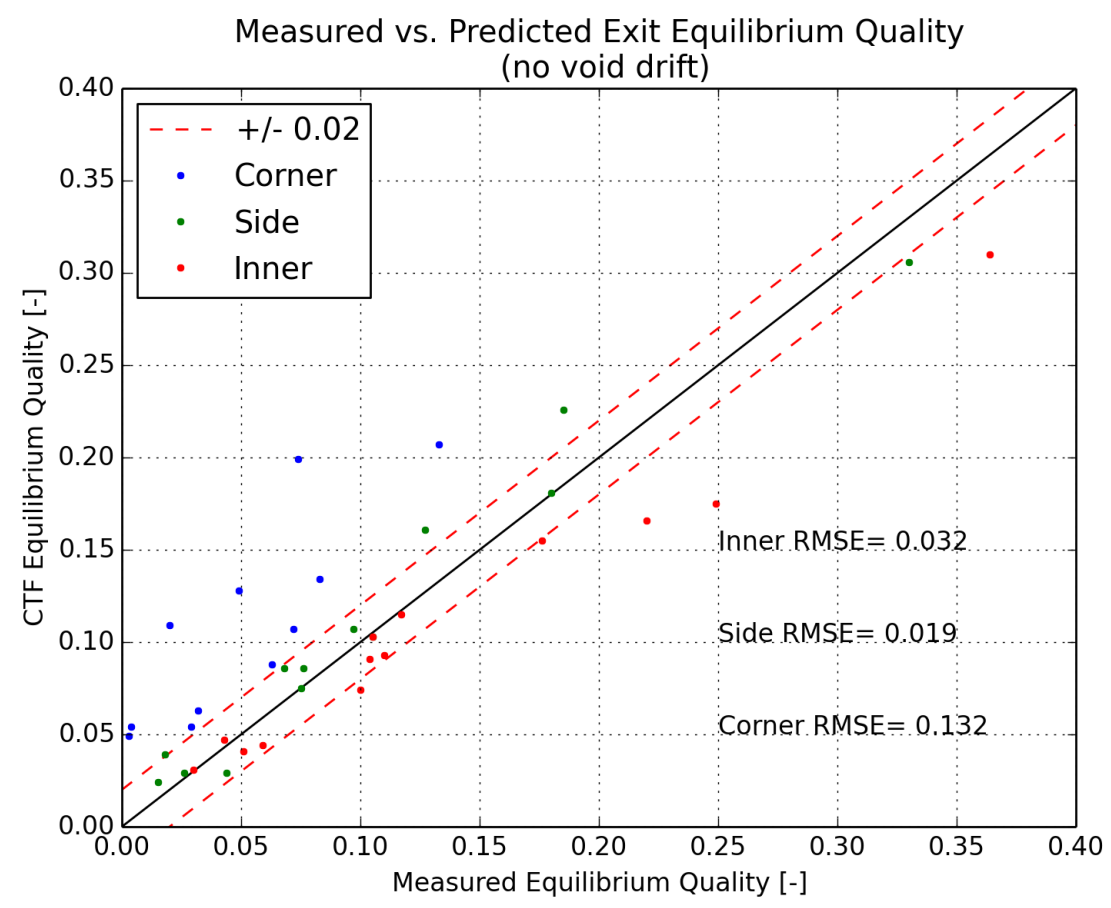

Figure 8.2: Comparison of predicted and measured exit equilibrium quality for GE $3 \times 3$ two-phase cases when void drift is disabled. 


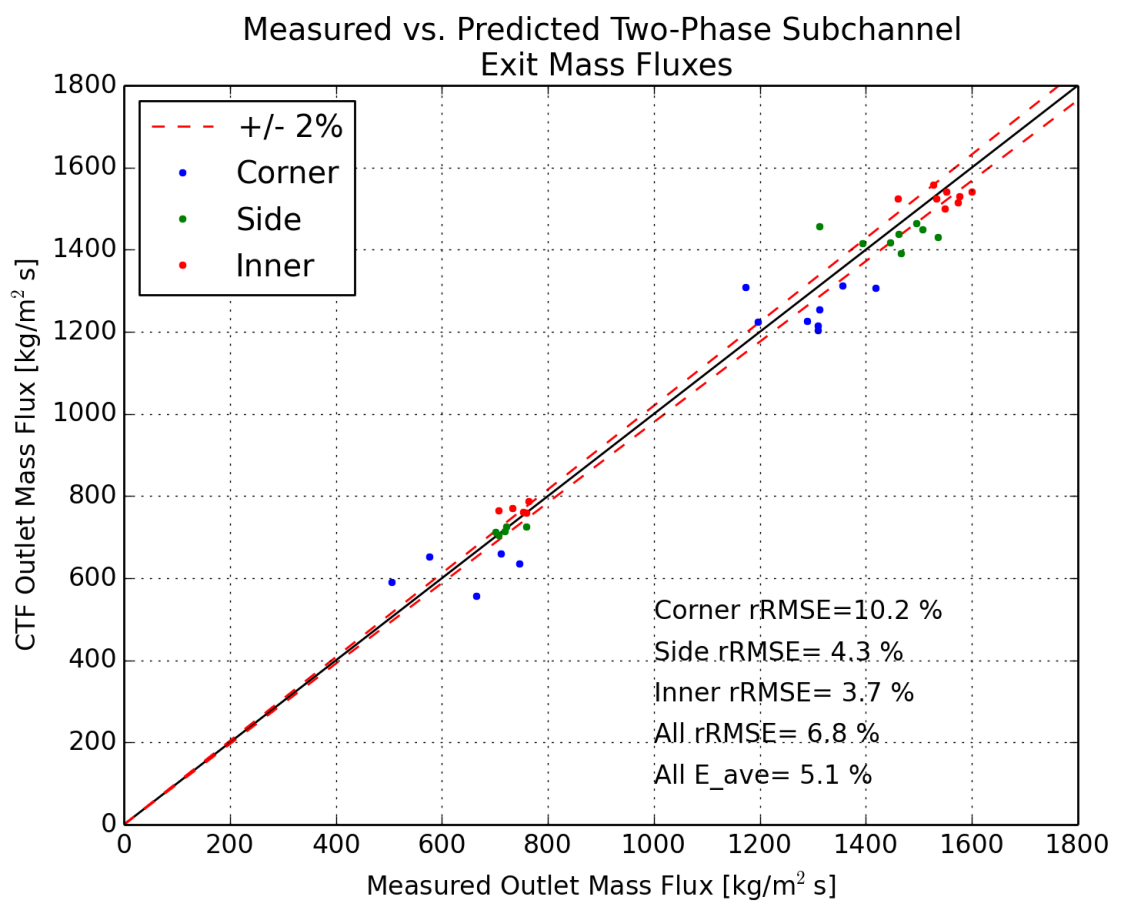

Figure 8.3: Comparison of predicted and measured exit mass flux for GE $3 \times 3$ two-phase cases.

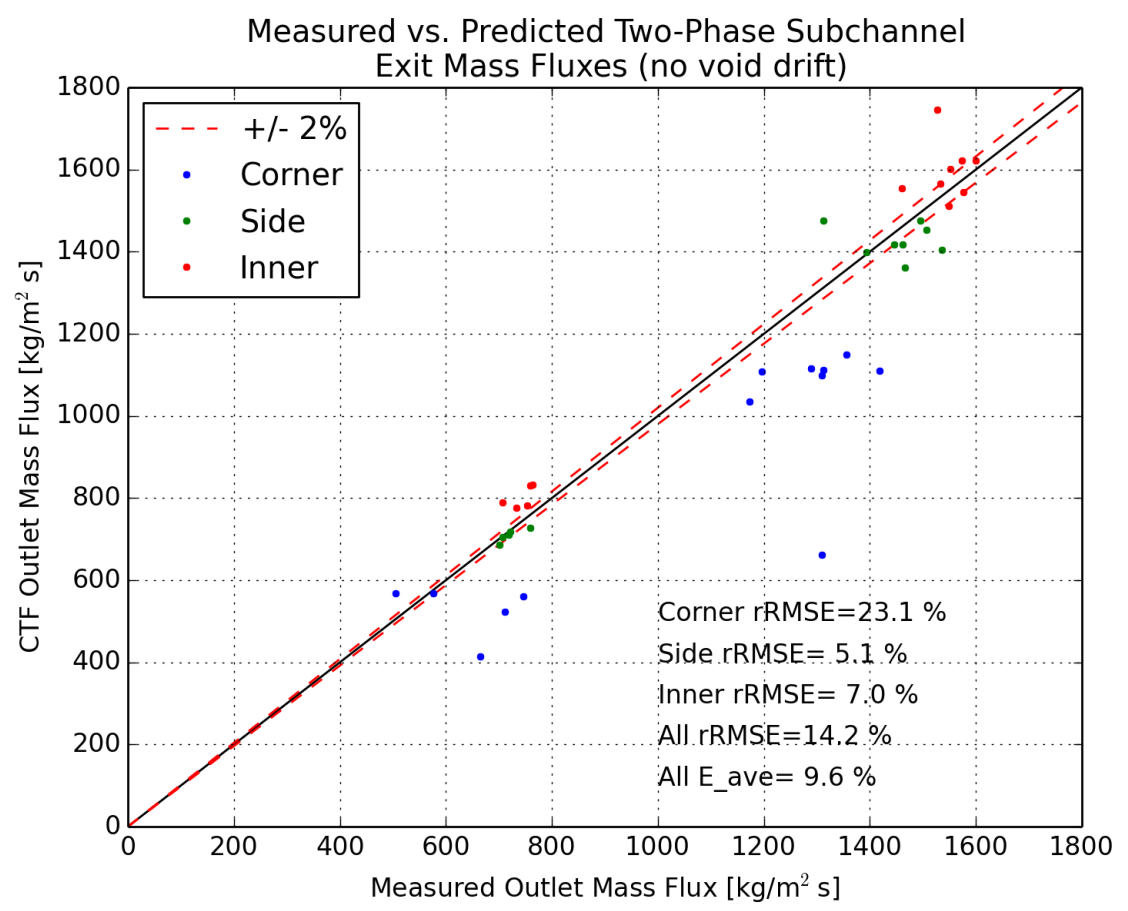

Figure 8.4: Comparison of predicted and measured exit mass flux for GE $3 \times 3$ two-phase cases when void drift is disabled. 


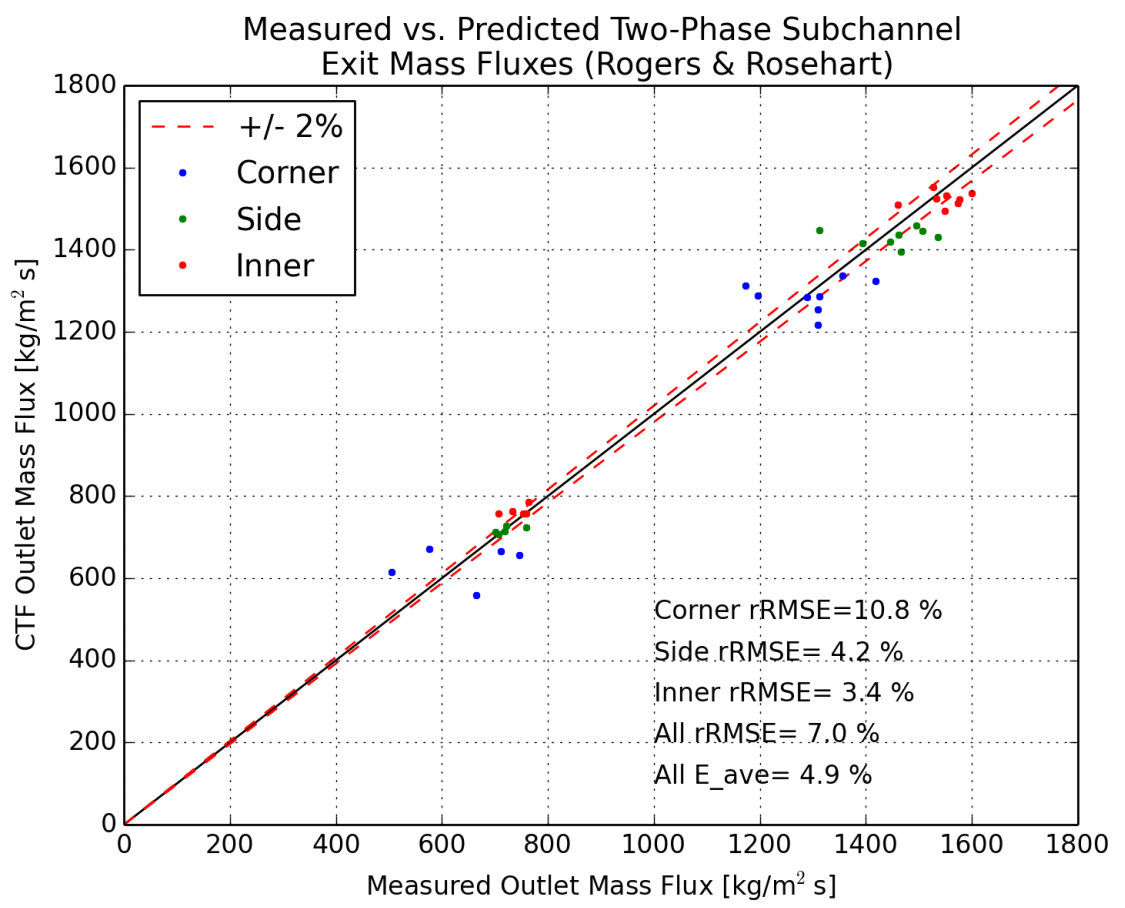

Figure 8.5: Comparison of predicted and measured exit mass flux for GE $3 \times 3$ two-phase cases using Rogers \& Rosehart.

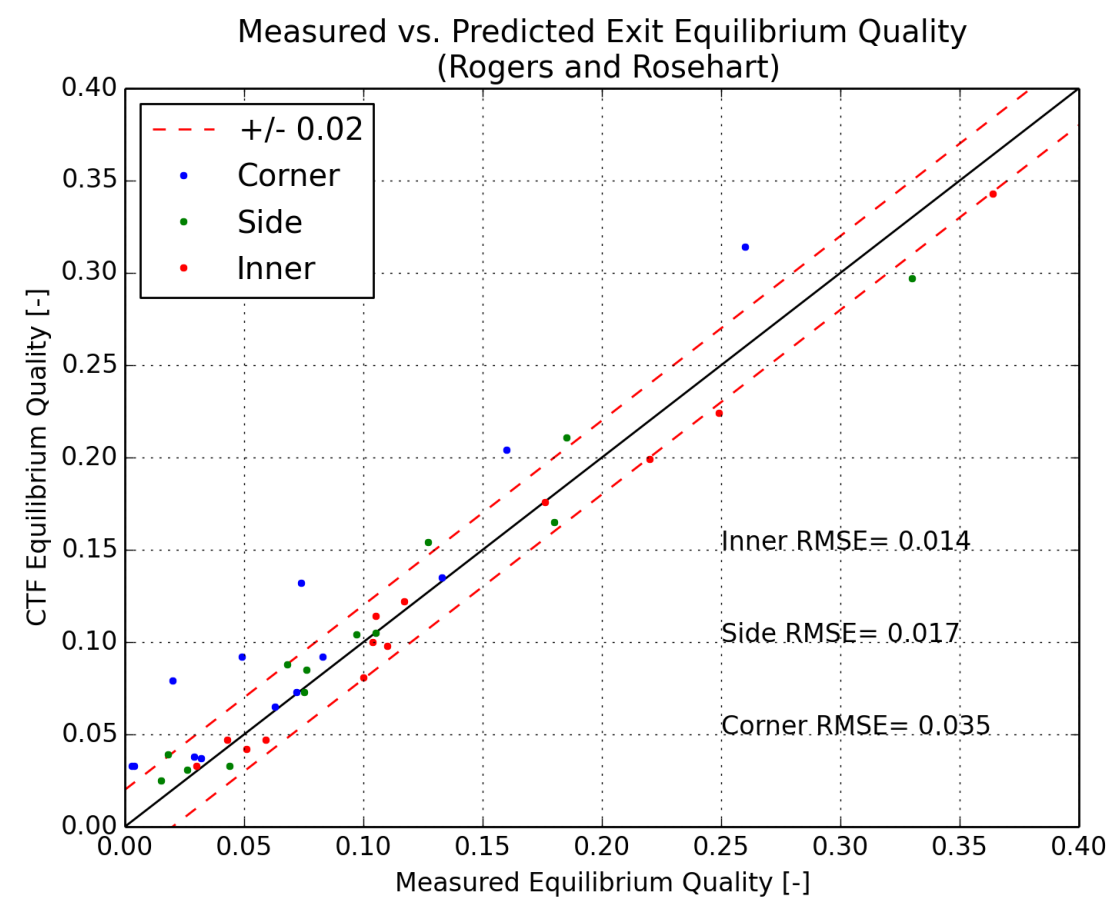

Figure 8.6: Comparison of predicted and measured exit quality for GE $3 \times 3$ two-phase cases using Rogers \& Rosehart. 


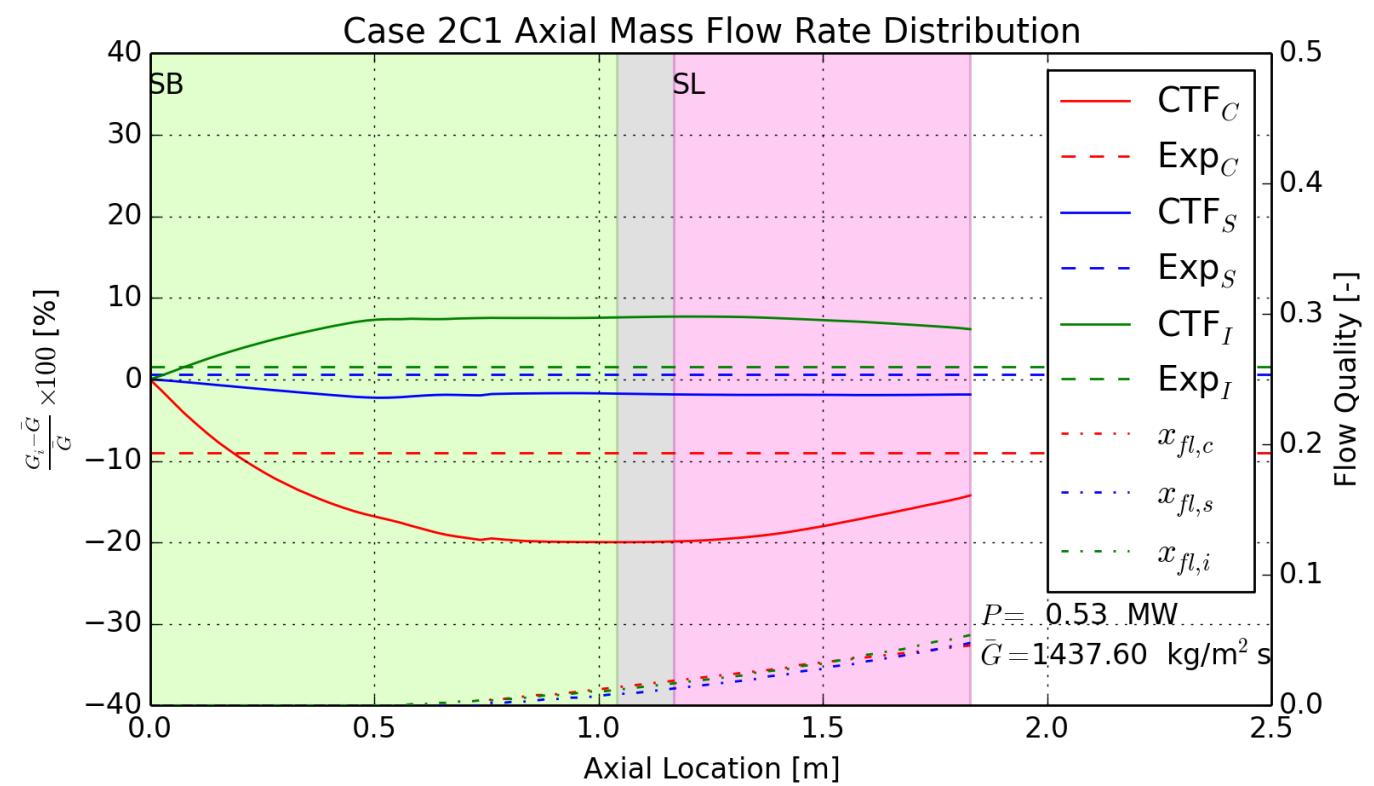

Figure 8.7: GE $3 \times 3$ Test 2 C1 CTF-predicted axial flow distribution and flow quality distribution compared with experimental measurement.

causes a second reversal, making flow migrate out of the corner channel and back into the inner and side channels due to a change in constitutive models. 


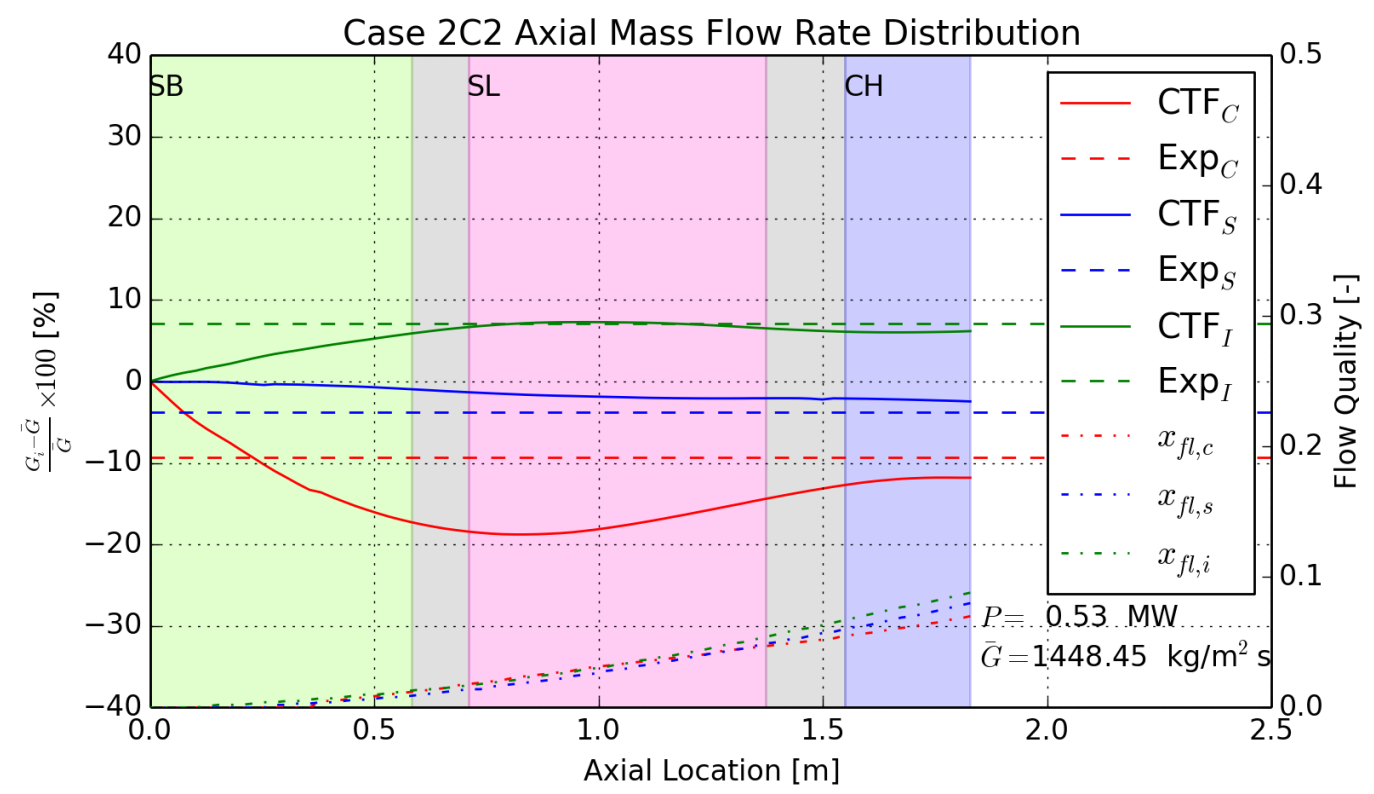

Figure 8.8: GE $3 \times 3$ Test 2 C2 CTF-predicted axial flow distribution and flow quality distribution compared with experimental measurement.

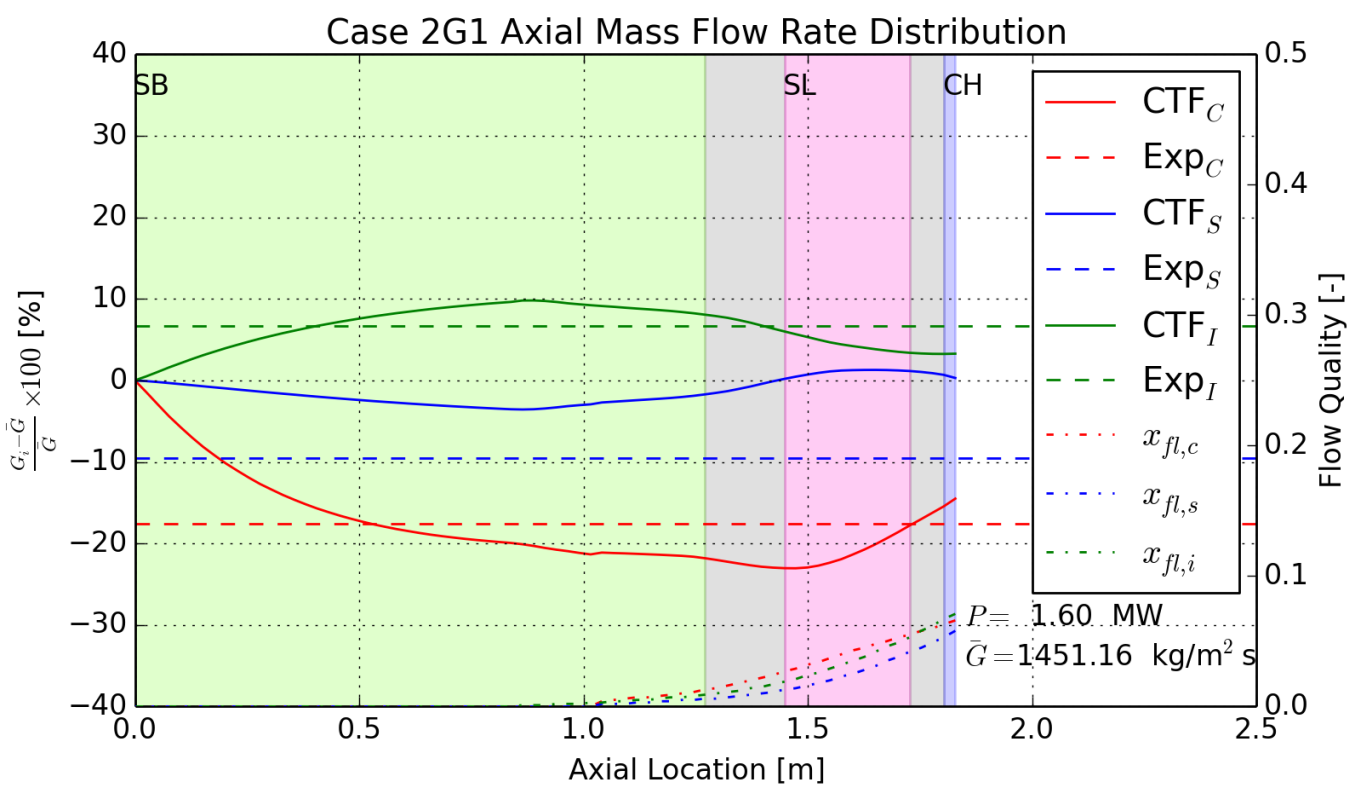

Figure 8.9: GE $3 \times 3$ Test 2G1 CTF-predicted axial flow distribution and flow quality distribution compared with experimental measurement. 


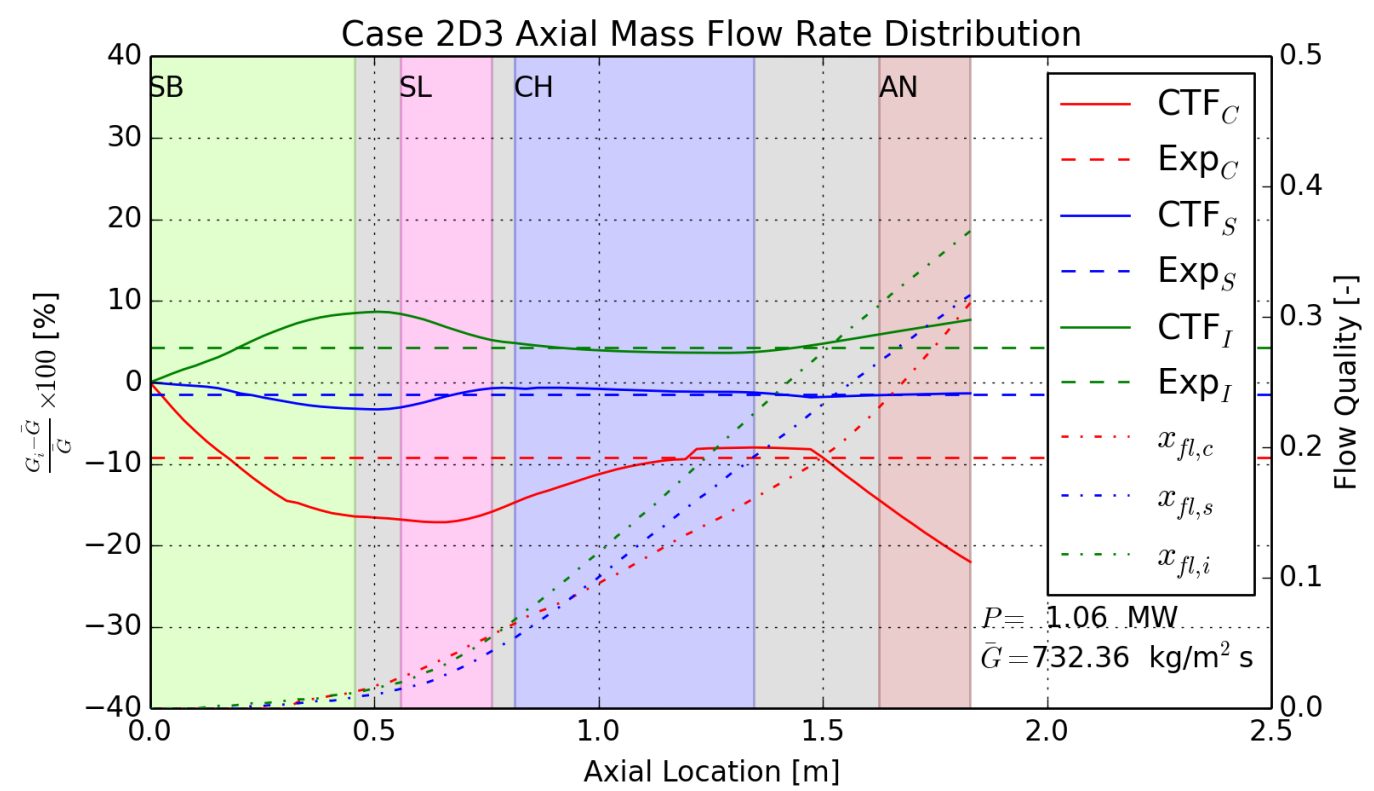

Figure 8.10: GE $3 \times 3$ Test 2D3 CTF-predicted axial flow distribution and flow quality distribution compared with experimental measurement.

\subsubsection{BFBT $8 \times 8$}

The process of running the simulations, extracting data from CTF output files, and creating plots is scripted for automated regeneration of analysis results. Most of the cases run by CTF converged to within tolerance. Convergence is checked by checking a collection of engineering parameters of interest:

1. Global energy balance (energy into the system minus energy out),

2. Energy movement between fields,

3. Global mass balance (mass into the system minus mass out),

4. Mass movement between fields due to evaporation/condensation,

5. Fluid energy storage between time steps,

6. Solid energy storage between time steps, and

7. Mass storage between time steps.

All of these terms need to drop below a user-set tolerance. The tolerance for these cases is initially set to the same tolerance used for PWR cases. All cases except for two-0011-61 and 1071-61 - are able to converge to steady-state within the PWR convergence criteria when the droplet field is disabled. When the droplet field is enabled, two additional cases fail to 


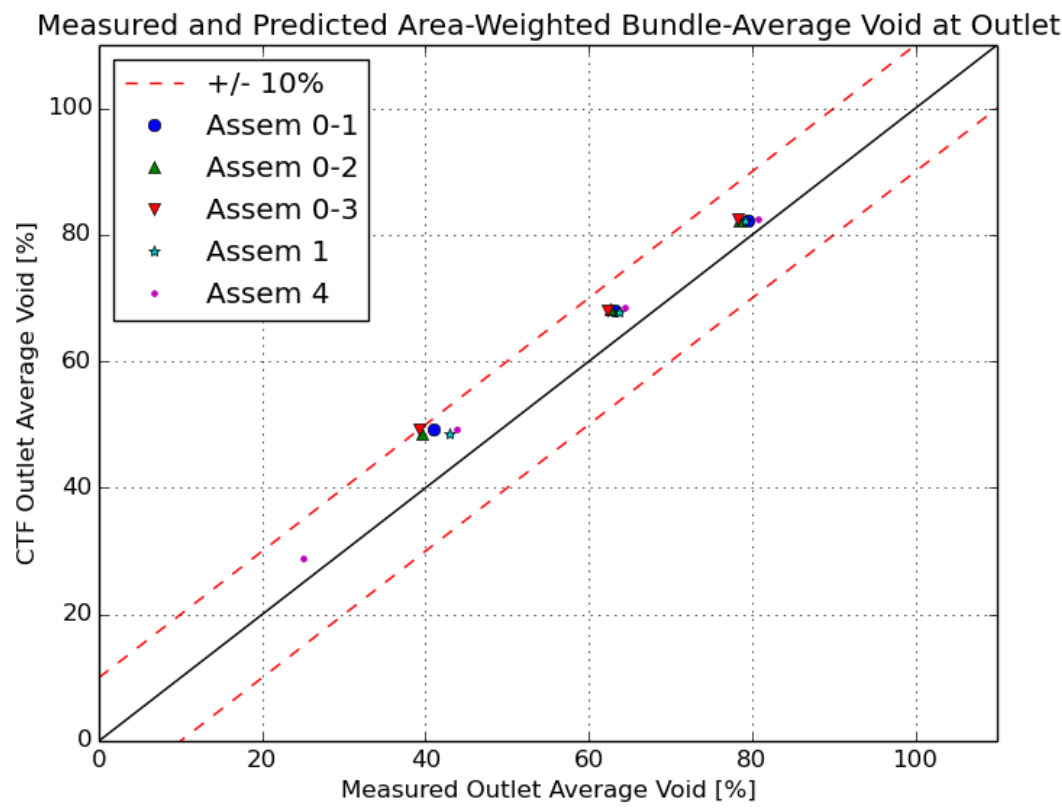

Figure 8.11: Comparison of measured and predicted bundle-averaged outlet void.

converge to within the PWR tolerance-1071-55 and 4101-61. It is expected that CTF will not be able to converge as tightly for high-void cases, where there is a great deal of mass and energy transfer happening between the phases, as it does for single-phase cases. The fact that the rod conduction equation solution is explicitly coupled to the fluid energy solution, rather than an implicit coupling, likely worsens this problem.

The tolerance of the non-converging cases has to be increased to $1 \%$ for the energy balances, the mass balances, and the mass storage (Items 1, 2, 3, 4, and 5 from the list above). The tolerance for fluid energy storage has to be increased to $2 \%$. The tolerances used for PWR cases are $0.01 \%$ for Items $1,2,3$, and 4 and $0.5 \%$ for Items 5,6 , and 7 . Item 6 , the solid energy storage, has no impact on these simulations because the solids (heater rods, water rods, and fuel canister) are not modeled in CTF for these cases.

As a first step, CTF-predicted bundle average void at the outlet and thermal equilibrium quality is compared with the experimental results. The thermal equilibrium quality is calculated from the flow-weighted mixture enthalpy of individual channels. The exit void is calcualted from the area-weighted void of individual channel values. he comparison of CTF-predicted and measured bundle average outlet void is shown in Figure 8.11 and the comparison of CTFpredicted and measured bundle average thermal equilibrium quality is shown in Figure 8.12.

There is a tendency for CTF to consistently overpredict the bundle-average void. The overprediction is more severe for the cases with lower amounts of void (about 40\%). At outlet void of about $80 \%$, the prediction almost matches the experimental values. If the same amount of energy is entering the CTF model as is entering the experimental facility, the mixture enthalpy at the outlet should be identical to the experiment (barring steam table differences); however, 


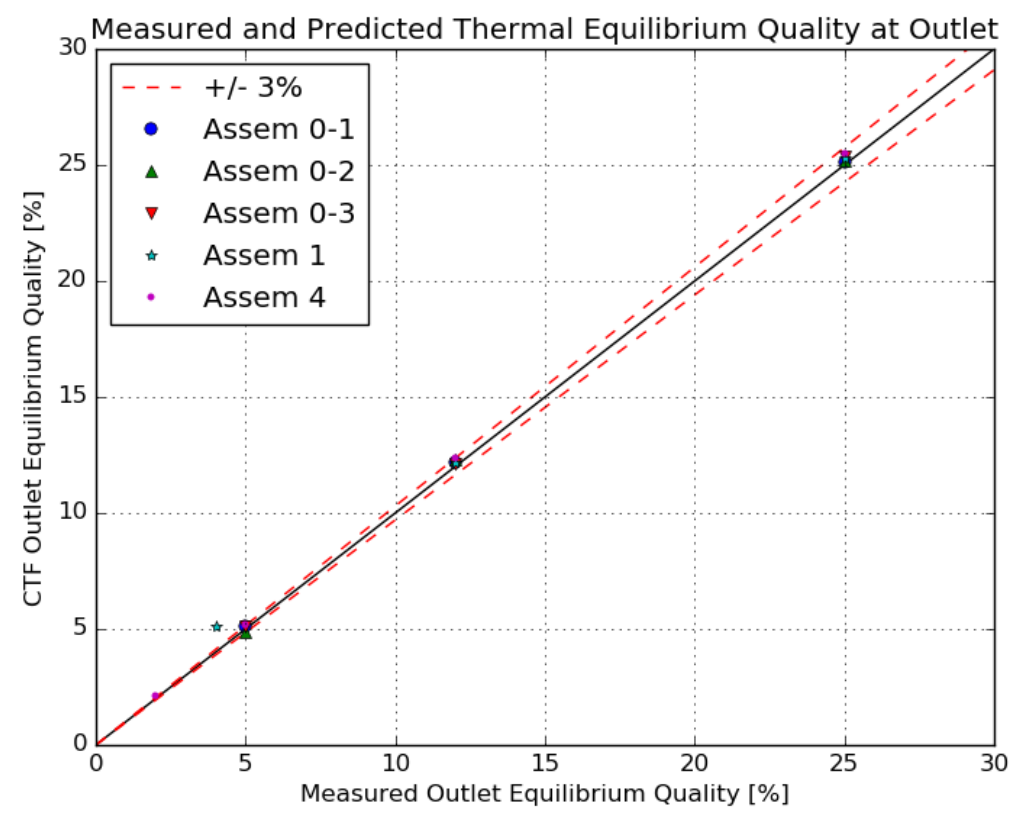

Figure 8.12: Comparison of measured and predicted bundle-averaged outlet thermal equilibrium quality.

phase slip differences can lead to discrepancies between the predicted and experimental void. It is also possible the experimental facility loses heat along the axial length, which therefore leads to a lower amount of void at the outlet compared with the perfectly adiabatic CTF simulation.

Figure 8.12 shows that exit equilibrium quality agrees with experimental values to within $+/-3 \%$ for most cases.

Before comparing specific channel outlet void measurements to CTF predictions, it is useful to check the symmetry of the experimental data. To do this, the measurements are grouped into categories set by the channel types, which are

1. corner channel,

2. side channel,

3. normal inner channel, and

4. inner channel touching unheated element.

The grouping is not perfect, as none of the assembly types are eighth-core symmetric, and the channels touching unheated elements have different flow areas and wetted perimeters and touch different numbers of heated rods. Regardless, the selected categorization leads to a simpler organization of the data, and we should expect variation in corner and side channels due to assembly asymmetry to be minimal because of their large distance from the asymmetric center 


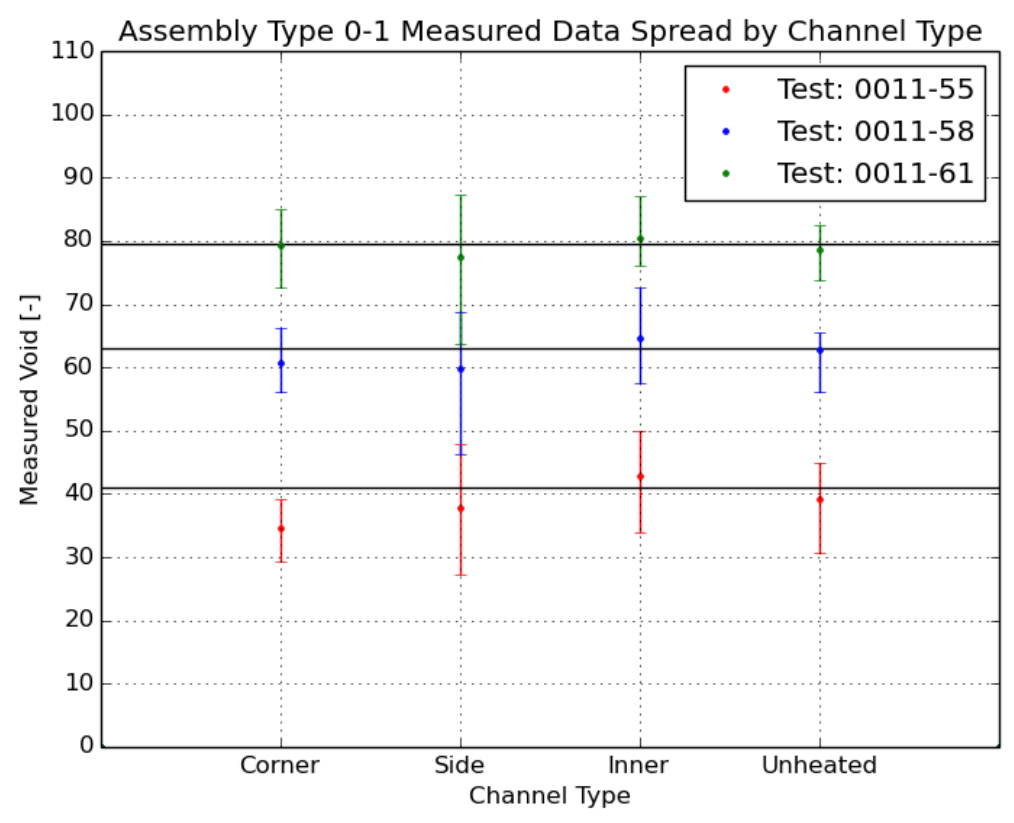

Figure 8.13: Summary of average void measurements for four channel categories in Assembly Type 0-1 experiments.

of the assembly. The check is done only for Assembly Types 0-1 and 0-2. Assembly Type 0-3 contains a large unheated section in the bundle that is asymmetric and close to the boundary rods, which we expect to lead to more significant asymmetry effects. Assembly Types 1 and 4 have non-uniform radial power distributions, which invalidate the selected data categorization.

Figures 8.13 and 8.14 give the mean void for each category as well as the spread of the data. The $\mathrm{x}$-axis contains the four categories. The $\mathrm{y}$-axis is the local subchannel void measurement. The dot gives the mean void for that category, and the error bars give the minimum and maximum void measurement for that category. The horizontal black lines give the bundle-average void at the outlet for each test in the figure. There are three tests shown in each figure (one figure per assembly type).

A few conclusions are drawn from the figures. First, the corner channel measurements tend to have the least scatter in measurement data, whereas the side channels tend to have the largest amount of scatter. Measurements for a given channel type tend to vary by as much as 10-20\%, which suggests that the measurement uncertainty may be larger than the $3 \%$ value quoted in the BFBT specification. Another observation is that the void drift phenomena becomes evident when comparing mean void to bundle-average void for all experiments. The corner and side voids are typically below the bundle-average value, whereas the inner channel void is typically above the bundle-average void. As expected, the channels that touch unheated elements often have the lowest void. The void in unheated channels is lowest for Assembly Type 0-2, which has two heater rods disabled in the center of the assembly. It is additionally noted that this trend is more exaggerated for test cases with lower total void content. As total void content increases, 


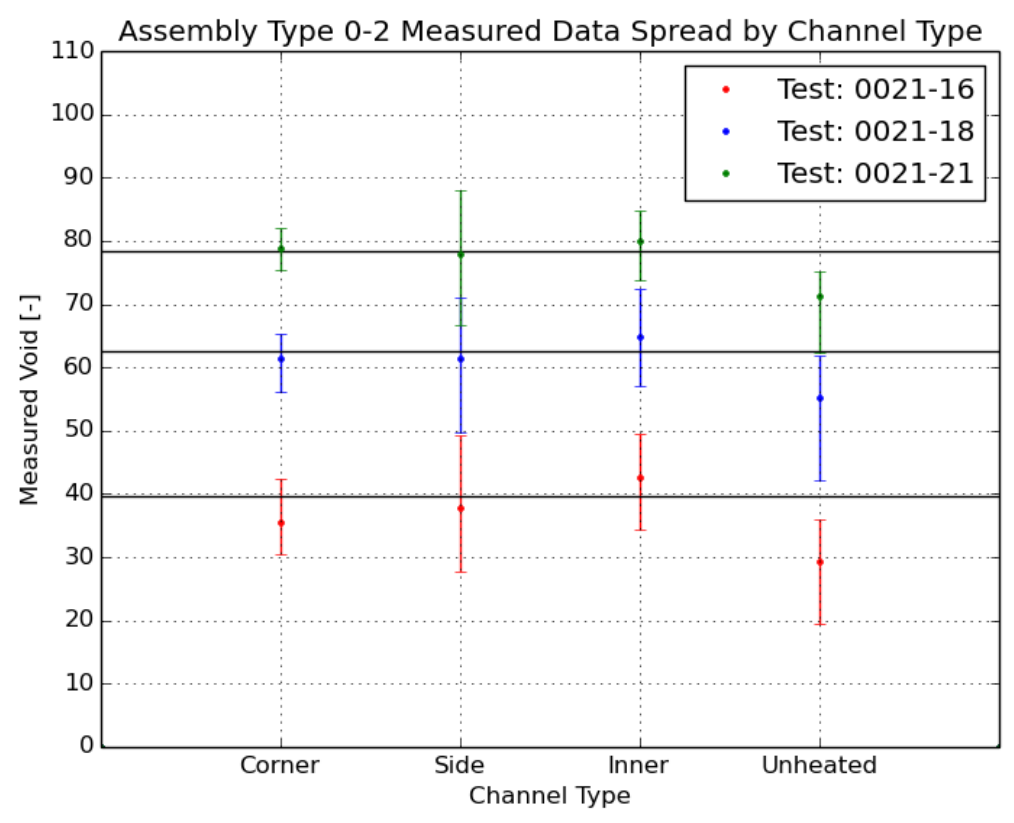

Figure 8.14: Summary of average void measurements for four channel categories in Assembly Type 0-2 experiments.

the outlet void distribution tends to be more uniform. Although conclusions on data symmetry cannot be drawn for the remaining assembly types, similar plots are shown in Figures 8.15-8.17 to demonstrate the void drift trend for the other assembly types.

Figure 8.18 gives an overview of the measured versus predicted subchannel void measurements for all tests. The data is grouped by assembly type, so multiple experiments are included in each dataset. The data shows that there is an overprediction of void; data is frequently over the $+10 \%$ line but never falls below the $-10 \%$ line. Also, the data appears to form into horizontal "stripes." This is due to the asymmetric behavior and spread of the experimental data compared with the symmetric behavior of the CTF predictions. The RMSE of all data is $7.95 \%$ compared with experimental data.

A second simulation of the cases is performed with the droplet field enabled to show the effect. Results for all tests are shown in Figure 8.19.

More insight is gained by breaking the data comparison into the four aforementioned channel categories. This is shown in Figures 8.20 (no droplets) and 8.21 (with droplets) for the corner type channels, Figures 8.22 (no droplets) and 8.23 (with droplets) for the side type channels, Figure 8.24 (no droplets) and 8.25 (with droplets) for the normal inner type channels, and Figure 8.26 (no droplets) and 8.27 (with droplets) for the inner channels touching unheated elements.

In every category, enabling the droplet field causes a degradation in prediction accuracy of a few percentage points. The worst predictions are for the channels that touch unheated elements. CTF tends to overpredict the void in these channels. RMSE for the unheated channels is $11.68 \%$. 


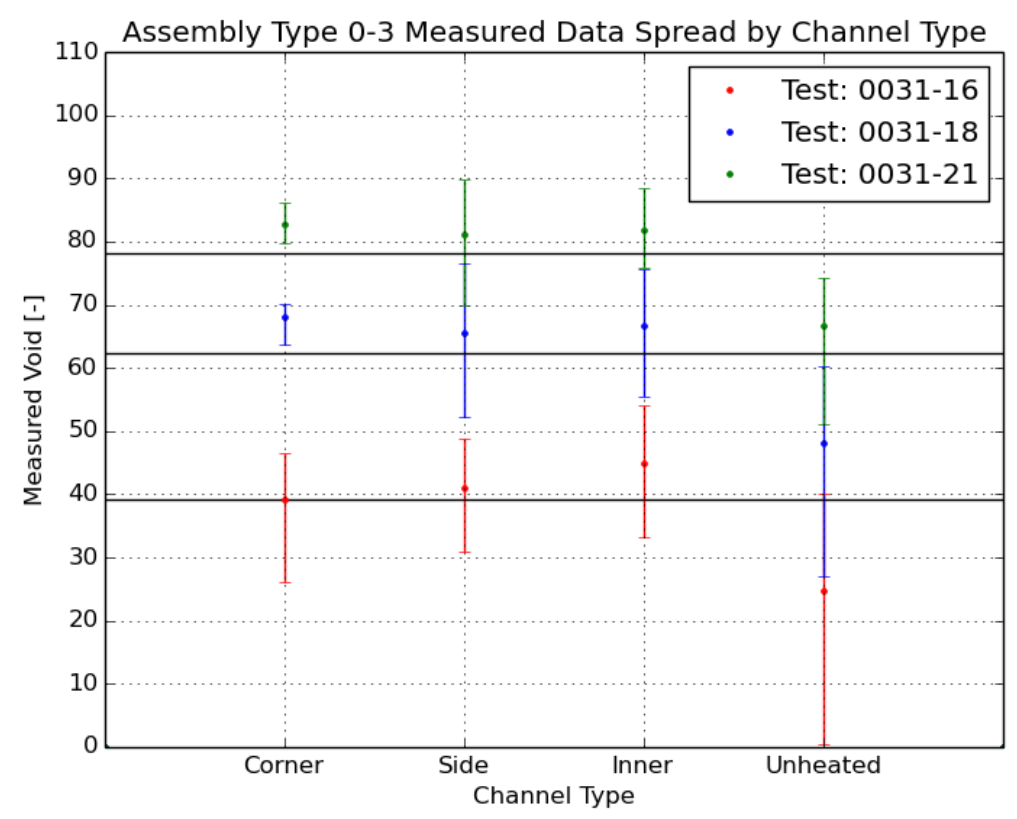

Figure 8.15: Summary of average void measurements for four channel categories in Assembly Type 0-3 experiments.

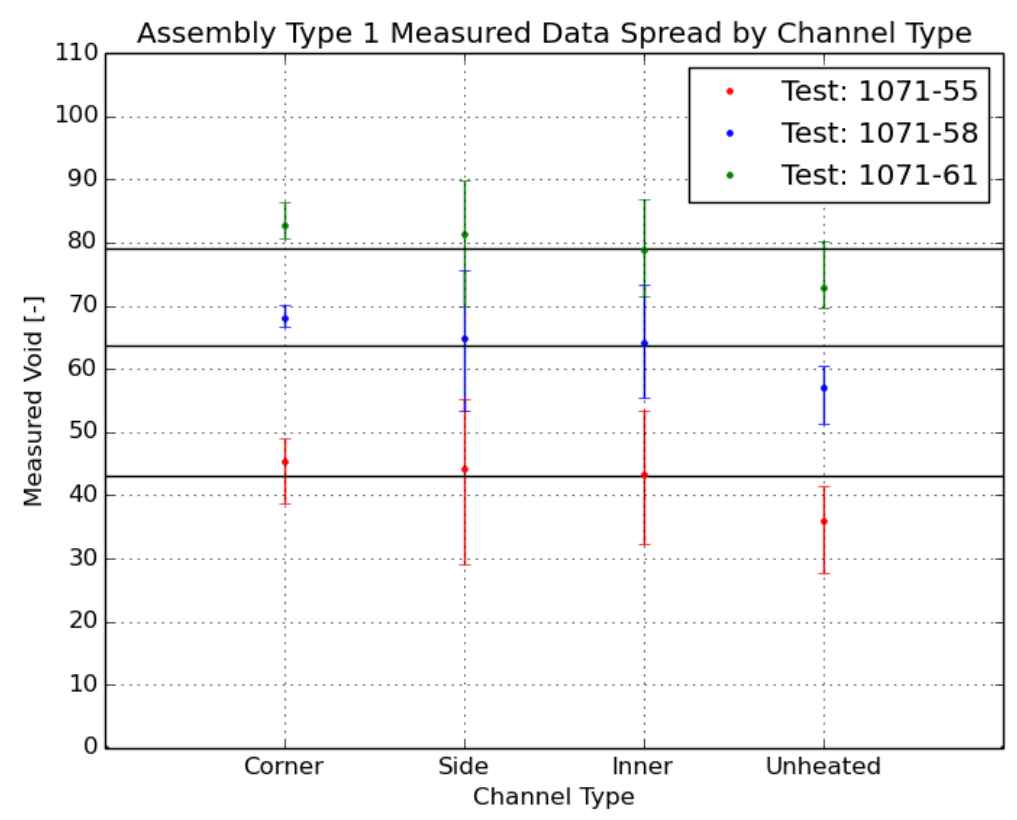

Figure 8.16: Summary of average void measurements for four channel categories in Assembly Type 1 experiments. 


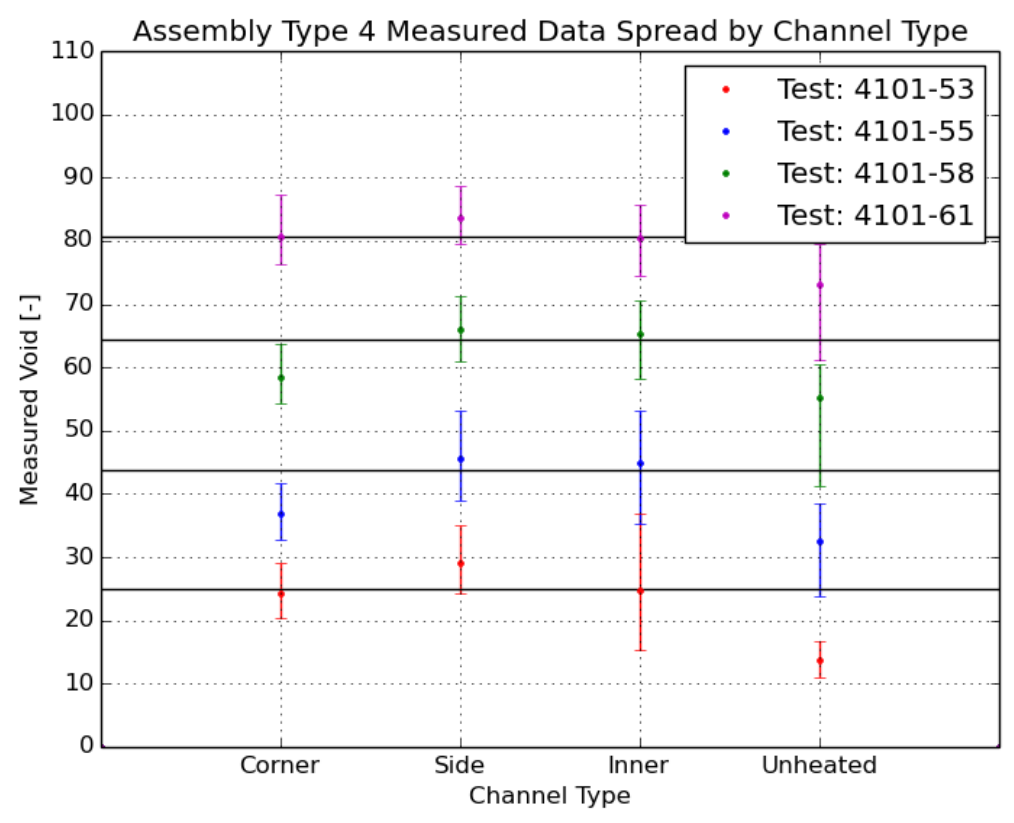

Figure 8.17: Summary of average void measurements for four channel categories in Assembly Type 4 experiments.

The inner channels, on the other hand, almost alway fall within the $\pm 10 \%$ bands in the plot. The RMSE for this channel type is only $5.52 \%$. As was observed in the GE $3 \times 3$ validation study, the error in the boundary channels tends to be higher. The corner channel RMSE is $7.61 \%$ and the side channel RMSE is $9.05 \%$. However, it was found in the GE $3 \times 3$ study that the corner channel exhibited the largest error compared with experimental data. The tendency for CTF to overpredict void near the unheated structures has been observed for other codes used to simulate this data in the OECD/NRC Benchmark [20].

It is useful to look into the effects of the CTF void drift model. The cases are rerun with the void drift model disabled (single- and two-phase turbulent mixing is still enabled and set to the previous values). The droplet model is disabled from this point forward. Figure 8.28 shows all measurement data compared with CTF predictions when the void drift model is disabled. As shown in the figure, the RMSE increases by about 1 percentage point. Figures $8.29-8.32$ show the results broken down by channel category when the void drift model is disabled. The results show that the corner type channel witnesses the largest increase in error, from $7.61 \%$ to $11.56 \%$. The side channel also sees an increase in error, from $9.05 \%$ to $11.82 \%$. The channels touching an unheated element experience practically no change in error, from $11.68 \%$ to $11.56 \%$ and the inner type channels actually see a slight improvement in error, dropping from $5.52 \%$ to $4.62 \%$. Results for the four different groupings and three different parametric studies are shown in Table 8.1 .

As it has been shown that there is a tendency for CTF to globally over-predict measured void data, it is important to use the data to look at trends. In other words, the void drift phenomena 


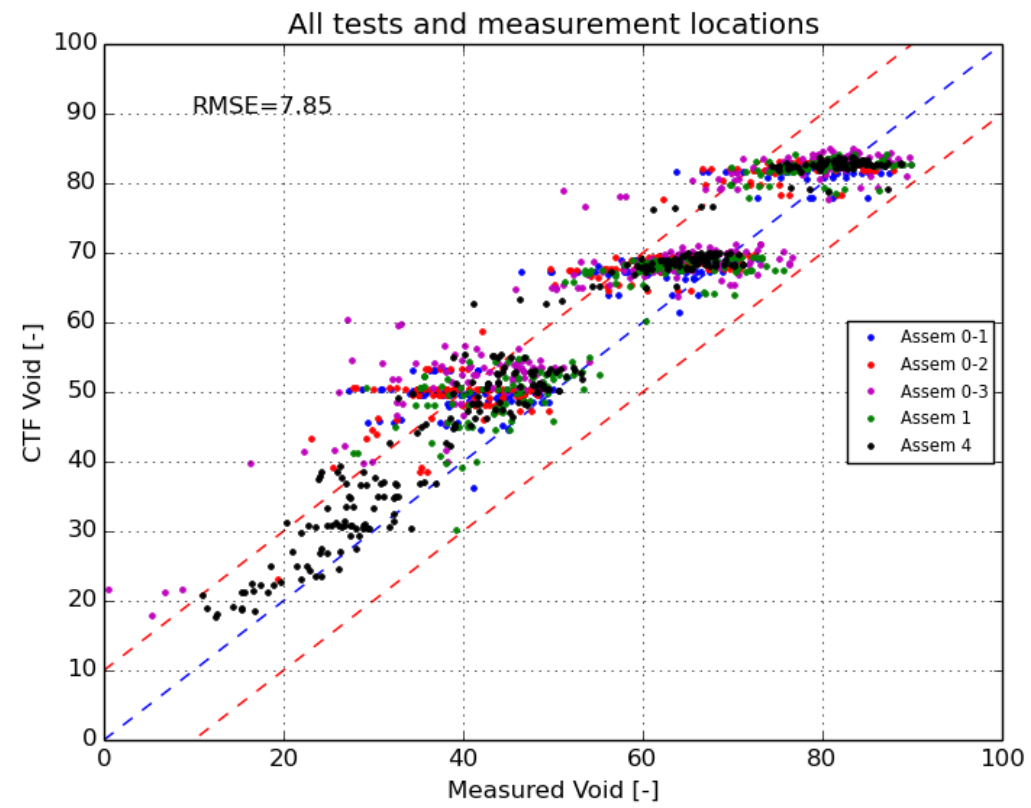

Figure 8.18: Comparison of CTF predictions and measurements of subchannel void for all BFBT void distribution tests.

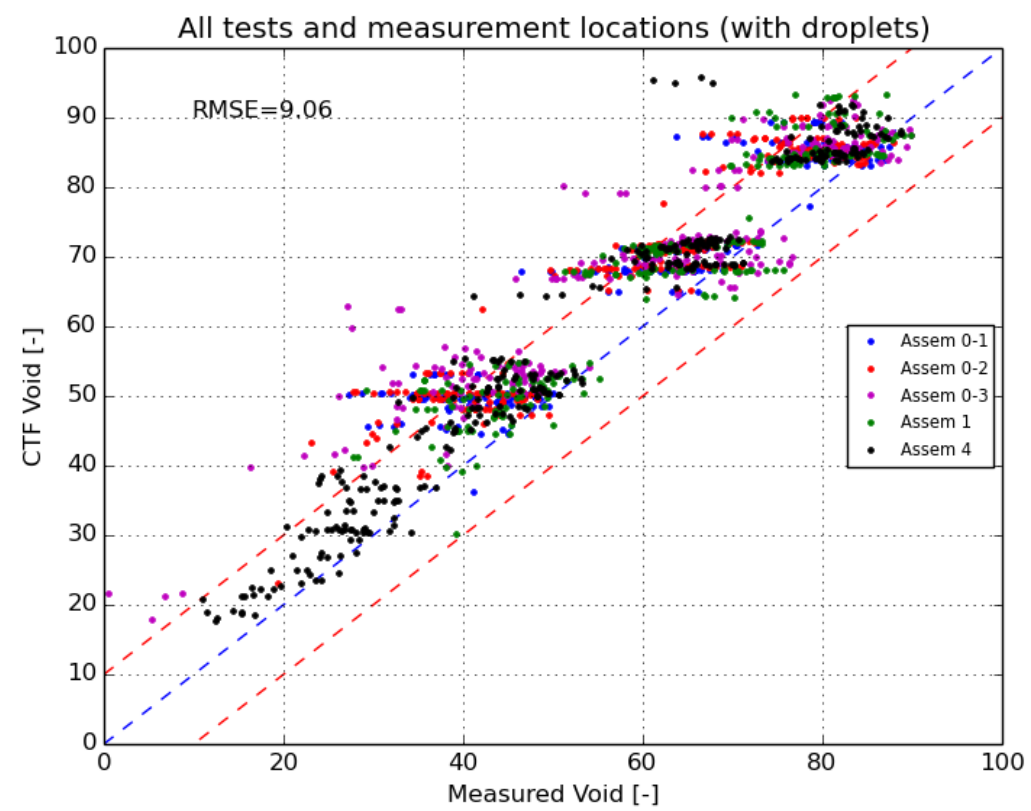

Figure 8.19: Comparison of CTF predictions and measurements of subchannel void for all BFBT void distribution tests (with droplets). 


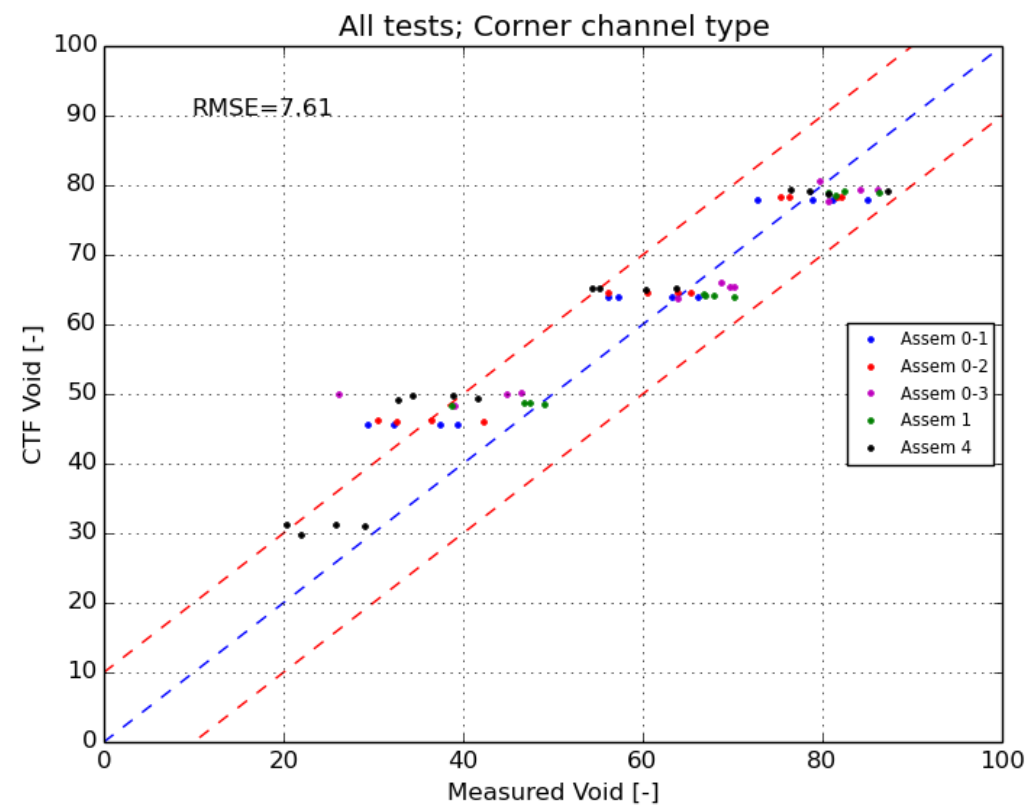

Figure 8.20: Comparison of CTF predictions and measurements of subchannel void for cornertype channels in all modeled BFBT void distribution tests.

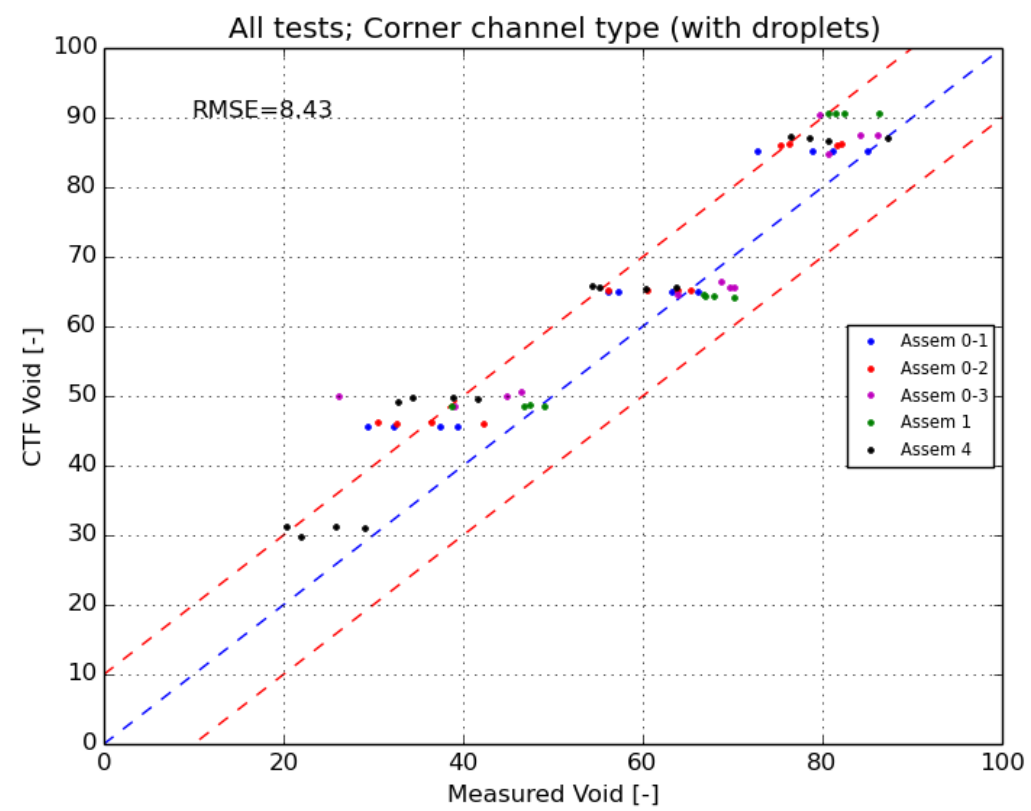

Figure 8.21: Comparison of CTF predictions and measurements of subchannel void for cornertype channels in all modeled BFBT void distribution tests (with droplets). 


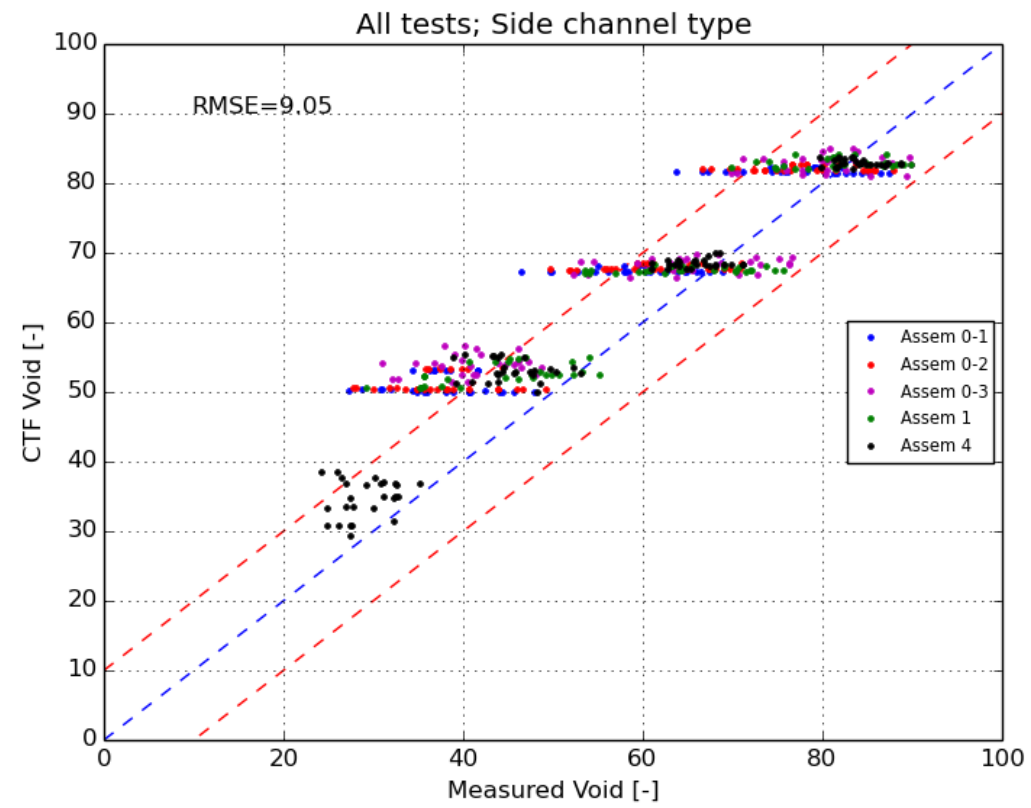

Figure 8.22: Comparison of CTF predictions and measurements of subchannel void for side-type channels in all modeled BFBT void distribution tests.

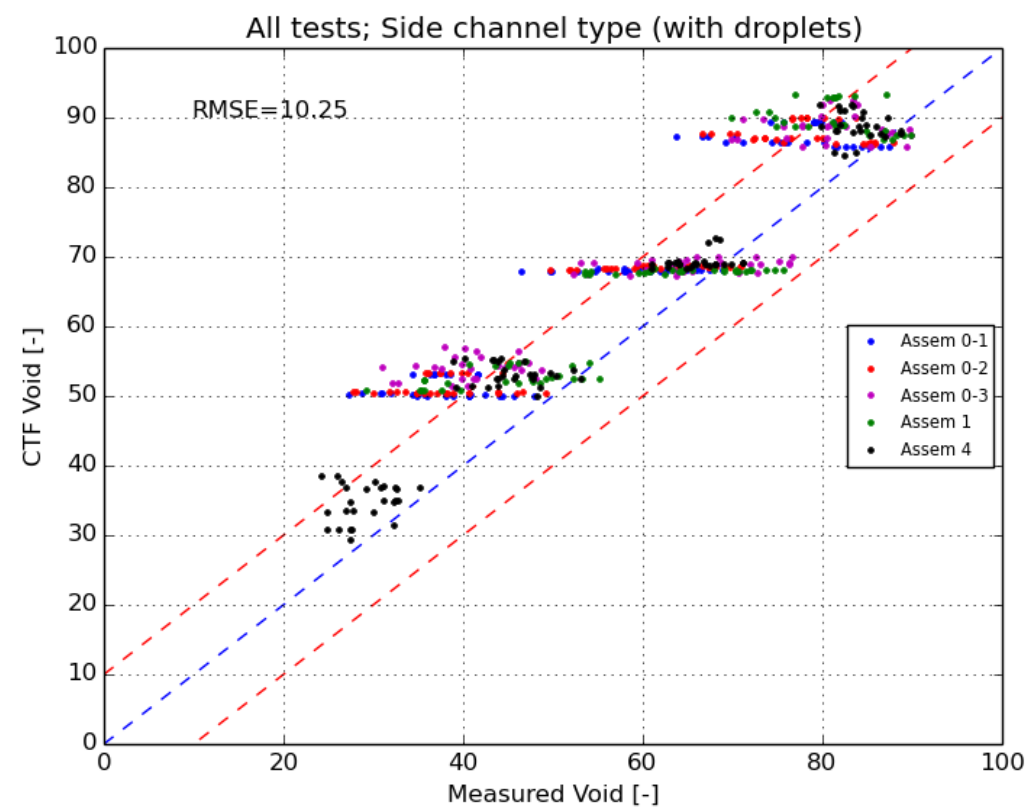

Figure 8.23: Comparison of CTF predictions and measurements of subchannel void for side-type channels in all modeled BFBT void distribution tests (with droplets). 


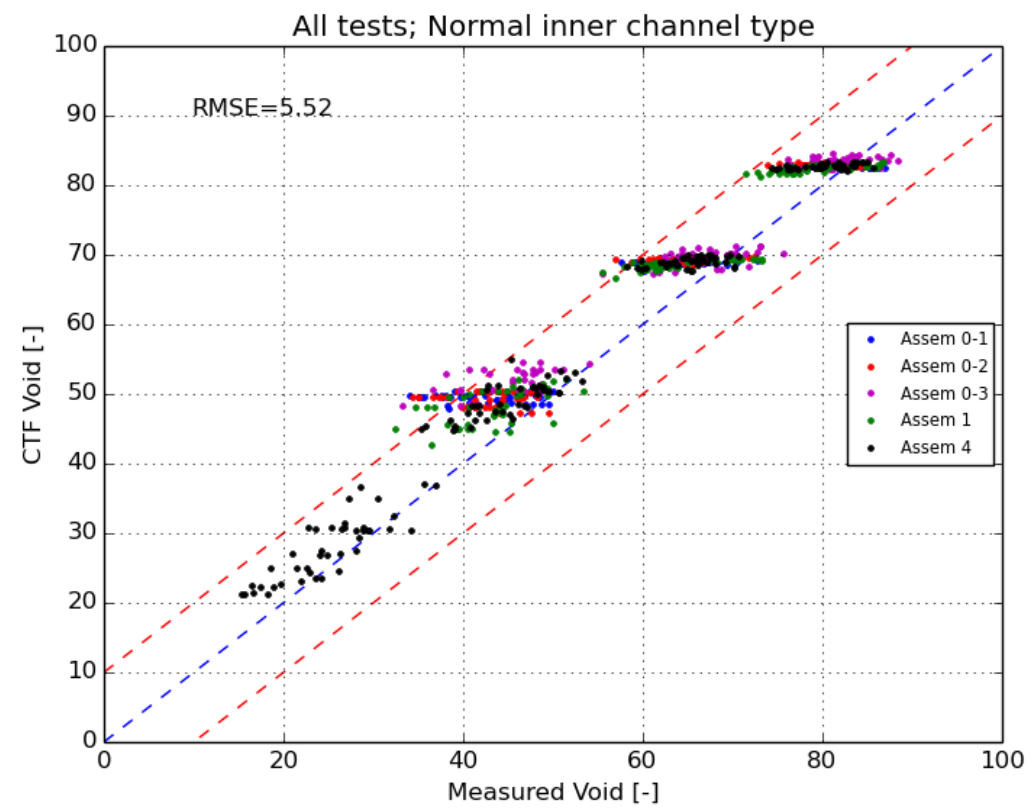

Figure 8.24: Comparison of CTF predictions and measurements of subchannel void for inner-type channels in all modeled BFBT void distribution tests.

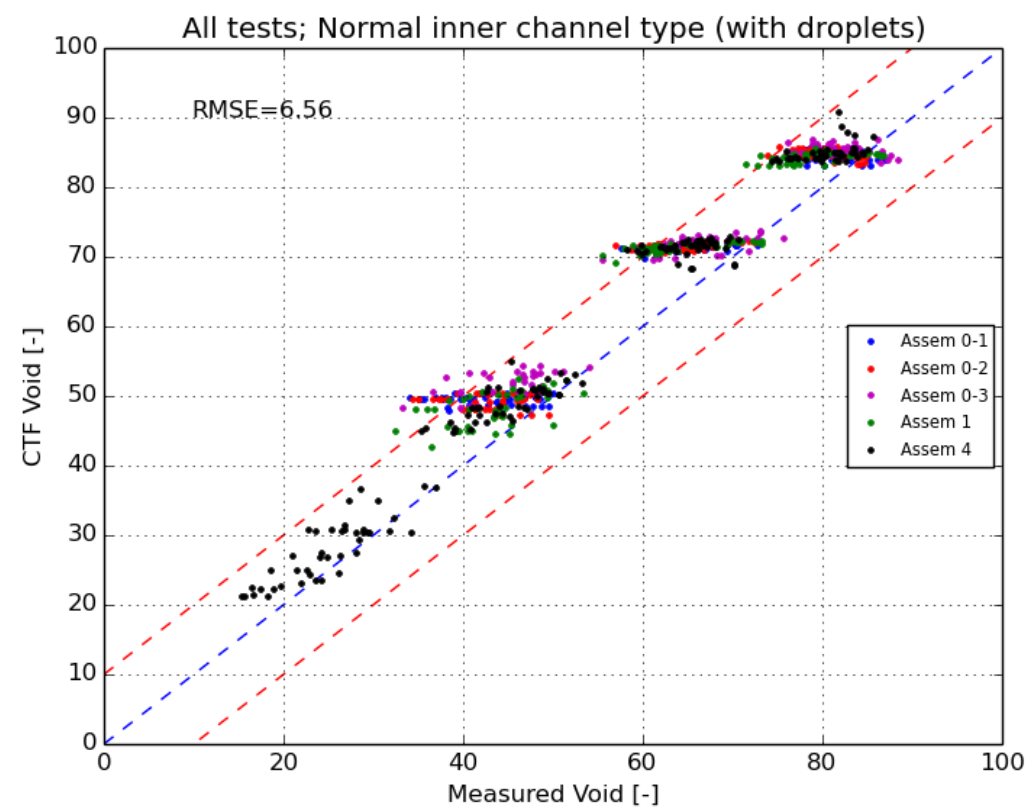

Figure 8.25: Comparison of CTF predictions and measurements of subchannel void for inner-type channels in all modeled BFBT void distribution tests (with droplets). 


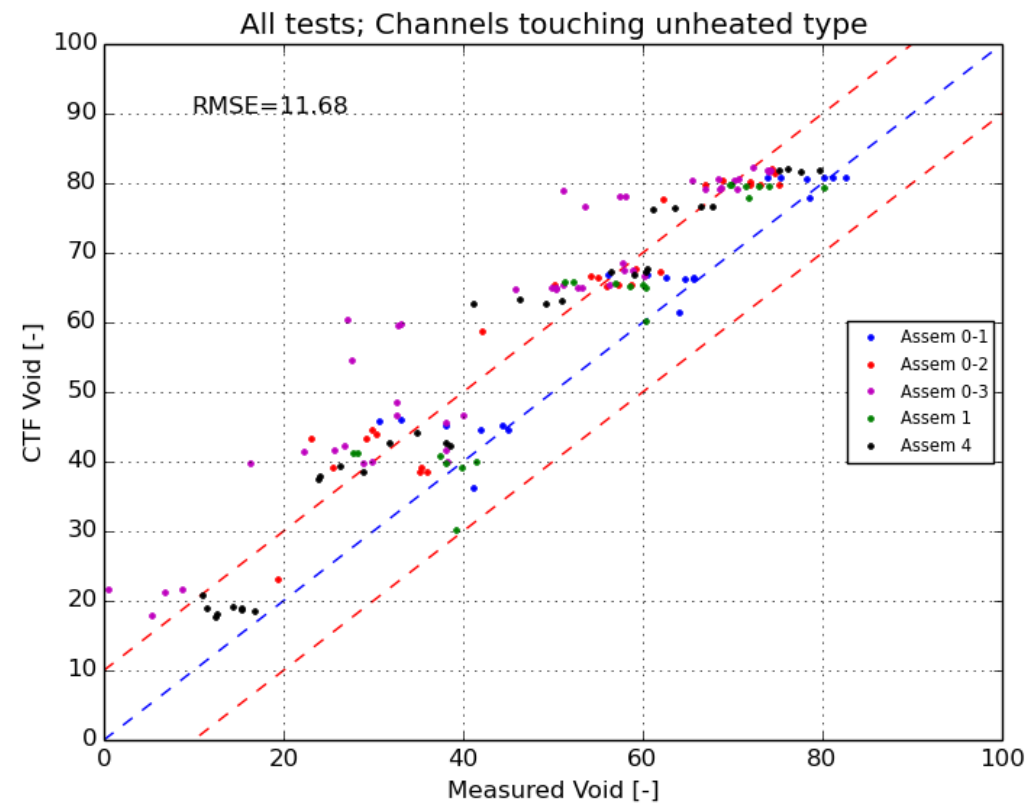

Figure 8.26: Comparison of CTF predictions and measurements of subchannel void for unheatedtype channels in all modeled BFBT void distribution tests.

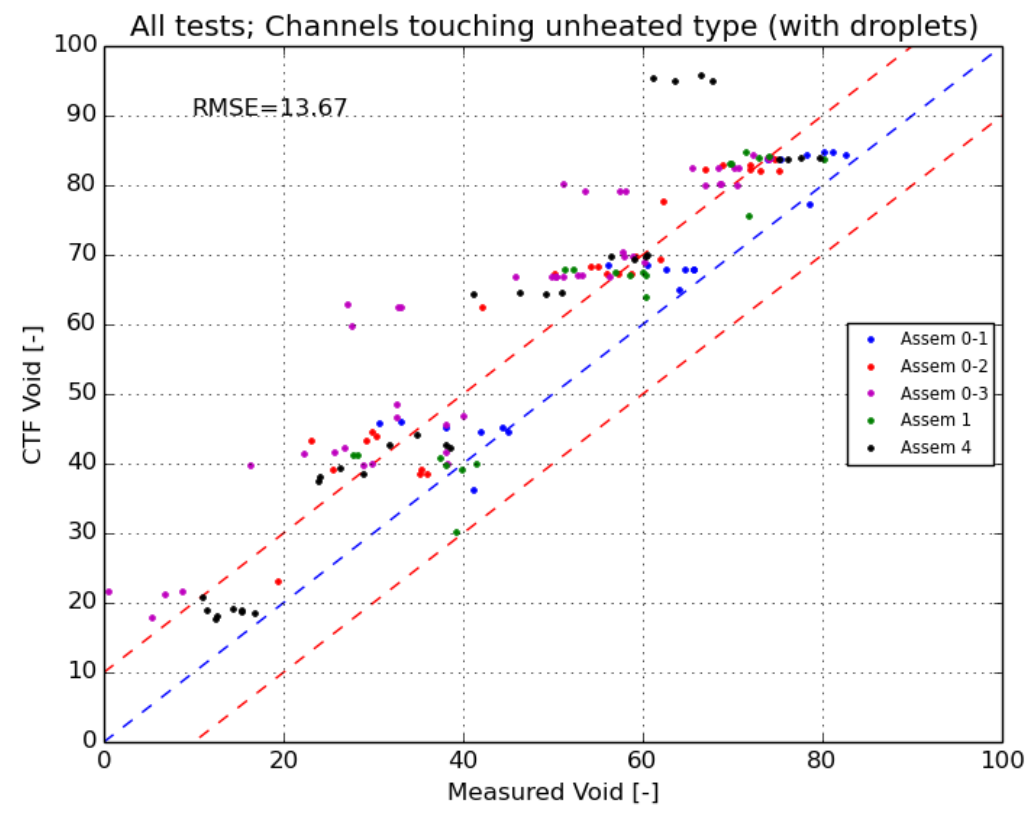

Figure 8.27: Comparison of CTF predictions and measurements of subchannel void for unheatedtype channels in all modeled BFBT void distribution tests (with droplets). 


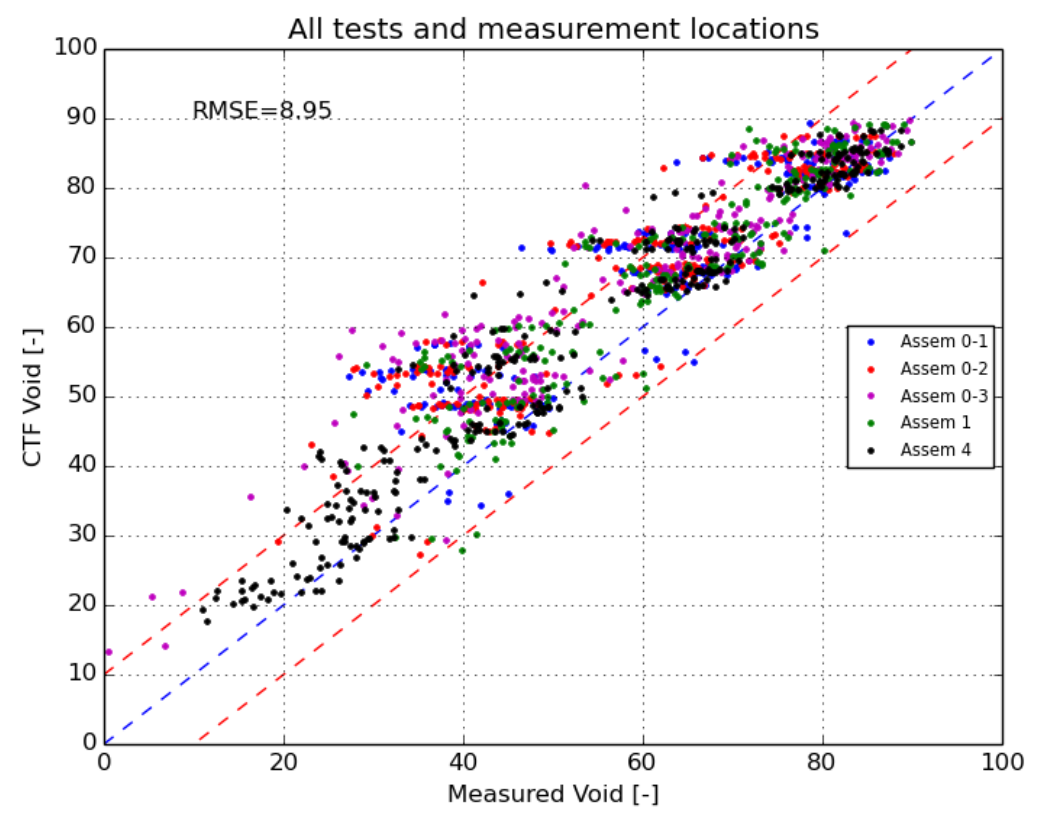

Figure 8.28: Comparison of CTF predictions and measurements of subchannel void for all channels in all modeled BFBT void distribution tests when void drift model is disabled.

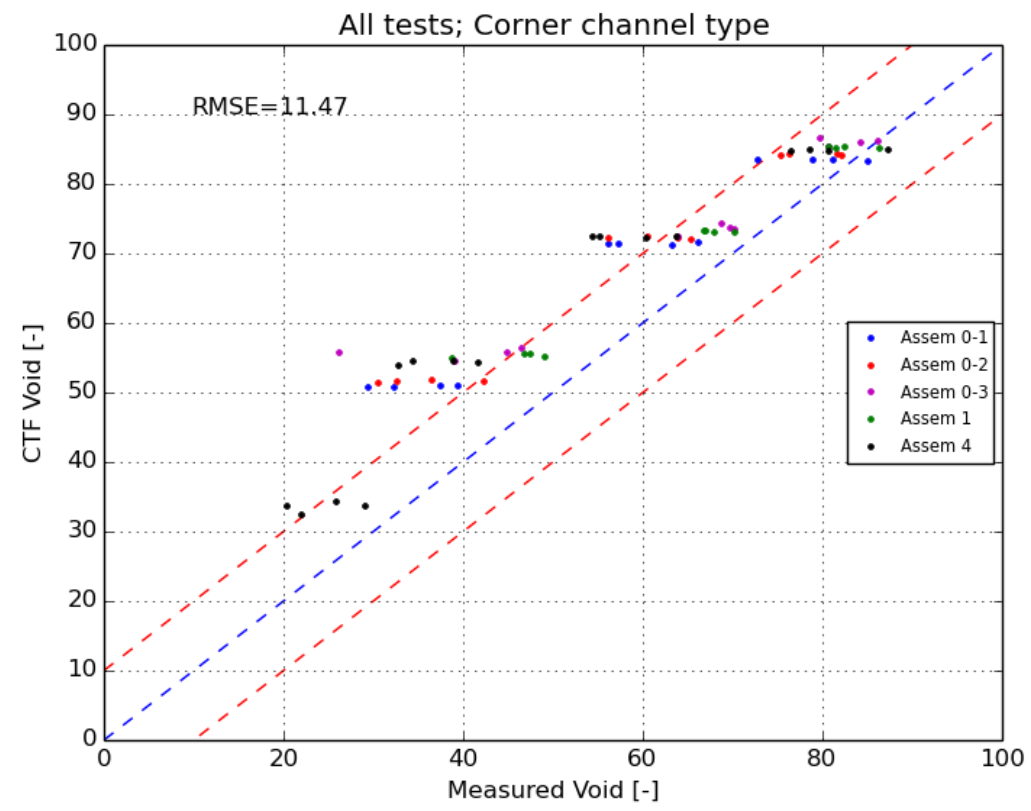

Figure 8.29: Comparison of CTF predictions and measurements of subchannel void for corner channels in all modeled BFBT void distribution tests when void drift model is disabled. 


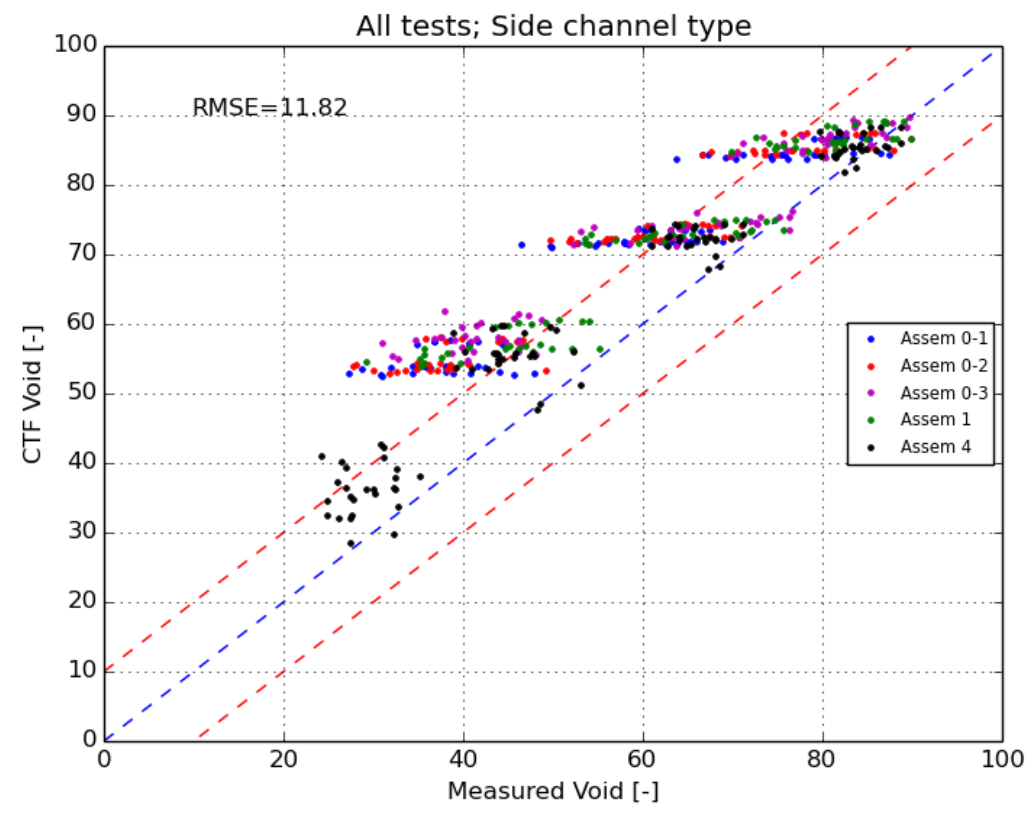

Figure 8.30: Comparison of CTF predictions and measurements of subchannel void for side channels in all modeled BFBT void distribution tests when void drift model is disabled.

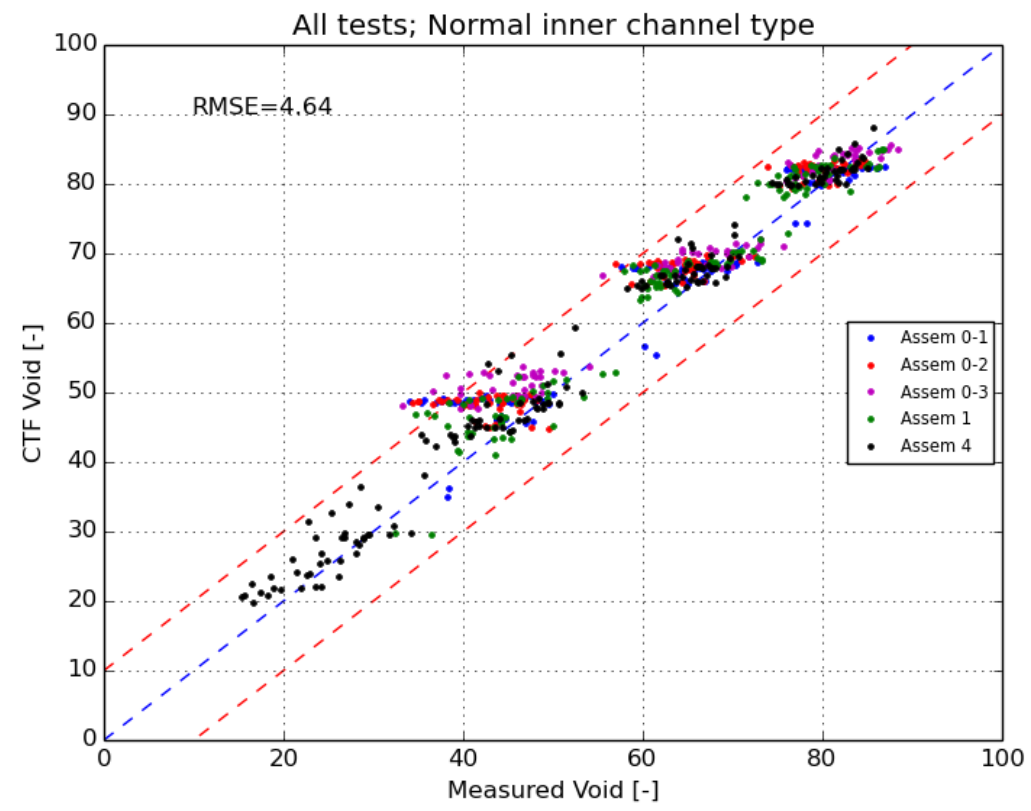

Figure 8.31: Comparison of CTF predictions and measurements of subchannel void for inner channels in all modeled BFBT void distribution tests when void drift model is disabled. 


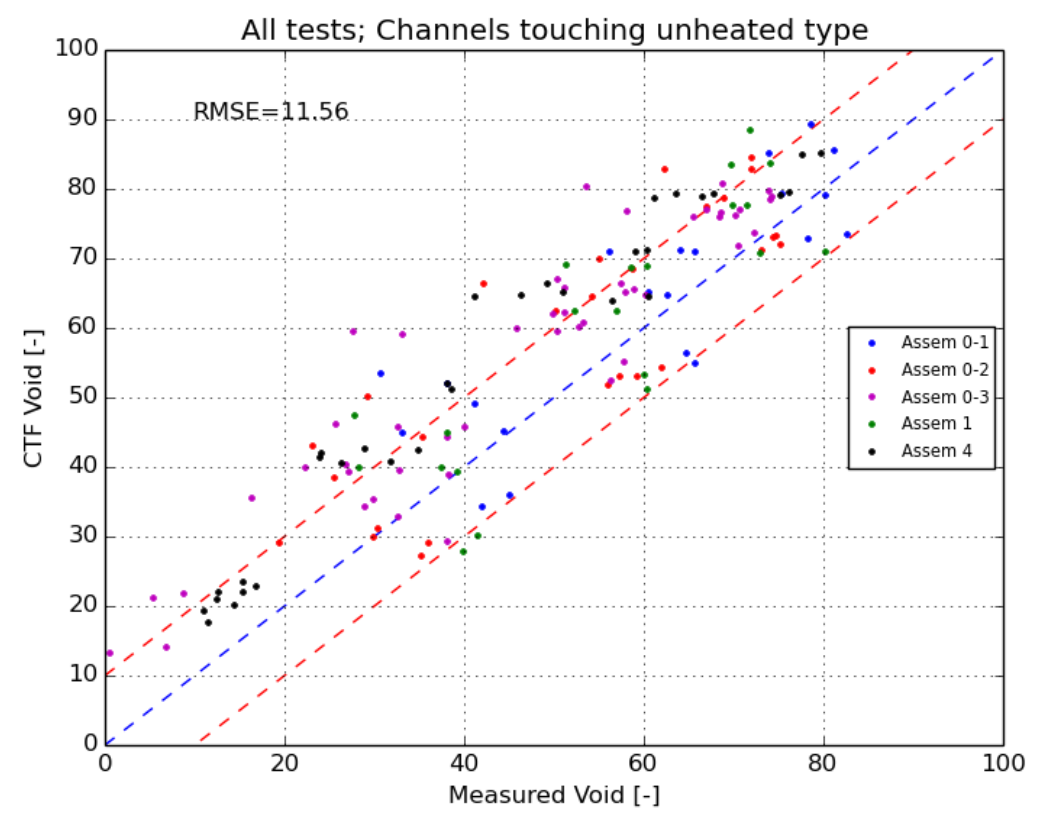

Figure 8.32: Comparison of CTF predictions and measurements of subchannel void for unheated channels in all modeled BFBT void distribution tests when void drift model is disabled.

Table 8.1: Summary of the CTF predictions of BFBT void distribution cases

\begin{tabular}{|c|c|c|c|}
\hline $\begin{array}{l}\text { Channel } \\
\text { Group }\end{array}$ & $\begin{array}{r}\text { No Drops } \\
\text { With Void Drift }\end{array}$ & $\begin{array}{l}\text { With Drops } \\
\text { With Void Drift }\end{array}$ & $\begin{array}{r}\text { No Drops } \\
\text { No Void Drift }\end{array}$ \\
\hline Corner & 7.61 & 8.43 & 11.47 \\
\hline Side & 9.05 & 10.25 & 11.82 \\
\hline Inner & 5.52 & 6.56 & 4.64 \\
\hline Unheated & 11.68 & 13.67 & 11.56 \\
\hline All & 7.85 & 9.06 & 8.95 \\
\hline
\end{tabular}




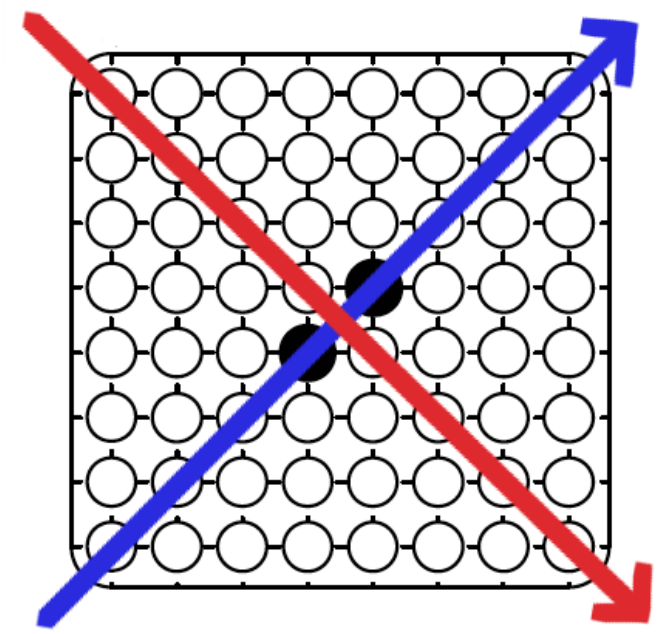

Figure 8.33: Example of diagonal lines from where subchannel void data is extracted.

causes void to migrate from smaller area channels to higher area channels. It is important to look for this behavior in the simulations. The void drift model should be enabled and disabled to observe the impact of the model on results. To do this, void data is visualized over the diagonals of each assembly. Figure 8.33 gives an example of the two diagonal lines that are struck through the assemblies. The example is given for Assembly Type 0-1, but the process is the same for all five assembly types. Note that the red line goes from the northwest to the southeast, and the blue line goes from the southwest to the northeast. The experimental data along these lines will be shown with dots, and the CTF predictions will be shown with lines. The red dots and lines will be for the northwest to southeast diagonal, and the blue dots and lines will be for the southwest to northwest diagonal.

Figure 8.34 shows results for Test 0011-55, which is from Assembly Type 0-1. Figure 8.35 shows the same results, but with the void drift model disabled in the CTF simulation. In both cases, the CTF results are fairly symmetric. The red line has slightly different behavior because the center of the bundle has two guide tubes that lead to an asymmetric rod placement. In both cases, CTF captures the reduction in void in the center of the model because of the presence of the unheated guide tubes. However, the reduction is not as severe as it is in the experimental results.

Additionally, the experimental results see a jump up in void for the center channel. This behavior is likely due to the design of the spacer grid, which offers no flow resistance to the central subchannel. Despite the central subchannel having a smaller hydraulic diameter than surrounding channels (and thus a higher frictional pressure drop), the lack of a form loss allows the velocity to climb higher than its neighbors, which causes the higher velocity void to seek the path of least flow resistance and migrate into the center subchannel.

With void drift disabled, CTF sees a larger drop in void in the center channel than when void drift is enabled. Void increasing in the center subchannel is the correct behavior due to the lack 


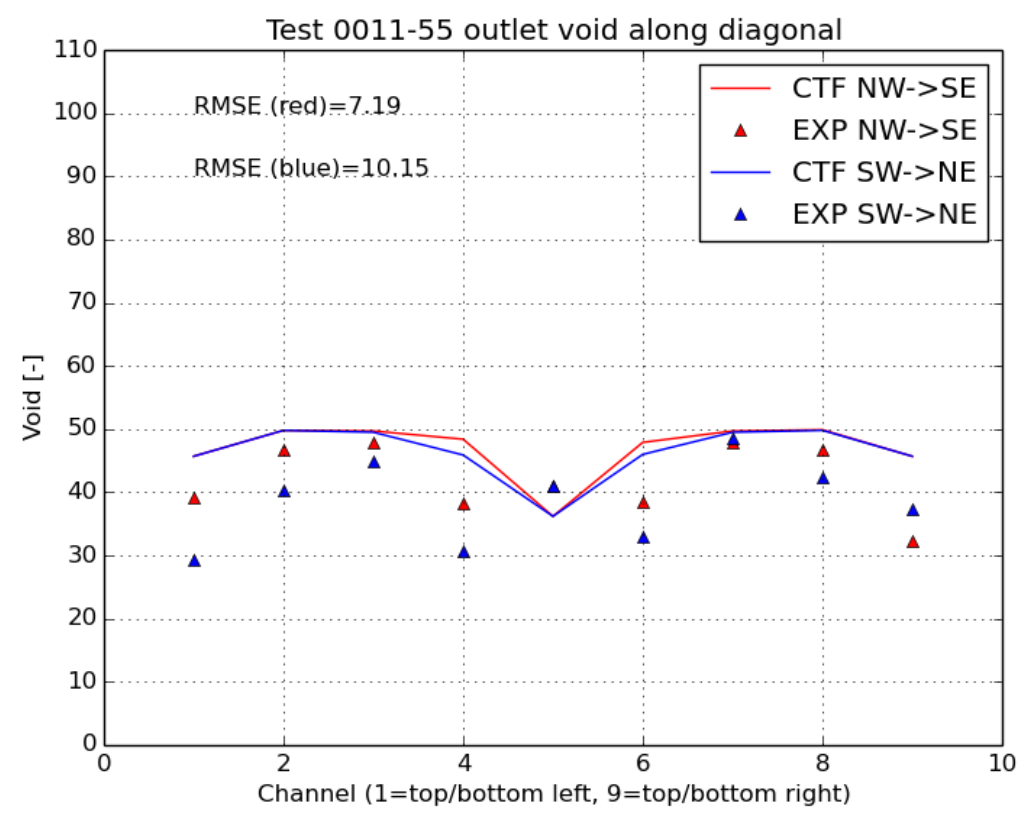

Figure 8.34: Measured and predicted void along diagonals of Test 0011-55.

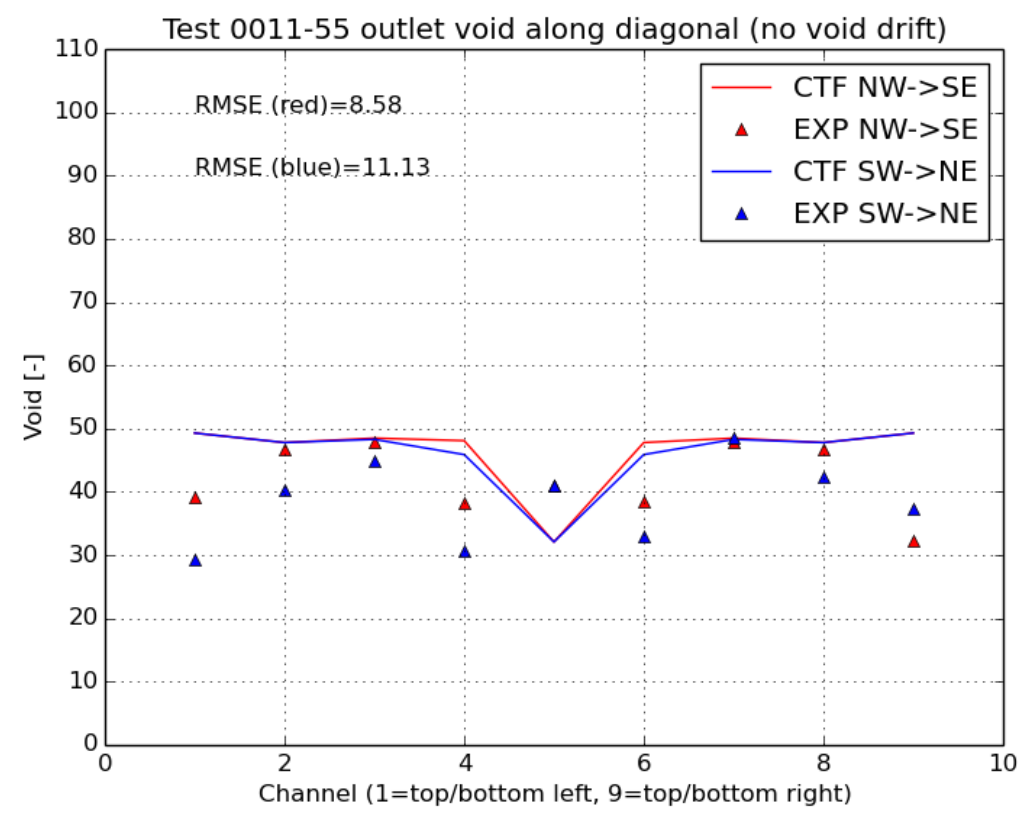

Figure 8.35: Measured and predicted void along diagonals of Test 0011-55 with void drift disabled. 
of a form loss in that channel. The CTF void drift model acts to move void from lower to higher velocity subchannels, so turning the void drift model on causes void to migrate from the larger area channels (which have lower velocity) into the center channel (which has higher velocity).

When void drift is enabled, the trend of void decreasing in the corner channels is predicted. When void drift is disabled, the void is highest in the corner channels, which is incorrect. While the trend is predicted correctly, the magnitude of the drop is less in CTF than in the experimental results. It is possible that the experimental facility walls are not perfectly adiabatic, which may explain the over-prediction of void in CTF. Overall, there is an improvement of about 1 percentage point for the RMSE of both lines of CTF predictions when compared to the experimental data.

As has been previously noted, the void drift effect becomes less severe at higher overall bundle void content. Test 0011-61 is also run in Assembly Type 0-1; however, it is run at a much higher power, leading to a bundle average void that is about double that of Test 0011-55. Figure 8.36 shows the void distribution on the diagonals with void drift enabled. Figure 8.37 shows the same data, but with void drift disabled in the CTF simulations.

It is observed that the CTF results, with void drift enabled, compare very nicely to experimental data because the void drift phenomena is not as severe. The RMSE is between 4 and 6 percentage points lower than the lower void case, Test 0011-55. Disabling the void drift model actually leads to an improvement in one of the diagonals when comparing to experimental data due to the large asymmetry in the corner results.

The low-void test from Assembly 0-2, which has four unheated rods in the bundle center, is shown in Figure 8.38 with void drift enabled and in Figure 8.39 with void drift disabled. Similar trends are observed, but it is noted that void content decreases more in the center than it did in Assembly 0-1 due to the disabling of the two center heater rods. As in the case of Assembly Type 0-1, the void drift effect is less prominent in the higher void case, which is shown in Figure 8.40 (with void drift) and Figure 8.41 (without void drift). Note, though, that enabling the void drift model in CTF allows a great deal of void to migrate into the center of the bundle, which is unrealistic compared with the experimental results.

Test case 0031-16 is run in Assembly Type 0-3, which has seven unheated rods and two guide tubes in a position that is offset from the center of the bundle. This leads to an asymmetric distribution in the void. Figure 8.42 shows the CTF results with void drift on, and Figure 8.43 shows the results with void drift disabled. The red line is shifted to the right because of the additional unheated rods in the southeast quadrant of the assembly. This trend matches the data (the red dots also shift to the right). However, lowest void appears in the channel at Index 6 in the plots in the experimental data. CTF predicts the lowest void at Index 5 (center of the bundle). This is the case whether void drift is enabled or disabled, so the turbulent mixing must be driving void out of the center channel due to the higher velocity in the center channel. Because of the better-predicted void in the unheated region with void drift disabled, the RMSE is slightly better when void drift is disabled. A similar result is found for the higher-void case of Assembly 0-3; the void drift model gets the corner channels right but causes too much void to drift into the unheated region of the bundle. Figures 8.44 and 8.45 show this behavior.

Tests from Assembly Type 1 have a non-uniform axial and radial power distribution. The assembly has two guide tubes in the center like Assembly Type 0-1. The low-void case shows slightly better agreement with the data with void drift enabled (Figure 8.46) compared with void 


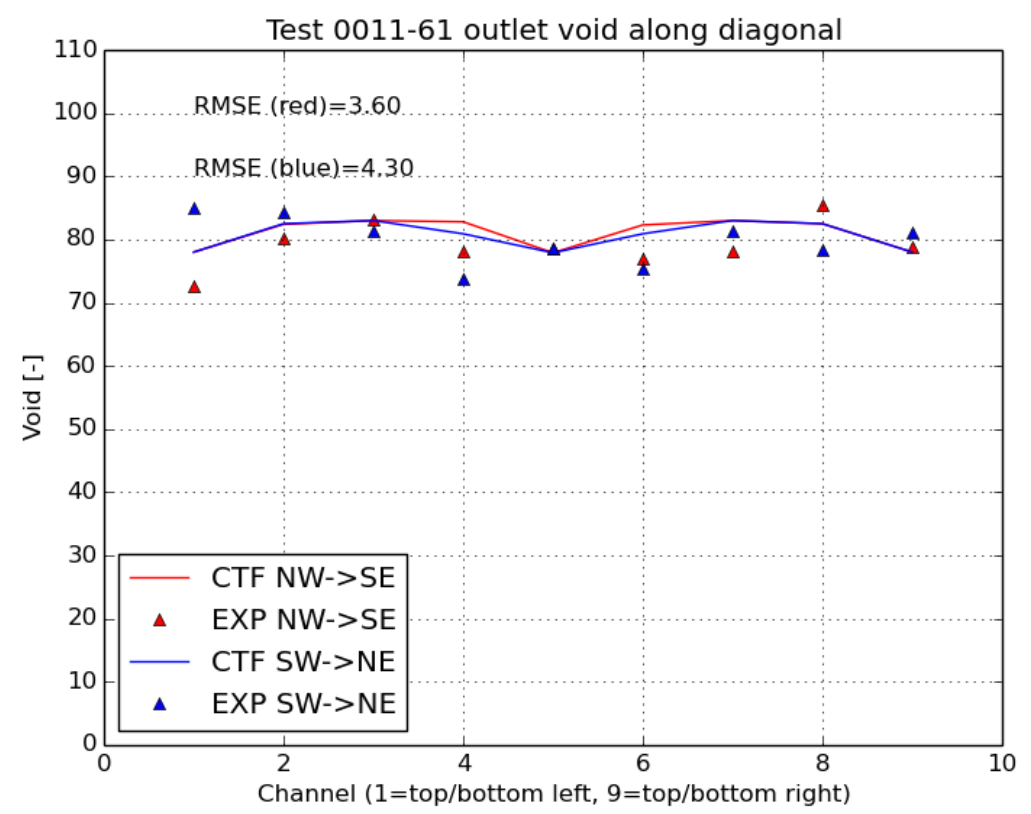

Figure 8.36: Measured and predicted void along diagonals of Test 0011-61.

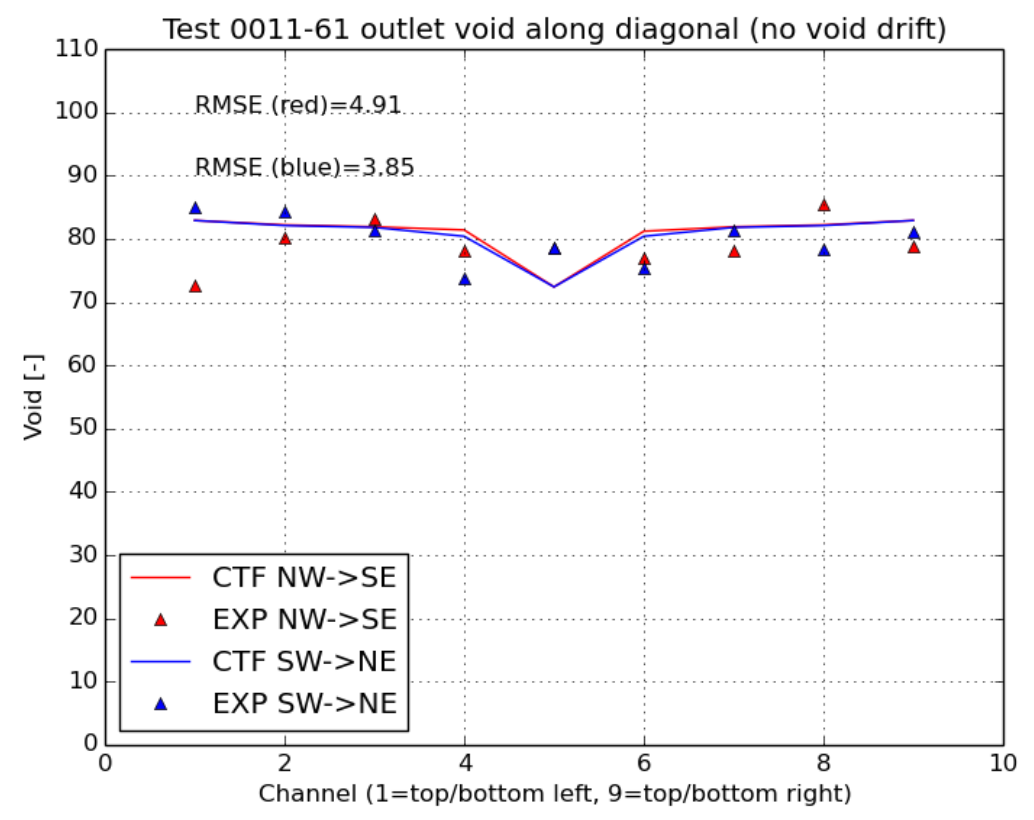

Figure 8.37: Measured and predicted void along diagonals of Test 0011-61 with void drift disabled. 


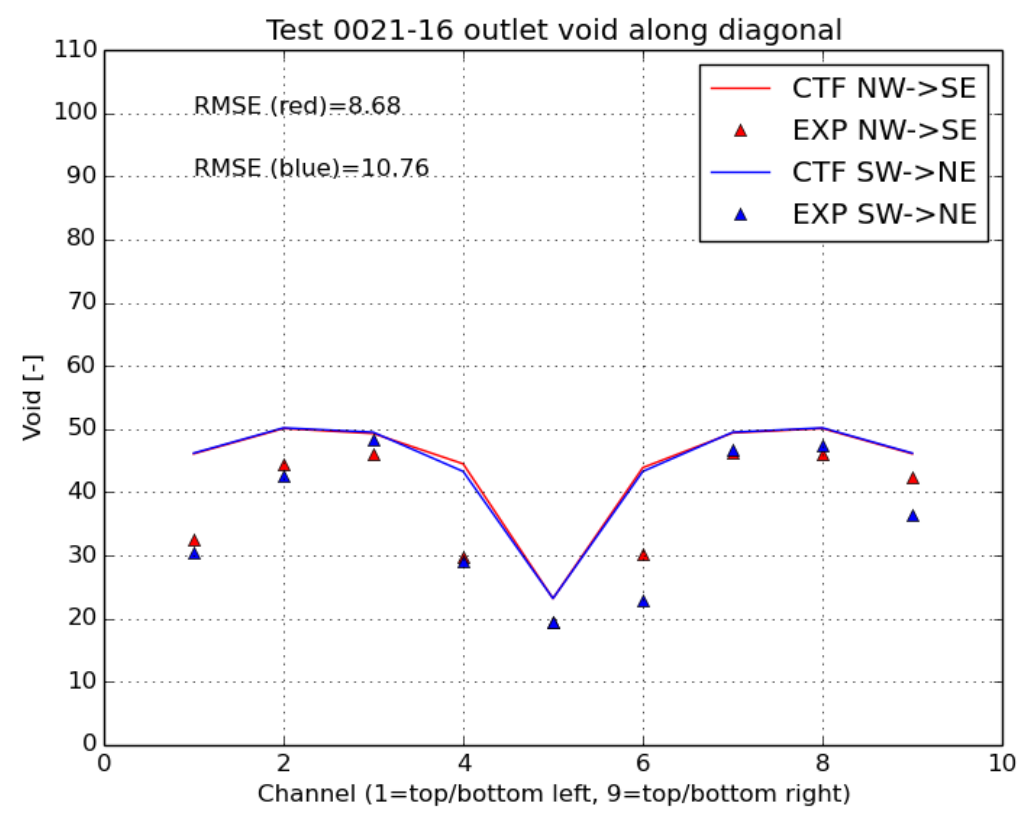

Figure 8.38: Measured and predicted void along diagonals of Test 0021-16.

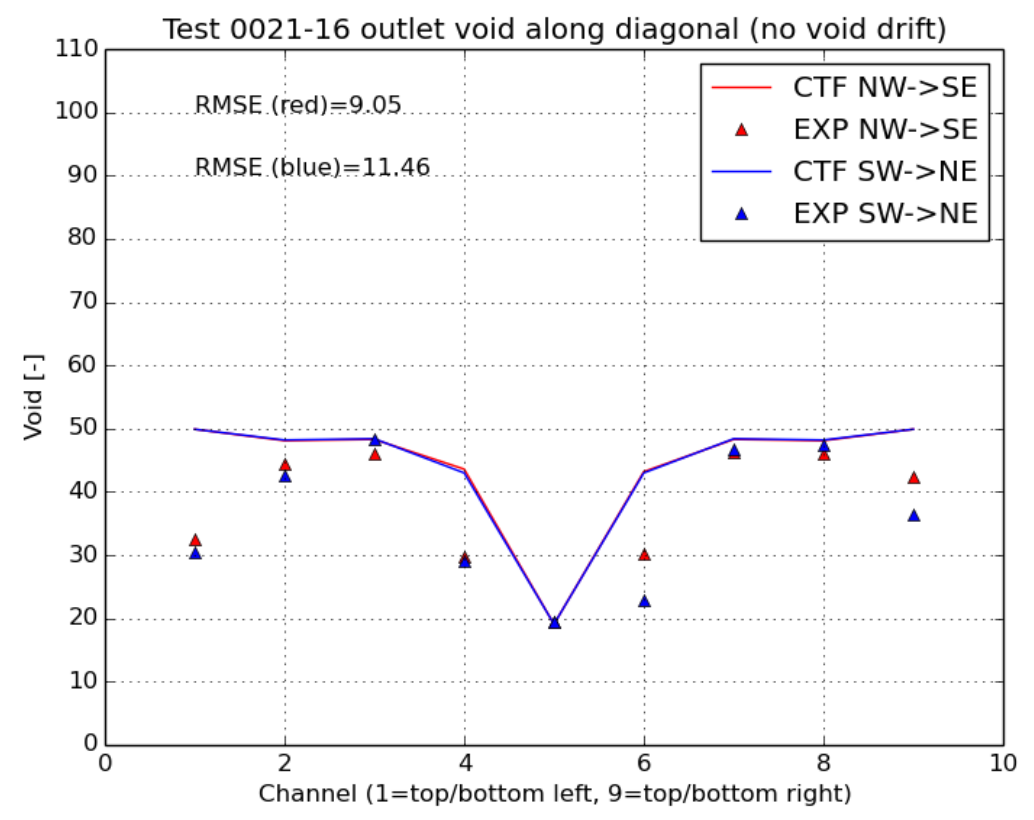

Figure 8.39: Measured and predicted void along diagonals of Test 0021-16 with void drift disabled. 


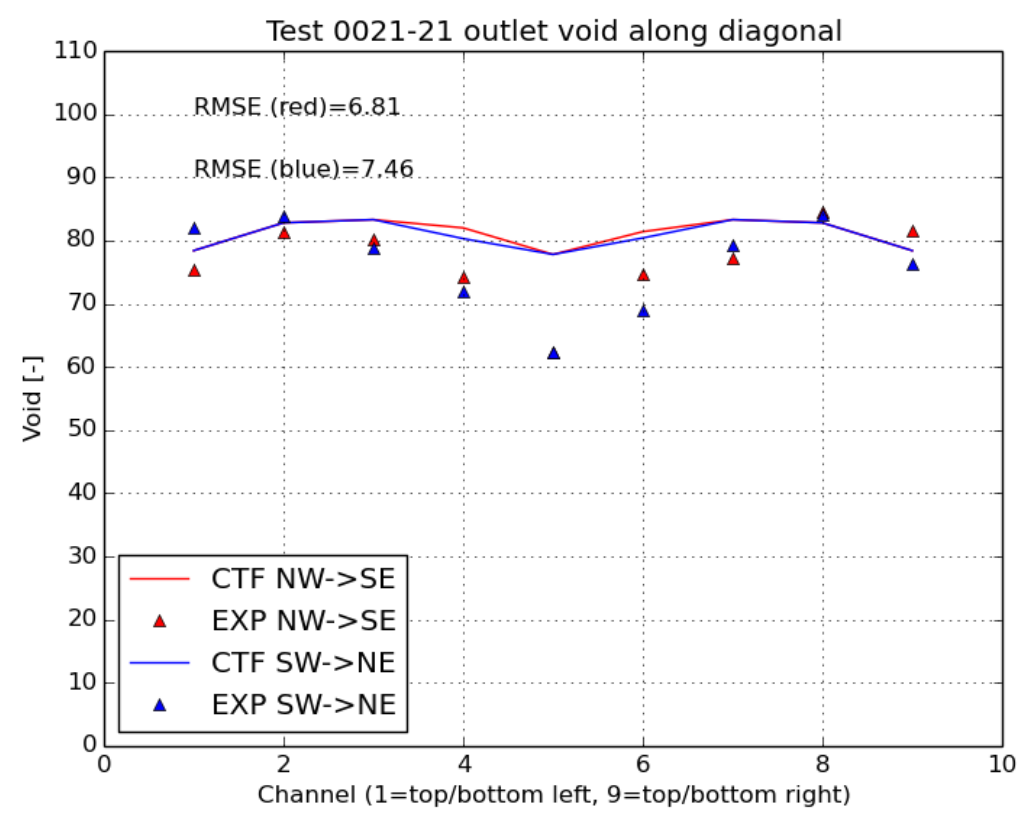

Figure 8.40: Measured and predicted void along diagonals of Test 0021-21.

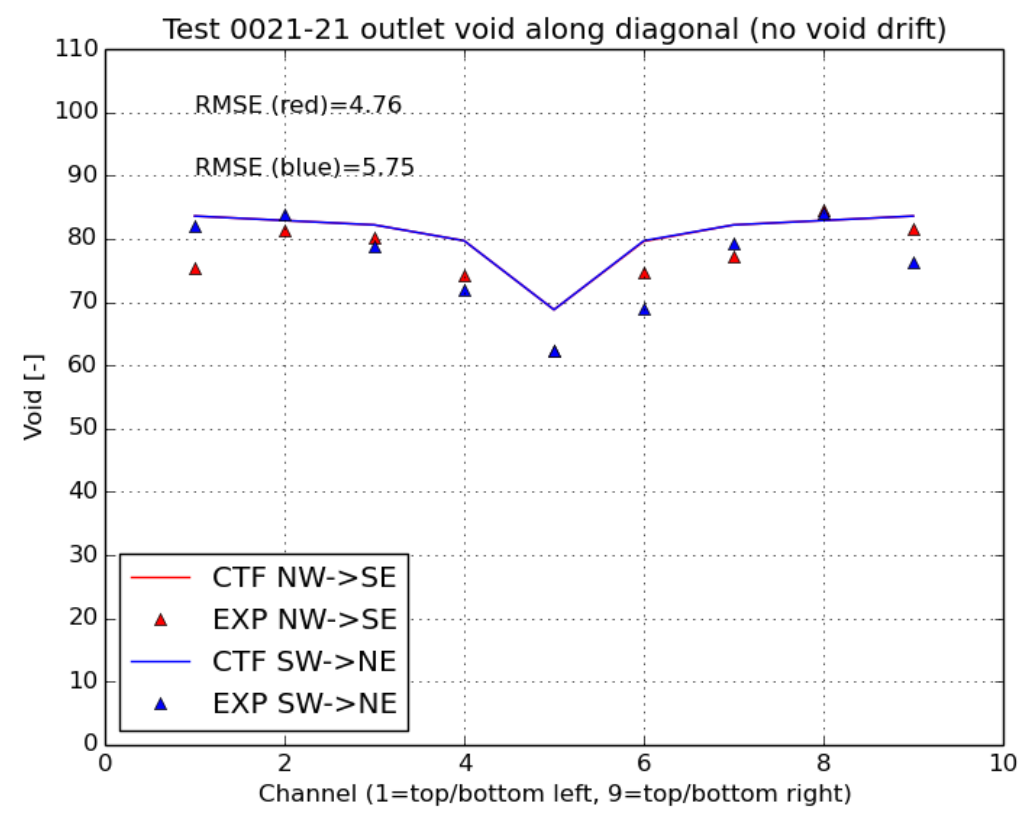

Figure 8.41: Measured and predicted void along diagonals of Test 0021-21 with void drift disabled. 


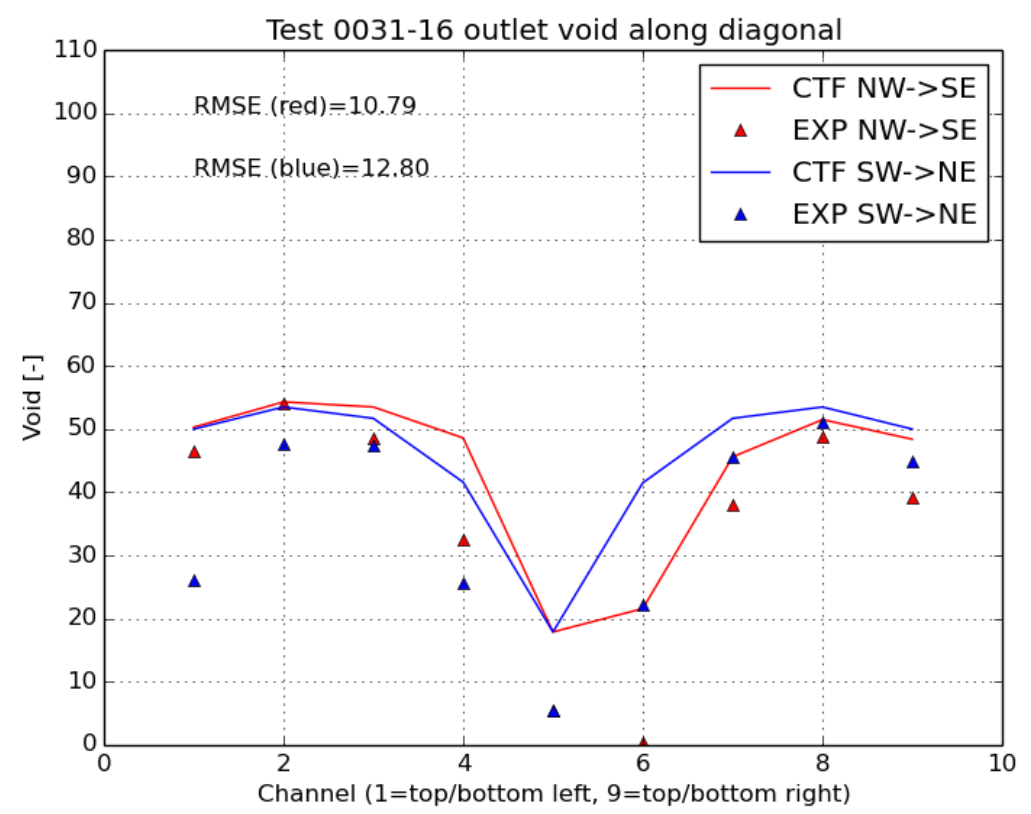

Figure 8.42: Measured and predicted void along diagonals of Test 0031-16.

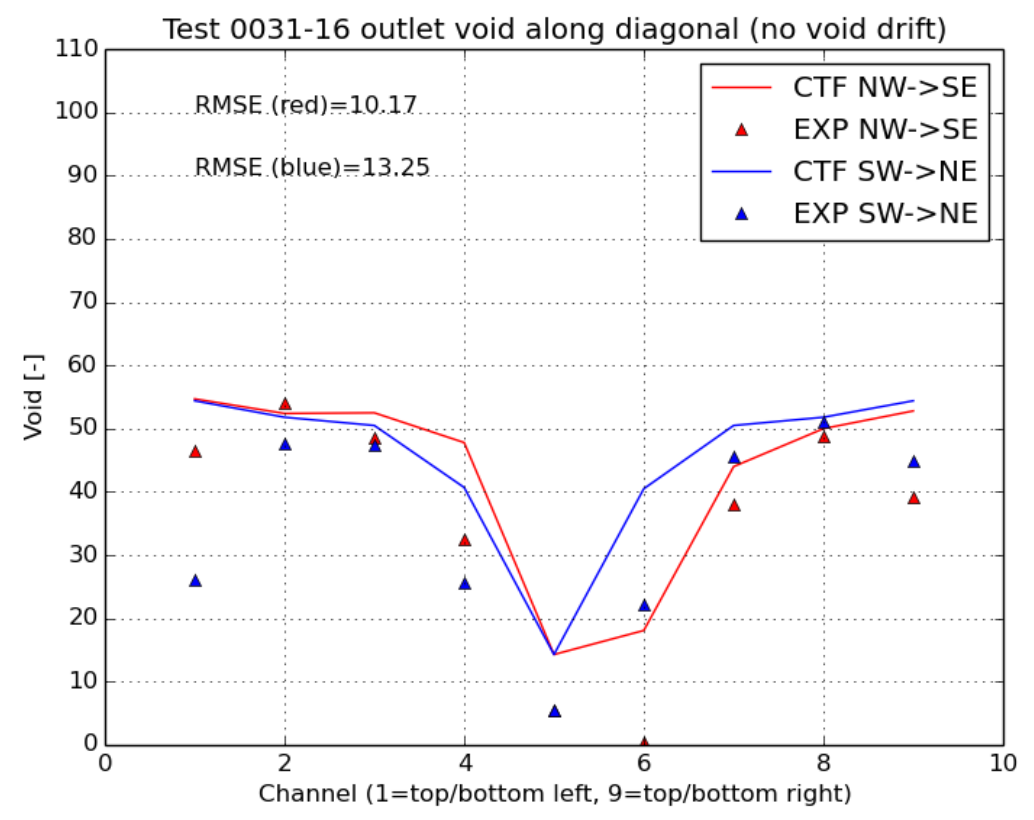

Figure 8.43: Measured and predicted void along diagonals of Test 0031-16 with void drift disabled. 


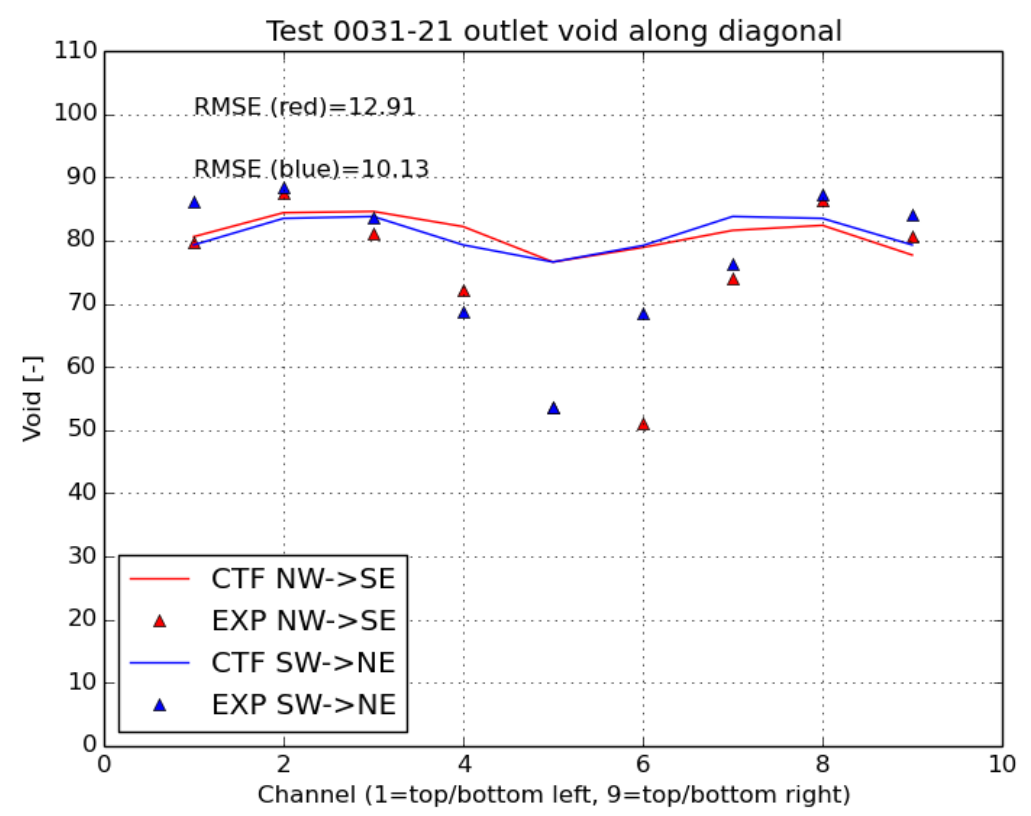

Figure 8.44: Measured and predicted void along diagonals of Test 0031-21.

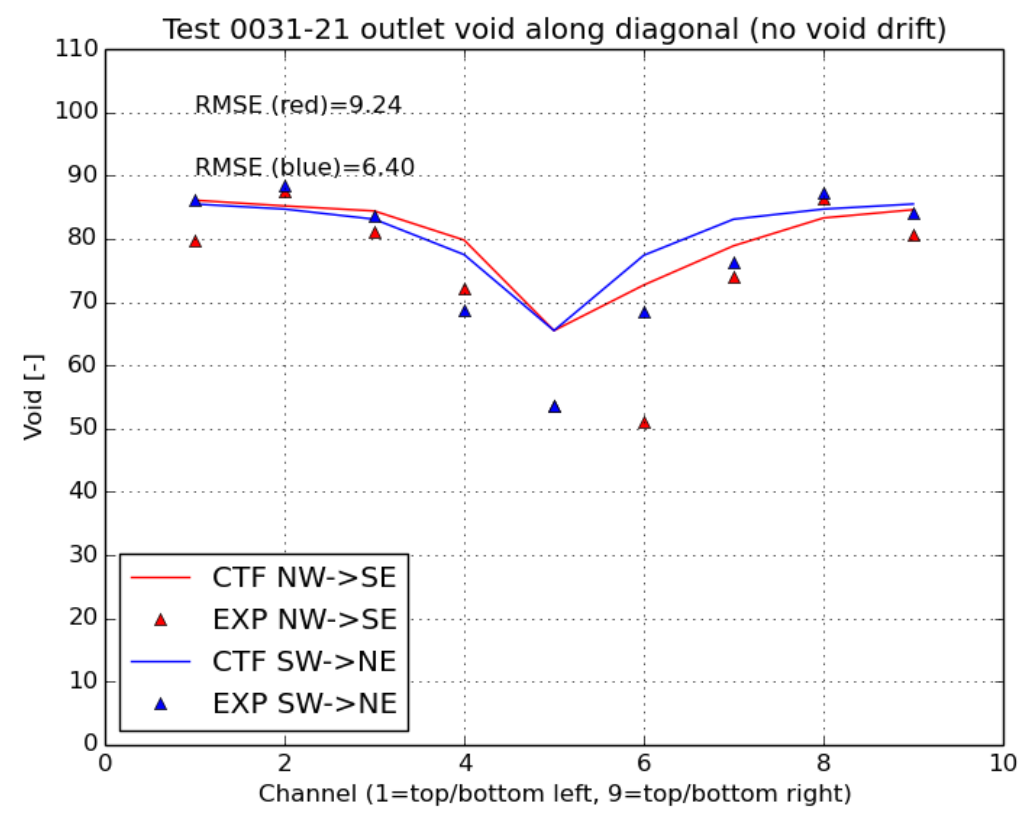

Figure 8.45: Measured and predicted void along diagonals of Test 0031-21 with void drift disabled. 
drift disabled (Figure 8.47). As with other assembly types, the higher-void case comparison is slightly worse with void drift enabled. Figures 8.48 and 8.49 show the results for the high void case with and without void drift, respectively.

Finally, Assembly Type 4 contains a non-uniform radial power distribution, uniform axial power distribution, and one large water rod in the center of the model. Therefore, there is no center channel measurement or CTF prediction for these cases. The low-void case is shown in Figure 8.50 with void drift and Figure 8.51 without void drift. High-void case results are shown in Figure 8.52 with void drift and Figure 8.53 without void drift. Improvements in RMSE are more significant for the lower-void cases, but in general, data compares more favorably for this assembly type than for any of the others that are tested in this study. 


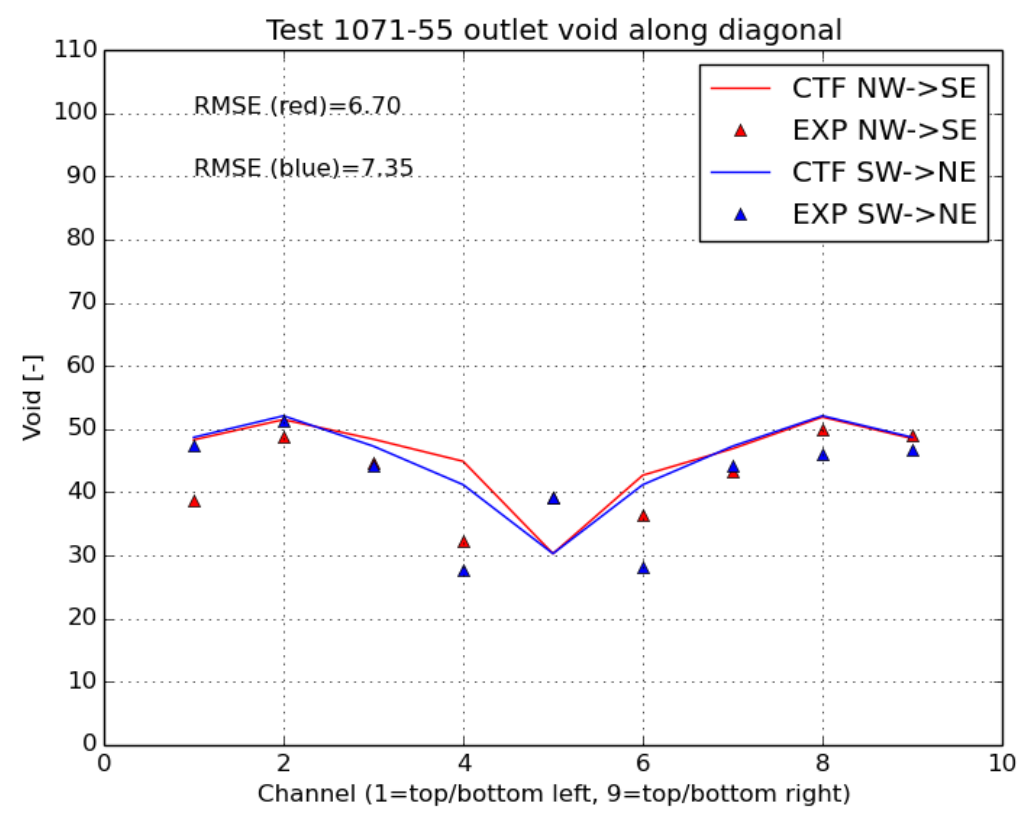

Figure 8.46: Measured and predicted void along diagonals of Test 1071-55.

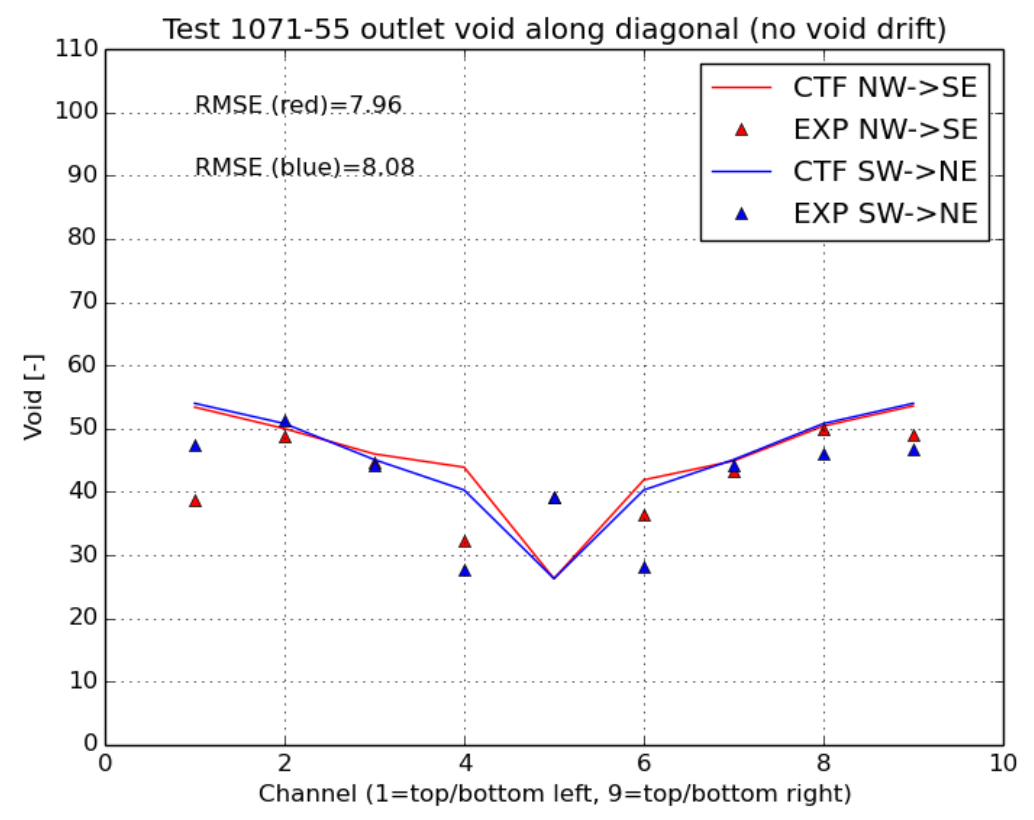

Figure 8.47: Measured and predicted void along diagonals of Test 1071-55 with void drift disabled. 


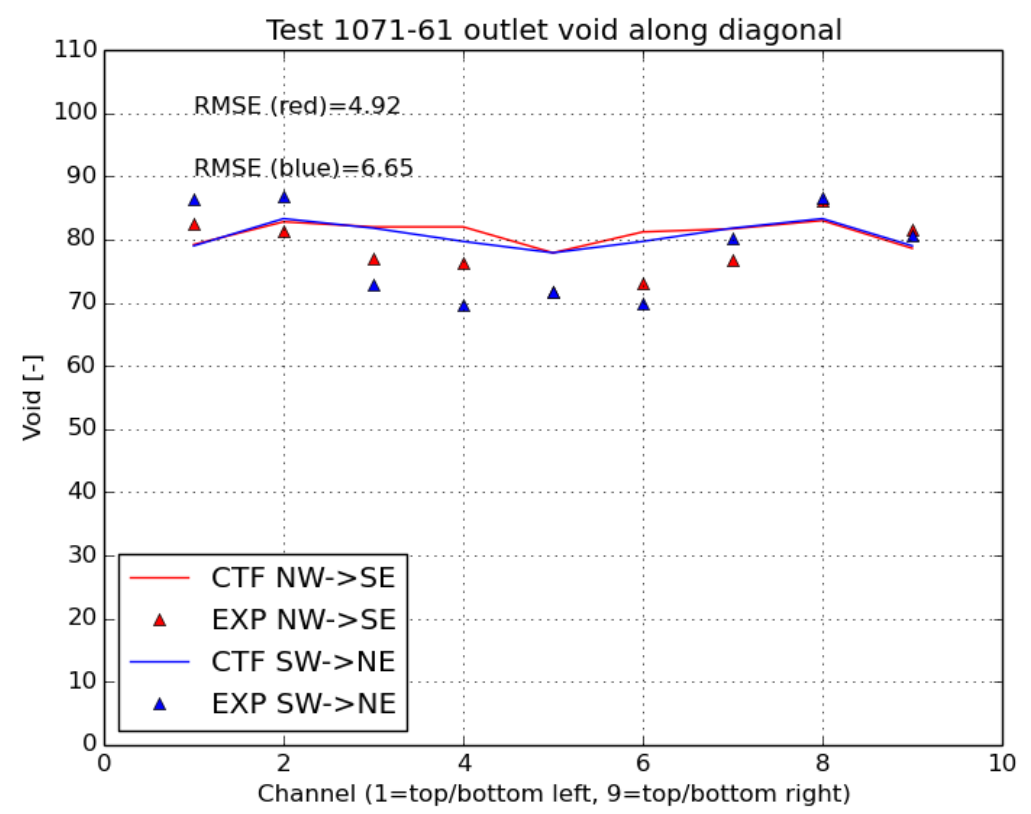

Figure 8.48: Measured and predicted void along diagonals of Test 1071-61.

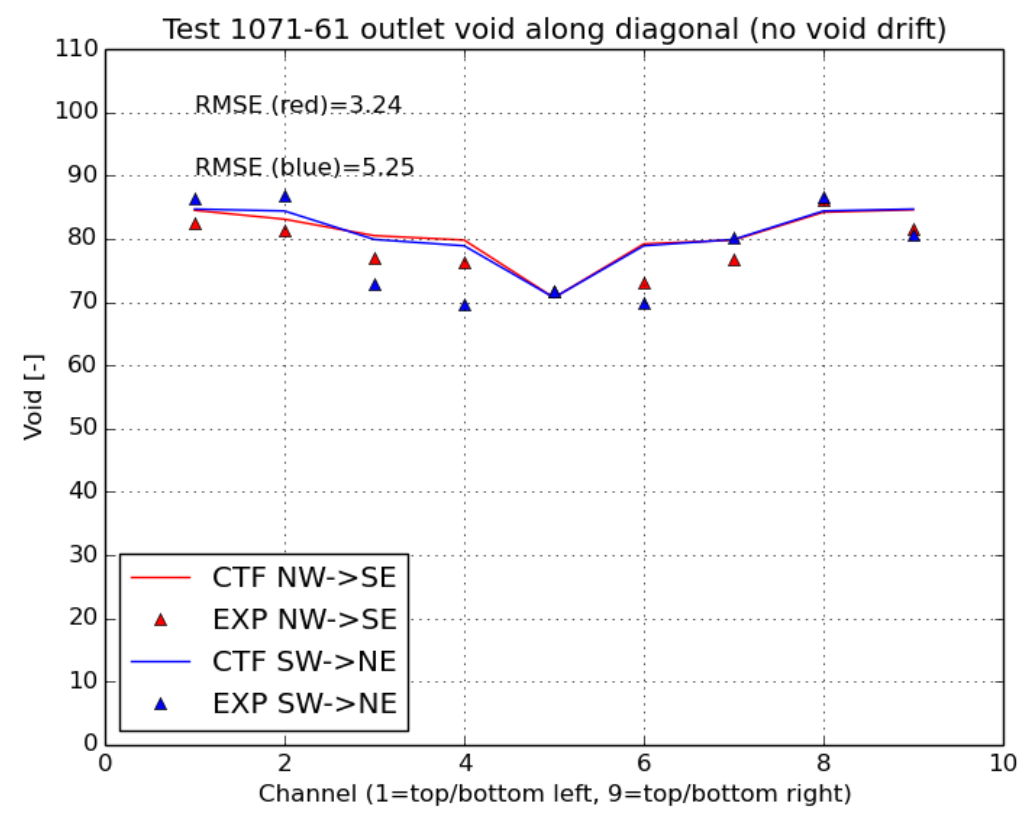

Figure 8.49: Measured and predicted void along diagonals of Test 1071-61 with void drift disabled. 


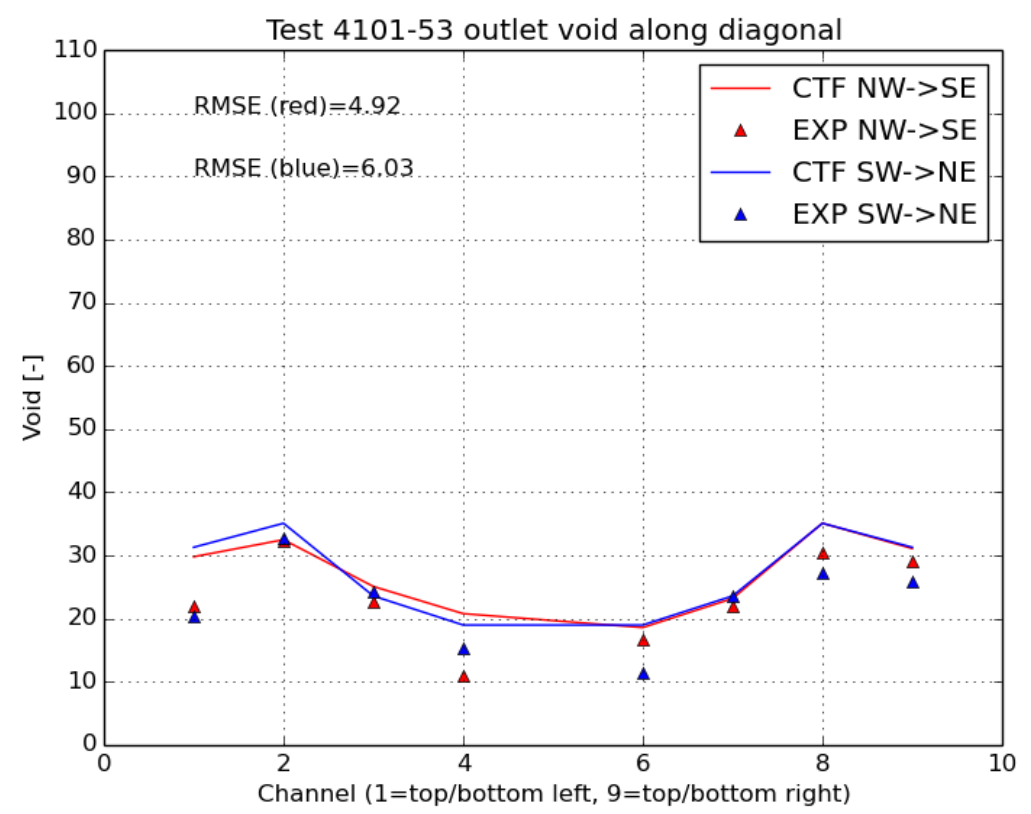

Figure 8.50: Measured and predicted void along diagonals of Test 4101-53.

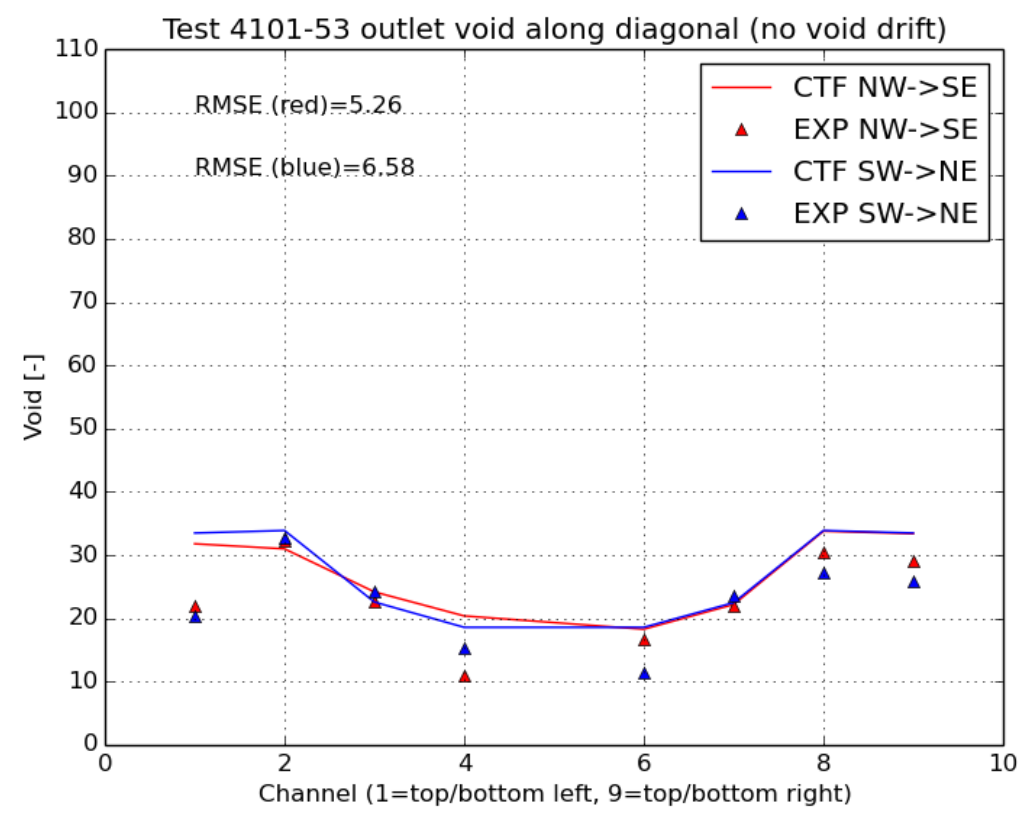

Figure 8.51: Measured and predicted void along diagonals of Test 4101-53 with void drift disabled. 


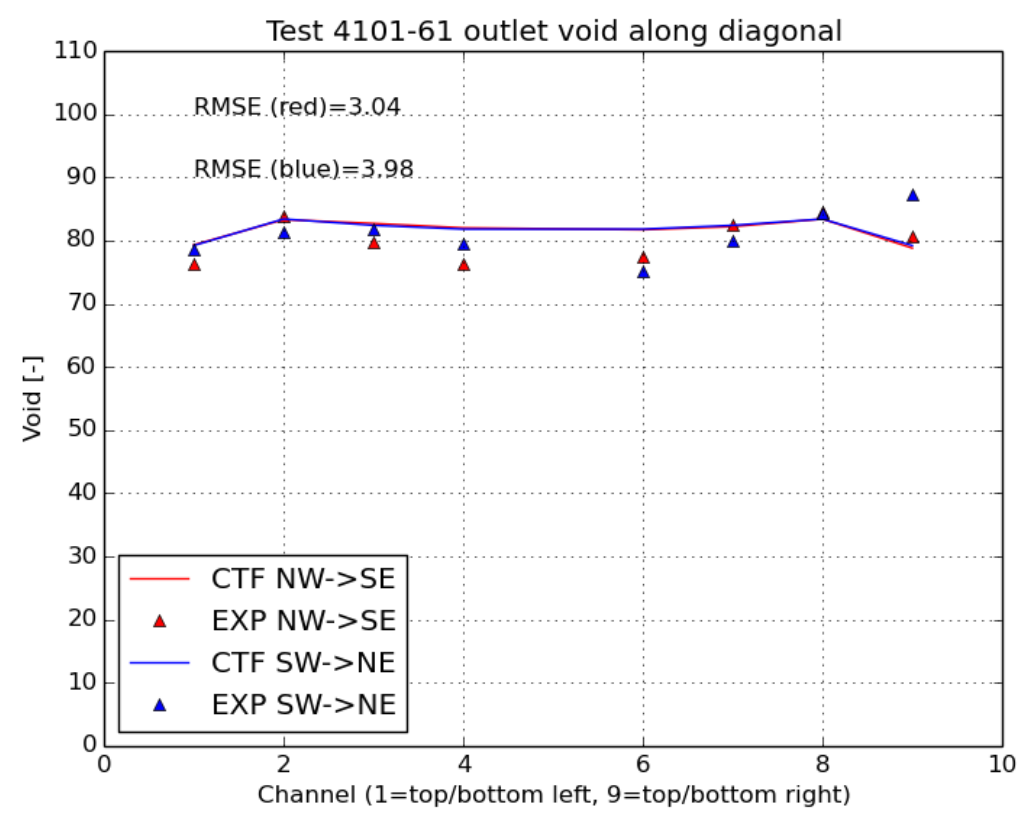

Figure 8.52: Measured and predicted void along diagonals of Test 4101-61.

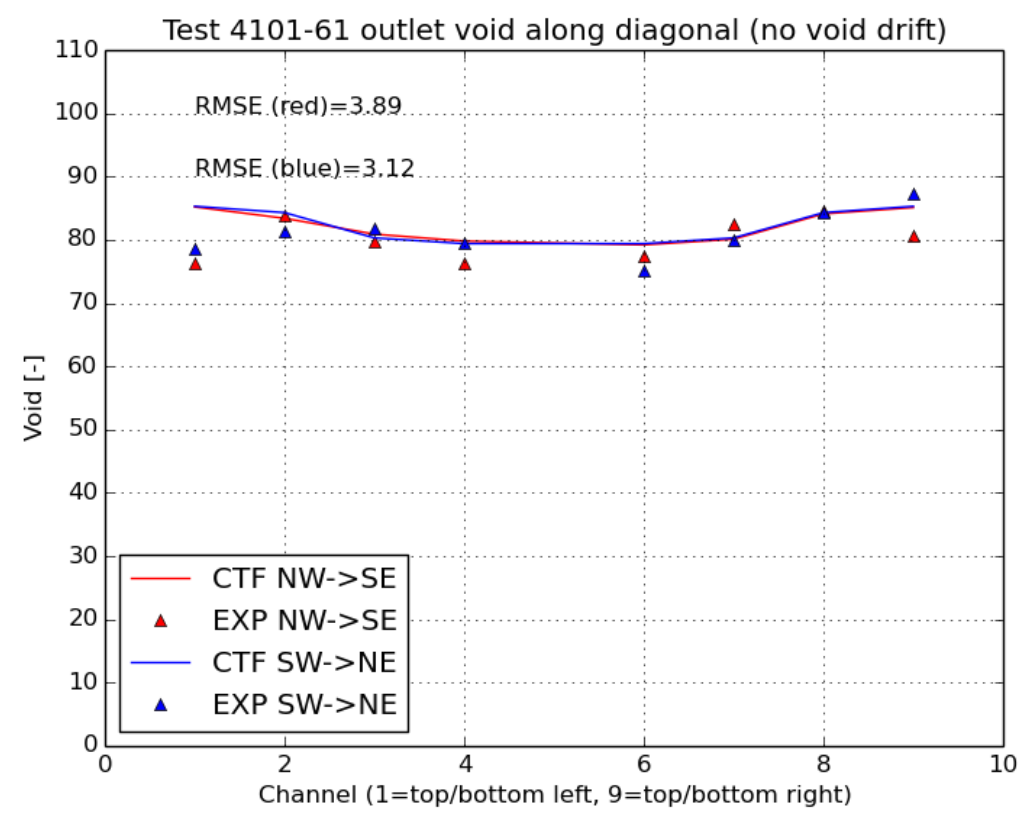

Figure 8.53: Measured and predicted void along diagonals of Test 4101-61 with void drift disabled. 


\section{CHAPTER 9}

DRYOUT

\subsection{Validation}

Prediction of CHF in CTF primarily depends on the CHF model employed which, for these tests, was the "standard" model. This model considers three possible scenarios that can lead to CHF: pool-boiling, forced-convection boiling, and annular-film dryout. The means for selecting which scenario prevails is detailed in Section 4.4.2.1 of the CTF Theory Manual[2]. If in pool-boiling or annular film dryout, a modified-Zuber equation will be used. For forced convection, the Biasi equation is used. The critical heat flux $\left(q_{\text {chf }}^{\prime \prime}\right)$ will be calculated at each timestep during the rod solution. The wall temperature required to make the nucleate boiling heat flux match the critical heat flux is calculated in an iterative fashion; this temperature is known as the CHF temperature $\left(T_{\text {chf }}\right)$. When the actual wall temperature of the rod exceeds this $T_{\text {chf }}$ value, a post-CHF heat transfer regime is entered and DNB occurs.

With this behavior, we can see that the CTF prediction of CHF will be affected by the CHF correlation employed and the heat transfer correlations employed (both single-phase convection and boiling).

\subsubsection{Harwell Facility}

The QOI for these tests is the location where dryout occurs. The experimenters reported the tube surface temperatures recorded by thermocouples attached to the outside of the test tube at discrete axial locations. A steep increase in temperature at a given thermocouple indicates a drop in heat transfer due to vapor blanketing of the tube surface (CHF). This location was selected in a subjective way by choosing the thermocouple reporting a steep temperature increase. Though, as evidenced by Figure 9.1, which shows the axial temperature distribution for one of the CHF tests and CTF predicted values, the point of dryout was typically very obvious. 
Similarly, dryout was determined in CTF by looking at the tube surface temperatures reported in the results output file. A significant increase in temperature indicates a change in the heat transfer regime. Conveniently, the CTF output file also prints the heat transfer regime; a nucleate boiling heat transfer regime is pre- $\mathrm{CHF}$ and a transition boiling heat transfer regime is post-CHF.

A large set of test cases were modeled, 13 in total, in order to cover a wide range of operating conditions and generate good statistics. The predicted dryout location is plotted against the experimental observed dryout location in Figure 9.2. A note about the error bars on the data is in order for this figure. The experimental uncertainty comes from the fact that the thermocouples are placed at discrete locations. If a thermocouple reads an elevated temperature due to vapor blanketing, it does not necessarily mean that CHF occurred at exactly that axial location. Rather, CHF could have occurred at any axial location upstream of that thermocouple, up to the next thermocouple which is not reading an elevated temperature. The experimental uncertainty bars represent this distance to the next upstream thermocouple.

The error bars on the CTF predicted location represent the fact that CTF did not give a single location for CHF. Rather, for some test cases, the tube surface temperature shot up in one axial level, then dropped back down in the next axial level, then shot up in the level above that, and so on. Some test cases gave a single axial location where temperature increased, but others saw two or three oscillations in temperature with increasing height before the temperature became continuously elevated. In the event of such oscillations, the point where temperature became continuously elevated was selected as the predicted dryout location. The error bars on the CTF predictions then wrap any oscillations in temperature that occur upstream of that location. From Figure 9.2, you can see that these oscillation regions were relatively small compared to experimental uncertainty. Furthermore, in no case did the these oscillations result in the CTF predictions falling in the range of experimental dryout location.

The discrepancy between calculated and measured dryout location is capture with the RMSE statistic. As stated before, the CTF dryout location is chosen as the axial location where the wall temperature becomes constantly elevated. The measured dryout location is set at the thermocouple where temperature is observed to rapidly increase, even though the actual dryout location may occur further upstream. Using all 13 data points leads to an RMSE of $0.478 \mathrm{~m}$. From Figure 9.2, we can see that there are two outliers that will skew that statistic significantly. Leaving them out of the calculation leads to a RMSE of $0.281 \mathrm{~m}$.

It is also important to take note of the direction of the skewing, as predicting CHF to occur sooner than it actually does represents a conservative calculation, as CHF is a limiting term in safety analysis. We can see that the two outliers both show that CTF is predicting CHF to occur lower in the test section, or sooner, than it actually occurs. Calculating the average discrepancy for the non-outliers using Equation 9.1, leads to a discrepancy of $-0.129 \mathrm{~m}$. This is also a more conservative prediction, on average, of the $\mathrm{CHF}$ location.

$$
E=\frac{1}{N} \Sigma_{i}\left(x_{\mathrm{ctf}}-x_{\mathrm{exp}}\right)
$$




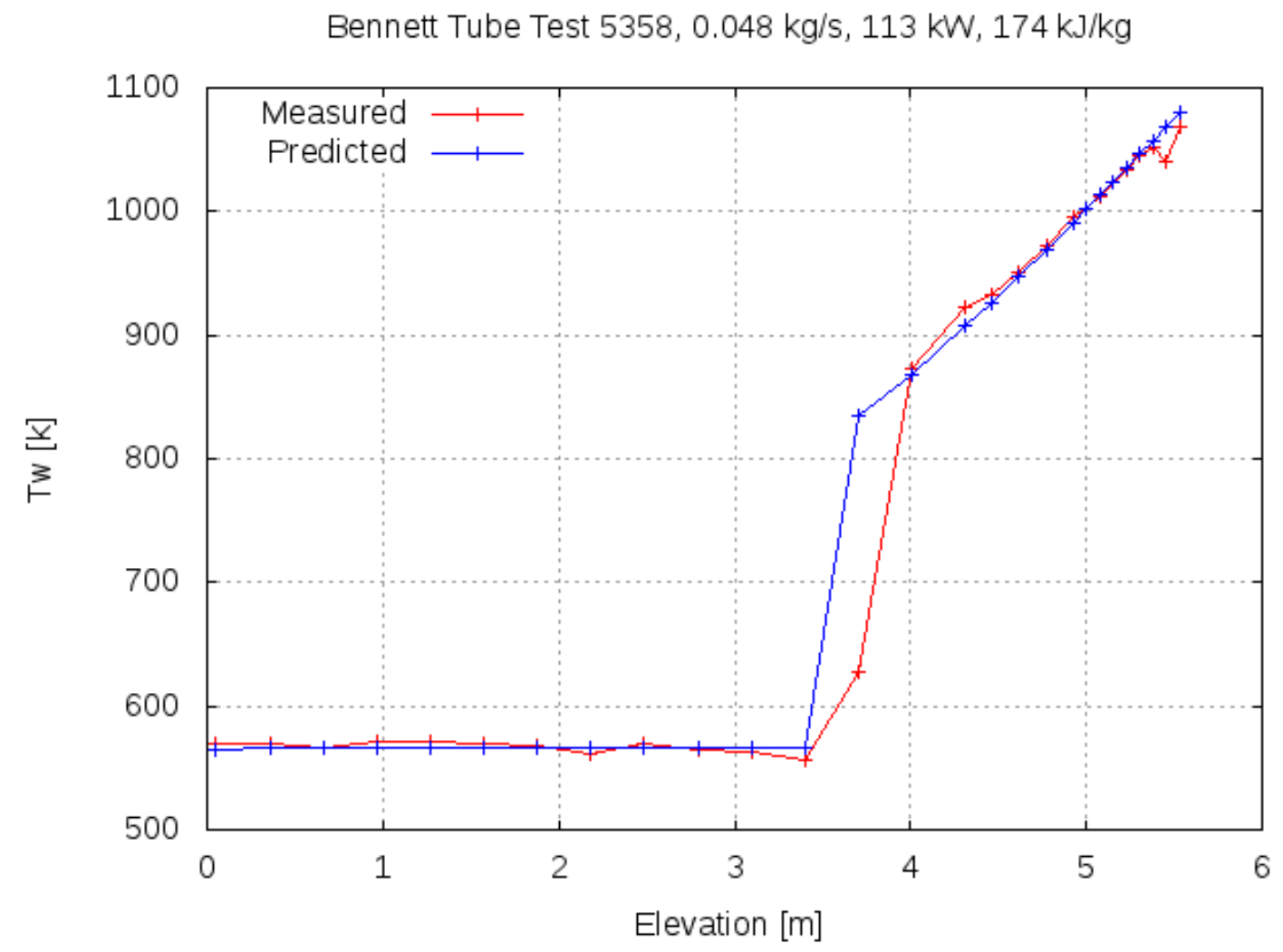

Figure 9.1: Comparison of predicted and experimental axial temperature profiles for Harwell Test 5358 


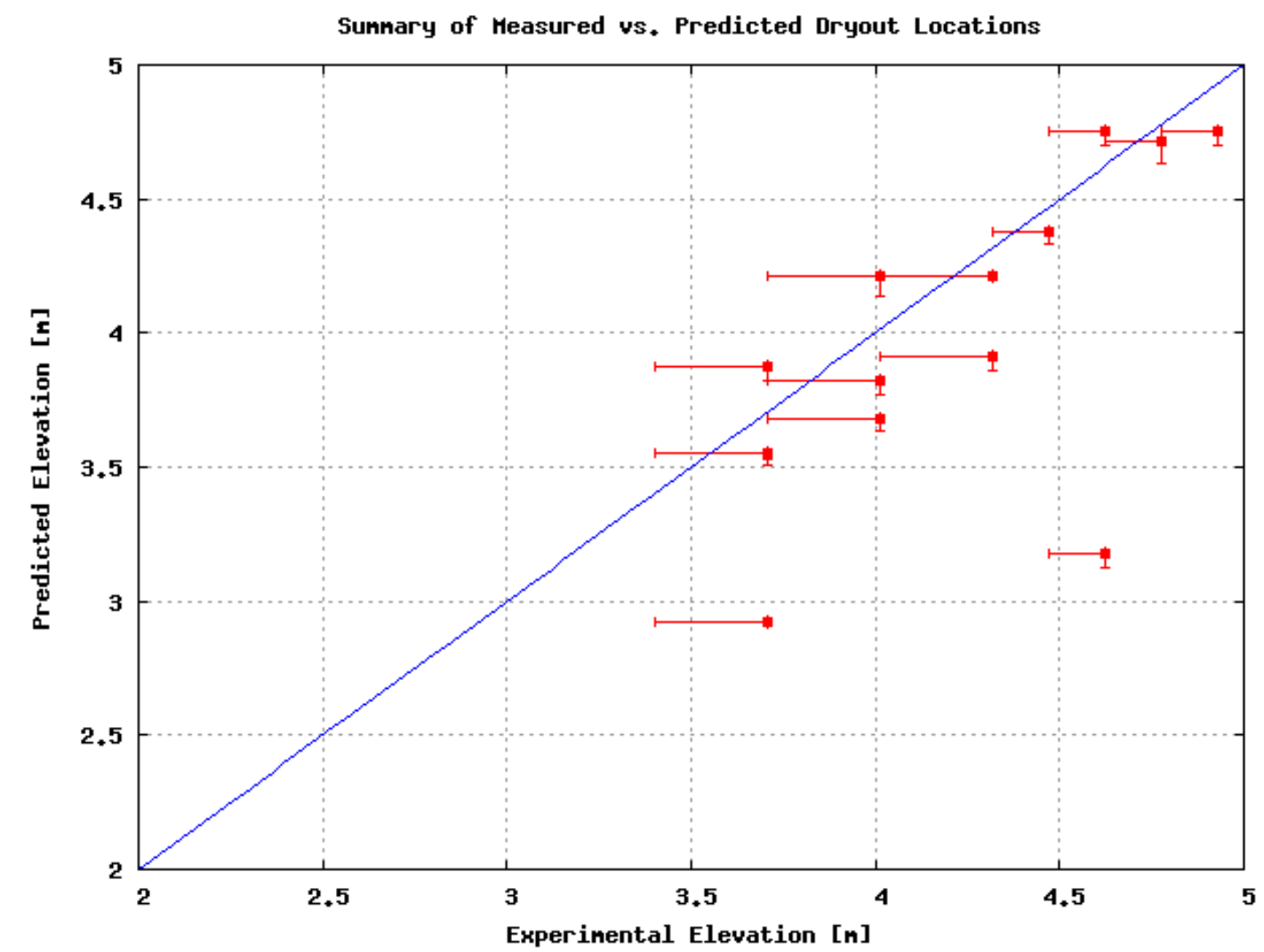

Figure 9.2: Summary of predicted and experimental dryout locations for Harwell test cases modeled by CTF 


\subsubsection{Takahama Tests}

Additional validation of the DNB models of CTF were performed using the Takahama tests[18]. This facility has not been described in Chapter 3 due to its proprietary nature. Additionally, it is not possible to include the specific results of the study in this open document, but mention is made of these tests and the general conclusions are discussed. A more detailed analysis can be found in the technical report, Application of Multi-Scale Thermal-Hydraulic Models to DNB Analysis[18].

In the analysis, CTF was used to model the Japanese Atomic Energy Research Institute (JAERI) sponsored Takahama tests, which simulate the RIA transient. These tests used actual nuclear fuel rod segments having burnups between 40 and 50 GWD/MTU in a specially designed test facility. A short pulse was produced in the rods, representative of the conditions of a RIA. This led to the occurrence of DNB in all tests, with most specimens surviving the transient, but some failing. CTF was able to successfully simulate this fast transient for all modeled test cases. The code correctly predicted the occurrence of DNB compared to experimental observations. Furthermore, the failure mechanism for the test specimens that experience failure was also correctly predicted.

\subsection{Verificaiton}

\subsubsection{Bowring Correlation}

The Bowring correlation [21] is encoded into CTF as an additional option for determining the CHF. This correlation was developed with a wide range of mass-flux and pressure values.

Code Models Assessed Assesses the CHF modeling capability of CTF with the Bowring correlation. This is performed with a unit test designed from a textbook end-of-chapter problem and with a regression test.

Problem Description The unit test portion was taken from Todreas \& Kazimi [22] end-ofchapter problem 13.5 on pg. 818. The input values from the problem are:

- Pressure $=6.89 \mathrm{MPa}$

- Inlet temperature $=204{ }^{\circ} \mathrm{C}$

- Mass flux $=2000 \mathrm{~kg} / \mathrm{m}^{2} \mathrm{~s}$

- Tube diameter $=0.01 \mathrm{~m}$

- Heated length $=3.66 \mathrm{~m}$

- Sat. liquid enthalpy $=1.2617 \times 10^{6} \mathrm{~J} / \mathrm{kg}$

- Sat. vapor enthalpy $=2.7740 \times 10^{6} \mathrm{~J} / \mathrm{kg}$ 
- Inlet enthalpy $=8.7249 \times 10^{6} \mathrm{~J} / \mathrm{kg}$

The enthalpies were determined using the inlet temperature and pressure, and the equilibrium quality and latent heat of vaporization were determined using these enthalpies. According to the problem statement, the result using the Bowring correlation should be $q_{c h f}^{\prime \prime}=1.41 \times 10^{6} \mathrm{~W} / \mathrm{m}^{2}$.

The regression test problem is a $3 \times 3$ model with a central water rod. A cosine shaped axial power shape with a corner peaked radial power profile was used. The model is intended to surpass saturation near the exit, which verifies that the Bowring function does not break under two-phase considerations.

CTF Input Model Description The Bowring single unit test is in Test Group "Bowring CHF Correlation" and is called "Test bowring". It is performed in CTF by passing the state point parameters to the Bowring correlation function. The results are then verified to match the expected values.

The regression test has been added to the automated regression suite as COBRA_TF_run_bowring_verify_3x3 using the model mentioned above.

Discussion of Results The unit test results match the textbook problem.

Conclusions The solution of these tests show that the Bowring correlation is correctly encoded into CTF.

\subsubsection{Groeneveld Look-up Table}

The Groeneveld look-up table [23] of CHF values were developed from curve fits to available experimental data. The CHF look-up tables are encoded into CTF as a $15 \times 21 \times 23$ threedimensional array of CHF values in pressure, mass-flux and equilibrium quality and normalized to $8 \mathrm{~mm}$ tubes. Using the Groeneveld look-up tables required an appropriate interpolation scheme to obtain a single $\mathrm{CHF}$ value at a state point.

The correlation is also accompanied with several form or "K" factors [24]. The definitions of the K-factors have changed over time, but the most recent are being used. Each is applied to the interpolated CHF, and serves to account for additional physical effects in the assembly. Of the eight available $\mathrm{K}$ factors proposed by Groeneveld, three are used in CTF:

- $K_{1}$, Channel geometry factor

- $K_{4}$, Heated length factor

- $K_{5}$, Axial heat flux distribution factor

Code Models Assessed Assesses the Groeneveld CHF interpolation scheme and available K-factors calculations in CTF. 
Problem Description The unit test problem was developed from example problem 13.6 from Todreas \& Kazimi [22] on pg. 802, which is used to verify the calculation of $K_{1}$ and $K_{5}$. The interpolation scheme and $K_{4}$ were verified against a hand calculation. The input to the example problem is as follows:

- Pressure $=15.51 \mathrm{MPa}$

- Mass flux $=3807 \mathrm{~kg} / \mathrm{m}^{2} \mathrm{~s}$

- Sat. liquid density $=594.1 \mathrm{~kg} / \mathrm{m}^{3}$

- Sat. vapor density $=102.1 \mathrm{~kg} / \mathrm{m}^{3}$

- Equilibrium quality $=-0.059$

- Rod diameter $=0.0095 \mathrm{~m}$

- Hydraulic diameter $=0.0118 \mathrm{~m}$

- Heated length $=3.658 \mathrm{~m}$

- Axial location $=1.0 \mathrm{~m}$

- Gap width between rods $=0.0031 \mathrm{~m}$

Note that only $K_{1}$ and $K_{5}$ are used from the example problem for comparison. $K_{4}$ is determined in the example problem using a negative homogeneous equilibrium void fraction, $\alpha_{H E M}$, which is impossible. The version encoded into CTF only calculates $\alpha_{H E M}$ for non-negative equilibrium quality, ensuring that $\alpha_{H E M} \geq 0$ always. $K_{4}$ is then verified by hand calculation.

The hand interpolation of the CHF value begins by reducing the $15 \times 21 \times 23 \mathrm{CHF}$ array to a $2 \times 2 \times 2$ array that bounds the state points. Interpolation is then performed in pressure, mass flux and finally in equilibrium quality to return the interpolated CHF value. Using the pressure, mass-flux and equilibrium quality values, the bounds of this $2 \times 2 \times 2$ array are as follows from the Groeneveld look-up tables:

$$
\begin{aligned}
-0.10 & \leq x_{e} \leq-0.05 \\
14 \mathrm{MPa} & \leq p \leq 16 \mathrm{MPa} \\
3,500 \mathrm{~kg} / \mathrm{s} \cdot \mathrm{m}^{2} & \leq G \leq 4,000 \mathrm{~kg} / \mathrm{s} \cdot \mathrm{m}^{2}
\end{aligned}
$$

The resulting $2 \times 2 \times 2$ array and linear interpolation scheme can be represented in Figure 9.3. The order of interpolation has no impact as it will return the same value. The verification value, $q_{c h f}^{\prime \prime}=0.919 \times 10^{6} \mathrm{BTU} / \mathrm{hr}^{\prime} \mathrm{ft}^{2}$, is the product of the interpolated $\mathrm{CHF}$ from the hand calculation (converted to US units) and the applied K-factors.

The regression test is a $3 \times 3$ model with a central water rod. The axial power profile was cosine shaped with a corner peaked rod. The model is intended to surpass saturation near the exit, which verifies that the Groeneveld function does not break under two-phase considerations. 

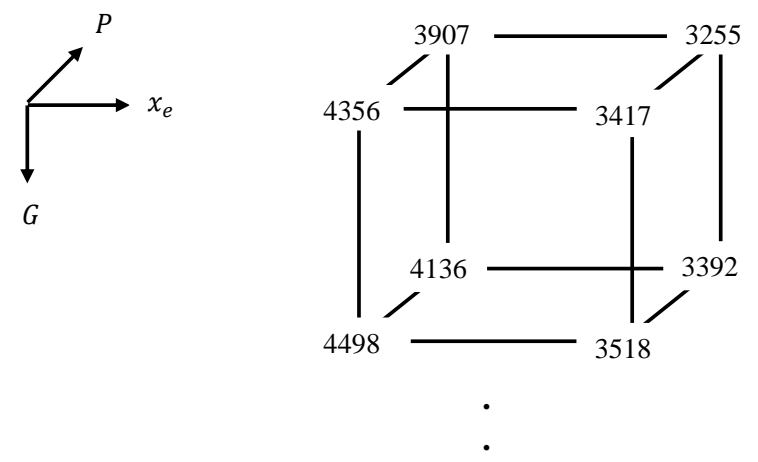

Interpolate in Pressure
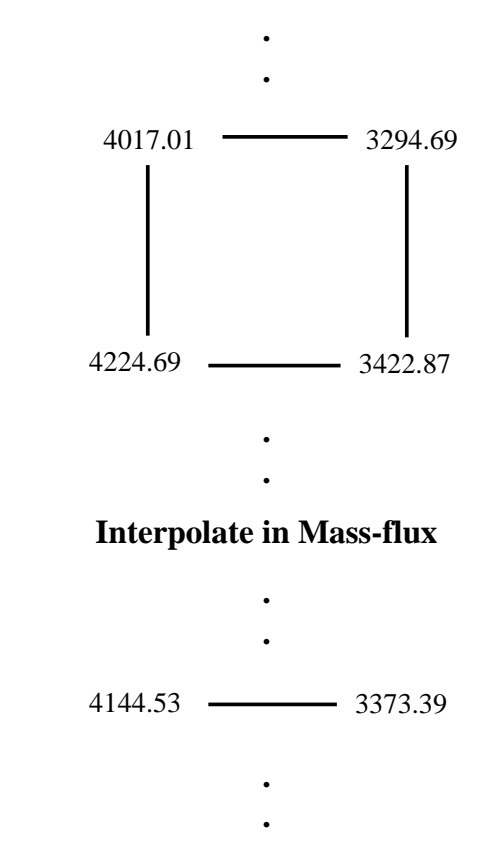

Interpolate in Equilibrium quality

$3512.20 \mathrm{~kW} / \mathrm{m}^{2}$

Figure 9.3: Groeneveld Hand Interpolation Scheme 
CTF Input Model Description The unit test for the Groeneveld correlation is in the "Groeneveld CHF Correlation" test group and is called "Test groeneveld". The test is performed in CTF by passing the state point parameters to the Groeneveld correlation function. The results are then verified to match the expected values.

The regression test has been added to the automated regression suite as COBRA_TF_run_groeneveld_verify_3x3 using the model mentioned above.

Discussion of Results The unit test results match the result of the textbook example.

Conclusions The positive result of the Groeneveld verification test shows that the portion tested is correctly encoded. The example problem used miscalculates $K_{4}$ by using a negative value for $\alpha_{H E M}$. This was user corrected by calculating $K_{4}$ with $\alpha_{H E M}=0$. This test also uses a uniform axial power profile, which initializes $K_{5}$ to unity. An additional unit test should be developed to verify $K_{5}$ for a non-uniform heating. 


\subsection{Inside Tube Flow}

The inside tube flow feature allows CTF to model flow inside solid structures. Experiments with tubular test sections can be accurately modeled by placing the connection to the fluid channel on the inside surface of a heated tube. This feature also allows for multiple channel connections to the inside of a heater rod. An example of where this would be used is the housing of a small rod bundle during a reflood experiment where a quench front would be tracked on the housing using the fine-mesh rezoning model.

Three types of tests were used to verify the correct functionality of this feature:

- Direct Heat Input $(\mathrm{NC}=0)$

- Radial Conduction $(\mathrm{NC}=1)$

- Multiple Inside Channels

All three sets of tests were based on the tube geometry and boundary conditions of the Bennett Tube Test \#5273. However, only the first 20 inches of the test section was modeled using a total of five axial levels. This limited the heat transfer regime to that of subcooled boiling. Also, the very small time steps used in the original Bennett model were increased to make this a fast running problem.

Currently, this feature does not allow for simultaneous fluid connections to the interior and exterior surfaces of a heater rod. This capability will be implemented in a future update. An example of where this capability would be used is for a BWR canister during a LOCA where the fine-mesh rezoning model would be used to track the quench front. 


\subsubsection{Direct Heat Input}

Code Models Assessed Assesses the tube inside flow modeling capability of CTF.

Problem Description The objective was to demonstrate the correct heat input from the rod to an interior fluid channel. The conduction model was turned off by setting the flag $\mathrm{NC}=0$.

CTF Input Model Description Two cases were created: one for inside flow and one for outside flow. The wetted perimeter of the cases was matched so that results were the same between the cases.

The outside flow is called COBRA_TF_run_inflow_1_out. The tube geometry is that of the original Bennett input model with the fluid channel connected to the exterior surface of the tube. The result is used as a base case for other test cases in this series.

The inside flow case is called COBRA_TF_run_inflow_1_in. The tube geometry was modified to match that of the actual experiment, that is, the inside diameter was set to 0.497 in (1.2624 $\mathrm{cm})$ and the outside diameter was set to 0.625 in $(1.5875 \mathrm{~cm})$. The fluid channel connection was set to the interior surface of the tube. All calculated fluid conditions (void fraction, vapor generation rate, etc.) and wall temperatures should be identical to those of the base case (COBRA_TF_run_inflow_1_out).

Discussion of Results Fluid solution results were identical between the two tested cases, as expedted.

Conclusions In this study, the exterior flow capability (channel connected to the outside of a rod) was used to generate a "gold" result file. The insie flow feature was used for the same conditions and was shown to produce identical results to the exterior flow case, thus verifying that the inside flow feature is properly functioning for a single channel in the tube.

\subsubsection{Radial Conduction Test}

Code Models Assessed Assesses the tube inside flow modeling capability of CTF and the radial conduction equations of $\mathrm{CTF}$.

Problem Description Two separate input models were used in this test series as described below. Both tests use the radial conduction model $(\mathrm{NC}=1)$. The purpose was to make sure that the wall heat transfer boundary condition were applied to the correct surface and that the wall temperatures were calculated correctly. The number of radial nodes in the heat structure was increased from 2 to 5 to allow for a more accurate calculation of the temperature drop across the tube wall. 
CTF Input Model Description Two cases were created: one for inside flow and one for outside flow. The wetted perimeter of the cases was matched so that results were the same between the cases.

COBRA_TF_run_inflow_2_in is the name of the model with flow on the inside of the tube. The tube geometry matches that of Bennett experiment with fluid connection on the inside tube surface. Thermal conductivity of the tube wall was set to a constant value of $20(\mathrm{~W} / \mathrm{m}-\mathrm{K}$ to enable comparison to analytical solution. Calculated fluid conditions and inside wall temperature should match those of the base case. The wall temperature drop should be equal to that of the analytical solution for a tube with uniform thermal conductivity and internal heat generation.

COBRA_TF_run_inflow_2_out is the name of the model with flow on the outside of the tube. The connection to the fluid channel was made to the outside surface of the tube as in the base case. The outside tube diameter was set to the ID of the Bennett tube (i.e., 0.497 in [1.2624 cm]). Calculated fluid conditions and outside wall temperature should match those of the base case. The wall thickness, and hence inside diameter, were set so that the temperature drop across the tube wall would match that of the case COBRA_TF_run_inflow_2_in. Specifically, the tube wall thickness was set to $1.49806 \mathrm{~mm}$.

Discussion of Results For the inside flow case, the analytical result is given by:

$$
T_{0}-T_{i}=\left(\frac{q^{\prime}}{4 \pi k}\right)\left[\frac{2 r_{0}^{2}}{\left(r_{0}^{2}-r_{i}^{2}\right)} \ln \left(\frac{r_{0}}{r_{i}}\right)-1\right]
$$

For the conditions of this experiment, namely a linear heat generation rate of $36.494 \mathrm{~kW} / \mathrm{m}$, the temperature difference across the tube wall should be $35.8057^{\circ} \mathrm{C}$. The calculated value was $35.85^{\circ} \mathrm{C}$ and approached the correct value as the number of nodes increased.

For the outside flow case, the analytical solution is given by:

$$
T_{0}-T_{i}=\left(\frac{q^{\prime}}{4 \pi k}\right)\left[1-\frac{2 r_{i}^{2}}{\left(r_{0}^{2}-r_{i}^{2}\right)} \ln \left(\frac{r_{0}}{r_{i}}\right)\right]
$$

For the chosen dimensions, this gives a value of $35.8057^{\circ} \mathrm{C}$. The calculated value was $35.73{ }^{\circ} \mathrm{C}$ and approached the correct value as the number of nodes increased. Figure 10.1 shows the CTF predicted wall temperature drops for increasing levels of radial mesh refinement compared to the analytical solution for both inside flow and outside flow. Both modeling approaches approach the analytical solution as the mesh is refined.

Conclusions This case shows that the inside-flow feature works when radial conduction is enabled in the tube. Additionally, it shows that the conduction equation solution in the tube matches an analytical solution to within $0.1{ }^{\circ} \mathrm{C}$. The solution improves as the mesh is refined. 


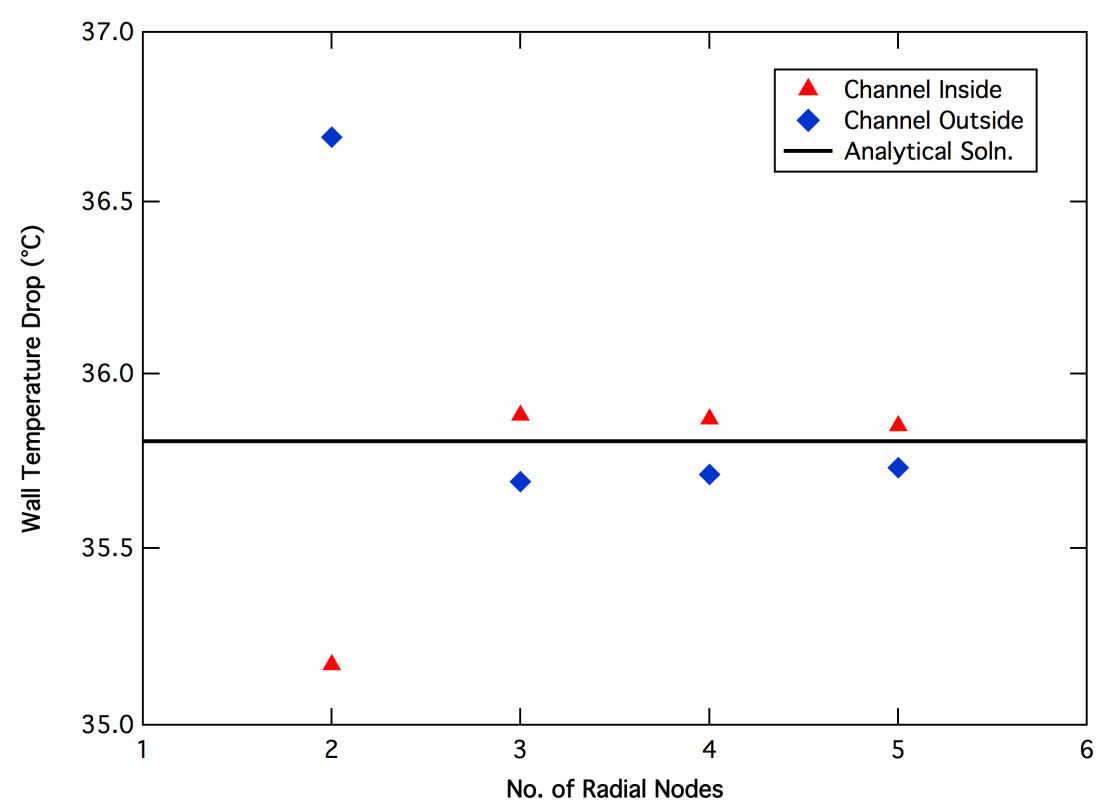

Figure 10.1: CTF predicted wall temperature drop compared to analytical solution for channel on outside versus channel on inside

\subsubsection{Multiple Inside Channels}

Code Models Assessed Assesses the tube inside flow modeling capability of CTF. Particularly, tests the capability to have multiple fluid channels on the inside of a tube instead of only one.

Problem Description The input model of COBRA_TF_run_inflow_2_in was modified for this test and is labeled COBRA_TF_run_inflow_3. Specifically, the original one fluid channel on the inside of the tube was replaced by two channels. Each of these half-channels had half the flow area and wetted perimeter of the original one channel. Likewise, they connected to half of the heater perimeter of the tube inside wall. No gaps were provided between these two channels so they were simply 1-D pipes connected to half of the tube wall.

Both half-channels should have identical results and be equal to the calculated fluid parameters of the base case with two exceptions. That exception is that the flow rates and vapor generation rates should be exactly one-half of those in the base case. The wall temperature solution should match that of the COBRA_TF_run_inflow_2_in case.

\section{CTF Input Model Description}


Discussion of Results The wall temperature solution matched the COBRA_TF_run_inflow_2 in case. The flow rates and vapor generation rates in the inside channels were half that of the COBRA_TF_run_inflow_2_in case, as expected.

Conclusions This verification study showed that CTF is capable of modeling multiple channels inside of a tube. Furthermore, the fluid results match the base case, COBRA_TF_run_inflow_2_in. 


\section{CHAPTER 11}

\section{NATURAL CIRCULATION}

Natural circulation does not classify as an "effect" like the previous sections; it is really a state of operation that has its own effects. However, because it may be of special interest to the user to see how CTF performs when modeling buoyancy-driven natural-circulation flows, it has been given its own section. The primary test of interest here is the PNNL $2 x 6$ case, though the reader should be aware that the FRIGG facility, discussed in Sections 5.3.1.2 and 7.2 was also operated at natural circulation conditions.

\subsection{PNNL 2x6 Tests}

The facility was operated in two different scenarios; steady state and transient conditions. The steady-state results are discussed here. Two types of measurements were made in the facility: LDA local-velocity measurements and subchannel-center thermocouple measurements. Both of these measurement types were taken at five discrete axial locations in the bundle. The LDA measurements were taken along a single 1-D line, or "rake", in each of the three rows of subchannels. The thermocouple measurements were taken along a single 1-D rake in one of the rows of subchannels (the center row). The main difference between the two measurement types was that the LDA measurements were taken in finer increments, allowing for a mapping of the velocity profile within a subchannel, whereas the thermocouple measurements were only taken at subchannel centers.

The experimental results of these tests do not lend themselves as well to quantitative comparison with CTF predictions as well as for other effects that were discussed. This has to do with the fundamental differences between what the measurements and predictions mean. Concerning the velocity measurements, the values were local, so a velocity measurement taken at the center of the subchannel is much higher than that taken in the gap region between rods, where wall drag and viscous effects have a much larger impact on the fluid, leading to slower flows. While this appears in the measurements, the CTF prediction is one averaged velocity that includes 
these wall effects and smears them across the entire cross section of the subchannel.

One workaround to this problem is to average the LDA measurements, but this requires that the measurements be taken at equal intervals, so as not to weight some measurements more than others. This was not the case during the experiments. Additionally, the actual velocity values were not made available in the report but, rather, values were extracted from figures using a digitizer. A second option is to fit a correlation to each subchannel velocity profile and then integrate that curve over the bounds of the subchannel. Even this approach wouldn't be entirely accurate, however, as the subchannel dimensions where the LDA rake was made does not represent the geometry of the channel if we were to move the rake line closer or farther from us, the observer. In lieu of these considerations, the figures showing predicted and measured values are simply presented here for each window and rake location. The LDA results are presented in Figures 11.1 through 11.11 .

In these figures, the LDA location in the rod bundle is shown on the $x$-axis. The $y$-axis of the figure shows a non-dimensionalized velocity at that measurement location (see Figure 3.14 for the meaning of the $\mathrm{X}$ and $\mathrm{Y}$ dimensions of the test assembly). Note in the results figures how the measured velocities will move above and below CTF values in many cases, which is expected, as the CTF values are a smeared, averaged value for the entire channel. The important thing to look for in these figures is that CTF is capturing the behavior of the velocity distribution, which should be for "left-side" velocities to be higher than "right-side" velocities due to the fact that only the "left-side" rods are heated. This uneven heating creates thermal plumes that drive the flow upwards on the left-side of the bundle.

CTF generally captures this behavior for all axial levels for rake locations at $\mathrm{Y}=0.0$ inch and $\mathrm{Y}=0.581$ inch. CTF tends to over-predict velocities for the $\mathrm{Y}=-0.581$ inch rake location. However, the fact that measurements at rake location $Y=-0.581$ inch disagree with those at rake location $\mathrm{Y}=0.581 \mathrm{inch}$ is a cause for concern because the measurements should agree due to symmetry of the test conditions. It was noted by the authors that there was a misalignment of the flow housing which accounts for this discrepancy. Since we do not capture this effect in CTF, we can disregard the disagreement of measured and predicted results for the $\mathrm{Y}=-0.581$ inch rake location.

Figures 11.12 through 11.16 provide the comparison of measured and predicted temperature results for the 9 axial locations. These results will naturally suffer from the same problem as the velocity results. Here we are comparing a measurement of temperature taken at the center of the subchannel with a predicted value that is a smeared average over the whole subchannel. Being that most of these axial measurement locations are taken in a laminar flow, we expect very steep gradients in temperature as we move away from the wall. This accounts for the fact why we see an over-prediction of temperatures by CTF in many cases. However, note how this over-prediction only occurs in the lower axial regions of the bundle. When we move to the higher windows (7 and 9), the results match quite well. The reason for this is likely that the thermal plumes surrounding the rods have merged and led to a turbulent flow in the upper section of the bundle. The increased turbulence acts to mix the fluid and reduce the temperature gradient across the subchannel. Data in this turbulent region will, naturally, match the volume-average results of CTF better.

Again, we need to concern ourselves with CTF's capability to match the behavior of the data. This is mostly the case except for the far-left side of the plots, where we see a drastic drop in measured temperatures. Since the entire left side of the bundle is heated, this drop 


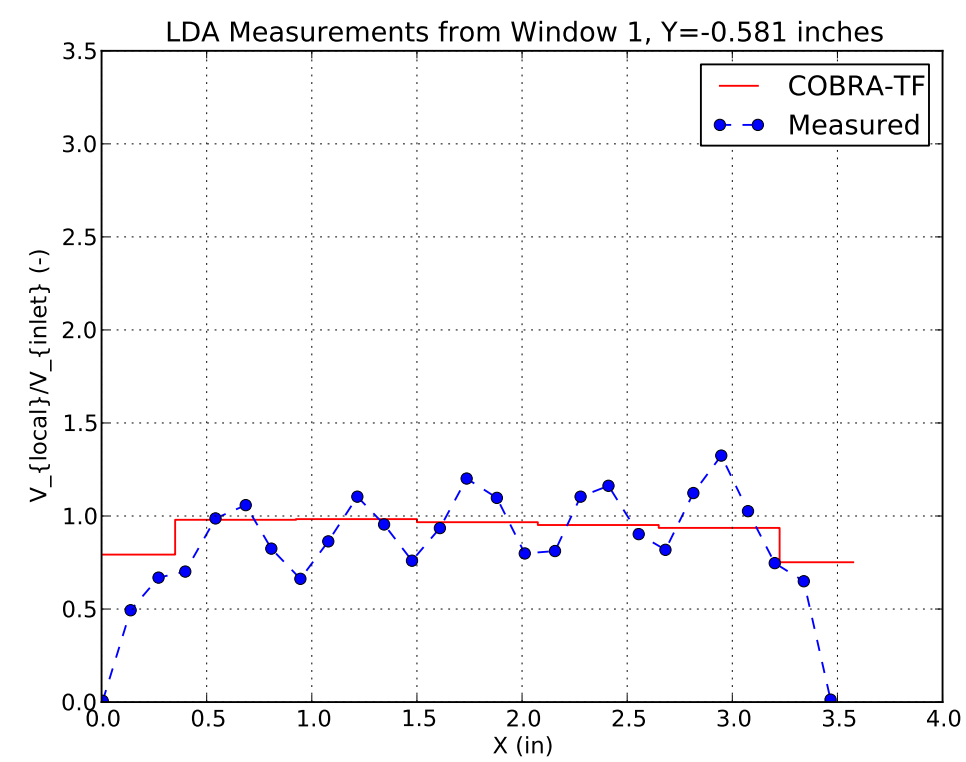

Figure 11.1: Predicted and measured subchannel velocities for Window 1 at Rake Location $\mathrm{Y}=-0.581$ in in PNNL 2x6

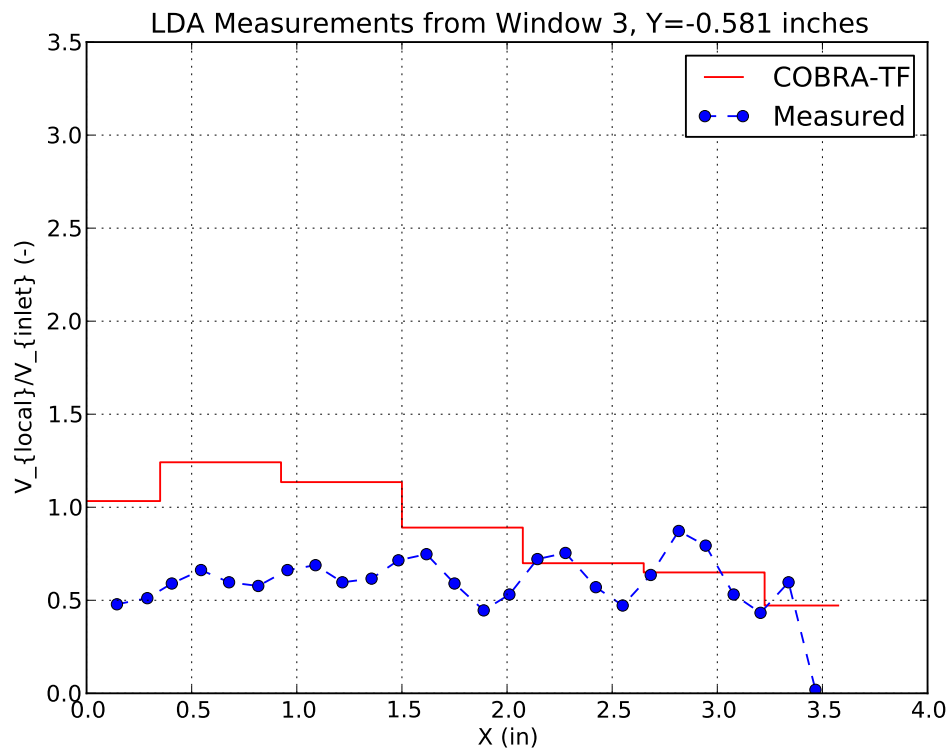

Figure 11.2: Predicted and measured subchannel velocities for Window 3 at Rake Location $\mathrm{Y}=-0.581$ in in PNNL 2x6 


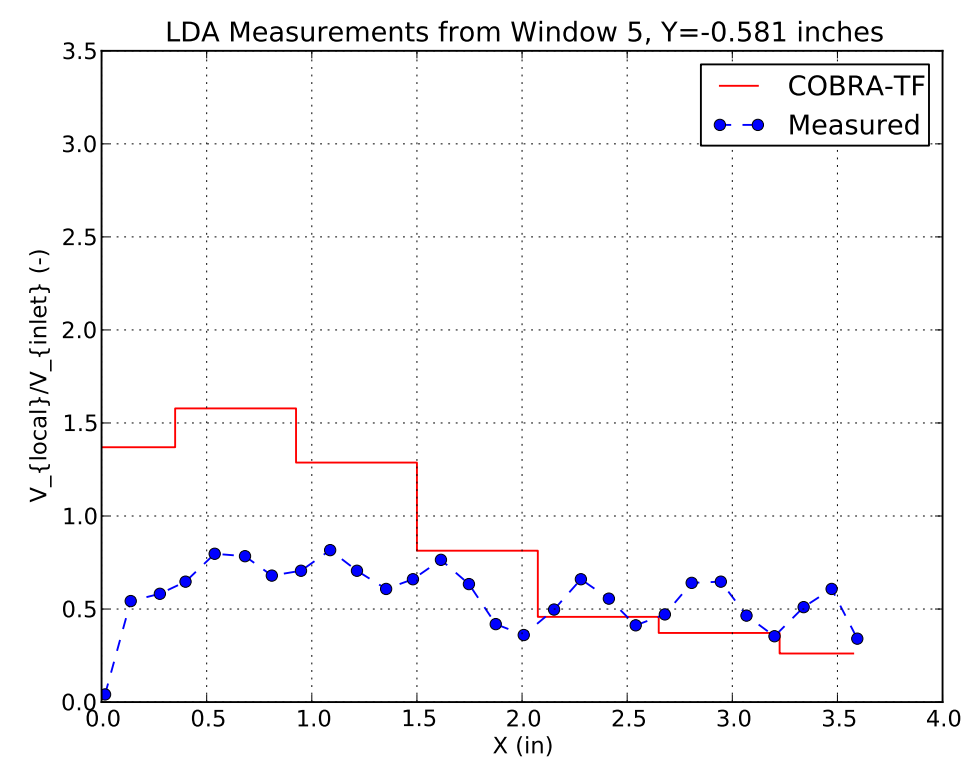

Figure 11.3: Predicted and measured subchannel velocities for Window 5 at Rake Location $\mathrm{Y}=-0.581$ in in PNNL 2x6

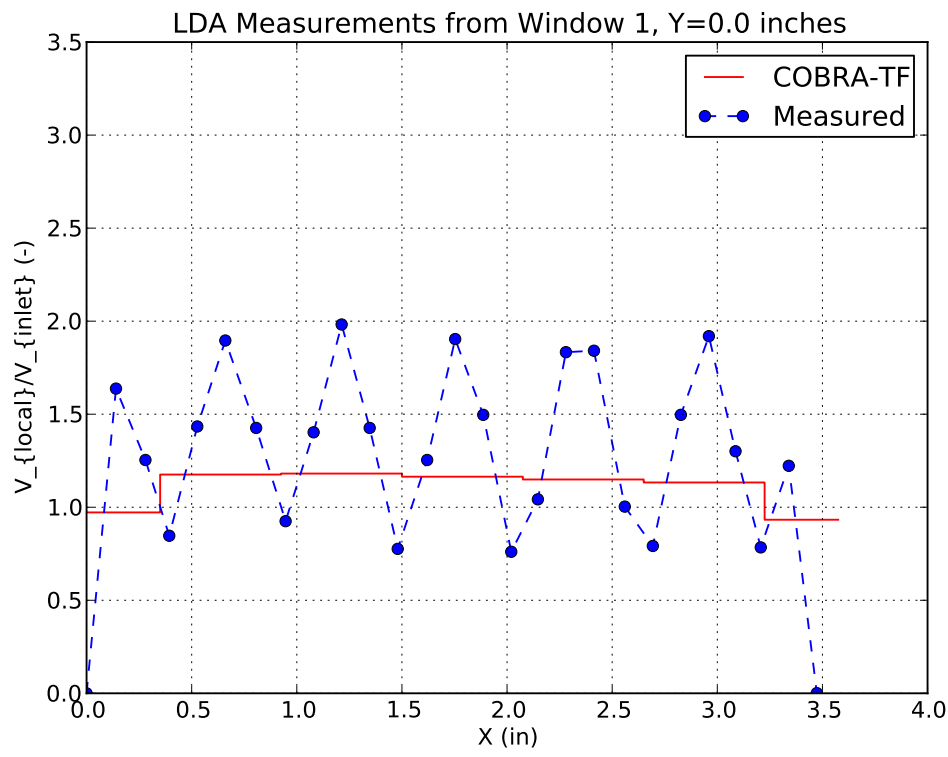

Figure 11.4: Predicted and measured subchannel velocities for Window 1 at Rake Location $\mathrm{Y}=0.0$ in in PNNL 2x6 


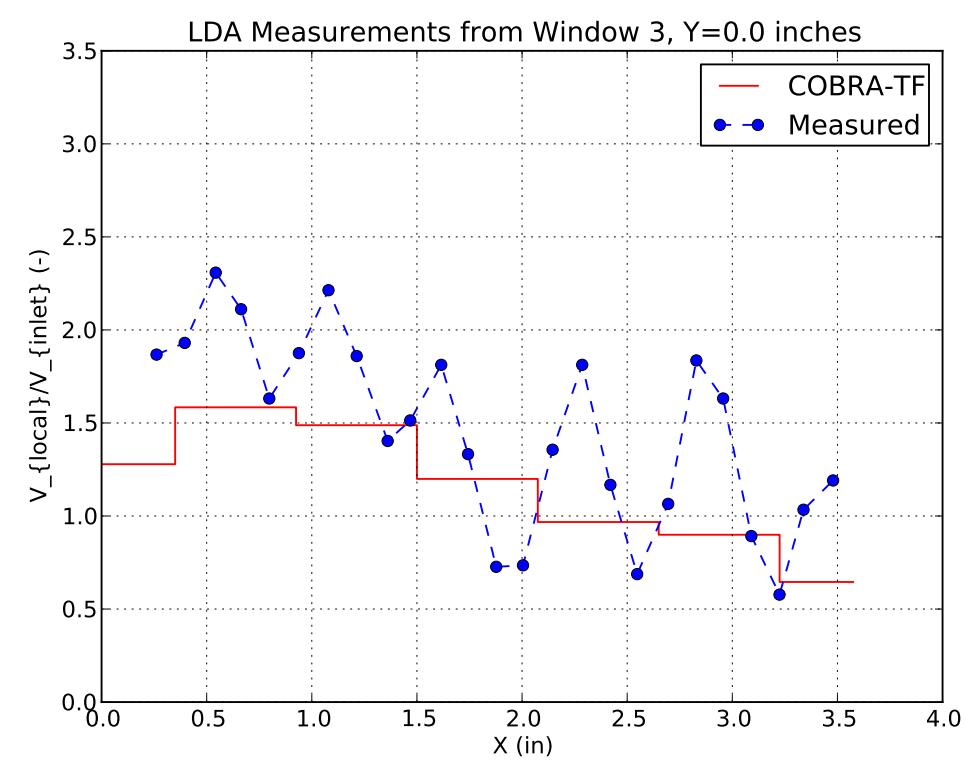

Figure 11.5: Predicted and measured subchannel velocities for Window 3 at Rake Location $\mathrm{Y}=0.0$ in in PNNL 2x6

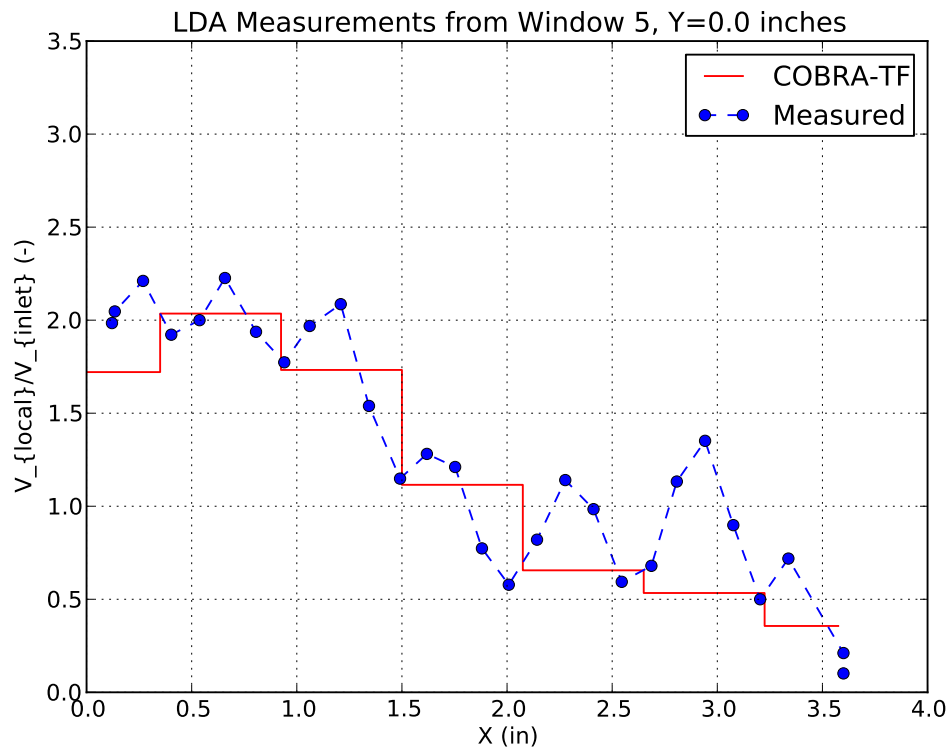

Figure 11.6: Predicted and measured subchannel velocities for Window 5 at Rake Location $\mathrm{Y}=0.0$ in in PNNL 2x6 


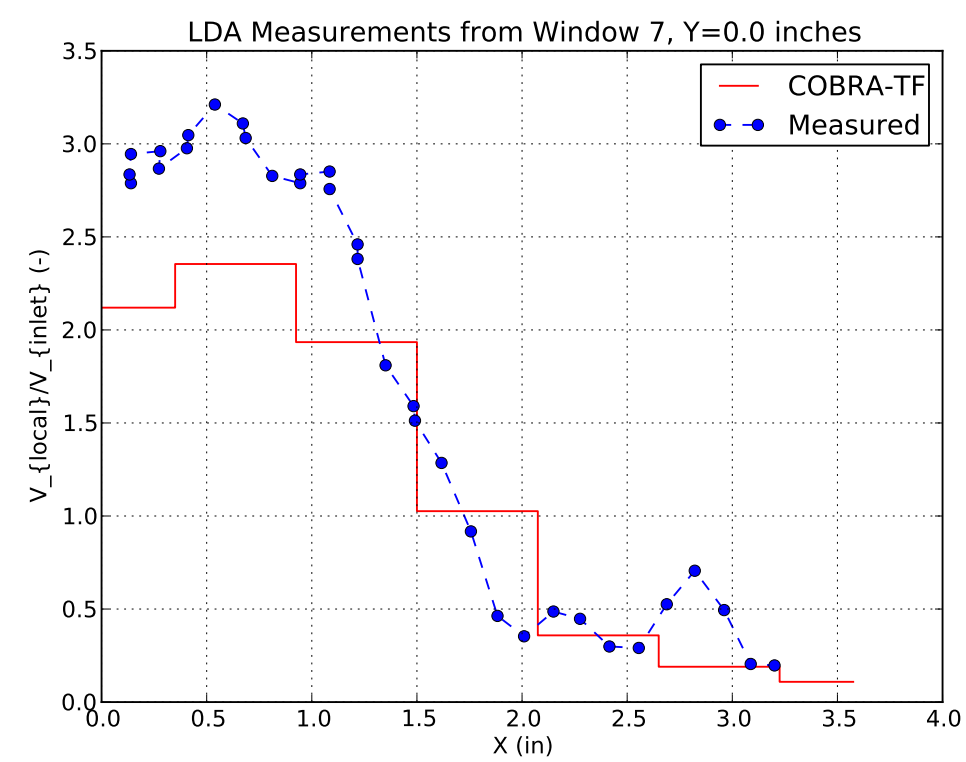

Figure 11.7: Predicted and measured subchannel velocities for Window 7 at Rake Location $\mathrm{Y}=0.0$ in in PNNL 2x6

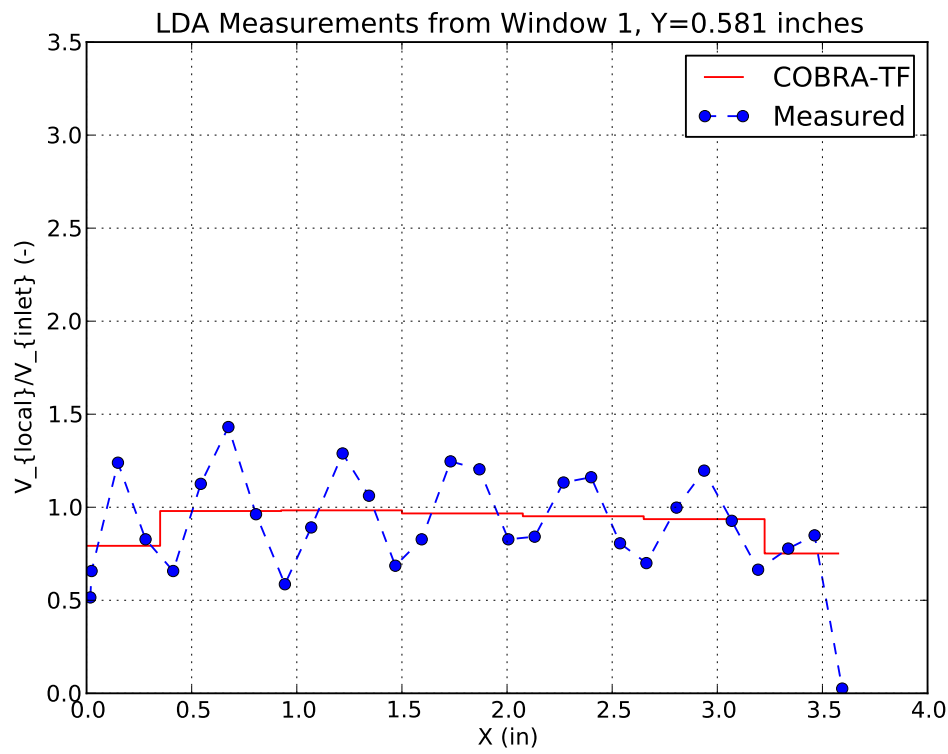

Figure 11.8: Predicted and measured subchannel velocities for Window 1 at Rake Location $\mathrm{Y}=0.581$ in in PNNL 2x6 


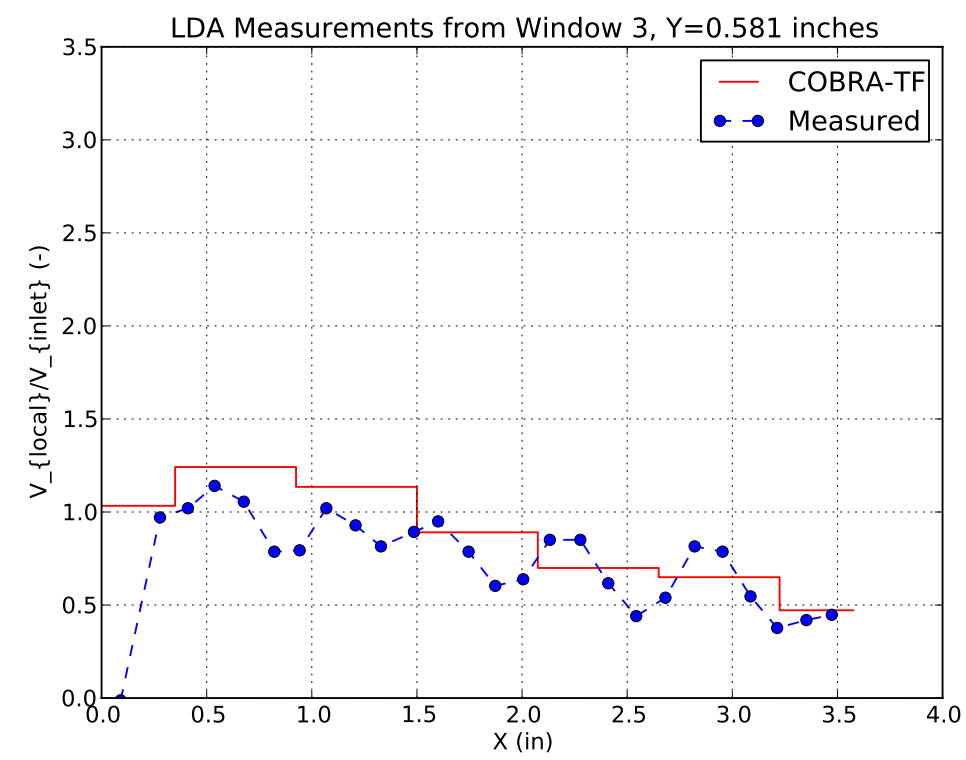

Figure 11.9: Predicted and measured subchannel velocities for Window 3 at Rake Location $\mathrm{Y}=0.581$ in in PNNL 2x6

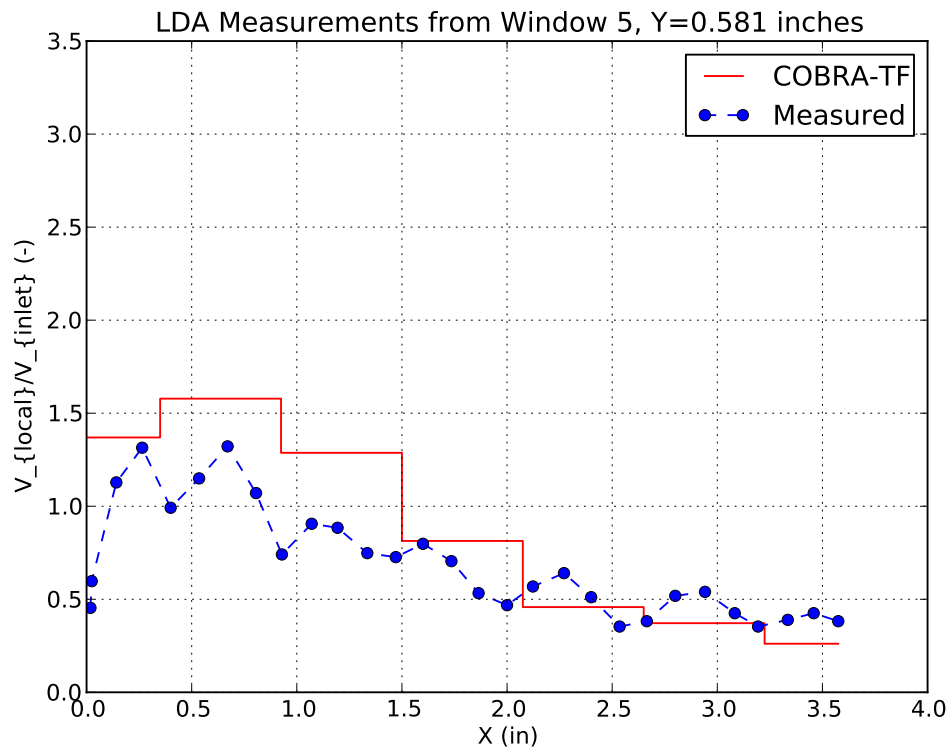

Figure 11.10: Predicted and measured subchannel velocities for Window 5 at Rake Location $\mathrm{Y}=0.581$ in in PNNL 2x6 


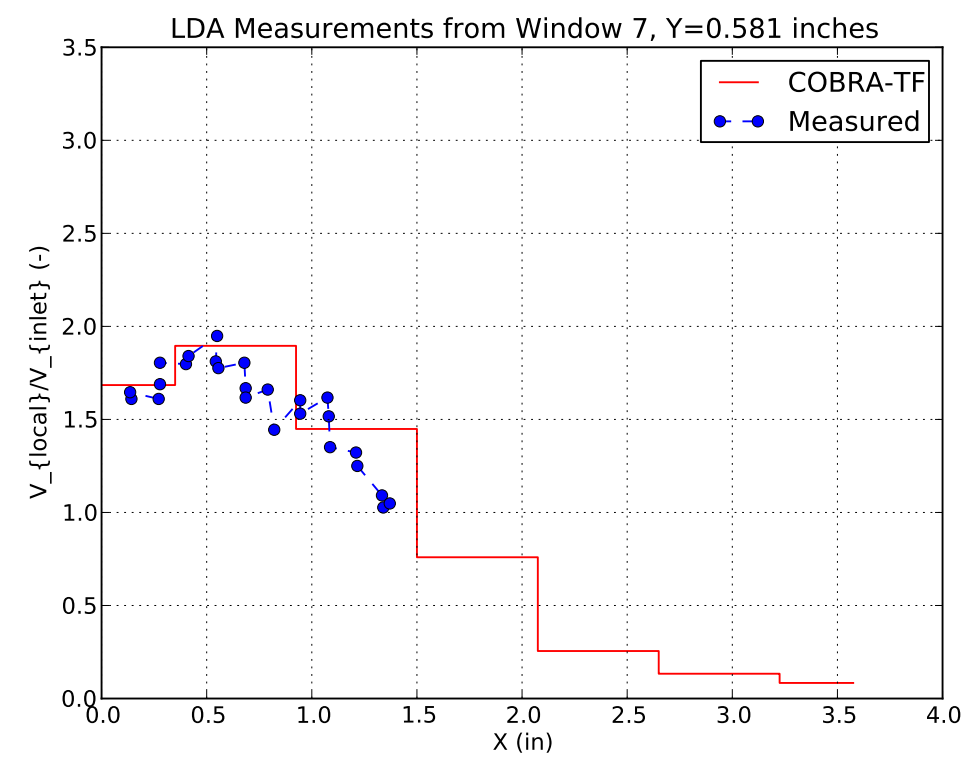

Figure 11.11: Predicted and measured subchannel velocities for Window 7 at Rake Location $\mathrm{Y}=0.581$ in in PNNL 2x6

in temperature is also unexpected. The predicted results are the behavior we would expect; temperatures should rise all the way up to the left side of the bundle. The authors noted this anomalous behavior, but did not provide a reason for its why it occurred. 


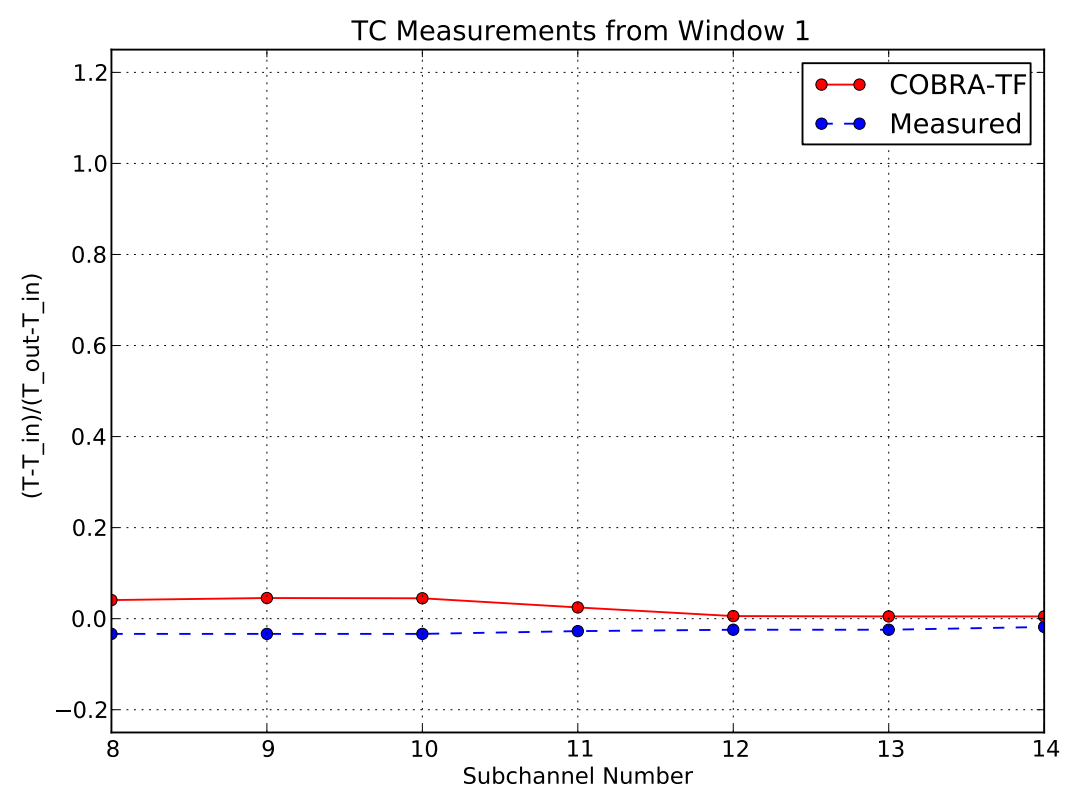

Figure 11.12: Predicted and measured subchannel-center temperatures for Window 1 in PNNL 2x6

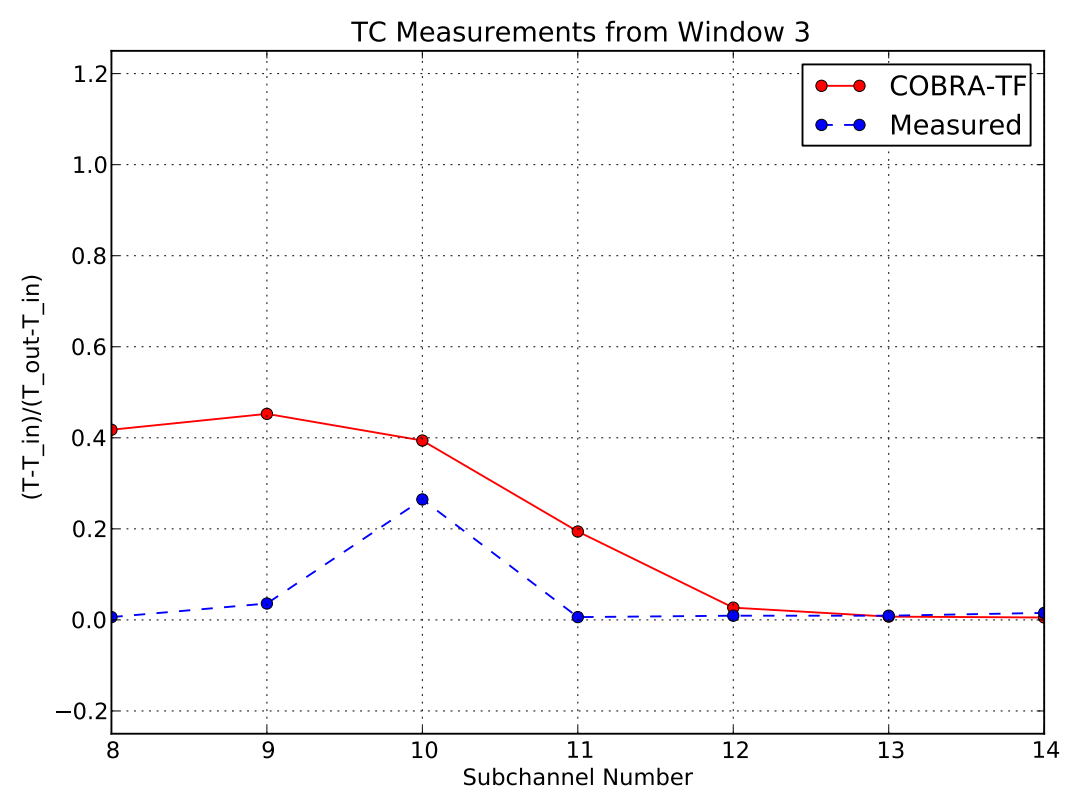

Figure 11.13: Predicted and measured subchannel-center temperatures for Window 3 in PNNL 2x6 


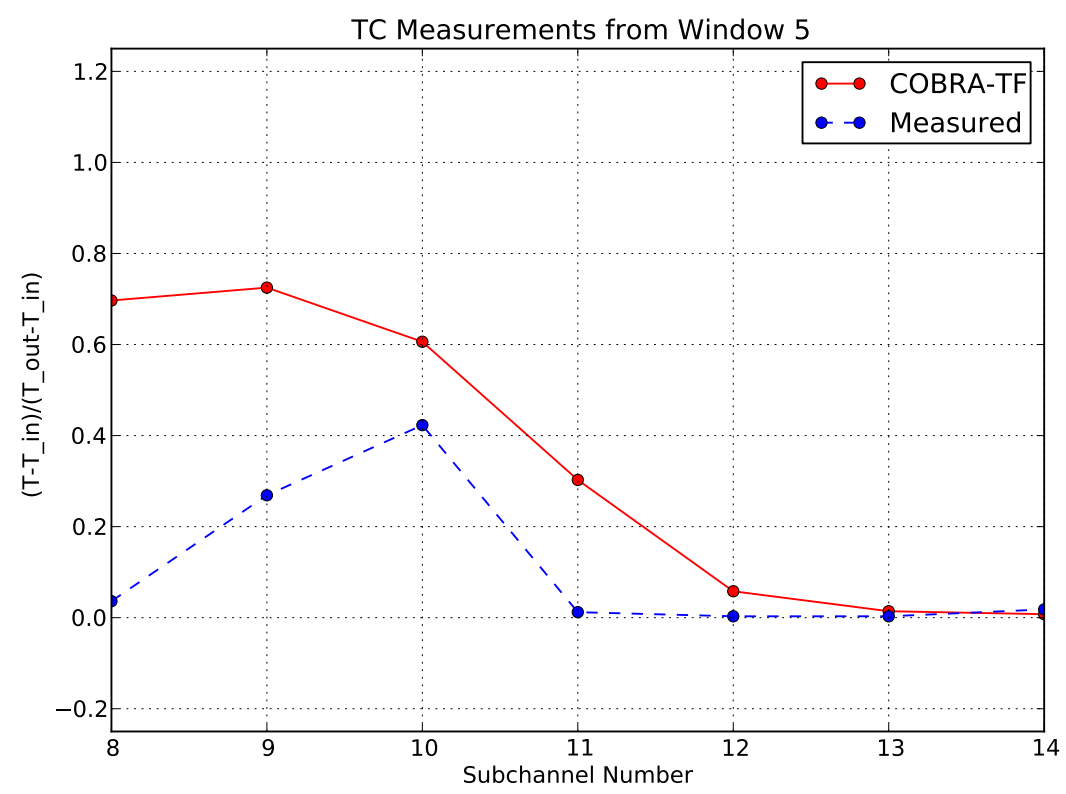

Figure 11.14: Predicted and measured subchannel-center temperatures for Window 5 in PNNL 2x6

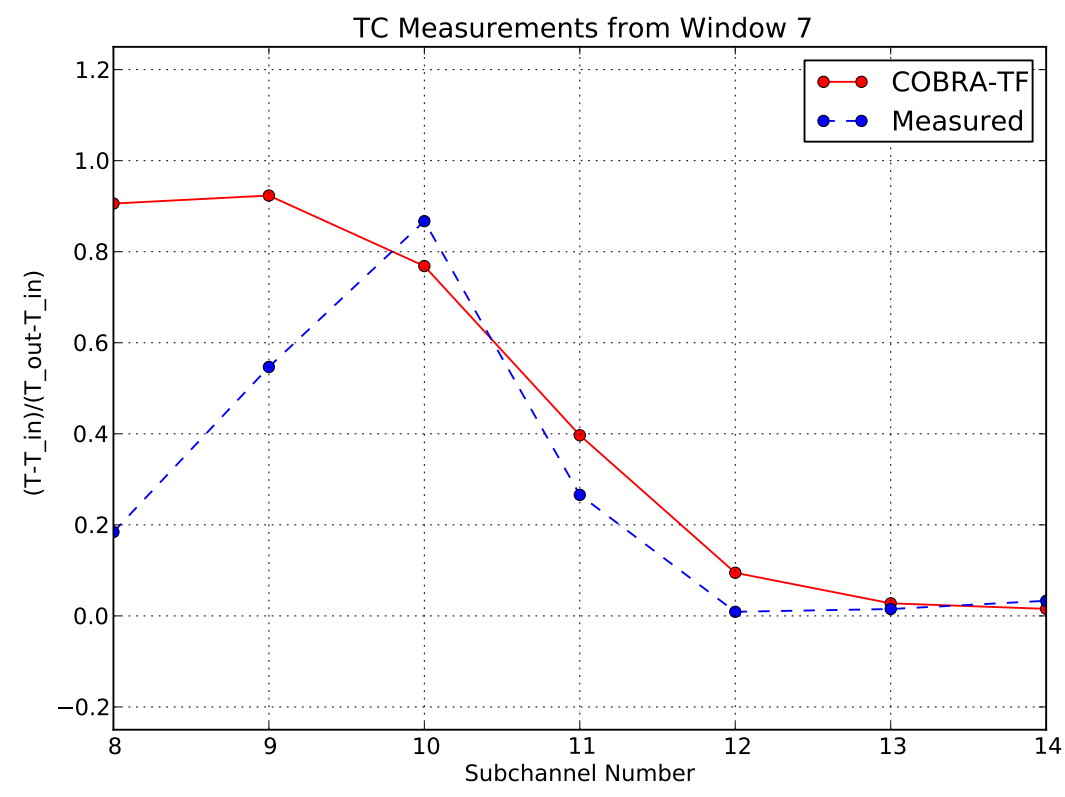

Figure 11.15: Predicted and measured subchannel-center temperatures for Window 7 in PNNL 2x6 


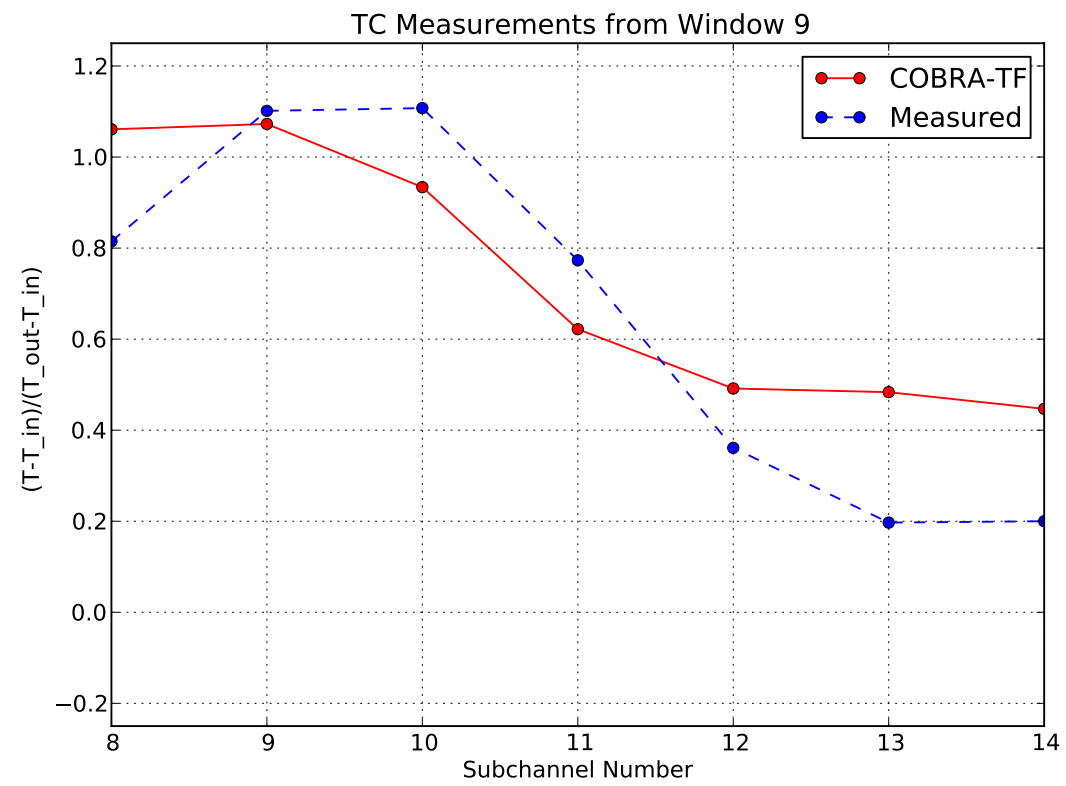

Figure 11.16: Predicted and measured subchannel-center temperatures for Window 9 in PNNL $2 \mathrm{x} 6$ 


\section{CHAPTER 12}

CONCLUSION

This document has been created as a means to validate CTF. It outlines the code requirements (capabilities) and then demonstrates the code's ability to to meet a significant portion of those capabilities. A variety of tests have been chosen to target the most important features of CTF, including pressure drop, void, rod and coolant temperature, and mixing prediction in rod bundle geometries. Qualitative and quantitative analysis was performed to assess CTFs ability to capture the trends and behavior of phenomena as well as to present a general measure of its accuracy.

There are some noted shortfalls in the CTF testing and documentation matrix. First, the validation studies performed and included in this document are not an exhaustive measure of the CTF capabilities; there are still more CTF features, noted in Chapter 2, that have yet to be validated. Second, the experimental data employed to validate CTF does not specifically single out individual physical models in CTF; rather, they exercise several models in combination.

This document does take great strides in improving CTF testing and validation. The tests modeled in this document act to exercise the most important code features of CTF. So long as the user is utilizing the code to model similar tests, utilizing the exercised features in this document, they can now have a greater confidence that CTF is actually capable of producing meaningful results. Furthermore, this document is meant to be a living document, giving the developers a forum for discussing future validation tasks and exercising different CTF capabilities. 
BIBLIOGRAPHY

[1] M.N. Avramova. CTF: A Thermal Hydraulic Sub-Channel Code for LWR Transient Analyses, User's Manual. Feb. 2009.

[2] R.K. Salko and M.N. Avramova. CTF Theory Manual. The Pennsylvania State University.

[3] A. Rubin et al. OECD/NRC BENCHMARK BASED ON NUPEC PWR SUBCHANNEL AND BUNDLE TESTS (PSBT). Tech. rep. US NRC and OECD Nuclear Energy Agency, 2010.

[4] B. Neykov et al. NUPEC BWR Full-size Fine-mesh Bundle Test (BFBT) Benchmark. Tech. rep. NUCLEAR ENERGY AGENCY, 2006.

[5] B.S. Shiralkar and D.W. Radcliffe. An experimental and analytical study of the synthesis of grid spacer loss coefficients. Tech. rep. NEDE-13181. General Electric, 1971.

[6] M. Avramova, K. Ivanov, and L. Hochreiter. "Analysis of Steady State and Transient Void Distribution Predictions for Phase I of the OECD/NEA BFBT Benchmark using CTF/NEM". In: The 12th International Topical Meeting on Nuclear Reactor Thermal Hydraulics (NURETH-12). 2007.

[7] M.S. Quigley, C.A. McMonagle, and J.M. Bates. Investigation of Combined Free and Forced Convection in a $2 x 6$ Rod Bundle. Tech. rep. BNWL-2216. Battelle Pacific Northwest Laboratories, 1997.

[8] J.M. Bates and E.U. Khan. Investigation of Combined Free and Forced Convection in a $2 x 6$ Rod Bundle during Controlled Flow Transients. Tech. rep. PNL-3135. Battelle Pacific Northwest laboratories, 1980.

[9] Z. Karoutas et al. Subcooled Boiling Data from Rod Bundles. Tech. rep. 1003383. EPRI, 2002.

[10] R.T. Lahey and F.J. Moody. The Thermal Hydraulics of a Boiling Water Nuclear Reactor, Second Edition. American Nuclear Society, 1993.

[11] D. W. Radcliffe R. T. Lahey Jr. B. S. Shirlakar. Two-Phase Flow and Heat Transfer In Multirod Geometries: Subchannel and Pressure Drop Measurements in a Nine-Rod Bundle for Diabatic and Adiabatic Conditions. Tech. rep. General Electric, 1970. 
[12] A.W. Bennett et al. Heat transfer to steam-water mixtures flowing in uniformly heated tubes in which the critical heat flux has been exceeded. Tech. rep. AERE-R 5373. U.K.A.E.A. Research Group, Atomic Energy Research Establishement, 1967.

[13] O. Nylund, K.M. Briker, R. Eklund, et al. FRIGG Loop Project. Tech. rep. R4-447/RTL1007. AB Atomenergi, Stockholm, 1968.

[14] R.W. Sterner and R.T. Lahey. Air/Water Subchannel Measurements of the Equilibrium Quality and Mass Flux Distribution in a Rod Bundle. Tech. rep. Rensselaer Polytechnic Institute, 1983.

[15] M. Sadatomi et al. "Single- and Two-Phase Turbulent Mixing Rate between Adjacent Subchanels in a Vertical $2 \times 3$ Rod Array Channel". In: International Journal of Multiphase Flow 30 (2004), pp. 481-498.

[16] M. Sadatomi et al. "Flow Characteristics in Hydraulically Equilibrium Two-Phase Flows in a Vertical $2 \times 3$ Rod Bundle Channel". In: International Journal of Multiphase Flow 30 (2004), pp. 1093-1119.

[17] M. Sadatomi, Y. Sato, and S. Saruwatari. "Two-Phase Flow in Vertical Noncircular Channels". In: International Journal of Multiphase Flow 8 (1982), pp. 641-645.

[18] Y. Sung et al. Application of Multi-Scale Thermal-Hydraulic Models to DNB Analysis. Tech. rep. CASL-I-2014-0119-000. Oak Ridge National Laboratory, 2014.

[19] Neil E. Todreas and Mujid S. Kazimi. Nuclear Systems II: Elements of Thermal Hydraulic Design. Hemisphere Publishing Corporation, 1990.

[20] B. Neykov, M. Avramova, and K. Ivanov. "Phase I-Void Distribution Final Results". In: $6^{\text {th }}$ OECD/NRC BFBT Workshop. 2009.

[21] R.W. Bowring. A Simple but Accurate Round Tube, Uniform Heat Flux Dryout Correlation over the Pressure Range 0.7 to 17 MPa. Tech. rep. AEE Winfrith, 1972.

[22] Neil E. Todreas and Mujid S. Kazimi. Nuclear Systems I: Thermal Hydraulic Fundamentals, $2^{\text {nd }}$ Edition. CRC press, 2011.

[23] D.C. Groeneveld et al. The 2006 CHF look-up table. Tech. rep. Nuclear Engineering and Design, 2007.

[24] D.C. Groeneveld et al. Look-Up Tables for Predicting CHF and Film Boiling Heat Transfer - Past, Present and Future (NURETH). Tech. rep. Chalk River Lab, AECL, 2003. 


\section{Acronyms}

BFBT BWR Full-size Fine-mesh Bundle Tests

BOHL Beginning of Heated Length

BWR Boiling Water Reactor

CASL Consortium for Advanced Simulation of Light Water Reactors

CIPS Crud-induced power shift

COBRA-TF Coolant-Boiling in Rod Arrays- Two Fluids

CTF PSU RDFMG version of COBRA-TF

CHF Critical Heat Flux

DNB Departure from Nucleate Boiling

GE General Electric

HTC Heat Transfer Coefficient

JAERI Japanese Atomic Energy Research Institute

LDA Laser Doppler Anemometer

LWR Light Water Reactor

MV Mixing Vane

MVG Mixing Vane Grid 
NMV Non-Mixing Vane

PSBT PWR Sub-channel and Bundle Tests

PSU Pennsylvania State University

PWR Pressurized Water Reactor

QOI Quantity of Interest

RDFMG Reactor Dynamics and Fuel Management Group

RIA Reactivity-Insertion Accident

rRMS relative root-mean-square

RMSE Root-mean-square error

$\mathbf{T} / \mathbf{H}$ Thermal/Hydraulic

V\&V Validation \& Verification 